\title{
MASCOT Search Results
}

\section{Protein View: TP84_03}

\author{
Database: TP84 \\ Score: 261 \\ Nominal mass $\left(\mathbf{M}_{\mathbf{r}}\right): 26292$ \\ Calculated pI: $\quad 9.17$
}

Sequence similarity is available as an NCBI BLAST search of 58. against $\mathbf{n r}$.

\section{Search parameters}

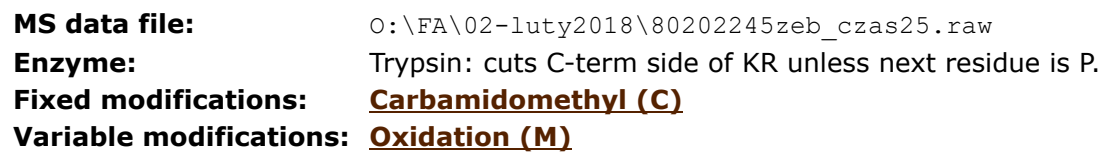

\section{Protein sequence coverage: $22 \%$}

Matched peptides shown in bold red.

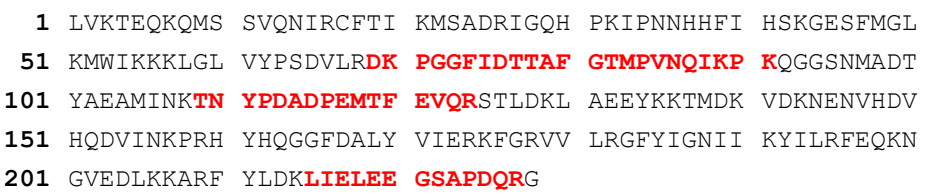

Unformatted sequence string: $\underline{\mathbf{2 2 8}}$ residues (for pasting into other applications).

Sort peptides by $\odot$ Residue Number $\bigcirc$ Increasing Mass $\bigcirc$ Decreasing Mass

Show predicted peptides also

\begin{tabular}{|c|c|c|c|c|c|c|c|c|c|c|c|}
\hline Query & Start - End & Observed & $\operatorname{Mr}(\exp t)$ & $\operatorname{Mr}(\mathrm{calc})$ & ppm & $\mathbf{M}$ & Score & Expect & Rank & $\mathrm{U}$ & Peptide \\
\hline $\mathbb{J}^{33512}$ & $69-91$ & 821.4311 & 2461.2714 & 2461.2675 & 1.56 & $\ddot{0}$ & 92 & $6.3 e-10$ & 1 & $\mathrm{U}$ & R.DKPGGFIDTTAFGTMPVNQIKPK . Q \\
\hline$\triangle 026656$ & $109-124$ & 956.9213 & 1911.8281 & 1911.8309 & -1.43 & 0 & 141 & $8.6 e-15$ & 1 & $\mathrm{U}$ & K. TNYPDADPEMTFEVQR.S \\
\hline$\nabla^{\prime} 16446$ & $215-227$ & 728.8669 & 1455.7192 & 1455.7205 & -0.90 & 0 & 54 & $4.1 e-06$ & 1 & $\mathrm{U}$ & K. LIELEEGSAPDQR. G \\
\hline
\end{tabular}

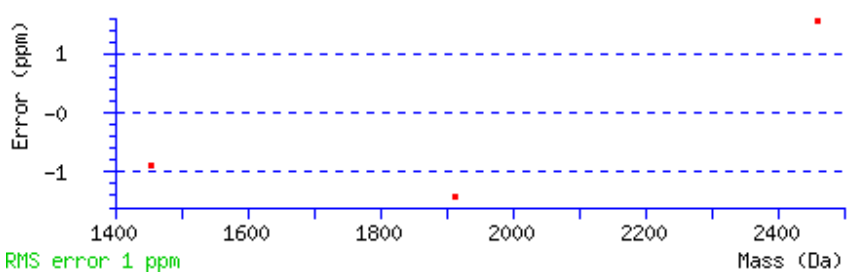




\section{MASCOT Search Results}

\section{Protein View: TP84_03}

\author{
Database: \\ TP84 \\ Score: \\ Nominal mass $\left(M_{\mathbf{r}}\right): 26292$ \\ Calculated pI: $\quad 9.17$
}

Sequence similarity is available as an NCBI BLAST search of 58. against nr.

\section{Search parameters}

\section{MS data file: \\ Enzyme: \\ 0: \FA \02-luty2018\80202246zeb czas30.raw \\ Fixed modifications: \\ Trypsin: cuts C-term side of KR unless next residue is $P$.}

\section{Protein sequence coverage: $\mathbf{2 8} \%$}

Matched peptides shown in bold red.

1 LVKTEQKQMS SVQNIRCFTI KMSADRIGQH PKIPNNHHFI HSKGESFMGL

51 KMWIKKKLGL VYPSDVLRDK PGGFIDTTAF GTMPVNOIKP KQGGSNMADT

101 YAEAMINKTN YPDADPEMTE EVQRSTLDKL AEEYKKTMDK VDKNENVHDV 151 HQDVINKPRH YHQGGFDALY VIERKFGRVV LRGFYIGNII KYILRFEQKN 201 GVEDLKKARF YLDKLIELEE GSAPDQRG

Unformatted sequence string: $\underline{\mathbf{2 2 8}}$ residues (for pasting into other applications).

Sort peptides by $\odot$ Residue Number $\bigcirc$ Increasing Mass $\bigcirc$ Decreasing Mass

\section{Show predicted peptides also}

\begin{tabular}{|c|c|}
\hline Query & Start - End \\
\hline 14652 & $58-68$ \\
\hline 14653 & $58-68$ \\
\hline 14654 & $58-68$ \\
\hline 14655 & $58-68$ \\
\hline$\sigma^{43216}$ & $69-91$ \\
\hline$\checkmark 43217$ & $69-91$ \\
\hline$\checkmark \underline{41117}$ & $141-159$ \\
\hline$\nearrow^{41119}$ & $141-159$ \\
\hline 35694 & $144-159$ \\
\hline 23071 & $215-227$ \\
\hline$\underline{23072}$ & $215-227$ \\
\hline 23073 & $215-227$ \\
\hline
\end{tabular}

$\begin{array}{ccr}\text { ppm } & \text { M } & \text { Score } \\ 0.23 & 0 & 57 \\ 0.36 & 0 & 56 \\ 0.98 & 0 & 54 \\ 1.60 & 0 & 62 \\ 1.49 & 0 & 71 \\ 1.99 & 0 & 51 \\ -1.45 & 1 & 102 \\ 0.075 & 1 & 71 \\ -0.011 & 0 & 77 \\ -0.47 & 0 & 90 \\ -0.25 & 0 & 90 \\ -0.080 & 0 & 104\end{array}$

Expect Rank U Peptide

$2 e-06 \quad 1 \quad \bar{U}$ K. LGLVYPSDVLR.D

2. $3 e-06 \quad 1$ U K. LGLVYPSDVLR. D

$4 e-06 \quad 1$ U K. LGLVYPSDVLR.D

$6.3 e-07 \quad 1 \quad$ U K. LGLVYPSDVLR. D

$8.2 e-08 \quad 1 \quad$ U R.DKPGGFIDTTAFGTMPVNQIKPK.Q

$8.3 e-06 \quad 1 \quad$ U R.DKPGGFIDTTAFGTMPVNQIKPK.Q

$6.2 e-11 \quad 1 \quad$ U $K$. VDKNENVHDVHODVINKPR.H

$7.2 e-08$ I U K. VDKNENVHDVHQDVINKPR.H

$2 e-08 \quad 1 \quad$ U K. NENVHDVHODVINKPR.H

$9.6 e-10 \quad 1$ U K. LIELEEGSAPDQR.G

9. $5 e-10 \quad 1$ U K. LIELEEGSAPDOR.G

$3.9 e-11$ U U K. LIELEEGSAPDQR.G

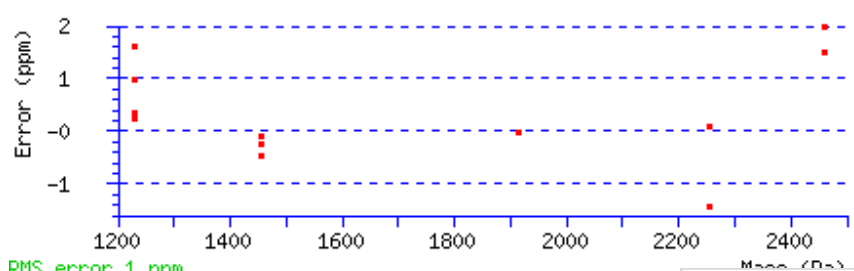

TP84_03

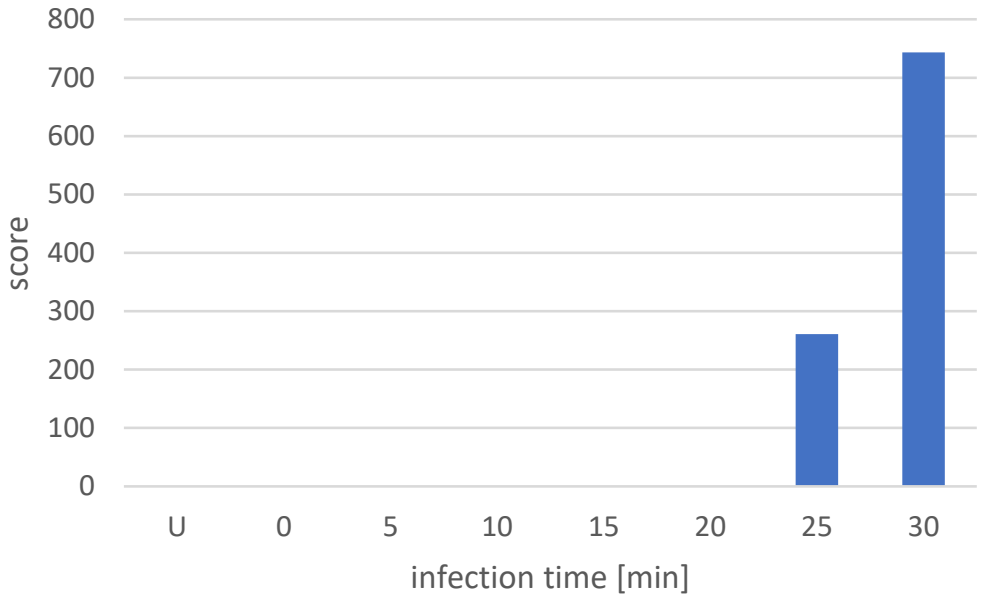




\title{
MASCOT Search Results
}

\section{Protein View: TP84_06}

\author{
Database: TP84 \\ Score: 194 \\ Nominal mass $\left(M_{\mathbf{r}}\right): 62259$ \\ Calculated pI: $\quad 4.96$
}

Sequence similarity is available as an NCBI BLAST search of 61. against nr.

\section{Search parameters}

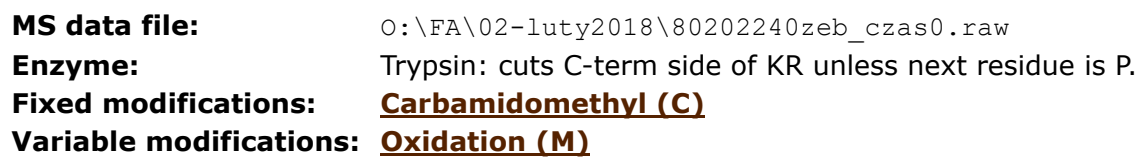

Protein sequence coverage: $5 \%$

Matched peptides shown in bold red.
1 MGIKWTKWST NVIKKYHGNI QKYRKLYDGD HAKLFERAKR LIQEGEITDQ
51 IIEGAEVARN VKTPYIVANV CKMIVDIPAM LVSRAIGQVT TSMSPDDFAG
101 MVTDQGDGTV ISLYEKQKEL IKGIAKRSNL QFEHKTNIIH HQMDGGIVGM
151 PFDDENGLRI EFKSRDVYYP HPDGRGCDLV YQLEIEDEET EEAIKYLHVY
201 RERVEEQKLV TQHMLYKIGE SGMLEEIEDE AEVKEILGIE KTYREFEGRD
251 KPFVVYWPNN KTETHPLGRS ELYNLAGKQD EINWTLTRNA IVYERNGKPR
301 IAVSKEIFQA LQDKAFERYG DENKIDHRDL EIVTFDENGK AMEVIQIDVS
351 KIGDIKWVKD LMKLMLMETH TSEKAVDFYL EGNTSAQSGI AKFYDLFVSI
401 MKAEQIATEY VHFLQELFEN CLWIAHQDDP DIVIEEPRIQ IKDMIPISRR
451 ELIEQESTAY KNGTQSLETT VRNQNPTATE DWIEDELAAI EESQQSTDTT
501 SILMGRQTLS NLLDNRNPNG TPIGAAQQQP QQGTPQTGGG QA

Unformatted sequence string: $\underline{\mathbf{5 4 2}}$ residues (for pasting into other applications).

Sort peptides by $\odot$ Residue Number $\bigcirc$ Increasing Mass $\bigcirc$ Decreasing Mass

Show predicted peptides also

$\begin{array}{rcrrr}\text { Query } & \text { Start }- \text { End } & \text { Observed } & \text { Mr (expt) } & \text { Mr (calc) } \\ \varangle 26286 & 41-59 & 1042.5502 & 2083.0858 & 2083.0797 \\ \varangle \underline{10714} & 451-461 & 655.8302 & 1309.6459 & 1309.6401\end{array}$

ppm M Score Expect Rank U Peptide

$\begin{array}{lllllll}2.96 & 0 & 139 & 1.2 e-14 & 1 & \text { U } & \text { R.LIQEGEITDOIIEGAEVAR.N }\end{array}$

$\widetilde{10714} \quad 451-461$

$655.8302 \quad 1309.6459 \quad 1309.6401$

$4.420 \quad 67$

$1.8 e-07 \quad \frac{1}{1}$

U R.ELIEQESTAYK.N

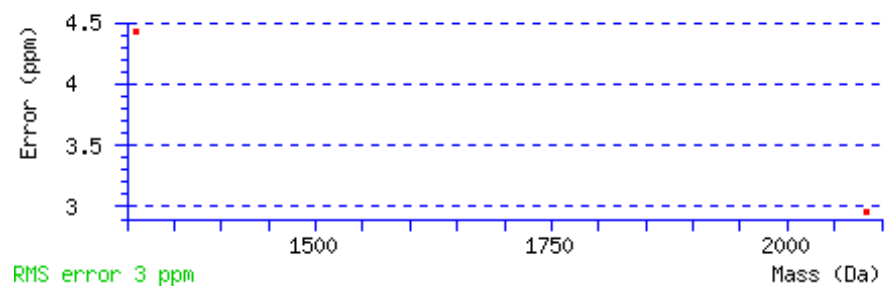




\section{MASCOT Search Results}

\section{Protein View: TP84_06}

$\begin{array}{ll}\text { Database: } & \text { TP84 } \\ \text { Score: } & 203 \\ \text { Nominal mass (Mr): } & 62259 \\ \text { Calculated pI: } & 4.96\end{array}$

Sequence similarity is available as an NCBI BLAST search of 61. against nr.

\section{Search parameters}

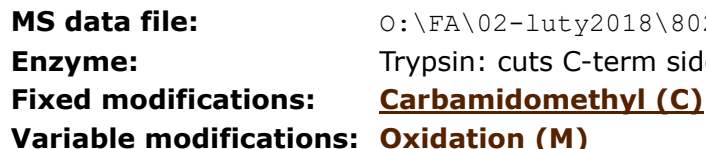

\section{Protein sequence coverage: $9 \%$}

Matched peptides shown in bold red.
1 MGIKWTKWST NVIKKYHGNI QKYRKLYDGD HAKLFERAKR LIQEGEITDQ
51 IIEGAEVARN VKTPYIVANV CKMIVDIPAM LVSRAIGQVT TSMSPDDFAG
101 MVTDQGDGTV ISLYEKQKEL IKGIAKRSNL QFEHKTNIIH HQMDGGIVGM
151 PFDDENGLRI EFKSRDVYYP HPDGRGCDLV YQLEIEDEET EEAIKYLHVY
201 RERVEEQKLV TQHMLYKIGE SGMLEEIEDE AEVKEILGIE KTYREFEGRD
251 KPFVVYWPNN KTFTHPLGRS ELYNLAGKQD EINWTLTRNA IVYERNGKPR
301 IAVSKEIFQA LQDKAFERYG DENKIDHRDL EIVTFDENGK AMEVIQIDVS
351 KIGDIKWVKD LMKLMLMETH TSEKAVDFYL EGNTSAQSGI AKFYDLFVSI
401 MKAEQIATEY VHFLQELFEN CLWIAHQDDP DIVIEEPRIQ IKDMIPISRR
451 ELIEQESTAY KNGTQSLETT VRNQNPTATE DWIEDELAAI EESQQSTDTT
501 SILMGRQTLS NLLDNRNPNG TPIGAAQQQP QQGTPQTGGG QA

Unformatted sequence string: $\mathbf{5 4 2}$ residues (for pasting into other applications).

Sort peptides by $\bullet$ Residue Number $\bigcirc$ Increasing Mass $\bigcirc$ Decreasing Mass

Show predicted peptides also

\begin{tabular}{|c|c|c|c|c|c|c|c|c|c|c|c|}
\hline Query & Start - End & Observed & $\operatorname{Mr}(\exp t)$ & $\operatorname{Mr}(\mathrm{calc})$ & ppm & $\mathbf{M}$ & Score & Expect & Rank & $\mathrm{U}$ & Peptide \\
\hline$\nabla 26156$ & $41-59$ & 1042.5348 & 2083.0550 & 2083.0797 & -11.8 & $\dddot{0}$ & 112 & $6.3 e-12$ & 1 & $\ddot{\mathrm{U}}$ & R. LIQEGEITDQIIEGAEVAR.N \\
\hline$\sigma^{\prime 1} 1659$ & $73-84$ & 672.8733 & 1343.7321 & 1343.7305 & 1.23 & 0 & 84 & $4.2 e-09$ & 1 & $\mathrm{U}$ & K.MIVDIPAMLVSR. A \\
\hline$\overline{8266}$ & $462-472$ & 603.3020 & 1204.5894 & 1204.6048 & -12.8 & 0 & 14 & 0.037 & 1 & $\mathrm{U}$ & K.NGTQSLETTVR.N \\
\hline$\overline{7528}$ & $507-516$ & 587.3221 & 1172.6297 & 1172.6149 & 12.6 & 0 & 32 & 0.0007 & 1 & U & R.QTLSNLLDNR.N \\
\hline
\end{tabular}

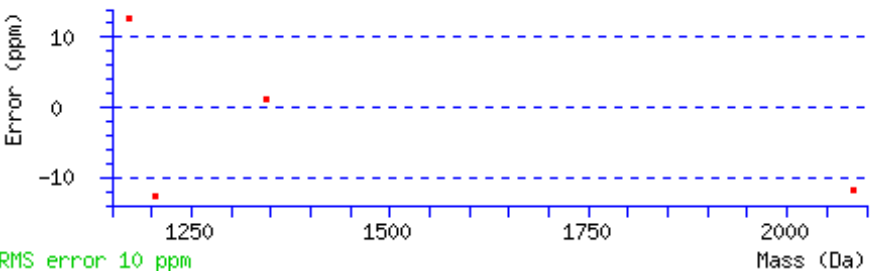




\section{MASCOT Search Results}

\section{Protein View: TP84_06}

$\begin{array}{ll}\text { Database: } & \text { TP84 } \\ \text { Score: } & 148 \\ \text { Nominal mass (Mr): } & 62259 \\ \text { Calculated pI: } & 4.96\end{array}$

Sequence similarity is available as an NCBI BLAST search of 61. against nr.

\section{Search parameters}

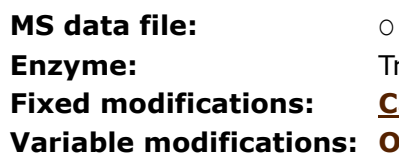

\section{Protein sequence coverage: $9 \%$}

Matched peptides shown in bold red.
1 MGIKWTKWST NVIKKYHGNI QKYRKLYDGD HAKLFERAKR LIQEGEITDQ
51 IIEGAEVARN VKTPYIVANV CKMIVDIPAM LVSRAIGQVT TSMSPDDFAG 101 MVTDQGDGTV ISLYEKQKEL IKGIAKRSNL QFEHKTNIIH HQMDGGIVGM 151 PFDDENGLRI EFKSRDVYYP HPDGRGCDLV YQLEIEDEET EEAIKYLHVY 201 RERVEEQKLV TQHMLYKIGE SGMLEEIEDE AEVKEILGIE KTYREFEGRD 251 KPFVVYWPNN KTFTHPLGRS ELYNLAGKQD EINWTLTRNA IVYERNGKPR 301 IAVSKEIFQA LQDKAFERYG DENKIDHRDL EIVTFDENGK AMEVIQIDVS 351 KIGDIKWVKD LMKLMLMETH TSEKAVDFYL EGNTSAQSGI AKFYDLFVSI 401 MKAEQIATEY VHFLQELFEN CLWIAHQDDP DIVIEEPRIQ IKDMIPISRR 451 ELIEQESTAY KNGTQSLETT VRNQNPTATE DWIEDELAAI EESQQSTDTT 501 SILMGRQTLS NLLDNRNPNG TPIGAAQQQP QQGTPQTGGG QA

Unformatted sequence string: $\underline{\mathbf{5 4 2}}$ residues (for pasting into other applications).

Sort peptides by $\odot$ Residue Number $\bigcirc$ Increasing Mass $\bigcirc$ Decreasing Mass

Show predicted peptides also

\begin{tabular}{|c|c|}
\hline Query & Start - End \\
\hline$\nabla^{\prime 24737}$ & $41-59$ \\
\hline$\overline{10426}$ & $451-461$ \\
\hline$\checkmark 7951$ & $462-472$ \\
\hline$\overline{7952}$ & $462-472$ \\
\hline$\overline{7954}$ & $462-472$ \\
\hline Ø7286 & $507-516$ \\
\hline
\end{tabular}

$\begin{array}{ccc}\text { Observed } & \text { Mr (expt) } & \text { Mr (calc) } \\ 695.3661 & 2083.0766 & 2083.0797 \\ 655.8313 & 1309.6481 & 1309.6401 \\ 603.3011 & 1204.5876 & 1204.6048 \\ 603.3012 & 1204.5878 & 1204.6048 \\ 603.3028 & 1204.5910 & 1204.6048 \\ 587.3177 & 1172.6208 & 1172.6149\end{array}$

\begin{tabular}{rrr}
\multicolumn{1}{c}{ ppm } & M & Score \\
-1.50 & 0 & 82 \\
6.11 & 0 & 64 \\
-14.3 & 0 & 15 \\
-14.1 & 0 & 13 \\
-11.4 & 0 & 15 \\
5.03 & 0 & 25
\end{tabular}

Expect Rank U Peptide

6.8e-09 1 U R.LIQEGEITDQIIEGAEVAR.N

3.8e-07 1 U R.ELIEQESTAYK.N

$0.035 \quad 1$ U K.NGTQSLETTVR.N

$0.049 \quad 1$ U K.NGTQSLETTVR.N

$0.034 \quad 1$ U K.NGTQSLETTVR.N

$\checkmark \underline{7286} \quad 507-516$

0.034

$\begin{array}{ll}\text { U } & \text { K.NGTQSLETTVR.N } \\ \text { U } & \text { R. QTLSNLLDNR.N }\end{array}$

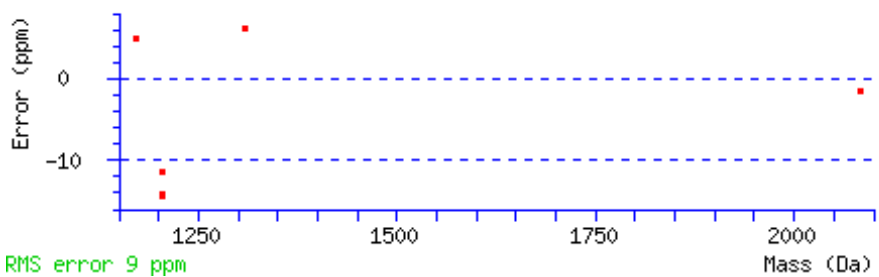




\title{
MASCOT Search Results
}

\section{Protein View: TP84_06}

$\begin{array}{ll}\text { Database: } & \text { TP84 } \\ \text { Score: } & 393 \\ \text { Nominal mass (Mr): } & 62259 \\ \text { Calculated pI: } & 4.96\end{array}$

Sequence similarity is available as an NCBI BLAST search of 61. against nr.

\section{Search parameters}

\author{
MS data file: \\ O: \FA $\backslash 02$-luty2018\80202245zeb czas25.raw \\ Enzyme: \\ Trypsin: cuts C-term side of $K R$ unless next residue is $P$. \\ Fixed modifications: \\ Carbamidomethyl (C) \\ Variable modifications: Oxidation (M)
}

\section{Protein sequence coverage: $9 \%$}

Matched peptides shown in bold red.
1 MGIKWTKWST NVIKKYHGNI QKYRKLYDGD HAKLFERAKR LIQEGEITDQ
51 IIEGAEVARN VKTPYIVANV CKMIVDIPAM LVSRAIGQVT TSMSPDDFAG
101 MVTDQGDGTV ISLYEKQKEL IKGIAKRSNL QFEHKTNIIH HQMDGGIVGM
151 PFDDENGLRI EFKSRDVYYP HPDGRGCDLV YQLEIEDEET EEAIKYLHVY
201 RERVEEQKLV TQHMLYKIGE SGMLEEIEDE AEVKEILGIE KTYREFEGRD
251 KPFVVYWPNN KTFTHPLGRS ELYNLAGKQD EINWTLTRNA IVYERNGKPR
301 IAVSKEIFQA LQDKAFERYG DENKIDHRDL EIVTFDENGK AMEVIQIDVS
351 KIGDIKWVKD LMKLMLMETH TSEKAVDFYL EGNTSAQSGI AKFYDLFVSI
401 MKAEQIATEY VHFLQELFEN CLWIAHQDDP DIVIEEPRIQ IKDMIPISRR
451 ELIEQESTAY KNGTQSLETT VRNQNPTATE DWIEDELAAI EESQQSTDTT
501 SILMGRQTLS NLLDNRNPNG TPIGAAQQQP QQGTPQTGGG QA

Unformatted sequence string: $\underline{\mathbf{5 4 2}}$ residues (for pasting into other applications).

Sort peptides by $\odot$ Residue Number $\bigcirc$ Increasing Mass $\bigcirc$ Decreasing Mass

Show predicted peptides also

\begin{tabular}{|c|c|c|c|c|c|c|c|c|c|c|c|}
\hline Query & Start - End & Observed & $M r(\exp t)$ & $\operatorname{Mr}(\mathrm{calc})$ & ppm & M & Score & Expect & Rank & $\mathrm{U}$ & Peptide \\
\hline$\square \underline{29292}$ & $41-59$ & 1042.5436 & 2083.0726 & 2083.0797 & -3.38 & $\dddot{0}$ & 164 & $4.3 e-17$ & 1 & $\ddot{\mathrm{U}}$ & R. LIQEGEITDQIIEGAEVAR.N \\
\hline$\checkmark \underline{29295}$ & $41-59$ & 695.3705 & 2083.0896 & 2083.0797 & 4.75 & 0 & 51 & $8.1 e-06$ & 1 & $\mathrm{U}$ & R. LIQEGEITDQI IEGAEVAR . N \\
\hline 13606 & $73-84$ & 672.8727 & 1343.7309 & 1343.7305 & 0.33 & 0 & 93 & $4.6 e-10$ & 1 & $\mathrm{U}$ & K.MIVDIPAMLVSR.A \\
\hline$\checkmark 12923$ & $364-374$ & 440.5489 & 1318.6248 & 1318.6261 & -1.01 & 0 & 59 & $1.2 e-06$ & 1 & $\mathrm{U}$ & K. LMLMETHTSEK. A \\
\hline$\underline{12675}$ & $451-461$ & 655.8292 & 1309.6439 & 1309.6401 & 2.88 & 0 & 78 & $1.4 e-08$ & 1 & $\mathrm{U}$ & R.ELIEQESTAYK.N \\
\hline
\end{tabular}

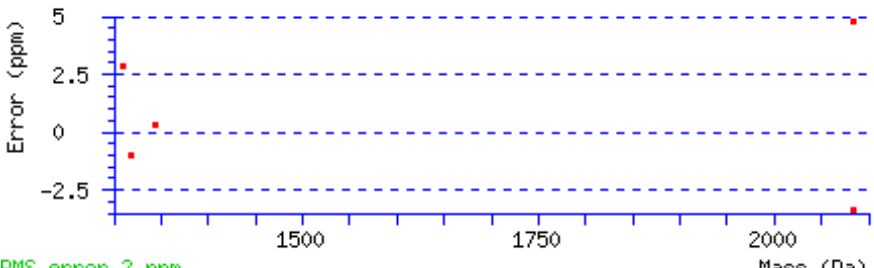




\section{MASCOT Search Results}

\section{Protein View: TP84_06}

$\begin{array}{ll}\text { Database: } & \text { TP84 } \\ \text { Score: } & 829 \\ \text { Nominal mass }\left(M_{\mathbf{r}}\right): & 62259 \\ \text { Calculated pI: } & 4.96\end{array}$

Sequence similarity is available as an NCBI BLAST search of 61. against nr.

\section{Search parameters}

$\begin{array}{ll}\text { MS data file: } & 0: \backslash F A \backslash 02-l u t y 2018 \backslash 80202246 z e b \_c z a s 30 . \text { raw } \\ \text { Enzyme: } & \text { Trypsin: cuts C-term side of KR unless next residue is P. } \\ \text { Fixed modifications: } & \text { Carbamidomethyl (C) } \\ \text { Variable modifications: } & \text { Oxidation }(\mathbf{M})\end{array}$

\section{Protein sequence coverage: $11 \%$}

Matched peptides shown in bold red.
1 MGIKWTKWST NVIKKYHGNI QKYRKLYDGD HAKLFERAKR LIQEGEITDQ
51 IIEGAEVARN VKTPYIVANV CKMIVDIPAM LVSRAIGQVT TSMSPDDFAG 101 MVTDQGDGTV ISLYEKQKEL IKGIAKRSNL QFEHKTNIIH HQMDGGIVGM 151 PFDDENGLRI EFKSRDVYYP HPDGRGCDLV YQLEIEDEET EEAIKYLHVY 201 RERVEEQKLV TQHMLYKIGE SGMLEEIEDE AEVKEILGIE KTYREFEGRD 251 KPFVVYWPNN KTFTHPLGRS ELYNLAGKQD EINWTLTRNA IVYERNGKPR 301 IAVSKEIFQA LQDKAFERYG DENKIDHRDL EIVTFDENGK AMEVIQIDVS 351 KIGDIKWVKD LMKLMLMETH TSEKAVDFYL EGNTSAQSGI AKFYDLFVSI 401 MKAEQIATEY VHFLQELFEN CLWIAHQDDP DIVIEEPRIQ IKDMIPISRR 451 ELIEQESTAY KNGTQSLETT VRNQNPTATE DWIEDELAAI EESQQSTDTT 501 SILMGRQTLS NLLDNRNPNG TPIGAAQQQP QQGTPQTGGG QA

Unformatted sequence string: $\underline{\mathbf{5 4 2}}$ residues (for pasting into other applications).

Sort peptides by $\odot$ Residue Number $\bigcirc$ Increasing Mass $\bigcirc$ Decreasing Mass

Show predicted peptides also

\begin{tabular}{|c|c|c|c|c|c|c|c|c|c|c|c|}
\hline Query & Start - End & Observed & Mr (expt) & $\mathrm{Mr}(\mathrm{calc})$ & ppm & $\mathbf{M}$ & Score & Expect & Rank & $\mathrm{U}$ & Peptide \\
\hline $\mathbb{J}^{38519}$ & $41-59$ & 1042.5436 & 2083.0726 & 2083.0797 & -3.38 & 0 & 136 & $2.4 e-14$ & 1 & $\dddot{\mathrm{U}}$ & R. LIQEGEITDQI IEGAEVAR.N \\
\hline$\sigma^{\prime 38520}$ & $41-59$ & 1042.5476 & 2083.0806 & 2083.0797 & 0.46 & 0 & 148 & $1.6 e-15$ & 1 & $\mathrm{U}$ & R. LIQEGEITDQI IEGAEVAR.N \\
\hline$\checkmark 38521$ & $41-59$ & 695.3682 & 2083.0827 & 2083.0797 & 1.47 & 0 & 68 & $1.7 e-07$ & 1 & $\mathrm{U}$ & R.LIQEGEITDQI IEGAEVAR.N \\
\hline $\mathbb{J}^{\prime 38522}$ & $41-59$ & 695.3686 & 2083.0839 & 2083.0797 & 2.02 & 0 & 67 & $2.1 e-07$ & 1 & $\mathrm{U}$ & R. LIQEGEITDQIIEGAEVAR.N \\
\hline$\checkmark 19164$ & $73-84$ & 672.8729 & 1343.7312 & 1343.7305 & 0.56 & 0 & 76 & $2.7 e-08$ & 1 & $\mathrm{U}$ & K.MIVDIPAMLVSR. A \\
\hline$\square^{\prime 19165}$ & $73-84$ & 672.8729 & 1343.7313 & 1343.7305 & 0.59 & 0 & 89 & $1.3 e-09$ & 1 & $\mathrm{U}$ & K.MIVDIPAMLVSR. A \\
\hline$\mho^{\prime 19166}$ & $73-84$ & 672.8732 & 1343.7319 & 1343.7305 & 1.08 & 0 & 87 & $2 e-09$ & 1 & $\mathrm{U}$ & K.MIVDIPAMLVSR. A \\
\hline$\square \underline{24704}$ & $250-261$ & 753.8862 & 1505.7579 & 1505.7667 & -5.83 & 0 & 70 & $8.9 e-08$ & 1 & $\mathrm{U}$ & R.DKPFVVYWPNNK. T \\
\hline$ð^{2} \underline{24705}$ & $250-261$ & 753.8913 & 1505.7681 & 1505.7667 & 0.94 & 0 & 78 & $1.4 e-08$ & 1 & $\mathrm{U}$ & R.DKPFVVYWPNNK. T \\
\hline$\mho^{\prime} 16512$ & $279-288$ & 638.3181 & 1274.6216 & 1274.6255 & -3.07 & 0 & 80 & $9.2 e-09$ & 1 & $\mathrm{U}$ & K. QDEINWTLTR.N \\
\hline$\checkmark 17805$ & $451-461$ & 655.8290 & 1309.6435 & 1309.6401 & 2.60 & 0 & 59 & $1.2 e-06$ & 1 & $\mathrm{U}$ & R.ELIEQESTAYK.N \\
\hline
\end{tabular}

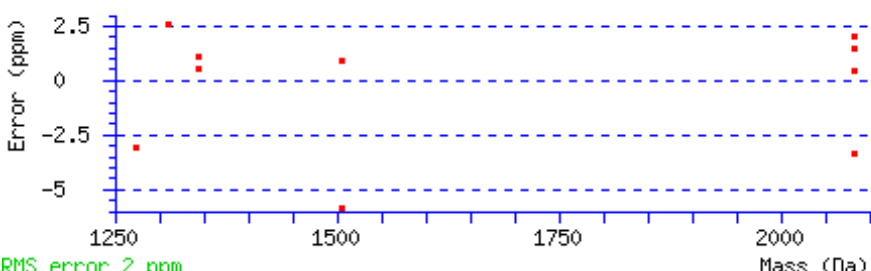


TP84_06

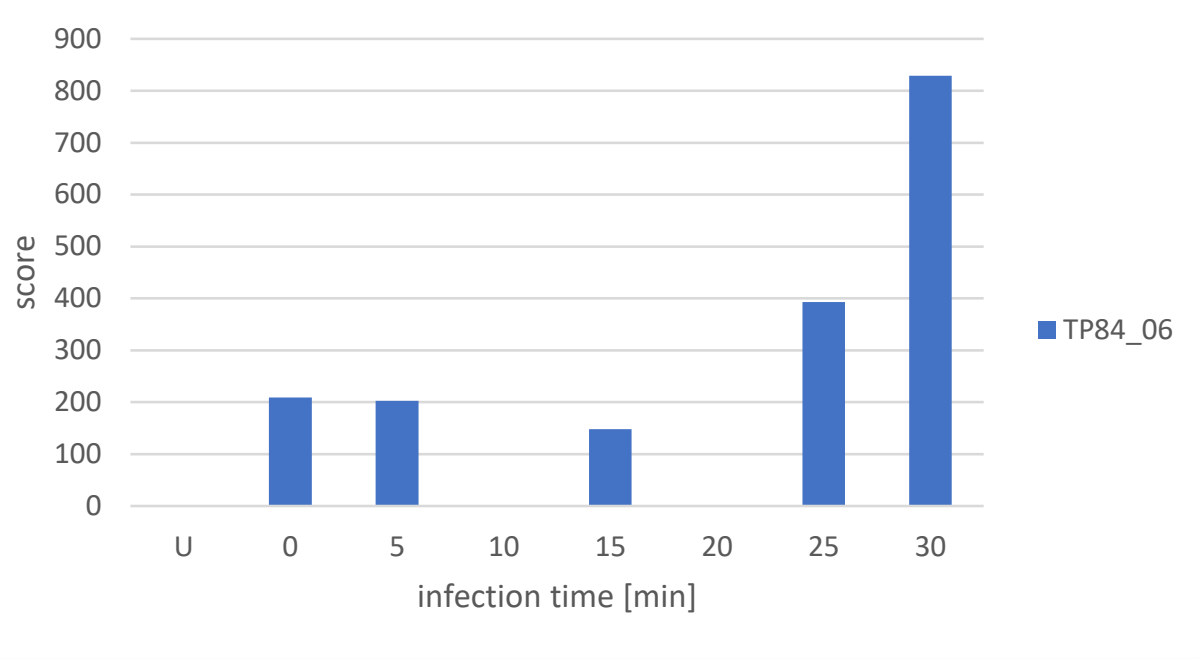




\title{
MASCOT Search Results
}

\section{Protein View: TP84_10}

\author{
Database: $\quad$ TP84 \\ Score: 387 \\ Nominal mass $\left(\mathbf{M}_{\mathbf{r}}\right): 31601$ \\ Calculated pI: $\quad 4.70$
}

Sequence similarity is available as an NCBI BLAST search of 65 . against nr.

\section{Search parameters}

\section{MS data file: \\ Enzyme: \\ Fixed modifications: \\ Variable modifications: Oxidation (M)}

\section{Protein sequence coverage: $15 \%$}

Matched peptides shown in bold red.

1 MQDKQITDEF IQKANKASEF GFLQFFAGEG GQQGGEPTDP TDPTDPLDDP

51 EGGEPNDPTD PTDPVDDQDD NDDDTGITYE KGLIDRILKA NNIDFGQLLK 101 DNPELKRQYQ ARFNKNMSKR LEKYQGVDVD EYFELKKRAE SGNLEGDAKT 151 WKEKYEQLKA EMETTTKKTA IQQYAIENGF DSEQIAFITS MIDMNKLERD 201 DEGEWMGIDE EVERIKEKFP RMFEPRDGQV GGTPKKESKY NPGTKKHNQD 251 TKPTDPRELG RQIALQRHKN RLKQQ

Unformatted sequence string: $\mathbf{2 7 5}$ residues (for pasting into other applications).

Sort peptides by $\bullet$ Residue Number $\bigcirc$ Increasing Mass $\bigcirc$ Decreasing Mass

Show predicted peptides also

\begin{tabular}{|c|c|}
\hline Query & Start - End \\
\hline$\sigma^{\prime 1} 10489$ & $90-100$ \\
\hline$\triangle 10490$ & $90-100$ \\
\hline$\nabla^{\prime} 10491$ & $90-100$ \\
\hline$\checkmark \underline{6847}$ & $139-149$ \\
\hline$-\underline{6848}$ & $139-149$ \\
\hline$\nearrow^{2} 2841$ & $160-167$ \\
\hline 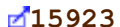 & $246-257$ \\
\hline
\end{tabular}

$\begin{array}{rrr}\text { Observed } & \text { Mr (expt) } & \text { Mr (calc) } \\ 616.8359 & 1231.6572 & 1231.6561 \\ 616.8361 & 1231.6576 & 1231.6561 \\ 616.8366 & 1231.6586 & 1231.6561 \\ 545.7547 & 1089.4948 & 1089.4938 \\ 545.7554 & 1089.4963 & 1089.4938 \\ 455.7133 & 909.4120 & 909.4113 \\ 479.5794 & 1435.7164 & 1435.7168\end{array}$

$\begin{array}{lrr}\text { ppm } & \text { M } & \text { Score } \\ 0.96 & 0 & 53 \\ 1.27 & 0 & 57 \\ 2.08 & 0 & 59 \\ 0.90 & 0 & 99 \\ 2.30 & 0 & 93 \\ 0.74 & 0 & 52 \\ -0.24 & 1 & 51\end{array}$

$\begin{array}{rc}\text { Expect } & \text { Rank } \\ 4.9 e-06 & 1 \\ 2 e-06 & \frac{1}{1} \\ 1.4 e-06 & 1 \\ 1.2 e-10 & 1 \\ 4.9 e-10 & \frac{1}{1} \\ 6.1 e-06 & 1 \\ 7.1 e-06 & 1\end{array}$

Peptide

Ü K.ANNIDFGQLLK.D

U K. ANNIDFGQLLK.D

U K. ANNIDFGQLLK.D

U R.AESGNLEGDAK. T

U R.AESGNLEGDAK.T

U K.AEMETTTK.K

$479.5794 \quad 1435.7164 \quad 1435.7168$

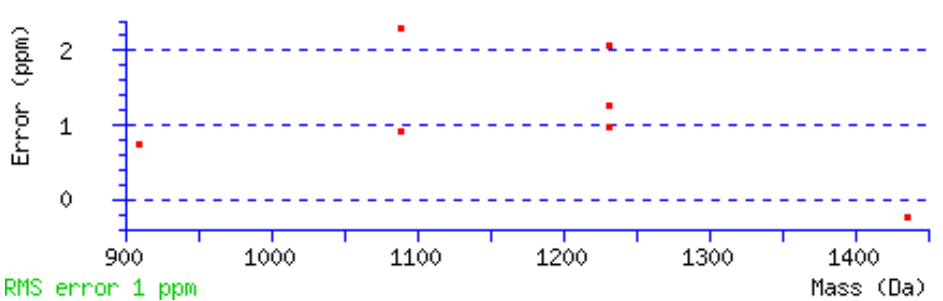




\title{
MASCOT Search Results
}

\section{Protein View: TP84 10}

\author{
Database: TP84 \\ Score: $\quad 1083$ \\ Nominal mass $\left(\mathbf{M}_{\mathbf{r}}\right): 31601$ \\ Calculated pI: $\quad 4.70$
}

Sequence similarity is available as an NCBI BLAST search of 65. against nr.

\section{Search parameters}

\author{
MS data file: \\ Enzyme: \\ O: \FA $\backslash 02-1$ uty2018\80202246zeb_czas30.raw \\ Fixed modifications: \\ Trypsin: cuts C-term side of KR unless next residue is $\mathrm{P}$. \\ Variable modifications: Oxidation (M)
}

\section{Protein sequence coverage: $17 \%$}

Matched peptides shown in bold red.

1 MQDKQITDEF IQKANKASEF GFLQFFAGEG GQQGGEPTDP TDPTDPLDDP

51 EGGEPNDPTD PTDPVDDQDD NDDDTGITYE KGLIDRILKA NNIDFGQLLK 101 DNPELKRQYQ ARFNKNMSKR LEKYQGVDVD EYFELKKRAE SGNLEGDAKT 151 WKEKYEQLKA EMETTTKKTA IQQYAIENGF DSEQIAFITS MIDMNKLERD 201 DEGEWMGIDE EVERIKEKFP RMFEPRDGQV GGTPKKESKY NPGTKKHNQD 251 TKPTDPRELG RQIALQRHKN RLKQQ

Unformatted sequence string: $\mathbf{2 7 5}$ residues (for pasting into other applications).

Sort peptides by $\bullet$ Residue Number $\bigcirc$ Increasing Mass $\bigcirc$ Decreasing Mass

Show predicted peptides also

\begin{tabular}{|c|c|}
\hline Query & start - E \\
\hline$\sigma^{\prime 14691}$ & $90-1$ \\
\hline 14692 & $90-$ \\
\hline 14693 & $90-$ \\
\hline 14694 & $90-1$ \\
\hline$\overline{14695}$ & $90-$ \\
\hline$\nearrow^{\prime} 14696$ & $90-1$ \\
\hline$\underbrace{\prime} 14697$ & $90-$ \\
\hline$\sigma^{\prime} 14698$ & $90-100$ \\
\hline$\widetilde{14699}$ & $90-1$ \\
\hline$\checkmark 14700$ & $90-1$ \\
\hline$\underbrace{\prime 27607}$ & $124-136$ \\
\hline$\complement^{\prime 27608}$ & $124-$ \\
\hline$\triangle 27609$ & $124-136$ \\
\hline$\nearrow^{\prime 2} 27611$ & $124-1$ \\
\hline$\nabla^{\prime} 15263$ & $138-1$ \\
\hline$\nearrow^{\prime 15264}$ & $138-1$ \\
\hline$\sigma^{\prime} 15265$ & $138-$ \\
\hline$\sigma^{\prime} \underline{9432}$ & 139 - \\
\hline$\checkmark \underline{9433}$ & $139-$ \\
\hline 223 & 246 \\
\hline
\end{tabular}

$\begin{array}{rrr}\text { Observed } & \text { Mr (expt) } & \text { Mr (calc) } \\ 616.8344 & 1231.6543 & 1231.6561 \\ 616.8350 & 1231.6555 & 1231.6561 \\ 616.8354 & 1231.6563 & 1231.6561 \\ 616.8354 & 1231.6563 & 1231.6561 \\ 616.8355 & 1231.6565 & 1231.6561 \\ 616.8358 & 1231.6571 & 1231.6561 \\ 616.8358 & 1231.6571 & 1231.6561 \\ 616.8358 & 1231.6571 & 1231.6561 \\ 616.8359 & 1231.6573 & 1231.6561 \\ 616.8360 & 1231.6574 & 1231.6561 \\ 802.8767 & 1603.7388 & 1603.7406 \\ 802.8770 & 1603.7394 & 1603.7406 \\ 802.8777 & 1603.7408 & 1603.7406 \\ 802.8787 & 1603.7429 & 1603.7406 \\ 416.2055 & 1245.5946 & 1245.5949 \\ 416.2056 & 1245.5950 & 1245.5949 \\ 623.8051 & 1245.5956 & 1245.5949 \\ 545.7553 & 1089.4961 & 1089.4938 \\ 545.7561 & 1089.4976 & 1089.4938 \\ 479.5802 & 1435.7187 & 1435.7168\end{array}$

\begin{tabular}{rrr}
\multicolumn{1}{c}{ ppm } & M & Score \\
-1.46 & 0 & 52 \\
-0.45 & 0 & 61 \\
0.16 & 0 & 64 \\
0.20 & 0 & 61 \\
0.33 & 0 & 59 \\
0.85 & 0 & 54 \\
0.85 & 0 & 64 \\
0.86 & 0 & 58 \\
1.01 & 0 & 58 \\
1.11 & 0 & 62 \\
-1.11 & 0 & 83 \\
-0.73 & 0 & 90 \\
0.10 & 0 & 82 \\
1.45 & 0 & 76 \\
-0.26 & 1 & 53 \\
0.098 & 1 & 59 \\
0.56 & 1 & 59 \\
2.10 & 0 & 87 \\
3.47 & 0 & 94 \\
1.37 & 1 & 52
\end{tabular}

Expect Rank

$6 e-06 \quad 1$

$7.3 e-07$

3. $6 e-07$

8. $2 e-07$

1. $3 e-06$

$4.2 e-06$

3. $6 e-07$

1. $5 e-06$

1. $7 e-06$

$6.3 e-07$

4. $5 e-09$

9. $7 e-10$

$6.7 e-09$

$2.3 e-08$

$4.5 e-06$

1. $1 e-06$

1. $4 e-06$

1. $9 e-09$

4. $1 e-10$

$6.5 e-06$
U Peptide

U K.ANNIDFGQLLK. D

U K. ANNIDFGQLLK.D

U K. ANNIDFGQLLK. D

U K. ANNIDFGQLLK. D

U K. ANNIDFGQLLK. D

U K. ANNIDFGQLLK. D

U K.ANNIDFGQLLK. D

U K. ANNIDFGQLLK. D

U K.ANNIDFGQLLK. D

U K. ANNIDFGQLLK.D

U K.YQGVDVDEYFELK. K

U K.YQGVDVDEYFELK.K

U K. YQGVDVDEYFELK. K

U K. YQGVDVDEYFELK. K

U K. RAESGNLEGDAK. T

U K. RAESGNLEGDAK. T

U K. RAESGNLEGDAK. T

U R. AESGNLEGDAK. T

U R. AESGNLEGDAK. T

U K.KHNQDTKPTDPR.E

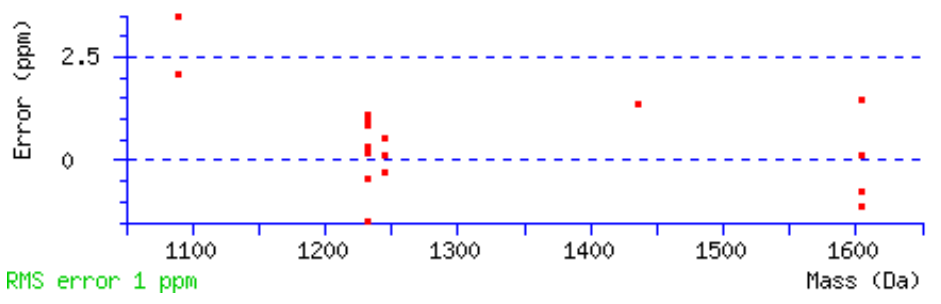




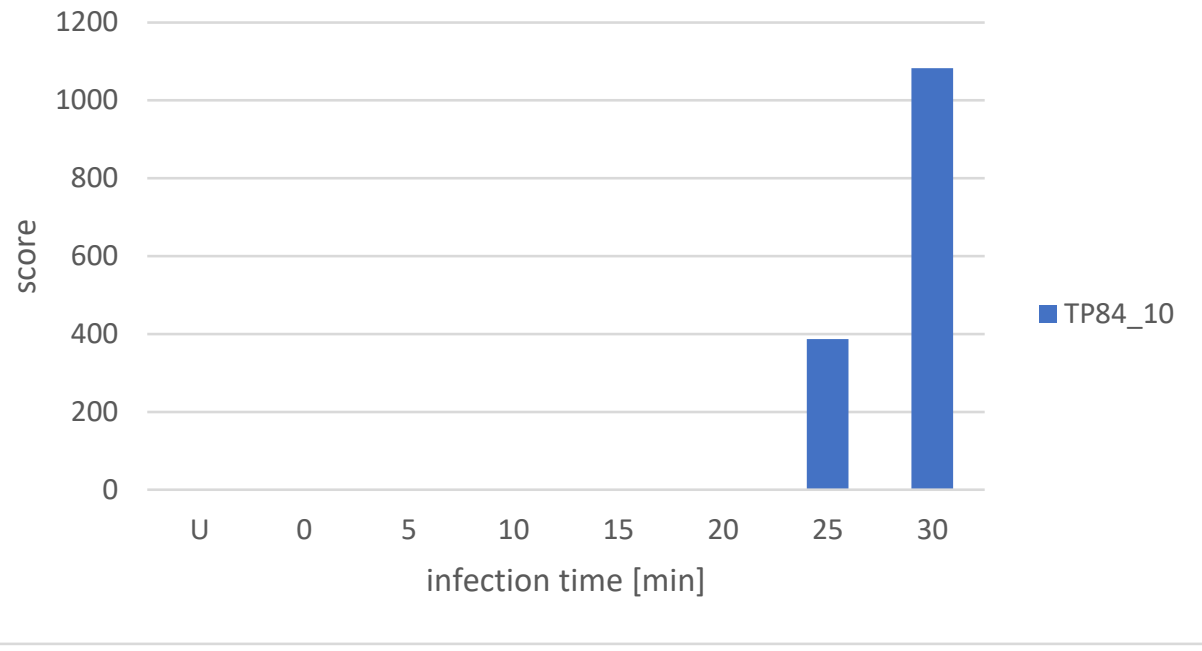

\section{MASCOT Search Results}

\section{Protein View: TP84_11}

$\begin{array}{ll}\text { Database: } & \text { TP84 } \\ \text { Score: } & 341 \\ \text { Nominal mass }\left(M_{\mathbf{r}}\right): & 14016 \\ \text { Calculated pI: } & 5.84\end{array}$

Sequence similarity is available as an NCBI BLAST search of 66 . against $\mathrm{nr}$.

\section{Search parameters}
MS data file:
O: \FA $\backslash 02$-luty2018\80202241zeb_czas5.raw
Enzyme:
Trypsin: cuts C-term side of KR unless next residue is $\mathrm{P}$.
Fixed modifications:
Carbamidomethyl (C)
Variable modifications: Oxidation (M)

\section{Protein sequence coverage: $\mathbf{2 4 \%}$}

Matched peptides shown in bold red.

1 MNLQPKIVSI AGQKEFLATT QGLVHKVGGV TLDASKFTPD ENGFIKAGSA

51 LALTASGKAE PFNVSTPGDP STANGTPYIL AHDVQIKDGT TNIDAVAGVL

101 EAAYLKSSVV TTAEPGRVVV TQDFIDASNG REHLR

Unformatted sequence string: 135 residues (for pasting into other applications).

Sort peptides by $\odot$ Residue Number $\bigcirc$ Increasing Mass $\bigcirc$ Decreasing Mass

Show predicted peptides also

\begin{tabular}{|c|c|c|c|c|c|c|c|c|c|c|c|}
\hline Query & Start - End & Observed & $\operatorname{Mr}(\exp t)$ & $\operatorname{Mr}(\operatorname{calc})$ & ppm & $\mathbf{M}$ & Score & Expect & Rank & $\mathrm{U}$ & Peptide \\
\hline$\mho^{\prime 2} \underline{2916}$ & $27-36$ & 473.7660 & 945.5175 & 945.5131 & 4.60 & $\dddot{0}$ & 52 & $6.4 e-06$ & 1 & $\ddot{\mathrm{U}}$ & K. VGGVTLDASK. F \\
\hline$\triangle \underline{4837}$ & $47-58$ & 523.7960 & 1045.5775 & 1045.5768 & 0.70 & 0 & 79 & $1.3 e-08$ & 1 & $\mathrm{U}$ & K.AGSALALTASGK. A \\
\hline$\checkmark 4839$ & $47-58$ & 523.7974 & 1045.5803 & 1045.5768 & 3.42 & 0 & 86 & $2.6 e-09$ & 1 & $\mathrm{U}$ & K.AGSALALTASGK. A \\
\hline$\sigma^{\prime} \underline{5948}$ & $107-117$ & 552.2900 & 1102.5654 & 1102.5619 & 3.18 & 0 & 84 & $3.6 e-09$ & 1 & U & K.SSVVTTAEPGR.V \\
\hline ه'5956 & $107-117$ & 552.2909 & 1102.5673 & 1102.5619 & 4.98 & 0 & 91 & $7.2 e-10$ & 1 & $\mathrm{U}$ & K.SSVVTTAEPGR.V \\
\hline
\end{tabular}

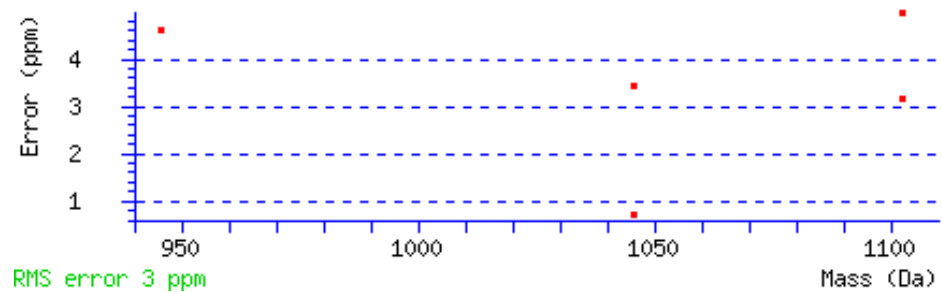




\title{
MASCOT Search Results
}

\section{Protein View: TP84_11}

\author{
Database: TP84 \\ Score: 449 \\ Nominal mass $\left(M_{\mathbf{r}}\right): 14016$ \\ Calculated pI: $\quad 5.84$
}

Sequence similarity is available as an NCBI BLAST search of 66. against nr.

\section{Search parameters}

\author{
MS data file: \\ 0: \FA $\backslash 02$-luty2018\80202242zeb_czas10.raw \\ Enzyme: \\ Trypsin: cuts C-term side of KR unless next residue is $P$. \\ Fixed modifications: \\ Carbamidomethyl (C) \\ Variable modifications: Oxidation (M)
}

\section{Protein sequence coverage: $17 \%$}

Matched peptides shown in bold red.
1 MNLQPKIVSI AGQKEFLATT QGLVHKVGGV TLDASKFTPD ENGFIKAGSA
51 LALTASGKAE PFNVSTPGDP STANGTPYIL AHDVQIKDGT TNIDAVAGVL 101 EAAYLKSSVV TTAEPGRVVV TQDFIDASNG RFHLR

Unformatted sequence string: 135 residues (for pasting into other applications).

Sort peptides by $\bullet$ Residue Number $\bigcirc$ Increasing Mass $\bigcirc$ Decreasing Mass

Show predicted peptides also

\begin{tabular}{|c|c|c|c|c|c|c|c|c|c|c|c|}
\hline Query & Start - End & Observed & $M r(\exp t)$ & $\mathrm{Mr}(\mathrm{calc})$ & ppm & $\mathbf{M}$ & Score & Expect & Rank & $\mathrm{U}$ & Peptide \\
\hline$\checkmark 4756$ & $47-58$ & 523.7957 & 1045.5767 & 1045.5768 & -0.0038 & $\ddot{0}$ & 62 & $6.1 e-07$ & 1 & $\mathrm{U}$ & K. AGSALALTASGK . A \\
\hline$\varangle^{\prime 4757}$ & $47-58$ & 523.7967 & 1045.5788 & 1045.5768 & 1.97 & 0 & 93 & $4.8 e-10$ & 1 & U & K. AGSALALTASGK . A \\
\hline$\varangle \underline{4759}$ & $47-58$ & 523.7970 & 1045.5795 & 1045.5768 & 2.64 & 0 & 85 & $2.8 e-09$ & 1 & $\mathrm{U}$ & K. AGSALALTASGK . A \\
\hline$\underline{4760}$ & $47-58$ & 523.7977 & 1045.5809 & 1045.5768 & 3.99 & 0 & 102 & $5.7 e-11$ & 1 & U & K. AGSALALTASGK . A \\
\hline 5831 & $107-117$ & 552.2892 & 1102.5639 & 1102.5619 & 1.86 & 0 & 85 & $3 e-09$ & 1 & U & K.SSVVTTAEPGR.V \\
\hline$\tau^{\prime 5834}$ & $107-117$ & 552.2899 & 1102.5651 & 1102.5619 & 2.98 & 0 & 85 & $3 e-09$ & 1 & $\mathrm{U}$ & K.SSVVTTAEPGR.V \\
\hline
\end{tabular}

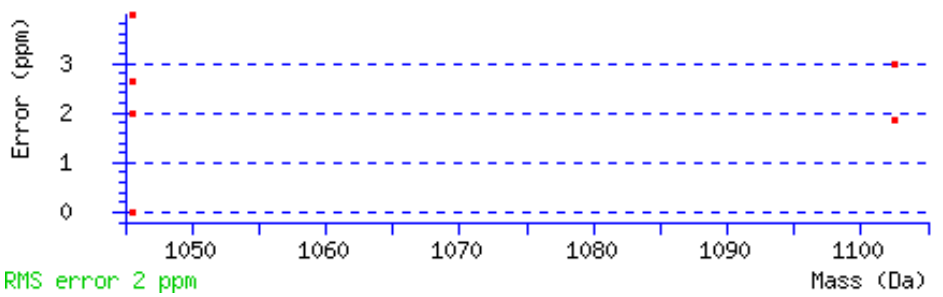




\title{
MASCOT Search Results
}

\section{Protein View: TP84 11}

\author{
Database: TP84 \\ Score: 606 \\ Nominal mass $\left(M_{\mathbf{r}}\right): 14016$ \\ Calculated pI: $\quad 5.84$
}

Sequence similarity is available as an NCBI BLAST search of 66. against nr.

\section{Search parameters}

\author{
MS data file: \\ $0: \backslash F A \backslash 02-l u t y 2018 \backslash 80202243 z e b$ czas15.raw \\ Enzyme: \\ Trypsin: cuts C-term side of KR unless next residue is $\mathrm{P}$. \\ Fixed modifications: \\ Carbamidomethyl (C) \\ Variable modifications: Oxidation (M)
}

\section{Protein sequence coverage: $33 \%$}

Matched peptides shown in bold red.

1 MNLQPKIVSI AGQKEFLATT QGLVHKVGGV TLDASKFTPD ENGFIKAGSA

51 LALTASGKAE PFNVSTPGDP STANGTPYIL AHDVQIKDGT TNIDAVAGVL

101 EAAYLKSSVV TTAEPGRVVV TQDFIDASNG RFHLR

Unformatted sequence string: 135 residues (for pasting into other applications).

Sort peptides by $\odot$ Residue Number $\bigcirc$ Increasing Mass $\bigcirc$ Decreasing Mass

Show predicted peptides also

\begin{tabular}{|c|c|c|c|c|c|c|c|c|c|c|c|}
\hline Query & Start - End & Observed & $M r(\exp t)$ & $\operatorname{Mr}(\mathrm{calc})$ & ppm & $\mathbf{M}$ & Score & Expect & Rank & $\mathrm{U}$ & Peptide \\
\hline$\nabla^{\prime 11185}$ & $15-26$ & 448.5821 & 1342.7245 & 1342.7245 & -0.00074 & 0 & 49 & $1.2 e-05$ & 1 & $\mathrm{U}$ & K. EFLATTQGLVHK.V \\
\hline$\checkmark 2852$ & $27-36$ & 473.7643 & 945.5140 & 945.5131 & 0.96 & 0 & 79 & $1.2 e-08$ & $\dddot{1}$ & $\mathrm{U}$ & K.VGGVTLDASK. F \\
\hline$\triangle^{\prime 4} \underline{756}$ & $47-58$ & 523.7964 & 1045.5783 & 1045.5768 & 1.51 & 0 & 104 & $3.7 e-11$ & 1 & $\mathrm{U}$ & K. AGSALALTASGK. A \\
\hline$\triangle^{\prime 4} \underline{757}$ & $47-58$ & 523.7966 & 1045.5787 & 1045.5768 & 1.85 & 0 & 94 & $3.6 e-10$ & 1 & $\mathrm{U}$ & K. AGSALALTASGK. A \\
\hline$\square^{\prime 4} 758$ & $47-58$ & 523.7973 & 1045.5801 & 1045.5768 & 3.23 & 0 & 95 & $3 e-10$ & 1 & $\mathrm{U}$ & K. AGSALALTASGK. A \\
\hline$\nearrow^{\prime} \underline{5781}$ & $107-117$ & 552.2887 & 1102.5628 & 1102.5619 & 0.90 & 0 & 92 & $6.7 e-10$ & 1 & $\mathrm{~J}$ & K.SSVVTTAEPGR.V \\
\hline$\square^{\prime} 5788$ & $107-117$ & 552.2896 & 1102.5646 & 1102.5619 & 2.53 & 0 & 92 & $6.7 e-10$ & 1 & $\mathrm{~J}$ & K.SSVVTTAEPGR.V \\
\hline$\nearrow^{\prime} 5790$ & $107-117$ & 552.2902 & 1102.5659 & 1102.5619 & 3.64 & 0 & 92 & $7.1 e-10$ & 1 & U & K.SSVVTTAEPGR.V \\
\hline
\end{tabular}

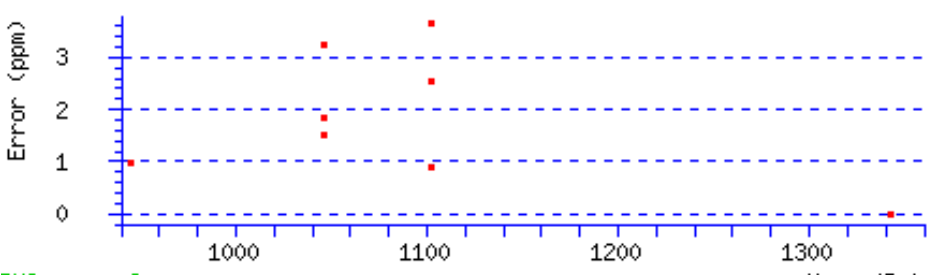

RMS error 2 ppm

Mass (Da) 


\title{
MASCOT Search Results
}

\section{Protein View: TP84 11}

\author{
Database: TP84 \\ Score: 1304 \\ Nominal mass $\left(M_{\mathbf{r}}\right): 14016$ \\ Calculated pI: $\quad 5.84$
}

Sequence similarity is available as an NCBI BLAST search of 66. against nr.

\section{Search parameters}

MS data file:

Enzyme:

Fixed modifications:

Variable modifications

\section{Protein sequence coverage: $\mathbf{3 0} \%$}

Matched peptides shown in bold red.

1 MNLQPKIVSI AGQKEFLATT QGLVHKVGGV TLDASKFTPD ENGFIKAGSA

51 LALTASGKAE PFNVSTPGDP STANGTPYIL AHDVQIKDGT TNIDAVAGVL 101 EAAYLKSSVV TTAEPGRVVV TQDFIDASNG RFHLR

Unformatted sequence string: 135 residues (for pasting into other applications).

Sort peptides by $\odot$ Residue Number $\bigcirc$ Increasing Mass $\bigcirc$ Decreasing Mass

Show predicted peptides also

\begin{tabular}{|c|c|}
\hline Query & Start - End \\
\hline$\checkmark 1952$ & $7-14$ \\
\hline 20854 & $15-26$ \\
\hline 20855 & $15-26$ \\
\hline 20856 & $15-26$ \\
\hline ¿20857 & $15-26$ \\
\hline$\checkmark 5673$ & $27-36$ \\
\hline$\checkmark 5674$ & $27-36$ \\
\hline$\nearrow^{\prime 5675}$ & $27-36$ \\
\hline$\triangleleft^{\prime} \underline{676}$ & $27-36$ \\
\hline$\smile^{\prime 5677}$ & $27-36$ \\
\hline$\triangleleft^{\prime} 5678$ & $27-36$ \\
\hline$\overline{5680}$ & $27-36$ \\
\hline$\checkmark 5681$ & $27-36$ \\
\hline$\nearrow^{\prime} \underline{682}$ & $27-36$ \\
\hline$\nearrow^{\prime} \underline{683}$ & $27-36$ \\
\hline$\nearrow^{\prime} \underline{684}$ & $27-36$ \\
\hline$\nearrow^{\prime} 11487$ & $107-117$ \\
\hline$\sigma^{\prime} 11488$ & $107-117$ \\
\hline$\checkmark 11489$ & $107-117$ \\
\hline$\triangle^{\prime} 11490$ & $107-117$ \\
\hline & 107 \\
\hline
\end{tabular}

$\begin{array}{rrr}\text { Observed } & \text { Mr (expt) } & \text { Mr (calc) } \\ 408.2545 & 814.4944 & 814.4912 \\ 448.5838 & 1342.7295 & 1342.7245 \\ 448.5839 & 1342.7298 & 1342.7245 \\ 672.3726 & 1342.7307 & 1342.7245 \\ 672.3729 & 1342.7313 & 1342.7245 \\ 473.7648 & 945.5151 & 945.5131 \\ 473.7655 & 945.5165 & 945.5131 \\ 473.7656 & 945.5166 & 945.5131 \\ 473.7659 & 945.5173 & 945.5131 \\ 473.7660 & 945.5174 & 945.5131 \\ 473.7661 & 945.5175 & 945.5131 \\ 473.7662 & 945.5179 & 945.5131 \\ 473.7665 & 945.5184 & 945.5131 \\ 473.7666 & 945.5186 & 945.5131 \\ 473.7667 & 945.5188 & 945.5131 \\ 473.7671 & 945.5196 & 945.5131 \\ 552.2913 & 1102.5680 & 1102.5619 \\ 552.2915 & 1102.5685 & 1102.5619 \\ 552.2916 & 1102.5686 & 1102.5619 \\ 552.2917 & 1102.5688 & 1102.5619 \\ 552.2922 & 1102.5699 & 1102.5619\end{array}$

$\begin{array}{llc}\text { ppm } & \text { M } & \text { Score } \\ 3.86 & 0 & 51 \\ 3.71 & 0 & 84 \\ 3.95 & 0 & 81 \\ 4.62 & 0 & 83 \\ 5.08 & 0 & 80 \\ 2.10 & 0 & 76 \\ 3.60 & 0 & 75 \\ 3.64 & 0 & 74 \\ 4.47 & 0 & 74 \\ 4.55 & 0 & 71 \\ 4.68 & 0 & 59 \\ 5.06 & 0 & 58 \\ 5.61 & 0 & 56 \\ 5.84 & 0 & 54 \\ 6.01 & 0 & 71 \\ 6.86 & 0 & 75 \\ 5.60 & 0 & 92 \\ 6.05 & 0 & 92 \\ 6.14 & 0 & 92 \\ 6.34 & 0 & 92 \\ 7.34 & 0 & 76\end{array}$
$1 e-06$ $4.5 e-09$

$7.4 e-09$

$5.5 e-09$

$1 e-08$

2. $7 e-08$

$3 e-08$

$4.2 e-08$

4. $4 \mathrm{e}-08$

$8.5 e-08$

1. $3 e-06$

$1.6 e-06$

2. $3 e-06$

3. $6 e-06$

8. $2 e-08$

$3 e-08$

6. $8 \mathrm{e}-10$

$6.7 e-10$

6. $6 e-10$

6. $7 e-10$

2. $5 e-08$
U Peptide

U K. IVSIAGQK.E

U K.EFLATTQGLVHK.V

U K.EFLATTQGLVHK.V

U K.EFLATTQGLVHK.V

U K. EFLATTOGLVHK.V

U K.VGGVTLDASK. F

U K.VGGVTLDASK. F

U K. VGGVTLDASK. F

U K.VGGVTLDASK. F

U K.VGGVTLDASK. F

U K.VGGVTLDASK. $F$

U K.VGGVTLDASK. F

U K.VGGVTLDASK. F

U K.VGGVTLDASK. F

U K. VGGVTLDASK . F

U K.VGGVTLDASK. F

U K.SSVVTTAEPGR.V

U K.SSVVTTAEPGR.V

U K.SSVVTTAEPGR.V

U K.SSVVTTAEPGR.V

U K.SSVVTTAEPGR.V

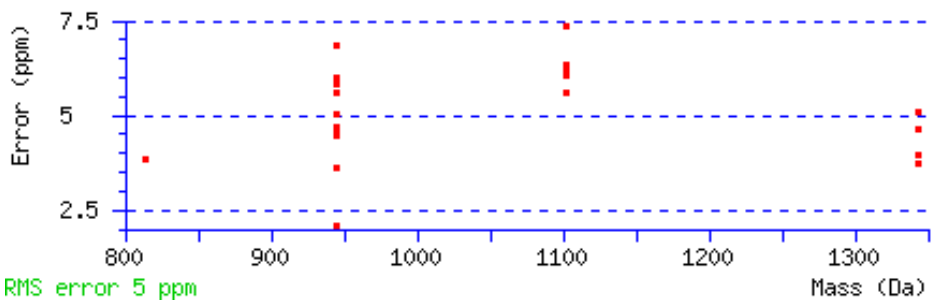

Mascot: http://www.matrixscience.com/ 


\title{
MASCOT Search Results
}

\section{Protein View: TP84_11}

$\begin{array}{ll}\text { Database: } & \text { TP84 } \\ \text { Score: } & 1935 \\ \text { Nominal mass (Mr): } & 14016 \\ \text { Calculated pI: } & 5.84\end{array}$

Sequence similarity is available as an NCBI BLAST search of 66 . against nr.

\section{Search parameters}

\author{
MS data file: \\ Enzyme: \\ O: \FA $\backslash 02-1$ uty2018\80202245zeb_czas25.raw \\ Fixed modifications: $\quad$ Carbamidomethyl (C) \\ Variable modifications: Oxidation (M)
}

\section{Protein sequence coverage: $\mathbf{7 4 \%}$}

Matched peptides shown in bold red.

1 MNLQPKIVSI AGQKEFLATT QGLVHKVGGV TLDASKFTPD ENGFIKAGSA

51 LALTASGKAE PFNVSTPGDP STANGTPYIL AHDVQIKDGT TNIDAVAGVL

101 EAAYLKSSVV TTAEPGRVVV TQDFIDASNG RFHLR

Unformatted sequence string: 135 residues (for pasting into other applications).

Sort peptides by $\odot$ Residue Number $\bigcirc$ Increasing Mass $\bigcirc$ Decreasing Mass

Show predicted peptides also

\begin{tabular}{|c|c|c|c|c|}
\hline Query & Start - End & Observed & $M r(\exp t)$ & $\operatorname{Mr}$ (calc) \\
\hline$\triangle 1292$ & $7-14$ & 408.2525 & 814.4904 & 814.4912 \\
\hline$\checkmark 13580$ & $15-26$ & 448.5822 & 1342.7246 & 1342.7245 \\
\hline$\checkmark 13581$ & $15-26$ & 448.5822 & 1342.7246 & 1342.7245 \\
\hline 13582 & $15-26$ & 448.5823 & 1342.7251 & 1342.7245 \\
\hline$\checkmark \underline{3595}$ & $27-36$ & 473.7606 & 945.5067 & 945.5131 \\
\hline$\triangle^{\prime 3596}$ & $27-36$ & 473.7635 & 945.5125 & 945.5131 \\
\hline$\checkmark 3597$ & $27-36$ & 473.7636 & 945.5127 & 945.5131 \\
\hline$\underline{3598}$ & $27-36$ & 473.7636 & 945.5127 & 945.5131 \\
\hline$\checkmark 3599$ & $27-36$ & 473.7638 & 945.5130 & 945.5131 \\
\hline$\triangle \underline{3600}$ & $27-36$ & 473.7639 & 945.5132 & 945.5131 \\
\hline$\underline{3601}$ & $27-36$ & 473.7640 & 945.5134 & 945.5131 \\
\hline$\underline{3602}$ & $27-36$ & 473.7643 & 945.5141 & 945.5131 \\
\hline$\triangle^{5} \underline{964}$ & $47-58$ & 523.7958 & 1045.5771 & 1045.5768 \\
\hline$\checkmark \underline{5965}$ & $47-58$ & 523.7959 & 1045.5773 & 1045.5768 \\
\hline$\nearrow^{\prime} 5966$ & $47-58$ & 523.7960 & 1045.5774 & 1045.5768 \\
\hline$\underline{5968}$ & $47-58$ & 523.7961 & 1045.5777 & 1045.5768 \\
\hline$\checkmark \underline{5969}$ & $47-58$ & 523.7962 & 1045.5779 & 1045.5768 \\
\hline$\nearrow^{\prime} \underline{9970}$ & $47-58$ & 523.7962 & 1045.5779 & 1045.5768 \\
\hline$\sigma^{\prime 36469}$ & $59-87$ & 1013.8376 & 3038.4910 & 3038.4985 \\
\hline$\varangle^{\prime 36470}$ & $59-87$ & 1013.8377 & 3038.4913 & 3038.4985 \\
\hline$\mho^{\prime} 36471$ & $59-87$ & 1013.8383 & 3038.4931 & 3038.4985 \\
\hline$\mho^{\prime} 36472$ & $59-87$ & 1013.8385 & 3038.4937 & 3038.4985 \\
\hline$\checkmark 26795$ & $88-106$ & 641.0018 & 1919.9835 & 1919.9840 \\
\hline$\triangle 7174$ & $107-117$ & 552.2880 & 1102.5615 & 1102.5619 \\
\hline$\triangle 7175$ & $107-117$ & 552.2882 & 1102.5618 & 1102.5619 \\
\hline 77 & 107 & 552.2886 & 1102.5626 & 1102.5619 \\
\hline
\end{tabular}

\begin{tabular}{|c|c|c|c|c|c|c|}
\hline $\mathrm{ppm}$ & M & Score & Expect & Rank & $\mathrm{U}$ & Peptide \\
\hline-1.02 & $\ddot{0}$ & 54 & $3.9 e-06$ & 1 & $\ddot{\mathrm{U}}$ & K. IVSIAGQK.E \\
\hline 0.089 & 0 & 72 & $6.7 e-08$ & 1 & $\mathrm{U}$ & K. EFLATTQGLVHK.V \\
\hline 0.089 & 0 & 57 & $1.8 e-06$ & 1 & $\mathrm{U}$ & K. EFLATTQGLVHK.V \\
\hline 0.42 & 0 & 70 & $1 e-07$ & 1 & $\mathrm{U}$ & K.EFLATTQGLVHK.V \\
\hline-6.74 & 0 & 59 & $1.3 e-06$ & 1 & $\mathrm{U}$ & K. VGGVTLDASK. F \\
\hline-0.65 & 0 & 90 & $1.1 e-09$ & 1 & $\mathrm{U}$ & K. VGGVTLDASK. F \\
\hline-0.42 & 0 & 77 & $1.8 e-08$ & 1 & $\mathrm{U}$ & K.VGGVTLDASK. F \\
\hline-0.40 & 0 & 92 & $5.9 e-10$ & 1 & $\mathrm{U}$ & K. VGGVTLDASK. F \\
\hline-0.14 & 0 & 80 & $9.7 e-09$ & 1 & $\mathrm{U}$ & K. VGGVTLDASK. F \\
\hline 0.13 & 0 & 80 & $9.4 e-09$ & 1 & $\mathrm{U}$ & K. VGGVTLDASK. F \\
\hline 0.26 & 0 & 88 & $1.7 e-09$ & 1 & $\mathrm{U}$ & K. VGGVTLDASK. F \\
\hline 1.08 & 0 & 67 & $1.9 e-07$ & 1 & $\mathrm{U}$ & K. VGGVTLDASK. F \\
\hline 0.36 & 0 & 96 & $2.5 e-10$ & 1 & $\mathrm{U}$ & K. AGSALALTASGK. A \\
\hline 0.55 & 0 & 95 & $3.1 e-10$ & 1 & $\mathrm{U}$ & K. AGSALALTASGK. A \\
\hline 0.59 & 0 & 96 & $2.5 e-10$ & 1 & $\mathrm{U}$ & K. AGSALALTASGK. A \\
\hline 0.93 & 0 & 104 & $4.3 e-11$ & 1 & $\mathrm{U}$ & K. AGSALALTASGK. A \\
\hline 1.07 & 0 & 102 & $6.3 e-11$ & 1 & $\mathrm{U}$ & K. AGSALALTASGK. A \\
\hline 1.11 & 0 & 70 & $9.5 e-08$ & 1 & $\mathrm{U}$ & K. AGSALALTASGK. A \\
\hline-2.49 & 0 & 116 & $2.3 e-12$ & 1 & $\mathrm{U}$ & K. AEPFNVSTPGDPSTANGTPYILAHDVQIK.D \\
\hline-2.39 & 0 & 106 & $2.6 e-11$ & 1 & $\mathrm{U}$ & K. AEPFNVSTPGDPSTANGTPYILAHDVQIK. D \\
\hline-1.80 & 0 & 120 & $1.1 e-12$ & 1 & $\mathrm{U}$ & K. AEPFNVSTPGDPSTANGTPYILAHDVQIK.D \\
\hline-1.60 & 0 & 99 & $1.2 e-10$ & 1 & $\mathrm{U}$ & K. AEPFNVSTPGDPSTANGTPYILAHDVQIK.D \\
\hline-0.27 & 0 & 93 & $4.8 e-10$ & 1 & $\mathrm{U}$ & K.DGTTNIDAVAGVLEAAYLK.S \\
\hline-0.35 & 0 & 92 & $6.6 e-10$ & 1 & $\mathrm{U}$ & K.SSVVTTAEPGR.V \\
\hline 0.083 & 0 & 92 & $6.5 e-10$ & 1 & $\mathrm{U}$ & K.SSVVTTAEPGR.V \\
\hline 0.72 & 0 & 92 & $6.6 e-10$ & 1 & $\mathrm{U}$ & K.SSVVTTAEPGR.V \\
\hline
\end{tabular}

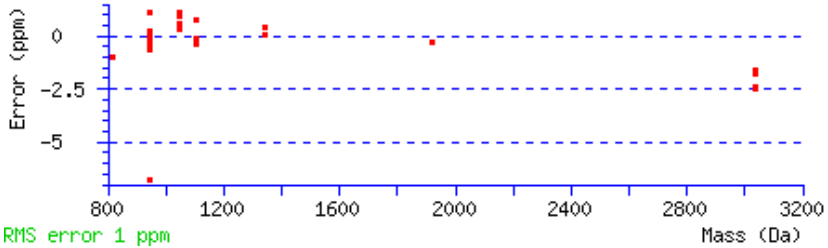


MASCOT Search Results

\title{
Protein View: TP84_11
}

\author{
Database: $\quad$ TP84 \\ Score: $\quad 4174$ \\ Nominal mass (Mr): 14016 \\ Calculated pI: $\quad 5.84$
}

Sequence similarity is available as an NCBI BLAST search of 66 . against nr.

\section{Search parameters}

\author{
MS data file: \\ Enzyme: \\ 0: \FA $\backslash 02-$ luty2018\80202246zeb_czas30.raw \\ Fixed modifications: \\ Trypsin: cuts C-term side of KR unless next residue is $P$. \\ Variable modifications: Oxidation (M)
}

\section{Protein sequence coverage: $\mathbf{8 5 \%}$}

Matched peptides shown in bold red.

1 MNLQPKIVSI AGQKEFLATT QGLVHKVGGV TLDASKFTPD ENGFIKAGSA

51 LALTASGKAE PFNVSTPGDP STANGTPYIL AHDVQIKDGT TNIDAVAGVL

101 EAAYLKSSVV TTAEPGRVVV TQDFIDASNG RFHLR

Unformatted sequence string: 135 residues (for pasting into other applications).

Sort peptides by $\odot$ Residue Number $\bigcirc$ Increasing Mass $\bigcirc$ Decreasing Mass

Show predicted peptides also

\begin{tabular}{|c|c|c|c|c|}
\hline Query & Start - End & Observed & $M r($ expt $)$ & $\mathrm{Mr}$ (calc) \\
\hline$\nearrow^{\prime 1539}$ & $7-14$ & 408.2524 & 814.4902 & 814.4912 \\
\hline$\checkmark 1543$ & $7-14$ & 408.2529 & 814.4913 & 814.4912 \\
\hline$\checkmark 1 \overline{19130}$ & $15-26$ & 448.5815 & 1342.7226 & 1342.7245 \\
\hline$\underbrace{\prime 19131}$ & $15-26$ & 448.5815 & 1342.7228 & 1342.7245 \\
\hline$\sigma^{\prime} 19132$ & $15-26$ & 672.3687 & 1342.7228 & 1342.7245 \\
\hline$\checkmark 19133$ & $15-26$ & 448.5818 & 1342.7235 & 1342.7245 \\
\hline 19134 & $15-26$ & 672.3692 & 1342.7239 & 1342.7245 \\
\hline$\overline{19135}$ & $15-26$ & 448.5819 & 1342.7240 & 1342.7245 \\
\hline$\checkmark 19136$ & $15-26$ & 448.5820 & 1342.7242 & 1342.7245 \\
\hline 19137 & $15-26$ & 448.5820 & 1342.7243 & 1342.7245 \\
\hline$\varpi^{\prime} 19138$ & $15-26$ & 672.3696 & 1342.7246 & 1342.7245 \\
\hline$\overline{19139}$ & $15-26$ & 448.5822 & 1342.7248 & 1342.7245 \\
\hline$\checkmark 19140$ & $15-26$ & 672.3698 & 1342.7250 & 1342.7245 \\
\hline$\overline{19141}$ & $15-26$ & 448.5824 & 1342.7254 & 1342.7245 \\
\hline$\checkmark 19142$ & $15-26$ & 672.3700 & 1342.7255 & 1342.7245 \\
\hline$\overline{19143}$ & $15-26$ & 672.3703 & 1342.7261 & 1342.7245 \\
\hline$\checkmark 19144$ & $15-26$ & 672.3703 & 1342.7261 & 1342.7245 \\
\hline$\checkmark 4 \underline{4565}$ & $27-36$ & 473.7636 & 945.5127 & 945.5131 \\
\hline$\triangle \underline{4566}$ & $27-36$ & 473.7637 & 945.5129 & 945.5131 \\
\hline$\nearrow^{\prime 4} 4567$ & $27-36$ & 473.7638 & 945.5130 & 945.5131 \\
\hline$\checkmark 4568$ & $27-36$ & 473.7639 & 945.5132 & 945.5131 \\
\hline$\longdiv { 4 5 6 9 }$ & $27-36$ & 473.7640 & 945.5135 & 945.5131 \\
\hline$\checkmark \underline{4570}$ & $27-36$ & 473.7641 & 945.5136 & 945.5131 \\
\hline$\overline{4571}$ & $27-36$ & 473.7641 & 945.5137 & 945.5131 \\
\hline$\longdiv { 4 5 7 2 }$ & $27-36$ & 473.7644 & 945.5142 & 945.5131 \\
\hline$\checkmark 4573$ & $27-36$ & 473.7644 & 945.5143 & 945.5131 \\
\hline$\triangle^{\prime 7964}$ & $47-58$ & 523.7956 & 1045.5766 & 1045.5768 \\
\hline$\checkmark 7965$ & $47-58$ & 523.7957 & 1045.5768 & 1045.5768 \\
\hline$\overline{7966}$ & $47-58$ & 523.7957 & 1045.5769 & 1045.5768 \\
\hline$\underbrace{\prime 7967}$ & $47-58$ & 523.7958 & 1045.5771 & 1045.5768 \\
\hline$\checkmark \underline{7968}$ & $47-58$ & 523.7958 & 1045.5771 & 1045.5768 \\
\hline$\overline{7969}$ & $47-58$ & 523.7959 & 1045.5773 & 1045.5768 \\
\hline$\checkmark \underline{7970}$ & $47-58$ & 523.7959 & 1045.5773 & 1045.5768 \\
\hline$\checkmark \underline{7971}$ & $47-58$ & 523.7960 & 1045.5774 & 1045.5768 \\
\hline$\checkmark 7972$ & $47-58$ & 523.7961 & 1045.5777 & 1045.5768 \\
\hline$\checkmark \underline{7973}$ & $47-58$ & 523.7962 & 1045.5778 & 1045.5768 \\
\hline$\checkmark 7974$ & $47-58$ & 523.7962 & 1045.5779 & 1045.5768 \\
\hline$\longdiv { 7 9 7 5 }$ & $47-58$ & 523.7962 & 1045.5779 & 1045.5768 \\
\hline$\checkmark 7976$ & $47-58$ & 523.7963 & 1045.5780 & 1045.5768 \\
\hline$\overline{7977}$ & $47-58$ & 523.7965 & 1045.5785 & 1045.5768 \\
\hline 7978 & $47-58$ & 523.7966 & 1045.5785 & 1045.5768 \\
\hline$\checkmark \underline{7979}$ & $47-58$ & 523.7966 & 1045.5786 & 1045.5768 \\
\hline 7980 & $47-58$ & 523.7966 & 1045.5787 & 1045.5768 \\
\hline$\checkmark 7981$ & $47-58$ & 523.7968 & 1045.5790 & 1045.5768 \\
\hline$\nabla^{4} \underline{6599}$ & $59-87$ & 1013.8386 & 3038.4940 & 3038.4985 \\
\hline$\triangle \underline{46600}$ & $59-87$ & 1013.8396 & 3038.4970 & 3038.4985 \\
\hline$\nabla^{4} \underline{6601}$ & $59-87$ & 1013.8402 & 3038.4988 & 3038.4985 \\
\hline$\nearrow^{\prime 35818}$ & $88-106$ & 641.0026 & 1919.9859 & 1919.9840 \\
\hline
\end{tabular}

\begin{tabular}{|c|c|c|c|c|c|c|}
\hline ppm & $\mathbf{M}$ & Score & Expect & Rank & U & Peptide \\
\hline-1.22 & $\ddot{0}$ & 61 & $8.3 e-07$ & 1 & $\overline{\mathrm{U}}$ & K. IVSIAGQK.E \\
\hline 0.13 & 0 & 60 & $1.1 e-06$ & 1 & $\mathrm{U}$ & K. IVSIAGQK.E \\
\hline-1.41 & 0 & 76 & $2.7 e-08$ & 1 & $\mathrm{U}$ & K. EFLATTQGLVHK.V \\
\hline-1.25 & 0 & 75 & $3.5 e-08$ & 1 & U & K.EFLATTQGLVHK.V \\
\hline-1.23 & 0 & 80 & $9.4 e-09$ & 1 & $\mathrm{U}$ & K. EFLATTQGLVHK.V \\
\hline-0.78 & 0 & 78 & $1.4 e-08$ & 1 & $\mathrm{U}$ & K. EFLATTQGLVHK.V \\
\hline-0.41 & 0 & 77 & $1.8 e-08$ & 1 & $\mathrm{U}$ & K.EFLATTQGLVHK.V \\
\hline-0.40 & 0 & 83 & $5.6 e-09$ & 1 & $\mathrm{U}$ & K.EFLATTQGLVHK.V \\
\hline-0.25 & 0 & 75 & $3.1 e-08$ & 1 & $\mathrm{U}$ & K.EFLATTQGLVHK.V \\
\hline-0.16 & 0 & 77 & $2.1 e-08$ & 1 & $\mathrm{U}$ & K. EFLATTQGLVHK.V \\
\hline 0.063 & 0 & 83 & $5.5 e-09$ & 1 & $\mathrm{U}$ & K. EFLATTQGLVHK.V \\
\hline 0.25 & 0 & 74 & $4.4 e-08$ & 1 & $\mathrm{U}$ & K.EFLATTQGLVHK.V \\
\hline 0.41 & 0 & 83 & $5.3 e-09$ & 1 & $\mathrm{U}$ & K. EFLATTQGLVHK.V \\
\hline 0.65 & 0 & 75 & $3.4 e-08$ & 1 & $\mathrm{U}$ & K. EFLATTQGLVHK.V \\
\hline 0.72 & 0 & 78 & $1.8 e-08$ & 1 & $\mathrm{U}$ & K. EFLATTQGLVHK.V \\
\hline 1.17 & 0 & 80 & $1.1 e-08$ & 1 & $\mathrm{U}$ & K. EFLATTQGLVHK.V \\
\hline 1.21 & 0 & 63 & $5.6 e-07$ & 1 & $\mathrm{U}$ & K. EFLATTQGLVHK.V \\
\hline-0.46 & 0 & 78 & $1.5 e-08$ & 1 & $\mathrm{U}$ & K. VGGVTLDASK. F \\
\hline-0.27 & 0 & 76 & $2.6 e-08$ & 1 & $\mathrm{U}$ & K. VGGVTLDASK . F \\
\hline 0.079 & 0 & 63 & $5.2 e-07$ & 1 & $\mathrm{U}$ & K. VGGVTLDASK. F \\
\hline 0.090 & 0 & 75 & $2.9 e-08$ & 1 & $\mathrm{U}$ & K.VGGVTLDASK. F \\
\hline 0.41 & 0 & 71 & $8.3 e-08$ & 1 & $\mathrm{U}$ & K.VGGVTLDASK. F \\
\hline 0.53 & 0 & 77 & $1.8 e-08$ & 1 & $\mathrm{U}$ & K. VGGVTLDASK. F \\
\hline 0.64 & 0 & 82 & $6.9 e-09$ & 1 & $\mathrm{U}$ & K.VGGVTLDASK. F \\
\hline 1.15 & 0 & 64 & $3.7 e-07$ & 1 & $\mathrm{U}$ & K.VGGVTLDASK. F \\
\hline 1.23 & 0 & 82 & $5.8 e-09$ & 1 & $\mathrm{U}$ & K.VGGVTLDASK. F \\
\hline-0.16 & 0 & 84 & $3.7 e-09$ & 1 & $\mathrm{U}$ & K. AGSALALTASGK. A \\
\hline 0.015 & 0 & 84 & $4.3 e-09$ & 1 & $\mathrm{U}$ & K. AGSALALTASGK. A \\
\hline 0.19 & 0 & 105 & $3 e-11$ & 1 & $\mathrm{U}$ & K. AGSALALTASGK. A \\
\hline 0.34 & 0 & 90 & $1.1 e-09$ & 1 & $\mathrm{U}$ & K.AGSALALTASGK. A \\
\hline 0.38 & 0 & 85 & $2.9 e-09$ & 1 & $\mathrm{U}$ & K. AGSALALTASGK . A \\
\hline 0.49 & 0 & 103 & $4.7 e-11$ & 1 & $\mathrm{U}$ & K. AGSALALTASGK. A \\
\hline 0.57 & 0 & 83 & $4.7 e-09$ & 1 & $\mathrm{U}$ & K. AGSALALTASGK. A \\
\hline 0.61 & 0 & 85 & $2.9 e-09$ & 1 & $\mathrm{U}$ & K. AGSALALTASGK. A \\
\hline 0.90 & 0 & 85 & $3 e-09$ & 1 & $\mathrm{U}$ & K. AGSALALTASGK. A \\
\hline 0.99 & 0 & 87 & $1.9 e-09$ & 1 & $\mathrm{U}$ & K. AGSALALTASGK. A \\
\hline 1.11 & 0 & 96 & $2.6 e-10$ & 1 & $\mathrm{U}$ & K. AGSALALTASGK. A \\
\hline 1.14 & 0 & 87 & $2.1 e-09$ & 1 & $\mathrm{U}$ & K. AGSALALTASGK. A \\
\hline 1.20 & 0 & 84 & $4.3 e-09$ & 1 & $\mathrm{U}$ & K. AGSALALTASGK. A \\
\hline 1.66 & 0 & 86 & $2.4 e-09$ & 1 & $\mathrm{U}$ & K. AGSALALTASGK. A \\
\hline 1.72 & 0 & 99 & $1.4 e-10$ & 1 & U & K. AGSALALTASGK. A \\
\hline 1.74 & 0 & 86 & $2.3 e-09$ & 1 & $\mathrm{U}$ & K.AGSALALTASGK. A \\
\hline 1.85 & 0 & 84 & $4.2 e-09$ & 1 & $\mathrm{U}$ & K.AGSALALTASGK. A \\
\hline 2.18 & 0 & 75 & $3 e-08$ & 1 & $\mathrm{U}$ & K.AGSALALTASGK. A \\
\hline-1.50 & 0 & 126 & $2.4 e-13$ & 1 & $\mathrm{U}$ & K.AEPFNVSTPGDPSTANGTPYILAHDVQIK.D \\
\hline-0.51 & 0 & 91 & $7.6 e-10$ & 1. & $\mathrm{U}$ & K. AEPFNVSTPGDPSTANGTPYILAHDVQIK.D \\
\hline 0.078 & 0 & 109 & $1.4 e-11$ & 1 & $\mathrm{U}$ & K. AEPFNVSTPGDPSTANGTPYILAHDVQIK.D \\
\hline 1.01 & 0 & 113 & $5.6 e-12$ & 1 & $\mathrm{U}$ & K. DGTTNIDAVAGVLEAAYLK.S \\
\hline
\end{tabular}


Query Start - End

$\checkmark 35819 \quad 88-106$

$\checkmark 35820 \quad 88-106$

$\checkmark 9849 \quad 107-117$

$\checkmark \mathbf{9 8 5 0} \quad 107-117$

$\checkmark \mathbf{9 8 5 1} \quad 107-117$

$\checkmark 9853 \quad 107-117$

$\varangle \mathbf{9 8 5 5} \quad 107-117$

$\varangle \underline{25079} \quad 118-131$

$\checkmark 25080 \quad 118-131$

$\varangle 25081 \quad 118-131$

$\checkmark \underline{25082} 118-131$

$\checkmark \underline{25083} \quad 118-131$
Observed Mr (expt) Mr (calc) $\begin{array}{llll}641.0033 & 1919.9880 & 1919.9840\end{array}$ $\begin{array}{lll}641.0046 & 1919.9920 & 1919.9840\end{array}$ $\begin{array}{lll}552.2883 & 1102.5620 & 1102.5619\end{array}$ $\begin{array}{llll}552.2885 & 1102.5624 & 1102.5619\end{array}$ $552.2885 \quad 1102.5625 \quad 1102.5619$ $\begin{array}{llll}552.2886 & 1102.5627 & 1102.5619\end{array}$ $\begin{array}{llll}552.2891 & 1102.5636 & 1102.5619\end{array}$ $\begin{array}{lll}760.8823 & 1519.7500 & 1519.7631\end{array}$ $760.8823 \quad 1519.7501 \quad 1519.7631$ $760.8831 \quad 1519.7517 \quad 1519.7631$ $\begin{array}{lll}760.8832 & 1519.7517 & 1519.7631\end{array}$ $\begin{array}{lll}760.8839 & 1519.7532 & 1519.7631\end{array}$ ppm M Score Expect Rank U Peptide

$\begin{array}{lllllll}2.07 & 0 & 99 & 1.2 e-10 & 1 & U & K\end{array}$

$4.180 \quad 93 \quad 5.6 e-10 \quad 1$

$0.170 \quad 92 \quad 6.6 e-10 \quad 1$

$0.520 \quad 92 \quad 6.5 e-10 \quad 1$

$0.550 \quad 926.5 e-10 \quad 1$

$0.810 \quad 92 \quad 6.5 e-10 \quad 1$

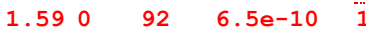

$\begin{array}{lllll}-8.620 & 62 & 6.4 e-07 \quad 1\end{array}$

$\begin{array}{lllll}-8.57 & 0 & 62 & 6.3 e-07 & 1\end{array}$

$\begin{array}{llll}-7.480 & 70 & 1.1 e-07 & 1\end{array}$

$\begin{array}{llll}-7.460 & 64 & 4 e-07 & 1\end{array}$

$-6.530 \quad 62$
U K. DGTTNIDAVAGVLFAAYLK. $S$

U K.SSVVTTAEPGR.V

U K.SSVVTTAEPGR, V

U K.SSVVTTAEPGR.V

U K.SSVVTTAEPGR.V

U K.SSVVTTAEPGR.V

U R.VVVTQDFIDASNGR. F

U R.VVVTODFIDASNGR. F

U R.VVVTQDFIDASNGR. F

U R. VVVTQDFIDASNGR. F

U R.VVVTQDFIDASNGR. F

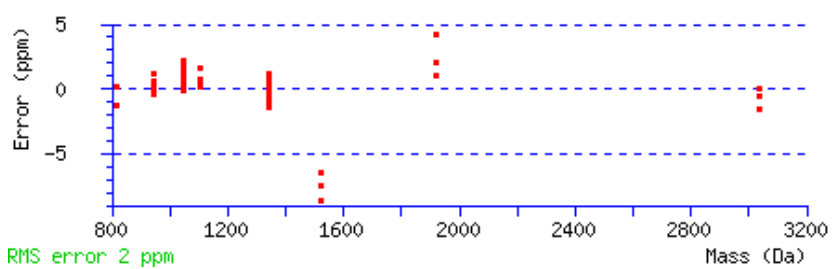

Mascot: http://www.matrixscience.com/

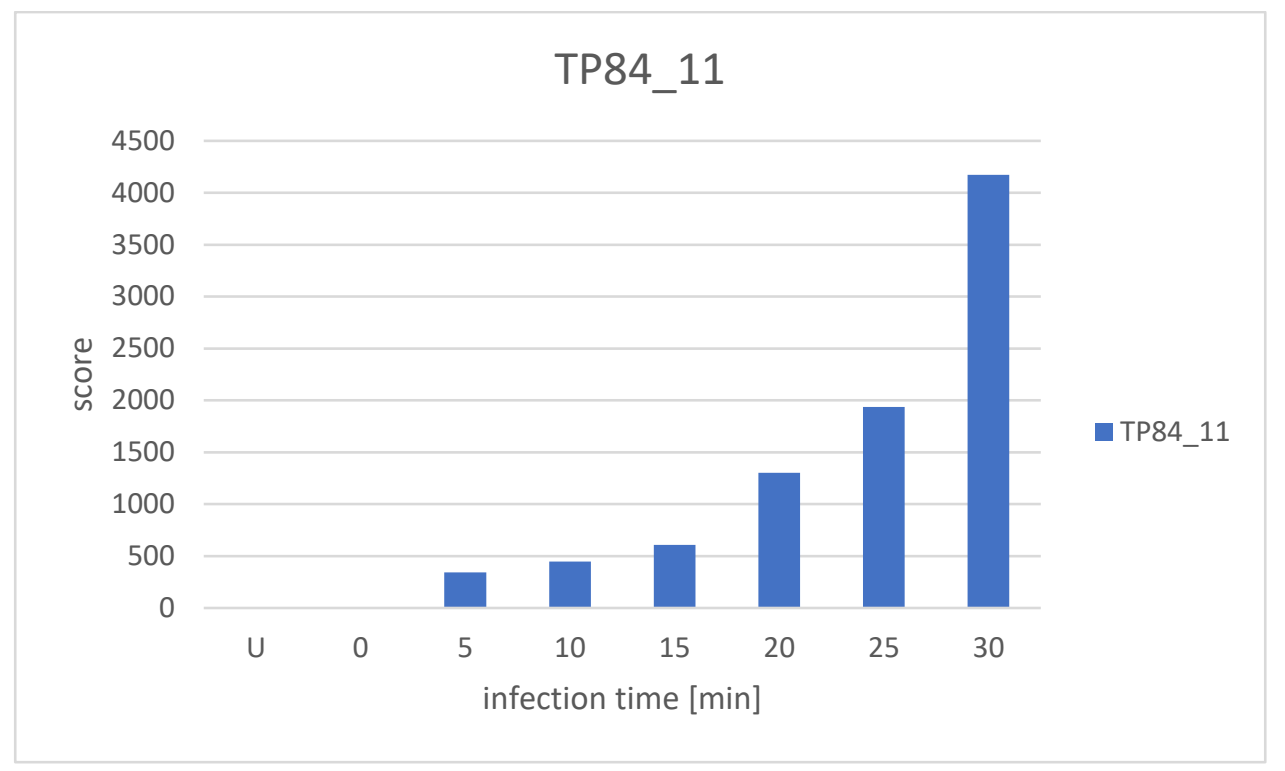




\title{
MASCOT Search Results
}

\section{Protein View: TP84_12}
Database:
TP84
Score:
164
Nominal mass $\left(\mathbf{M}_{\mathbf{r}}\right)$ : 37757
Calculated pI: $\quad 4.98$

Sequence similarity is available as an NCBI BLAST search of 67. against nr.

\section{Search parameters}

\author{
MS data file: $\quad 0: \backslash F A \backslash 02-1$ uty2018\80202240 zeb czas 0. raw \\ Enzyme: $\quad$ Trypsin: cuts C-term side of KR unless next residue is $P$. \\ Fixed modifications: $\quad$ Carbamidomethyl (C) \\ Variable modifications: Oxidation (M)
}

\section{Protein sequence coverage: $16 \%$}

Matched peptides shown in bold red.
1 MPLHLEQFQR EAFQGYVENV PPKREYALAK FMPNQPVYDI EFTYNIINGG
51 YGQMASITAW DSGAPLRDKD VIQRLTAQIA KVQHAYRLTE KELLMFHRPR
101 MDEEQQQVIQ AIYNNTDKLV WGVQDREEWL RAKAVYVGQL QYSENDVQLN
151 IDFLIPAENK LTADVDWSDP TAPVIQHLQS AVQRFKEANN GEKPVEMHMS
201 SRVETWLLQN EQVKAHIYGN TTDPRIVTSE QLQQLFSALS LPPYRVIDEQ
251 VIGENGAEAL MPEDRVVLLG EELGHTMEGP TVENNYKPGI YVIPEIKETN
301 PPROEVYVGK SVFPALERPO AVVHLIVAOS

Unformatted sequence string: $\mathbf{3 3 0}$ residues (for pasting into other applications).

Sort peptides by - Residue Number $\bigcirc$ Increasing Mass $\bigcirc$ Decreasing Mass

Show predicted peptides also

\begin{tabular}{rrrrr} 
Query & Start - End & Observed & Mr (expt) & Mr (calc) \\
$\varangle \underline{31317}$ & $161-184$ & 883.1239 & 2646.3498 & 2646.3402 \\
\hline 34727 & $266-297$ & 885.4711 & 3537.8553 & 3537.8429
\end{tabular}

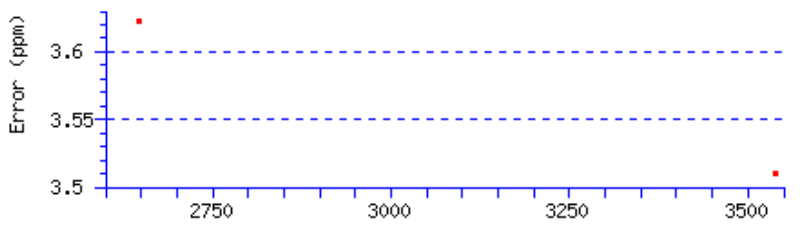

RIS error $3 \mathrm{ppm}$ Mass (Da) 


\title{
MASCOT Search Results
}

\section{Protein View: TP84_12}

\author{
Database: TP84 \\ Score: $\quad 181$ \\ Nominal mass $\left(\mathbf{M}_{\mathbf{r}}\right)$ : 37757 \\ Calculated pI: $\quad 4.98$
}

Sequence similarity is available as an NCBI BLAST search of 67. against nr.

\section{Search parameters}

\author{
MS data file: \\ 0:\FA $\backslash 02$-luty2018\80202242zeb czas10.raw \\ Enzyme: \\ Trypsin: cuts C-term side of KR unless next residue is $\mathrm{P}$. \\ Fixed modifications: Carbamidomethyl (C) \\ Variable modifications: Oxidation (M)
}

\section{Protein sequence coverage: $20 \%$}

Matched peptides shown in bold red.
1 MPLHLEQFQR EAFQGYVENV PPKREYALAK FMPNQPVYDI EFTYNIINGG
51 YGQMASITAW DSGAPLRDKD VIQRLTAQIA KVQHAYRLTE KELLMFHRPR
101 MDEEQQQVIQ AIYNNTDKLV WGVQDREEWL RAKAVYVGQL QYSENDVQLN
151 IDFLIPAENK LTADVDWSDP TAPVIQHLQS AVQRFKEANN GEKPVEMHMS
201 SRVETWLLQN EQVKAHIYGN TTDPRIVTSE QLQQLFSALS LPPYRVIDEQ
251 VIGENGAEAL MPEDRVVLLG EELGHTMEGP TVENNYKPGI YVIPEIKETN
301 PPRQEVYVGK SVFPALERPQ AVVHLIVAQS

Unformatted sequence string: $\mathbf{3 3 0}$ residues (for pasting into other applications).

Sort peptides by $\odot$ Residue Number $\bigcirc$ Increasing Mass $\bigcirc$ Decreasing Mass

Show predicted peptides also

$\begin{array}{rrrrr}\text { Query } & \text { Start - End } & \text { Observed } & \text { Mr (expt) } & \text { Mr (calc) } \\ \varangle^{299771} & 161-184 & 883.1192 & 2646.3357 & 2646.3402 \\ \square \times 8994 & 215-225 & 622.8062 & 1243.5978 & 1243.5945 \\ \varangle^{\prime 32972} & 266-297 & 885.4699 & 3537.8505 & 3537.8429\end{array}$

$\begin{array}{rrrrrll}\text { Ppm } & \text { M } & \text { Score } & \text { Expect } & \text { Rank } & \text { U } & \text { Peptide } \\ -1.69 & 0 & 109 & 1.4 \mathrm{e}-11 & 1 & \ldots & \text { U. } \\ 2.64 & 0 & 28 & 0.0015 & \frac{1}{1} & \text { U } & \text { K.AHIYGNDWTDPTAPVIQHLQSAVQR. F } \\ 2.16 & 0 & 70 & 9.7 \mathrm{e}-08 & 1 & \text { U } & \text { R. VVLLGEELGHTMEGPTVENNYKPGIYVIPEIK. E }\end{array}$

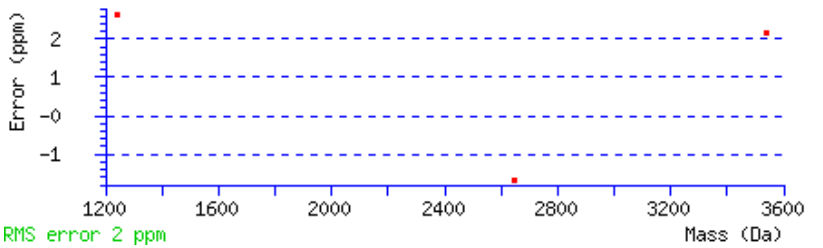




\section{MASCOT Search Results}

\section{Protein View: TP84_12}

\author{
Database: $\quad$ TP84 \\ Score: $\quad 476$ \\ Nominal mass $\left(\mathbf{M}_{\mathbf{r}}\right)$ : 37757 \\ Calculated pI: $\quad 4.98$
}

Sequence similarity is available as an NCBI BLAST search of 67. against nr.

\section{Search parameters}

\author{
MS data file: \\ $0: \backslash F A \backslash 02-l u t y 2018 \backslash 80202243 z e b$ czas15.raw \\ Enzyme: \\ Trypsin: cuts C-term side of KR unless next residue is $\mathrm{P}$. \\ Fixed modifications: Carbamidomethyl (C) \\ Variable modifications: Oxidation (M)
}

\section{Protein sequence coverage: $22 \%$}

Matched peptides shown in bold red.

1 MPLHLEQFQR EAFQGYVENV PPKREYALAK FMPNQPVYDI EFTYNIINGG

51 YGQMASITAW DSGAPLRDKD VIQRLTAQIA KVQHAYRLTE KELLMFHRPR

101 MDEEQQQVIQ AIYNNTDKLV WGVQDREEWL RAKAVYVGQL QYSENDVQLN

151 IDFLIPAENK LTADVDWSDP TAPVIQHLQS AVQRFKEANN GEKPVEMHMS

201 SRVETWLLQN EQVKAHIYGN TTDPRIVTSE QLQQLFSALS LPPYRVIDEQ

251 VIGENGAEAL MPEDRVVLLG EELGHTMEGP TVENNYKPGI YVIPEIKETN

301 PPRQEVYVGK SVFPALERPQ AVVHLIVAQS

Unformatted sequence string: $\mathbf{3 3 0}$ residues (for pasting into other applications).

Sort peptides by $\odot$ Residue Number $\bigcirc$ Increasing Mass $\bigcirc$ Decreasing Mass

Show predicted peptides also

\begin{tabular}{|c|c|c|c|c|}
\hline Query & Start - End & Observed & $\operatorname{Mr}(\exp t)$ & Mr (calc) \\
\hline 3419 & $119-126$ & 486.7736 & 971.5326 & 971.5189 \\
\hline$\varangle \underline{29561}$ & $161-184$ & 883.1193 & 2646.3360 & 2646.3402 \\
\hline$\varangle 29562$ & $161-184$ & 883.1199 & 2646.3379 & 2646.3402 \\
\hline$\square \underline{29563}$ & $161-184$ & 883.1217 & 2646.3431 & 2646.3402 \\
\hline$\triangle 8898$ & $215-225$ & 622.8043 & 1243.5941 & 1243.5945 \\
\hline$\checkmark 32877$ & $266-297$ & 885.4684 & 3537.8444 & 3537.8429 \\
\hline$\square^{\prime 32878}$ & $266-297$ & 885.4690 & 3537.8469 & 3537.8429 \\
\hline$\checkmark \underline{32879}$ & $266-297$ & 885.4692 & 3537.8476 & 3537.8429 \\
\hline$\nearrow^{\prime 32880}$ & $266-297$ & 885.4708 & 3537.8539 & 3537.8429 \\
\hline
\end{tabular}

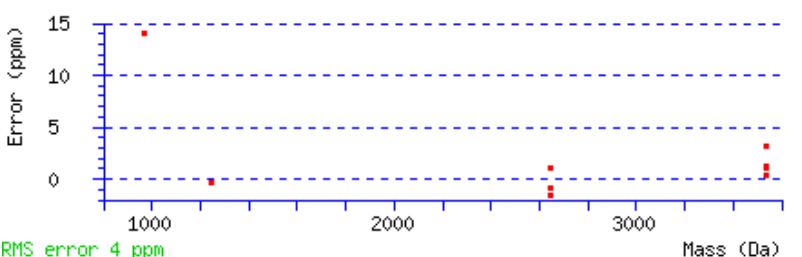

\begin{tabular}{|c|c|c|c|c|c|c|}
\hline ppm & M & Score & Expect & Rank & $\mathrm{U}$ & Peptide \\
\hline 14.1 & 0 & 14 & 0.043 & 1 & U & K. LVWGVQDR . E \\
\hline-1.58 & 0 & 108 & $1.6 e-11$ & 1 & U & K. LTADVDWSDPTAPVIQHLQSAVQR . F \\
\hline-0.86 & 0 & 122 & $6.1 e-13$ & 1 & $\mathrm{U}$ & K. LTADVDWSDPTAPVIQHLQSAVQR . F \\
\hline 1.11 & 0 & 127 & $2 e-13$ & 1 & U & K. LTADVDWSDPTAPVIQHLQSAVQR . F \\
\hline-0.38 & 0 & 28 & 0.0014 & 1 & $\mathrm{U}$ & K.AHIYGNTTDPR.I \\
\hline 0.42 & 0 & 42 & $5.7 e-05$ & 1 & U & R.VVLLGEELGHTMEGPTVENNYKPGIYVIPEIK.E \\
\hline 1.12 & 0 & 40 & 0.0001 & 1 & U & R. VVLLGEELGHTMEGPTVENNYKPGIYVIPEIK.E \\
\hline 1.33 & 0 & 55 & $3.1 e-06$ & 1 & $\mathrm{U}$ & R.VVLLGEELGHTMEGPTVENNYKPGIYVIPEIK.E \\
\hline 3.13 & 0 & 44 & $4.1 e-05$ & 1 & $\mathrm{U}$ & R. VVLLGEELGHTMEGPTVENNYKPGIYVIPEIK. E \\
\hline
\end{tabular}




\title{
MASCOT Search Results
}

\section{Protein View: TP84_12}

\author{
Database: TP84 \\ Score: $\quad 1547$ \\ Nominal mass $\left(M_{\mathbf{r}}\right): 37757$ \\ Calculated pI: $\quad 4.98$
}

Sequence similarity is available as an NCBI BLAST search of 67. against nr.

\section{Search parameters}

\author{
MS data file: $\quad 0: \backslash F A \backslash 02-1$ uty2018\80202244zeb czas20.raw \\ Enzyme: $\quad$ Trypsin: cuts C-term side of KR unless next residue is $P$. \\ Fixed modifications: Carbamidomethyl (C) \\ Variable modifications: Oxidation (M)
}

\section{Protein sequence coverage: $\mathbf{2 8 \%}$}

Matched peptides shown in bold red.

1 MPLHLEQFQR EAFQGYVENV PPKREYALAK FMPNQPVYDI EFTYNIINGG 51 YGQMASITAW DSGAPLRDKD VIQRLTAQIA KVQHAYRLTE KELLMFHRPR 101 MDEEQQQVIQ AIYNNTDKLV WGVQDREEWL RAKAVYVGQL QYSENDVQLN 151 IDFLIPAENK LTADVDWSDP TAPVIQHLQS AVQREKEANN GEKPVEMHMS 201 SRVETWLLQN EQVKAHIYGN TTDPRIVTSE QLQQLFSALS LPPYRVIDEQ 251 VIGENGAEAL MPEDRVVLLG EELGHTMEGP TVENNYKPGI YVIPEIKETN 301 PPRQEVYVGK SVFPALERPQ AVVHLIVAQS

Unformatted sequence string: $\mathbf{3 3 0}$ residues (for pasting into other applications).

Sort peptides by - Residue Number $\bigcirc$ Increasing Mass $\bigcirc$ Decreasing Mass

Show predicted peptides also

\begin{tabular}{|c|c|c|c|c|}
\hline Query & Start - End & Observed & $M r(\exp t)$ & $\operatorname{Mr}$ (calc) \\
\hline$\varangle 13816$ & $2-10$ & 389.8817 & 1166.6232 & 1166.6196 \\
\hline$\square 13817$ & $2-10$ & 389.8820 & 1166.6241 & 1166.6196 \\
\hline$\overline{13818}$ & $2-10$ & 389.8822 & 1166.6248 & 1166.6196 \\
\hline 39909 & $101-118$ & 1084.0054 & 2165.9962 & 2165.9899 \\
\hline$\checkmark 39910$ & $101-118$ & 1084.0112 & 2166.0078 & 2165.9899 \\
\hline$\overline{43995}$ & $161-184$ & 883.1211 & 2646.3415 & 2646.3402 \\
\hline$\longdiv { 4 3 9 9 6 }$ & $161-184$ & 883.1216 & 2646.3429 & 2646.3402 \\
\hline 4 & $161-184$ & 883.1216 & 2646.3429 & 2646.3402 \\
\hline$\triangle 43998$ & $161-184$ & 883.1217 & 2646.3434 & 2646.3402 \\
\hline$\longdiv { 4 3 9 9 9 }$ & $161-184$ & 883.1223 & 2646.3452 & 2646.3402 \\
\hline$\checkmark 4$ & $161-184$ & 883.1225 & 2646.3456 & 2646.3402 \\
\hline$\checkmark 44001$ & $161-184$ & 883.1225 & 2646.3457 & 2646.3402 \\
\hline$\square \underline{44002}$ & $161-184$ & 883.1230 & 2646.3471 & 2646.3402 \\
\hline 444003 & $161-184$ & 883.1241 & 2646.3504 & 2646.3402 \\
\hline$\triangle 16856$ & $215-225$ & 622.8080 & 1243.6013 & 1243.5945 \\
\hline 447347 & $266-297$ & 885.4690 & 3537.8470 & 3537.8429 \\
\hline$\triangle \underline{47348}$ & $266-297$ & 885.4695 & 3537.8490 & 3537.8429 \\
\hline 44749 & $266-297$ & 885.4699 & 3537.8506 & 3537.8429 \\
\hline 447350 & $266-297$ & 1180.2910 & 3537.8512 & 3537.8429 \\
\hline$\overline{47351}$ & $266-297$ & 885.4710 & 3537.8549 & 3537.8429 \\
\hline
\end{tabular}

\begin{tabular}{|c|c|c|c|c|c|c|}
\hline ppm & M & Score & Expect & Rank & $\mathrm{U}$ & Peptide \\
\hline 3.07 & 0 & 58 & $1.7 e-06$ & 1 & $\mathrm{U}$ & M. PLHLEQFQR. E \\
\hline .79 & 0 & 58 & $1.7 e-06$ & 1 & $\mathrm{U}$ & M. PLHLEQFQR.E \\
\hline .43 & 0 & 58 & $1.7 e-06$ & 1 & $\mathrm{U}$ & M. PLHLEQFQR. E \\
\hline .94 & 0 & 108 & $1.5 e-11$ & 1 & $\mathrm{U}$ & R. MDEEQQQVIQAIYNNTDK. L \\
\hline 8.29 & 0 & 116 & $2.4 e-12$ & 1 & $\mathrm{U}$ & R. MDEEQQQVIQAIYNNTDK. L \\
\hline .49 & 0 & 111 & $7.2 e-12$ & 1 & $\mathrm{U}$ & K. LTADVDWSDPTAPVIQHLQSAVQR . F \\
\hline 1.02 & 0 & 83 & $5.3 e-09$ & 1 & $\mathrm{U}$ & K. LTADVDWSDPTAPVIQHLQSAVQR . F \\
\hline 1.03 & 0 & 124 & $4.4 e-13$ & 1 & $\mathrm{U}$ & K. LTADVDWSDPTAPVIQHLQSAVQR . F \\
\hline 1.21 & 0 & 132 & $6.9 e-14$ & 1 & $\mathrm{U}$ & K. LTADVDWSDPTAPVIQHLQSAVQR . F \\
\hline 1.89 & 0 & 93 & $4.9 e-10$ & 1 & $\mathrm{U}$ & K. LTADVDWSDPTAPVIQHLQSAVQR . F \\
\hline .02 & 0 & 135 & $3.3 e-14$ & 1 & $\mathrm{U}$ & K. LTADVDWSDPTAPVIQHLQSAVQR . F \\
\hline .08 & 0 & 137 & $2.2 e-14$ & 1 & $\mathrm{U}$ & K. LTADVDWSDPTAPVIQHLQSAVQR . F \\
\hline 2.59 & 0 & 142 & $6.2 e-15$ & 1 & $\mathrm{U}$ & K. LTADVDWSDPTAPVIQHLQSAVQR . F \\
\hline 3.85 & 0 & 58 & $1.4 e-06$ & 1 & $\mathrm{U}$ & K. LTADVDWSDPTAPVIQHLQSAVQR . F \\
\hline .47 & 0 & 65 & $3.4 e-07$ & 1 & $\mathrm{U}$ & K. AHIYGNTTDPR. I \\
\hline 1.17 & 0 & 60 & $1 e-06$ & 1 & $\mathrm{U}$ & R. VVLLGEELGHTMEGPTVENNYKPGIYVIPEIK. E \\
\hline 1.72 & 0 & 61 & $7.4 e-07$ & 1 & $\mathrm{U}$ & R. VVLLGEELGHTMEGPTVENNYKPGIYVIPEIK. E \\
\hline 18 & 0 & 57 & $1.8 e-06$ & 1 & $\mathrm{U}$ & R. VVLLGEELGHTMEGPTVENNYKPGIYVIPEIK. E \\
\hline & & 81 & $8 e-09$ & 1 & $\mathrm{U}$ & R. VVLLGEELGHTMEGPTVENNYKPGIYVIPEIK. E \\
\hline & & 58 & $7 e-06$ & 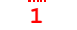 & & \\
\hline
\end{tabular}

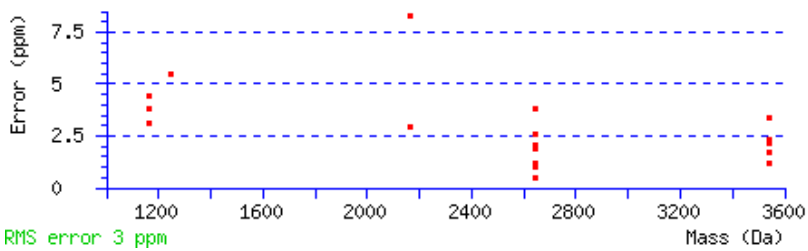




\title{
MASCOT Search Results
}

\section{Protein View: TP84_12}

\author{
Database: $\quad$ TP84 \\ Score: $\quad 2724$ \\ Nominal mass $\left(\mathbf{M}_{\mathbf{r}}\right): 37757$ \\ Calculated pI: $\quad 4.98$
}

Sequence similarity is available as an NCBI BLAST search of 67. against nr.

\section{Search parameters}

MS data file: $\quad 0: \backslash F A \backslash 02-1$ uty2018\80202245zeb czas25.raw

Enzyme: $\quad$ Trypsin: cuts C-term side of KR unless next residue is $P$.

Fixed modifications: $\quad$ Carbamidomethyl (C)

Variable modifications: Oxidation (M)

\section{Protein sequence coverage: $49 \%$}

Matched peptides shown in bold red.

1 MPLHLEQFQR EAFQGYVENV PPKREYALAK FMPNQPVYDI EFTYNIINGG

51 YGQMASITAW DSGAPLRDKD VIQRLTAQIA KVQHAYRLTE KELLMFHRPR 101 MDEEQQQVIQ AIYNNTDKLV WGVQDREEWL RAKAVYVGQL QYSENDVQLN 151 IDFLIPAENK LTADVDWSDP TAPVIQHLQS AVQRFKEANN GEKPVEMHMS 201 SRVETWLLQN EQVKAHIYGN TTDPRIVTSE QLQQLFSALS LPPYRVIDEQ 251 VIGENGAEAL MPEDRVVLLG EELGHTMEGP TVENNYKPGI YVIPEIKETN 301 PPRQEVYVGK SVFPALERPQ AVVHLIVAQS

Unformatted sequence string: $\mathbf{3 3 0}$ residues (for pasting into other applications).

Sort peptides by - Residue Number $\bigcirc$ Increasing Mass $\bigcirc$ Decreasing Mass

Show predicted peptides also

\begin{tabular}{|c|c|}
\hline Query & Start - End \\
\hline$\checkmark 8703$ & $2-10$ \\
\hline$\varangle \underline{8704}$ & $2-10$ \\
\hline 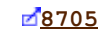 & $2-10$ \\
\hline 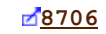 & $2-10$ \\
\hline$\overleftarrow{8707}$ & $2-10$ \\
\hline$\overleftarrow{8708}$ & $2-10$ \\
\hline$\square^{\prime} 16970$ & $11-23$ \\
\hline$\square^{16973}$ & $11-23$ \\
\hline$\square^{\prime 16977}$ & $11-23$ \\
\hline$\checkmark 16978$ & $11-23$ \\
\hline$\checkmark 30492$ & $101-118$ \\
\hline$\checkmark 30493$ & $101-118$ \\
\hline$\widetilde{30494}$ & $101-118$ \\
\hline$\sigma^{3} 30495$ & $101-118$ \\
\hline$\checkmark 34495$ & $161-184$ \\
\hline$\checkmark 34496$ & $161-184$ \\
\hline$\checkmark 34497$ & $161-184$ \\
\hline$\nabla 34498$ & $161-184$ \\
\hline$\checkmark 34499$ & $161-184$ \\
\hline$\varangle \underline{25060}$ & $187-202$ \\
\hline$\square^{\prime 17287}$ & $203-214$ \\
\hline$\triangle 17288$ & $203-214$ \\
\hline$\triangle^{\prime 17289}$ & $203-214$ \\
\hline$\varangle 17290$ & $203-214$ \\
\hline$\varpi^{\prime 17291}$ & $203-214$ \\
\hline$\triangle 17292$ & $203-214$ \\
\hline $\mathbb{\nearrow}^{\prime 17293}$ & $203-214$ \\
\hline$\checkmark 10832$ & $215-225$ \\
\hline$\triangle 10833$ & $215-225$ \\
\hline$\checkmark 37983$ & $266-297$ \\
\hline$\underbrace{\prime 37984}$ & $266-297$ \\
\hline$\checkmark \underline{37985}$ & $266-297$ \\
\hline$\triangle \underline{37986}$ & $266-297$ \\
\hline$\checkmark 37987$ & $266-297$ \\
\hline$\underline{37988}$ & $266-297$ \\
\hline$\checkmark 37989$ & $266-297$ \\
\hline$\checkmark \underline{37990}$ & $266-297$ \\
\hline$\triangle 1460$ & $304-310$ \\
\hline 730437 & $311-330$ \\
\hline
\end{tabular}

$\begin{array}{rrr}\text { Observed } & \text { Mr (expt) } & \text { Mr (calc) } \\ 389.8800 & 1166.6182 & 1166.6196 \\ 389.8802 & 1166.6187 & 1166.6196 \\ 389.8802 & 1166.6189 & 1166.6196 \\ 389.8805 & 1166.6196 & 1166.6196 \\ 584.3181 & 1166.6217 & 1166.6196 \\ 584.3182 & 1166.6219 & 1166.6196 \\ 739.3652 & 1476.7159 & 1476.7249 \\ 739.3667 & 1476.7187 & 1476.7249 \\ 739.3706 & 1476.7266 & 1476.7249 \\ 739.3706 & 1476.7267 & 1476.7249 \\ 1083.9981 & 2165.9816 & 2165.9899 \\ 1083.9984 & 2165.9822 & 2165.9899 \\ 723.0019 & 2165.9840 & 2165.9899 \\ 1083.9997 & 2165.9848 & 2165.9899 \\ 883.1196 & 2646.3369 & 2646.3402 \\ 883.1196 & 2646.3371 & 2646.3402 \\ 883.1200 & 2646.3382 & 2646.3402 \\ 883.1209 & 2646.3408 & 2646.3402 \\ 883.1213 & 2646.3421 & 2646.3402 \\ 605.9417 & 1814.8032 & 1814.8039 \\ 743.8986 & 1485.7827 & 1485.7827 \\ 743.8990 & 1485.7834 & 1485.7827 \\ 743.8991 & 1485.7836 & 1485.7827 \\ 743.8991 & 1485.7837 & 1485.7827 \\ 743.8995 & 1485.7844 & 1485.7827 \\ 743.8996 & 1485.7847 & 1485.7827 \\ 743.8997 & 1485.7849 & 1485.7827 \\ 622.8047 & 1243.5947 & 1243.5945 \\ 622.8050 & 1243.5954 & 1243.5945 \\ 1180.2857 & 3537.8353 & 3537.8429 \\ 1180.2857 & 3537.8353 & 3537.8429 \\ 885.4665 & 3537.8367 & 3537.8429 \\ 1180.2865 & 3537.8377 & 3537.8429 \\ 885.4669 & 3537.8386 & 3537.8429 \\ 885.4672 & 3537.8396 & 3537.8429 \\ 708.5755 & 3537.8410 & 3537.8429 \\ 885.4680 & 3537.8428 & 3537.8429 \\ 411.7213 & 821.4281 & 821.4283 \\ 1081.1114 & 2160.2082 & 2160.2055\end{array}$

ppm M Score Expect Rank U Peptide

$\begin{array}{lllll}-1.20 & 0 & 58 & 1.7 e-06 & 1\end{array}$

$-0.810 \quad 57$

$-0.630$

$-0.0150$

1.750

1.950

$-6.050$

$-4.160$

1.170

1.210

$-3.800$

$-3.520$

$-2.710$

$-2.320$

$-1.260$

$-1.160$

$-0.740$

0.240

0.730

$-0.410$

$-0.00130$

0.460

0.600

0.630

1.160

1. 320

1.450

0.160

0.700

$-2.150$

$-2.150$

$-1.740$

$-1.470$

$-1.210$

$-0.930$

$-0.540$

$-0.0290$

$-0.250$

1.260
$1.9 e-06 \quad 1$

$1.7 e-06$

1. $8 e-06$

7.2e-07

1. $9 e-06$

5. $5 e-06$

$5.9 \mathrm{e}-08$

$1.8 e-07$

1. $7 e-07$

$2 e-12$

3. $1 \mathrm{e}-12$

2. $6 \mathrm{e}-06$

1. $4 \mathrm{e}-13$

$2.5 e-14$

$5.6 e-15$

1. $2 \mathrm{e}-14$

$7.8 e-16$

$1 e-14$

3. $4 \mathrm{e}-09$

1. $3 e-07$

3. $1 e-07$

$3 e-07$

2. $9 e-08$

6. $3 e-07$

3. $7 e-07$

$7.8 e-07$

3. $6 e-07$

$1 e-06$

3. $5 e-11$

2. $9 e-14$

2. $7 e-07$

$6.5 e-12$

1. $6 \mathrm{e}-06$

$3.2 e-07$

$8.1 \mathrm{e}-07$

$7.2 \mathrm{e}-08$

$8.1 e-07$

$1.2 e-08$
U M. PLHLEQFQR.

U M. PLHLEOFOR.E

U M. PLHLEQFQR.E

U M. PLHLEQFQR.E

U M. PLHLEQFOR . E

U M. PLHLEQFQR.E

U R. EAFQGYVENVPPK.R

U R.EAFQGYVENVPPK.R

U R.EAFQGYVENVPPK.R

U R. EAFQGYVENVPPK.R

U R.MDEEQOOVIOAIYNNTDK. L

U R.MDEEQQQVIQAIYNNTDK.I

U R.MDEEQQQVIQAIYNNTDK. L

U R.MDEEQQQVIOAIYNNTDK. I

U K. LTADVDWSDPTAPVIQHLQSAVQR.F

U K. LTADVDWSDPTAPVIQHLQSAVQR. F

K. LTADVDWSDPTAPVIOHLOSAVOR.F

U K. LTADVDWSDPTAPVIQHLQSAVQR. F

U K. LTADVDWSDPTAPVIQHLQSAVQR . F

$U$ U E.ANNGEKPVEMHMSSR $V$

U R.VETWLLQNEQVK. A

U R.VETWLLQNEQVK. A

U R.VETWLLONEOVK. A

U R. VETWLLQNEQVK. A

U R. VETWLLQNEQVK. A

U R. VETWLLONEOVK. A

U R. VETWLLQNEQVK. A

U K. AHIYGNTTDPR. I

U K.AHIYGNTTDPR.I

U R.VVLLGEELGHTMEGPTVENNYKPGIYVIPEIK.E

U R.VVLLGEELGHTMEGPTVENNYKPGIYVIPEIK . E

U R.VVLLGEELGHTMEGPTVENNYKPGIYVIPEIK.E

U R.VVLLGEELGHTMEGPTVENNYKPGIYVIPEIK.E

U R.VVLLGEELGHTMEGPTVENNYKPGIYVIPEIK. E

U R.VVLLGEELGHTMEGPTVENNYKPGIYVIPEIK.E

U R. VVLLGEELGHTMEGPTVENNYKPGIYVIPEIK.E

U R.VVLLGEELGHTMEGPTVENNYKPGIYVIPEIK.E

U R. QEVYVGK.S

U K. SVFPALERPOAVVHLIVAOS.- 


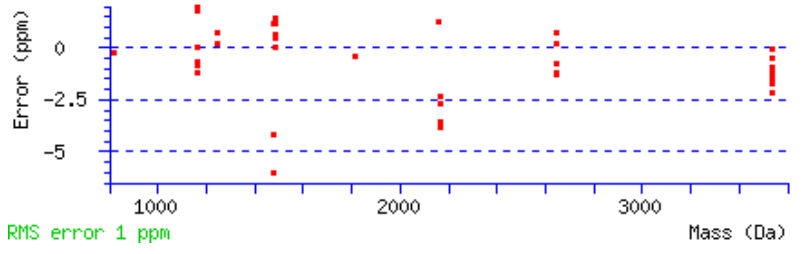




\title{
MASCOT Search Results
}

\section{Protein View: TP84_12}

\author{
Database: TP84 \\ Score: $\quad 5774$ \\ Nominal mass $\left(M_{\mathbf{r}}\right): 37757$ \\ Calculated pI: $\quad 4.98$
}

Sequence similarity is available as an NCBI BLAST search of 67. against nr.

\section{Search parameters}

MS data file: $\quad 0: \backslash F A \backslash 02-1$ uty2018\80202246zeb czas30.raw

Enzyme: $\quad$ Trypsin: cuts C-term side of KR unless next residue is $P$.

Fixed modifications: $\quad$ Carbamidomethyl (C)

Variable modifications: Oxidation (M)

\section{Protein sequence coverage: $\mathbf{5 7 \%}$}

Matched peptides shown in bold red.

1 MPLHLEQFQR EAFQGYVENV PPKREYALAK FMPNQPVYDI EFTYNIINGG

51 YGQMASITAW DSGAPLRDKD VIQRLTAQIA KVQHAYRLTE KELLMFHRPR 101 MDEEQQQVIQ AIYNNTDKLV WGVQDREEWL RAKAVYVGQL QYSENDVQLN 151 IDFLIPAENK LTADVDWSDP TAPVIQHLQS AVQRFKEANN GEKPVEMHMS 201 SRVETWLIQN EQVKAHIYGN TTDPRIVTSE QLQQLFSALS LPPYRVIDEQ 251 VIGENGAEAL MPEDRVVLIG EELGHTMEGP TVENNYKPGI YVIPEIKETN 301 PPRQEVYVGK SVFPALERPQ AVVHLIVAQS

Unformatted sequence string: $\mathbf{3 3 0}$ residues (for pasting into other applications).

Sort peptides by - Residue Number $\bigcirc$ Increasing Mass $\bigcirc$ Decreasing Mass

Show predicted peptides also

\begin{tabular}{|c|c|}
\hline Query & Start - End \\
\hline$\checkmark 12089$ & $2-10$ \\
\hline$\triangle 12090$ & $2-10$ \\
\hline$\checkmark 12091$ & $2-10$ \\
\hline$\checkmark 12092$ & $2-10$ \\
\hline$\nearrow^{\prime} 12093$ & $2-10$ \\
\hline$\overline{12094}$ & $2-10$ \\
\hline$\checkmark 12095$ & $2-10$ \\
\hline$\overline{12096}$ & $2-10$ \\
\hline$\checkmark 12097$ & $2-10$ \\
\hline$\overline{12098}$ & $2-10$ \\
\hline$\checkmark 12099$ & $2-10$ \\
\hline$\overline{12100}$ & $2-10$ \\
\hline$\square 23758$ & $11-23$ \\
\hline$\overline{23759}$ & $11-23$ \\
\hline$\triangle 23760$ & $11-23$ \\
\hline$\longdiv { 3 9 7 9 5 }$ & $101-118$ \\
\hline$\checkmark \underline{39796}$ & $101-118$ \\
\hline$\overline{39797}$ & $101-118$ \\
\hline 39798 & $101-118$ \\
\hline$\checkmark \overline{39799}$ & $101-118$ \\
\hline$\overline{39800}$ & $101-118$ \\
\hline$\sqrt{39802}$ & $101-118$ \\
\hline$\checkmark 5436$ & $119-126$ \\
\hline$\longdiv { 5 4 3 7 }$ & $119-126$ \\
\hline$\longdiv { 5 4 3 8 }$ & $119-126$ \\
\hline$\checkmark 5439$ & $119-126$ \\
\hline$\longdiv { 5 4 4 2 }$ & $119-126$ \\
\hline$\overline{5443}$ & $119-126$ \\
\hline$\checkmark 5444$ & $119-126$ \\
\hline$\checkmark 4 \overline{4348}$ & $161-184$ \\
\hline 444349 & $161-184$ \\
\hline$\triangle 44350$ & $161-184$ \\
\hline$\square 44351$ & $161-184$ \\
\hline$\longdiv { 4 4 3 5 2 }$ & $161-184$ \\
\hline 444353 & $161-184$ \\
\hline$\checkmark \overline{44354}$ & $161-184$ \\
\hline$\longdiv { 4 4 3 5 5 }$ & $161-184$ \\
\hline$\square \underline{44356}$ & $161-184$ \\
\hline$\triangle 44357$ & $161-184$ \\
\hline$\triangle 44358$ & $161-184$ \\
\hline 33728 & $187-202$ \\
\hline$\triangle^{3} 33730$ & $187-202$ \\
\hline$\square 24064$ & $203-214$ \\
\hline$\checkmark 24065$ & $203-214$ \\
\hline$\checkmark 24066$ & $203-214$ \\
\hline
\end{tabular}

$\begin{array}{rrr}\text { Observed } & \text { Mr (expt) } & \text { Mr (calc) } \\ 389.8793 & 1166.6162 & 1166.6196 \\ 389.8795 & 1166.6168 & 1166.6196 \\ 389.8797 & 1166.6173 & 1166.6196 \\ 389.8798 & 1166.6176 & 1166.6196 \\ 389.8799 & 1166.6178 & 1166.6196 \\ 584.3163 & 1166.6181 & 1166.6196 \\ 389.8800 & 1166.6181 & 1166.6196 \\ 389.8801 & 1166.6184 & 1166.6196 \\ 389.8801 & 1166.6184 & 1166.6196 \\ 584.3165 & 1166.6185 & 1166.6196 \\ 584.3171 & 1166.6197 & 1166.6196 \\ 584.3176 & 1166.6207 & 1166.6196 \\ 739.3701 & 1476.7256 & 1476.7249 \\ 739.3732 & 1476.7318 & 1476.7249 \\ 739.3734 & 1476.7323 & 1476.7249 \\ 1083.9988 & 2165.9830 & 2165.9899 \\ 723.0017 & 2165.9831 & 2165.9899 \\ 1083.9996 & 2165.9846 & 2165.9899 \\ 1083.9997 & 2165.9848 & 2165.9899 \\ 723.0025 & 2165.9858 & 2165.9899 \\ 723.0030 & 2165.9871 & 2165.9899 \\ 723.0042 & 2165.9907 & 2165.9899 \\ 486.7665 & 971.5185 & 971.5189 \\ 486.7667 & 971.5188 & 971.5189 \\ 486.7668 & 971.5190 & 971.5189 \\ 486.7668 & 971.5190 & 971.5189 \\ 486.7668 & 971.5191 & 971.5189 \\ 486.7669 & 971.5193 & 971.5189 \\ 486.7669 & 971.5193 & 971.5189 \\ 883.1194 & 2646.3362 & 2646.3402 \\ 883.1195 & 2646.3366 & 2646.3402 \\ 883.1196 & 2646.3369 & 2646.3402 \\ 883.1198 & 2646.3375 & 2646.3402 \\ 883.1200 & 2646.3381 & 2646.3402 \\ 883.1200 & 2646.3383 & 2646.3402 \\ 883.1202 & 2646.3387 & 2646.3402 \\ 883.1204 & 2646.3392 & 2646.3402 \\ 883.1205 & 2646.3395 & 2646.3402 \\ 883.1205 & 2646.3396 & 2646.3402 \\ 883.1207 & 2646.3402 & 2646.3402 \\ 605.9417 & 1814.8033 & 1814.8039 \\ 605.9424 & 1814.8053 & 1814.8039 \\ 743.8977 & 1485.7808 & 1485.7827 \\ 743.8980 & 1485.7814 & 1485.7827 \\ 743.8981 & 1485.7816 & 1485.7827\end{array}$

U Peptide

Ü M. PLHLEQFQR.E

U M. PLHLEOFOR.E

U M. PLHLEQFQR.E

U M. PLHLEQFQR.E

U M. PLHLEQFOR.E

U M. PLHLEQFQR.E

U M. PLHLEQFQR.E

U M. PLHLEOFOR.E

U M. PLHLEQFQR.E

U M. PLHLEQFQR.E

U M. PLHLEQFQR.E

U M. PLHLEQFQR.E

U R.EAFQGYVENVPPK.R

U R. EAFOGYVENVPPK.R

U R. EAFQGYVENVPPK.R

U R.MDEEQQQVIQAIYNNTDK. L

U R.MDEEQQQVIQAIYNNTDK. I

U R.MDEEQQQVIQAIYNNTDK. L

U R.MDEEQQQVIQAIYNNTDK. L

U R.MDEEQQQVIQAIYNNTDK. L

U R.MDEEQQQVIQAIYNNTDK. L

U R.MDEEOOOVIOAIYNNTDK. I

U K. LVWGVQDR.E

U K. LVWGVQDR.E

U K. LVWGVQDR.E

U K. LVWGVODR. F

U K. LVWGVQDR.E

U K. LVWGVQDR.E

U K. LVWGVQDR.E

U K. LTADVDWSDPTAPVIQHLQSAVQR.F

U K. LTADVDWSDPTAPVIOHLQSAVQR . F

U K. LTADVDWSDPTAPVIQHLQSAVQR.F

U K. LTADVDWSDPTAPVIQHLQSAVQR. F

U K. LTADVDWSDPTAPVIOHLOSAVOR . F

U K. LTADVDWSDPTAPVIQHLQSAVQR. F

U K. LTADVDWSDPTAPVIQHLQSAVQR.F

U K. LTADVDWSDPTAPVIOHLOSAVQR . F

U K. LTADVDWSDPTAPVIQHLQSAVQR. F

U K. LTADVDWSDPTAPVIQHLQSAVQR . F

U K. LTADVDWSDPTAPVIOHLOSAVOR F

U K. EANNGEKPVEMHMSSR.V

U K. EANNGEKPVEMHMSSR.V

U R. VETWLLQNEOVK. A

U R.VETWLLQNEQVK. A

U R. VETWLLONEOVK A 
Mascot Search Results: 67.

\begin{tabular}{|c|c|}
\hline & \\
\hline & $203-$ \\
\hline & $203-21$ \\
\hline & $03-2$ \\
\hline 40 & $203-21$ \\
\hline 40 & $203-214$ \\
\hline 240 & $03-21$ \\
\hline 40 & $203-214$ \\
\hline & $03-$ \\
\hline 40 & $203-214$ \\
\hline 40 & $203-214$ \\
\hline$=30$ & $03-21$ \\
\hline 40 & $203-214$ \\
\hline $4 c^{2}>>$ & $203-214$ \\
\hline$\underline{0}$ & $203-214$ \\
\hline 40 & $203-214$ \\
\hline $40 \mathrm{C}$ & $03-214$ \\
\hline & $03-$ \\
\hline 40 & $03-214$ \\
\hline- & $15-225$ \\
\hline 51 & $215-225$ \\
\hline 51 & $15-225$ \\
\hline & 225 \\
\hline 51 & $215-225$ \\
\hline 51 & $215-225$ \\
\hline & $215-225$ \\
\hline & $15-225$ \\
\hline 99 & $246-265$ \\
\hline $20+2>$ & $66-297$ \\
\hline 18 & $266-297$ \\
\hline 82 & $266-297$ \\
\hline 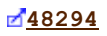 & $266-297$ \\
\hline 82 & $266-297$ \\
\hline & $266-297$ \\
\hline & $266-297$ \\
\hline 182 & $266-297$ \\
\hline & $266-297$ \\
\hline & $266-297$ \\
\hline & $266-297$ \\
\hline & 297 \\
\hline & $304-31$ \\
\hline & \\
\hline
\end{tabular}

Observed Mr (expt) Mr (calc) $\begin{array}{lll}743.8984 & 1485.7822 & 1485.7827\end{array}$ $\begin{array}{lll}743.8984 & 1485.7822 & 1485.7827\end{array}$ $\begin{array}{lll}743.8985 & 1485.7824 & 1485.7827\end{array}$ $\begin{array}{lll}743.8985 & 1485.7824 & 1485.7827\end{array}$ $\begin{array}{lll}743.8986 & 1485.7826 & 1485.7827\end{array}$ $\begin{array}{lll}743.8986 & 1485.7826 & 1485.7827\end{array}$ $\begin{array}{lll}743.8986 & 1485.7827 & 1485.7827\end{array}$ $\begin{array}{lll}743.8987 & 1485.7829 & 1485.7827\end{array}$ $\begin{array}{lll}743.8987 & 1485.7829 & 1485.7827\end{array}$ $\begin{array}{lll}743.8987 & 1485.7829 & 1485.7827\end{array}$ $\begin{array}{lll}743.8989 & 1485.7833 & 1485.7827\end{array}$ $\begin{array}{lll}743.8991 & 1485.7836 & 1485.7827\end{array}$ $\begin{array}{llll}743.8991 & 1485.7836 & 1485.7827\end{array}$ $\begin{array}{lll}743.8992 & 1485.7839 & 1485.7827\end{array}$ $\begin{array}{lll}743.8994 & 1485.7842 & 1485.7827\end{array}$ $\begin{array}{llll}743.8997 & 1485.7848 & 1485.7827\end{array}$ $\begin{array}{lll}743.8999 & 1485.7852 & 1485.7827\end{array}$ $\begin{array}{llll}743.9006 & 1485.7866 & 1485.7827\end{array}$ $\begin{array}{lll}415.5381 & 1243.5925 & 1243.5945\end{array}$ $\begin{array}{llll}415.5383 & 1243.5930 & 1243.5945\end{array}$ $\begin{array}{llll}415.5384 & 1243.5933 & 1243.5945\end{array}$ $\begin{array}{llll}415.5385 & 1243.5937 & 1243.5945\end{array}$ $\begin{array}{llll}622.8041 & 1243.5937 & 1243.5945\end{array}$ $622.8043 \quad 1243.5940 \quad 1243.5945$ $\begin{array}{lll}622.8044 & 1243.5943 & 1243.5945\end{array}$ $\begin{array}{llll}622.8047 & 1243.5949 & 1243.5945\end{array}$ $\begin{array}{llll}729.0181 & 2184.0324 & 2184.0368\end{array}$ $\begin{array}{lll}1180.2864 & 3537.8374 & 3537.8429\end{array}$ $\begin{array}{llll}1180.2865 & 3537.8377 & 3537.8429\end{array}$ $\begin{array}{lll}885.4669 & 3537.8383 & 3537.8429\end{array}$ $\begin{array}{llll}1180.2876 & 3537.8410 & 3537.8429\end{array}$ $\begin{array}{lll}885.4678 & 3537.8420 & 3537.8429\end{array}$ $\begin{array}{llll}1180.2880 & 3537.8422 & 3537.8429\end{array}$ $\begin{array}{lll}885.4679 & 3537.8425 & 3537.8429\end{array}$ $\begin{array}{lll}1180.2881 & 3537.8425 & 3537.8429\end{array}$ $\begin{array}{llll}1180.2882 & 3537.8428 & 3537.8429\end{array}$ $\begin{array}{lll}708.5760 & 3537.8435 & 3537.8429\end{array}$ $\begin{array}{llll}885.4693 & 3537.8483 & 3537.8429\end{array}$ $\begin{array}{llll}708.5779 & 3537.8529 & 3537.8429\end{array}$ $\begin{array}{lll}411.7212 & 821.4279 & 821.4283\end{array}$ $\begin{array}{lll}1081.1081 & 2160.2016 \quad 2160.2055\end{array}$

\begin{tabular}{|c|c|c|c|c|}
\hline ppm & M & Score & Expect & Ran \\
\hline-0.34 & 0 & 82 & $6.2 e-09$ & \\
\hline-0.32 & 0 & 83 & $5.2 e-09$ & \\
\hline-0.22 & 0 & 73 & $5.6 e-08$ & \\
\hline-0.19 & 0 & 53 & $4.8 e-06$ & \\
\hline-0.096 & 0 & 77 & $1.9 e-08$ & \\
\hline-0.055 & 0 & 84 & $3.8 e-09$ & \\
\hline 0.042 & 0 & 50 & $9.2 e-06$ & \\
\hline 0.093 & 0 & 80 & $1.1 e-08$ & \\
\hline 0.13 & 0 & 71 & $8.2 e-08$ & \\
\hline 0.15 & 0 & 65 & $3.1 e-07$ & \\
\hline 0.36 & 0 & 77 & $2 e-08$ & \\
\hline 0.58 & 0 & 82 & $6.7 e-09$ & \\
\hline 0.60 & 0 & 64 & $4.1 e-07$ & \\
\hline 0.81 & 0 & 52 & $6.1 e-06$ & \\
\hline 0.97 & 0 & 59 & $1.4 e-06$ & \\
\hline 1.39 & 0 & 59 & $1.2 \mathrm{e}-06$ & \\
\hline 1.65 & 0 & 76 & $2.5 e-08$ & \\
\hline 2.60 & 0 & 76 & $2.6 e-08$ & \\
\hline-1.67 & 0 & 53 & $5.4 e-06$ & \\
\hline-1.28 & 0 & 67 & $2.1 e-07$ & \\
\hline-1.01 & 0 & 60 & $1.1 e-06$ & \\
\hline-0.70 & 0 & 63 & $4.8 e-07$ & \\
\hline-0.67 & 0 & 61 & $8.2 e-07$ & \\
\hline-0.43 & 0 & 75 & $3.2 e-08$ & \\
\hline-0.22 & 0 & 58 & $1.7 e-06$ & \\
\hline 0.31 & 0 & 75 & 3. $3 e-08$ & 1 \\
\hline-2.01 & 0 & 93 & $4.6 e-10$ & \\
\hline-1.56 & 0 & 121 & $7.1 e-13$ & \\
\hline-1.47 & 0 & 106 & 2. $3 e-11$ & \\
\hline-1.30 & 0 & 72 & $6.3 e-08$ & \\
\hline-0.54 & 0 & 106 & $2.7 e-11$ & 1 \\
\hline-0.24 & 0 & 55 & $2.9 e-06$ & \\
\hline-0.20 & 0 & 106 & $2.7 e-11$ & 1 \\
\hline-0.12 & 0 & 70 & $9.8 e-08$ & \\
\hline-0.12 & 0 & 100 & $1.1 \mathrm{e}-10$ & \\
\hline-0.031 & 0 & 128 & $1.6 e-13$ & \\
\hline 0.18 & 0 & 72 & $7 e-08$ & \\
\hline 1.52 & 0 & 54 & $4 e-06$ & \\
\hline 2.84 & 0 & 56 & $2.4 e-06$ & \\
\hline-0.49 & 0 & 61 & $7.5 e-07$ & 1 \\
\hline-1.79 & 0 & 67 & $2 e-07$ & 1 \\
\hline
\end{tabular}

Peptide

R. VETWLIONEOVK A

T R. VETWLLONEOVK. A

U R.VETWLLQNEQVK.A

U R. VETWLLONEOVK. A

U R.VETWLLQNEQVK. A

U R.VETWLLQNEQVK.A

U R.VETWLLQNEQVK. A

U R.VETWLLQNEQVK.A

U R.VETWLLQNEQVK.A

U R.VETWLLQNEQVK. A

U R.VETWLLQNEQVK.A

U R.VETWLLQNEQVK.A

U R.VETWLLONEOVK.A

U R.VETWLLQNEQVK.A

U R.VETWLLQNEQVK.A

U R.VETWLLQNEOVK. A

U R.VETWLLQNEQVK.A

U R.VETWLLQNEQVK.A

U K. AHIYGNTTDPR. I

U K. AHIYGNTTDPR. I

U K.AHIYGNTTDPR.I

U K.AHIYGNTTDPR. I

$U$ K.AHIYGNTTDPR.I

U K.AHIYGNTTDPR.I

U K. AHIYGNTTDPR. I

U K.AHIYGNTTDPR.I

U R.VIDEQVIGENGAEALMPEDR.V

U R. VVLLGEELGHTMEGPTVENNYKPGIYVIPEIK. E

U R. VVLLGEELGHTMEGPTVENNYKPGIYVIPEIK. E

U R. VVLLGEELGHTMEGPTVENNYKPGIYVIPEIK. E

U R.VVLLGEELGHTMEGPTVENNYKPGIYVIPEIK. E

U R.VVLLGEELGHTMEGPTVENNYKPGIYVIPEIK.E

U R. VVLLGEELGHTMEGPTVENNYKPGIYVIPEIK. E

U R. VVLLGEELGHTMEGPTVENNYKPGIYVIPEIK. E

U R.VVLLGEELGHTMEGPTVENNYKPGIYVIPEIK.E

U R. VVLLGEELGHTMEGPTVENNYKPGIYVIPEIK. E

U R.VVLLGEELGHTMEGPTVENNYKPGIYVIPEIK. E

U R. VVLLGEELGHTMEGPTVENNYKPGIYVIPEIK.E

U R. VVLLGEELGHTMEGPTVENNYKPGIYVIPEIK. E

U R. QEVYVGK.S

U K. SVFPALERPQAVVHLIVAQS. -
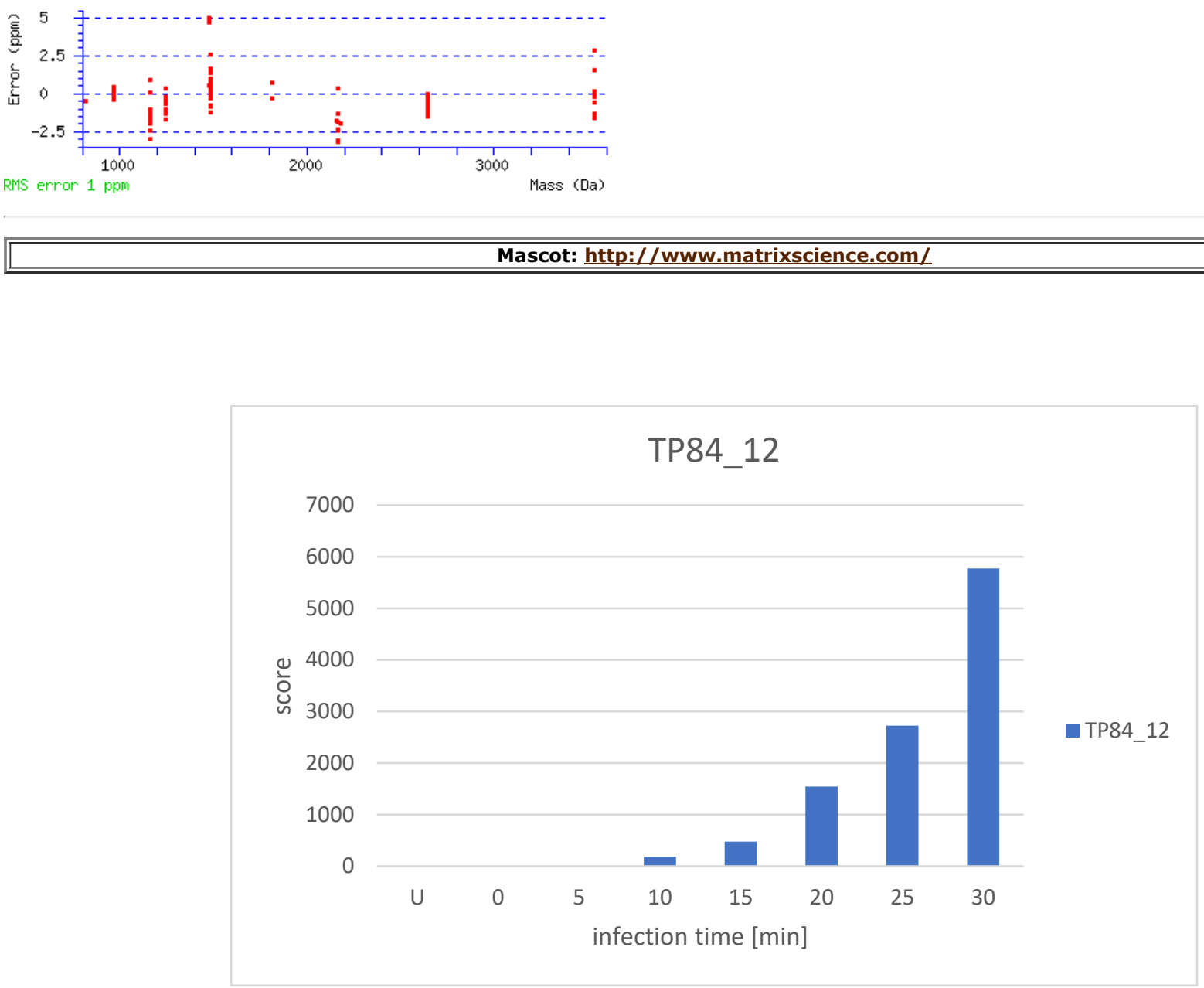


\title{
MASCOT Search Results
}

\section{Protein View: TP84_13}

\author{
Database: TP84 \\ Score: $\quad 428$ \\ Nominal mass $\left(M_{\mathbf{r}}\right): 7214$ \\ Calculated pI: $\quad 9.19$
}

Sequence similarity is available as an NCBI BLAST search of 68. against nr.

\section{Search parameters}

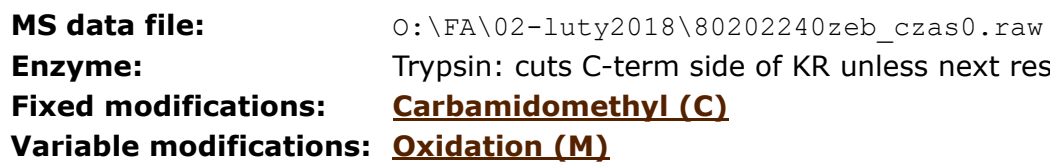

\section{Protein sequence coverage: $39 \%$}

Matched peptides shown in bold red.

1 MPKYIAKRHL VTRTGIKKPG DVIEYTKEQA QKLLAAGFIE EAEEEKKTTS

51 KGKQKADEAD KVDK

Unformatted sequence string: $\underline{\mathbf{6 4}}$ residues (for pasting into other applications).

Sort peptides by $\bullet$ Residue Number $\bigcirc$ Increasing Mass $\bigcirc$ Decreasing Mass

Show predicted peptides also

$\begin{array}{rcrrr}\text { Query } & \text { Start }- \text { End } & \text { Observed } & \text { Mr (expt) } & \text { Mr (calc) } \\ \varangle \underline{6701} & 18-27 & 575.3153 & 1148.6161 & 1148.6077 \\ \square \underline{19286} & 33-47 & 559.6305 & 1675.8698 & 1675.8668 \\ \square \underline{19287} & 33-47 & 559.6308 & 1675.8707 & 1675.8668 \\ \square \underline{19288} & 33-47 & 559.6318 & 1675.8735 & 1675.8668 \\ \square \underline{19289} & 33-47 & 559.6323 & 1675.8751 & 1675.8668\end{array}$

$\begin{array}{lrr}\text { Ppm } & \text { M } & \text { Score } \\ 7.31 & 0 & 56 \\ 1.76 & 1 & 104 \\ 2.32 & 1 & 105 \\ 4.00 & 1 & 101 \\ 4.97 & 1 & 114\end{array}$

$\begin{array}{lc}\text { Expect } & \text { Rank } \\ 2.7 e-06 & 1 \\ 4.2 e-11 & 1 \\ 3.1 e-11 & 1 \\ 7.2 e-11 & 1 \\ 4.2 e-12 & 1\end{array}$

U Peptide

Ü K.KPGDVIEYTK.E

U K. LLAAGFIEEAEEEKK. T

U K. LLAAGFIEEAEEEKK. T

$\checkmark 19289 \quad 33-47$

$559.6323 \quad 1675.8751 \quad 1675.8668$

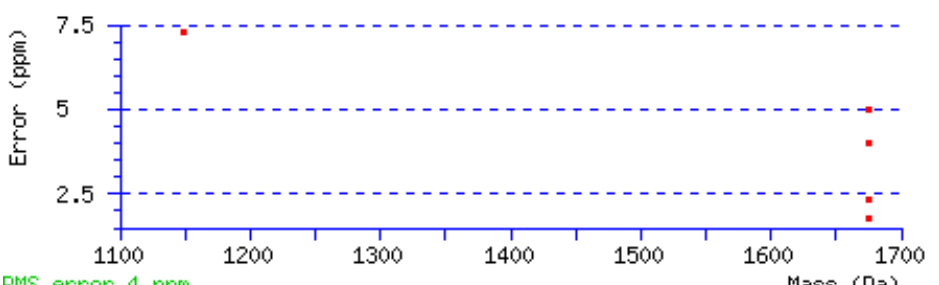

RMS error 4 ppm Mass (Da) 


\title{
MASCOT Search Results
}

\section{Protein View: TP84_13}

\author{
Database: TP84 \\ Score: $\quad 467$ \\ Nominal mass $\left(M_{\mathbf{r}}\right): 7214$ \\ Calculated pI: $\quad 9.19$
}

Sequence similarity is available as an NCBI BLAST search of 68. against nr.

\section{Search parameters}

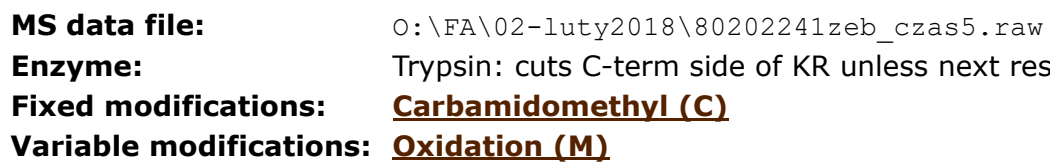

\section{Protein sequence coverage: $\mathbf{2 3} \%$}

Matched peptides shown in bold red.

1 MPKYIAKRHL VTRTGIKKPG DVIEYTKEQA QKLLAAGFIE EAEEEKKTTS

51 KGKQKADEAD KVDK

Unformatted sequence string: $\underline{\mathbf{6 4}}$ residues (for pasting into other applications).

Sort peptides by $\bullet$ Residue Number $\bigcirc$ Increasing Mass $\bigcirc$ Decreasing Mass

Show predicted peptides also

$\begin{array}{rr}\text { Query } & \text { Start }- \text { End } \\ \varangle 19287 & 33-47 \\ \varangle 19288 & 33-47 \\ \nabla 19289 & 33-47 \\ \nabla 19290 & 33-47 \\ \nabla 19291 & 33-47\end{array}$

$\begin{array}{rrr}\text { Observed } & \text { Mr(expt) } & \text { Mr (calc) } \\ 559.6299 & 1675.8679 & 1675.8668 \\ 559.6301 & 1675.8684 & 1675.8668 \\ 559.6304 & 1675.8694 & 1675.8668 \\ 559.6306 & 1675.8700 & 1675.8668 \\ 559.6311 & 1675.8714 & 1675.8668\end{array}$

$\begin{array}{llc}\text { Ppm } & \text { M } & \text { Score } \\ 0.65 & \dddot{1} & 106 \\ 0.98 & 1 & 102 \\ 1.53 & 1 & 110 \\ 1.89 & 1 & 110 \\ 2.73 & 1 & 91\end{array}$

$\begin{array}{rc}\text { Expect } & \text { Rank } \\ 2.4 e-11 & 1 \\ 5.7 e-11 & 1 \\ 1 e-11 & \frac{1}{\ldots} \\ 1.1 e-11 & 1 \\ 8.4 e-10 & \frac{1}{1}\end{array}$

U Peptide

Ü K. LLAAGFIEEAEEEKK. T U K. LLAAGFIEEAEEEKK. T $\begin{array}{ll}\text { U } & \text { K. LLAAGFIEEAEEEKK. T } \\ \text { U } & \text { K. LLAAGFIEEAEEEKK. T }\end{array}$

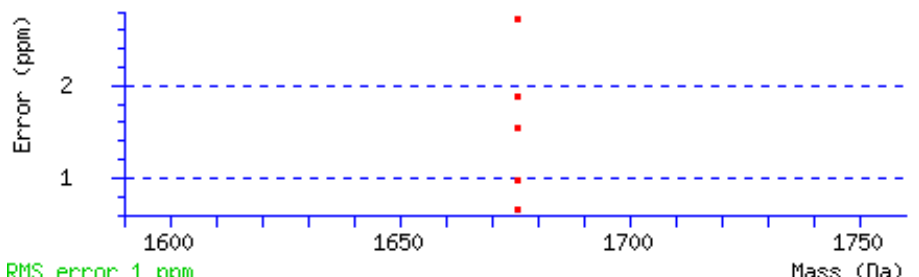




\title{
MASCOT Search Results
}

\section{Protein View: TP84_13}

\author{
Database: TP84 \\ Score: 182 \\ Nominal mass $\left(M_{\mathbf{r}}\right): 7214$ \\ Calculated pI: $\quad 9.19$
}

Sequence similarity is available as an NCBI BLAST search of 68. against nr.

\section{Search parameters}

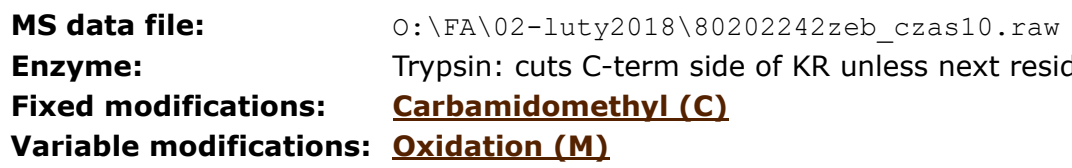

\section{Protein sequence coverage: $\mathbf{2 3} \%$}

Matched peptides shown in bold red.

1 MPKYIAKRHL VTRTGIKKPG DVIEYTKEQA QKLLAAGFIE EAEEEKKTTS

51 KGKQKADEAD KVDK

Unformatted sequence string: $\underline{\mathbf{6 4}}$ residues (for pasting into other applications).

Sort peptides by $\bullet$ Residue Number $\bigcirc$ Increasing Mass $\bigcirc$ Decreasing Mass

Show predicted peptides also

$\begin{array}{rcccc}\text { Query } & \text { Start }- \text { End } & \text { Observed } & \text { Mr (expt) } & \text { Mr (calc) } \\ \nabla^{\prime} 18390 & 33-47 & 559.6297 & 1675.8673 & 1675.8668 \\ \nabla^{\prime} 18391 & 33-47 & 559.6315 & 1675.8726 & 1675.8668\end{array}$

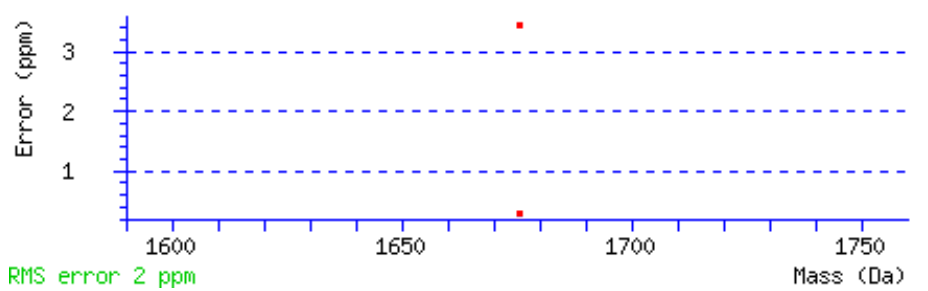




\title{
MASCOT Search Results
}

\section{Protein View: TP84_13}

\author{
Database: TP84 \\ Score: $\quad 837$ \\ Nominal mass $\left(M_{\mathbf{r}}\right): 7214$ \\ Calculated pI: $\quad 9.19$
}

Sequence similarity is available as an NCBI BLAST search of 68 . against nr.

\section{Search parameters}

\author{
MS data file: $\quad O: \backslash F A \backslash 02-1 u t y 2018 \backslash 80202244$ zeb_czas20.raw \\ Enzyme: Trypsin: cuts $\mathrm{C}$-term side of $\mathrm{KR}$ unless next residue is $\mathrm{P}$. \\ Fixed modifications: Carbamidomethyl (C) \\ Variable modifications: Oxidation (M)
}

\section{Protein sequence coverage: $39 \%$}

Matched peptides shown in bold red.

1 MPKYIAKRHL VTRTGIKKPG DVIEYTKEQA QKLLAAGFIE EAEEEKKTTS

51 KGKQKADEAD KVDK

Unformatted sequence string: $\mathbf{6 4}$ residues (for pasting into other applications).

Sort peptides by $\odot$ Residue Number $\bigcirc$ Increasing Mass $\bigcirc$ Decreasing Mass

Show predicted peptides also

\begin{tabular}{|c|c|}
\hline Query & Start - End \\
\hline$\nabla^{\prime 13175}$ & $18-27$ \\
\hline$\checkmark 13177$ & $18-27$ \\
\hline 13178 & $18-27$ \\
\hline$\sigma^{\prime} 13179$ & $18-27$ \\
\hline$\sigma^{30807}$ & $33-47$ \\
\hline$\checkmark 30808$ & $33-47$ \\
\hline$\checkmark 30809$ & $33-47$ \\
\hline$\sigma^{30810}$ & $33-47$ \\
\hline$\sigma^{\prime 30811}$ & $33-47$ \\
\hline$\sigma^{\prime 30812}$ & $33-47$ \\
\hline '30813 & $33-47$ \\
\hline
\end{tabular}

$\begin{array}{rrr}\text { Observed } & \text { Mr (expt) } & \text { Mr (calc) } \\ 575.3149 & 1148.6153 & 1148.6077 \\ 575.3152 & 1148.6157 & 1148.6077 \\ 575.3154 & 1148.6162 & 1148.6077 \\ 575.3157 & 1148.6168 & 1148.6077 \\ 559.6310 & 1675.8712 & 1675.8668 \\ 559.6311 & 1675.8715 & 1675.8668 \\ 559.6314 & 1675.8723 & 1675.8668 \\ 559.6314 & 1675.8724 & 1675.8668 \\ 559.6315 & 1675.8728 & 1675.8668 \\ 559.6317 & 1675.8731 & 1675.8668 \\ 559.6328 & 1675.8767 & 1675.8668\end{array}$

$\begin{array}{llr}\text { ppm } & \text { M } & \text { Score } \\ 6.60 & 0 & 81 \\ 6.98 & 0 & 74 \\ 7.37 & 0 & 62 \\ 7.89 & 0 & 70 \\ 2.62 & 1 & 104 \\ 2.82 & 1 & 82 \\ 3.30 & 1 & 90 \\ 3.36 & 1 & 100 \\ 3.55 & 1 & 107 \\ 3.77 & 1 & 87 \\ 5.90 & 1 & 109\end{array}$

$\begin{array}{rc}\text { Expect } & \text { Rank } \\ 8.1 e-09 & 1 \\ 4.4 e-08 & 1 \\ 5.9 e-07 & \frac{1}{1} \\ 1 e-07 & 1 \\ 3.9 e-11 & -1 \\ 6.4 e-09 & 1 \\ 9.1 e-10 & \frac{1}{.} \\ 9.1 e-11 & 1 \\ 2.1 e-11 & 1 \\ 1.9 e-09 & 1 \\ 1.1 e-11 & 1\end{array}$

U Peptide

Ü K.KPGDVIEYTK.E

U K. KPGDVIEYTK.E

U K.KPGDVIEYTK.E

U K.KPGDVIEYTK.E

U K. LLAAGFIEEAEEEKK.T

U K. LLAAGFIEEAEEEKK. T

U K. LLAAGFIEEAEEEKK. T

U K. LLAAGFIEEAEEEKK. T

U K. LLAAGFIEEAEEEKK. T

U K. LLAAGFIEEAEEEKK.T

$\begin{array}{lll}559.6328 & 1675.8767 & 1675.8668\end{array}$

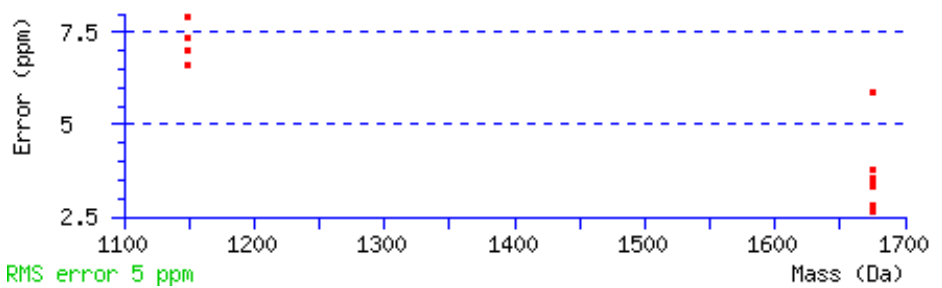




\title{
MASCOT Search Results
}

\section{Protein View: TP84_13}

\author{
Database: TP84 \\ Score: 1553 \\ Nominal mass $\left(M_{\mathbf{r}}\right): 7214$ \\ Calculated pI: $\quad 9.19$
}

Sequence similarity is available as an NCBI BLAST search of 68. against nr.

\section{Search parameters}

\author{
MS data file: $\quad 0: \backslash F A \backslash 02-1 u t y 2018 \backslash 80202245 z e b \_c z a s 25 . r a w$ \\ Enzyme: Trypsin: cuts C-term side of KR unless next residue is $P$. \\ Fixed modifications: Carbamidomethyl (C) \\ Variable modifications: Oxidation (M)
}

\section{Protein sequence coverage: $53 \%$}

Matched peptides shown in bold red.

1 MPKYIAKRHL VTRTGIKKPG DVIEYTKEQA QKLLAAGFIE EAEEEKKTTS

51 KGKQKADEAD KVDK

Unformatted sequence string: 64 residues (for pasting into other applications).

Sort peptides by $\odot$ Residue Number $\bigcirc$ Increasing Mass $\bigcirc$ Decreasing Mass

Show predicted peptides also

\begin{tabular}{|c|c|}
\hline Query & Start - End \\
\hline$\checkmark 28273$ & $18-27$ \\
\hline$\overline{8276}$ & $18-27$ \\
\hline$\sigma^{\prime 18895}$ & $33-46$ \\
\hline$\overline{18896}$ & $33-46$ \\
\hline 18897 & $33-46$ \\
\hline 721836 & $33-47$ \\
\hline$\checkmark 21837$ & $33-47$ \\
\hline 21838 & $33-47$ \\
\hline$\checkmark 21839$ & $33-47$ \\
\hline$\checkmark 21840$ & $33-47$ \\
\hline$\sigma^{21841}$ & $33-47$ \\
\hline$\checkmark 21842$ & $33-47$ \\
\hline$\checkmark 21843$ & $33-47$ \\
\hline$\sigma^{21844}$ & $33-47$ \\
\hline$\nabla^{21845}$ & $33-47$ \\
\hline$\checkmark 21846$ & $33-47$ \\
\hline$\overline{4688}$ & $56-64$ \\
\hline
\end{tabular}

$\begin{array}{rrr}\text { Observed } & \text { Mr(expt) } & \text { Mr (calc) } \\ 575.3114 & 1148.6082 & 1148.6077 \\ 575.3122 & 1148.6099 & 1148.6077 \\ 774.8919 & 1547.7693 & 1547.7719 \\ 774.8931 & 1547.7716 & 1547.7719 \\ 774.8935 & 1547.7724 & 1547.7719 \\ 559.6291 & 1675.8656 & 1675.8668 \\ 559.6292 & 1675.8659 & 1675.8668 \\ 559.6293 & 1675.8660 & 1675.8668 \\ 559.6296 & 1675.8669 & 1675.8668 \\ 559.6296 & 1675.8670 & 1675.8668 \\ 559.6296 & 1675.8671 & 1675.8668 \\ 559.6297 & 1675.8673 & 1675.8668 \\ 559.6297 & 1675.8674 & 1675.8668 \\ 559.6298 & 1675.8677 & 1675.8668 \\ 559.6300 & 1675.8683 & 1675.8668 \\ 559.6301 & 1675.8684 & 1675.8668 \\ 495.7409 & 989.4673 & 989.4665\end{array}$

\begin{tabular}{rrr}
\multicolumn{1}{c}{ ppm } & M & Score \\
0.45 & 0 & 87 \\
1.93 & 0 & 97 \\
-1.67 & 0 & 98 \\
-0.13 & 0 & 116 \\
0.36 & 0 & 102 \\
-0.74 & 1 & 114 \\
-0.55 & 1 & 104 \\
-0.51 & 1 & 112 \\
0.063 & 1 & 99 \\
0.099 & 1 & 106 \\
0.19 & 1 & 103 \\
0.31 & 1 & 106 \\
0.37 & 1 & 115 \\
0.53 & 1 & 118 \\
0.87 & 1 & 110 \\
0.94 & 1 & 101 \\
0.82 & 1 & 74
\end{tabular}

\begin{tabular}{|c|c|}
\hline Expect & Rank \\
\hline $2 e-09$ & 1 \\
\hline $1 e-10$ & 1 \\
\hline $6 e-10$ & 1 \\
\hline $.6 e-12$ & 1 \\
\hline $.5 e-11$ & 1 \\
\hline $.8 e-12$ & 1 \\
\hline $4.1 e-11$ & 1 \\
\hline $6.3 e-12$ & 1 \\
\hline 1. $4 \mathrm{e}-10$ & 1 \\
\hline $2.3 e-11$ & 1 \\
\hline $4.8 e-11$ & 1 \\
\hline $2.5 e-11$ & 1 \\
\hline 3. $5 e-12$ & 1 \\
\hline $1.7 e-12$ & 1 \\
\hline $1 e-11$ & 1 \\
\hline $7.5 e-11$ & 1 \\
\hline 3. $6 e-08$ & 1 \\
\hline
\end{tabular}

U Peptide

Ü K.KPGDVIEYTK.E

U K.KPGDVIEYTK.E

U K. LLAAGFIEEAEEEK. K

U K. LLAAGFIEEAEEEK. K

U K. LLAAGFIEEAEEEK.K

U K. LLAAGFIEEAEEEKK. T

U K. LLAAGFIEEAEEEKK.T

U K. LLAAGFIEEAEEEKK. T

U K. LLAAGFIEEAEEEKK. T

U K. LLAAGFIEEAEEEKK. T

U K. LLAAGFIEEAEEEKK. T

U K. LLAAGF IEEAEEEKK. T

U K. LLAAGFIEEAEEEKK. T

U K. LLAAGF IEEAEEEKK. T

U K. LLAAGFIEEAEEEKK. T

U K. LLAAGFIEEAEEEKK.T

U K.ADEADKVDK.-

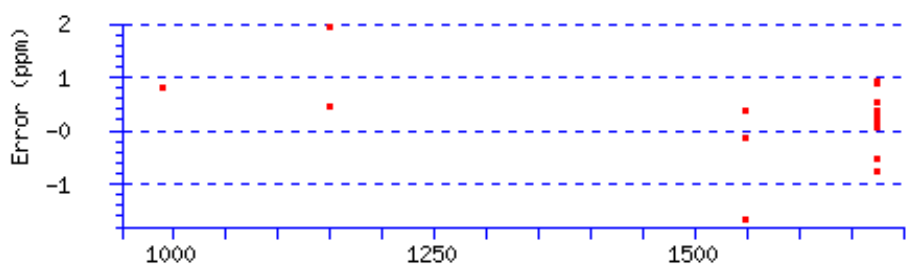

RMS error 0 ppm

Mass (Da) 
MASCOT Search Results

\title{
Protein View: TP84_13
}

\author{
Database: TP84 \\ Score: 2311 \\ Nominal mass $\left(M_{\mathbf{r}}\right): 7214$ \\ Calculated pI: $\quad 9.19$
}

Sequence similarity is available as an NCBI BLAST search of 68. against nr.

\section{Search parameters}

\author{
MS data file: \\ Enzyme: \\ $0: \backslash F A \backslash 02-1 u t y 2018 \backslash 80202246 z e b$ czas30.raw \\ Fixed modifications: \\ Trypsin: cuts C-term side of KR unless next residue is $P$. \\ Variable modifications: Oxidation (M)
}

\section{Protein sequence coverage: $39 \%$}

Matched peptides shown in bold red.

1 MPKYIAKRHL VTRTGIKKPG DVIEYTKEQA QKLLAAGFIE EAEEEKKTTS

51 KGKQKADEAD KVDK

Unformatted sequence string: 64 residues (for pasting into other applications).

Sort peptides by $\odot$ Residue Number $\bigcirc$ Increasing Mass $\bigcirc$ Decreasing Mass

Show predicted peptides also

\begin{tabular}{|c|c|}
\hline Query & Start - End \\
\hline$\nabla^{\prime \prime 11411}$ & $18-27$ \\
\hline$\overline{11415}$ & $18-27$ \\
\hline$\overline{11416}$ & $18-27$ \\
\hline$\overline{11417}$ & $18-27$ \\
\hline$\overline{11418}$ & $18-27$ \\
\hline$\overline{11419}$ & $18-27$ \\
\hline$\sigma^{\prime 1420}$ & $18-27$ \\
\hline$\triangle 11421$ & $18-27$ \\
\hline$\sigma^{\prime 1422}$ & $18-27$ \\
\hline$\checkmark 26024$ & $33-46$ \\
\hline$\checkmark 26025$ & $33-46$ \\
\hline$\checkmark 26026$ & $33-46$ \\
\hline$\checkmark 26027$ & $33-46$ \\
\hline$\checkmark ́ 26028$ & $33-46$ \\
\hline$\checkmark 26029$ & $33-46$ \\
\hline$\checkmark 26030$ & $33-46$ \\
\hline$\nabla^{\prime 26031}$ & $33-46$ \\
\hline$\checkmark \underline{29702}$ & $33-47$ \\
\hline$\checkmark \underline{29703}$ & $33-47$ \\
\hline$\checkmark \overline{29704}$ & $33-47$ \\
\hline$\checkmark 29705$ & $33-47$ \\
\hline$\nabla^{\prime 29706}$ & $33-47$ \\
\hline$\square^{299708}$ & $33-47$ \\
\hline$\checkmark 29709$ & $33-47$ \\
\hline$\checkmark 29710$ & $33-47$ \\
\hline$\underbrace{\prime 29711}$ & $33-47$ \\
\hline$\mho^{\prime 29712}$ & $33-47$ \\
\hline$\checkmark 29713$ & $33-47$ \\
\hline
\end{tabular}

$\begin{array}{rrr}\text { Observed } & \text { Mr(expt) } & \text { Mr (calc) } \\ 383.8762 & 1148.6069 & 1148.6077 \\ 575.3114 & 1148.6083 & 1148.6077 \\ 575.3115 & 1148.6084 & 1148.6077 \\ 575.3116 & 1148.6087 & 1148.6077 \\ 575.3118 & 1148.6090 & 1148.6077 \\ 575.3118 & 1148.6091 & 1148.6077 \\ 575.3119 & 1148.6091 & 1148.6077 \\ 575.3119 & 1148.6093 & 1148.6077 \\ 575.3121 & 1148.6097 & 1148.6077 \\ 774.8878 & 1547.7610 & 1547.7719 \\ 774.8909 & 1547.7673 & 1547.7719 \\ 774.8924 & 1547.7702 & 1547.7719 \\ 774.8926 & 1547.7706 & 1547.7719 \\ 774.8931 & 1547.7716 & 1547.7719 \\ 774.8933 & 1547.7720 & 1547.7719 \\ 774.8939 & 1547.7732 & 1547.7719 \\ 774.8939 & 1547.7733 & 1547.7719 \\ 559.6292 & 1675.8657 & 1675.8668 \\ 559.6294 & 1675.8663 & 1675.8668 \\ 559.6296 & 1675.8669 & 1675.8668 \\ 559.6296 & 1675.8669 & 1675.8668 \\ 559.6297 & 1675.8674 & 1675.8668 \\ 559.6298 & 1675.8677 & 1675.8668 \\ 559.6300 & 1675.8681 & 1675.8668 \\ 559.6301 & 1675.8684 & 1675.8668 \\ 559.6307 & 1675.8702 & 1675.8668 \\ 559.6307 & 1675.8703 & 1675.8668 \\ 559.6309 & 1675.8708 & 1675.8668\end{array}$

\begin{tabular}{rrr}
\multicolumn{1}{c}{ ppm } & M & Score \\
-0.73 & 0 & 53 \\
0.47 & 0 & 97 \\
0.63 & 0 & 82 \\
0.84 & 0 & 82 \\
1.08 & 0 & 92 \\
1.22 & 0 & 86 \\
1.24 & 0 & 92 \\
1.34 & 0 & 86 \\
1.71 & 0 & 97 \\
-6.98 & 0 & 55 \\
-2.94 & 0 & 87 \\
-1.07 & 0 & 100 \\
-0.82 & 0 & 99 \\
-0.18 & 0 & 119 \\
0.11 & 0 & 108 \\
0.85 & 0 & 116 \\
0.94 & 0 & 118 \\
-0.65 & 1 & 94 \\
-0.29 & 1 & 106 \\
0.027 & 1 & 101 \\
0.063 & 1 & 91 \\
0.37 & 1 & 99 \\
0.51 & 1 & 103 \\
0.80 & 1 & 106 \\
0.96 & 1 & 94 \\
2.05 & 1 & 103 \\
2.10 & 1 & 101 \\
2.37 & 1 & 96
\end{tabular}

Expect Rank U Peptide

4. $5 e-061$

U K.KPGDVIEYTK.E

2. $2 e-10$

U K.KPGDVIEYTK.E

$6.4 e-09 \quad 1$

6. $6 e-091$

U K.KPGDVIEYTK.E

U K.KPGDVIEYTK.E

7.1e-10 1 U K.KPGDVIEYTK.E

2.7e-09 1 U K.KPGDVIEYTK.E

7e-10 1 U K.KPGDVIEYTK.E

2.6e-09 1 U K.KPGDVIEYTK.E

$2.1 \mathrm{e}-10$ 1 $\frac{1}{1}$ U K.KPGDVIEYTK.E

3.5e-06 1 U K.LLAAGFIEEAEEEK.K

2e-09 1 U K. LLAAGFIEEAEEEK.K

9.5e-11 1 U K.LLAAGFIEEAEEEK.K

$1.4 \mathrm{e}-10 \quad 1$ U K. LLAAGFIEEAEEEK.K

$1.3 e-12 \quad 1$ U K.LLAAGFIEEAEEEK.K

$1.5 e-11 \quad 1 \quad$ U K. LLAAGFIEEAEEEK.K

2.3e-12 1 U K.LLAAGFIEEAEEEK.K

$1.5 e-12 \quad 1$ U K.LLAAGFIEEAEEEK.K

$4.4 \mathrm{e}-10 \quad \frac{1}{1}$ U K. LLAAGFIEEAEEEKK.T

2.5e-11 1 U K. LLAAGFIEEAEEEKK.T

$7.6 e-11 \quad 1$ U K.LLAAGFIEEAEEEKK.T

7.3e-10 1 U K. LLAAGFIEEAEEEKK.T

$1.4 \mathrm{e}-10 \quad 1$ U K. LLAAGFIEEAEEEKK.T

$4.9 e-11$ U U K.LLAAGFIEEAEEEKK.T

2. 4 e-11 1 U K.LLAAGFIEEAEEEKK.T

$3.8 e-10 \quad \frac{1}{1}$ U K. LLAAGFIEEAEEEKK.T

4.9e-11 1 U K. LLAAGFIEEAEEEKK.T

7. $3 e-11 \quad 1 \quad$ U K. LLAAGFIEEAEEEKK.T

$2.3 e-10 \quad 1 \quad$ U K. LLAAGFIEEAEEEKK.T

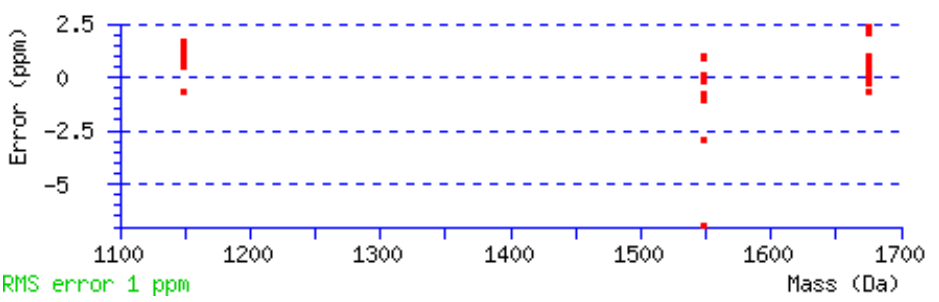




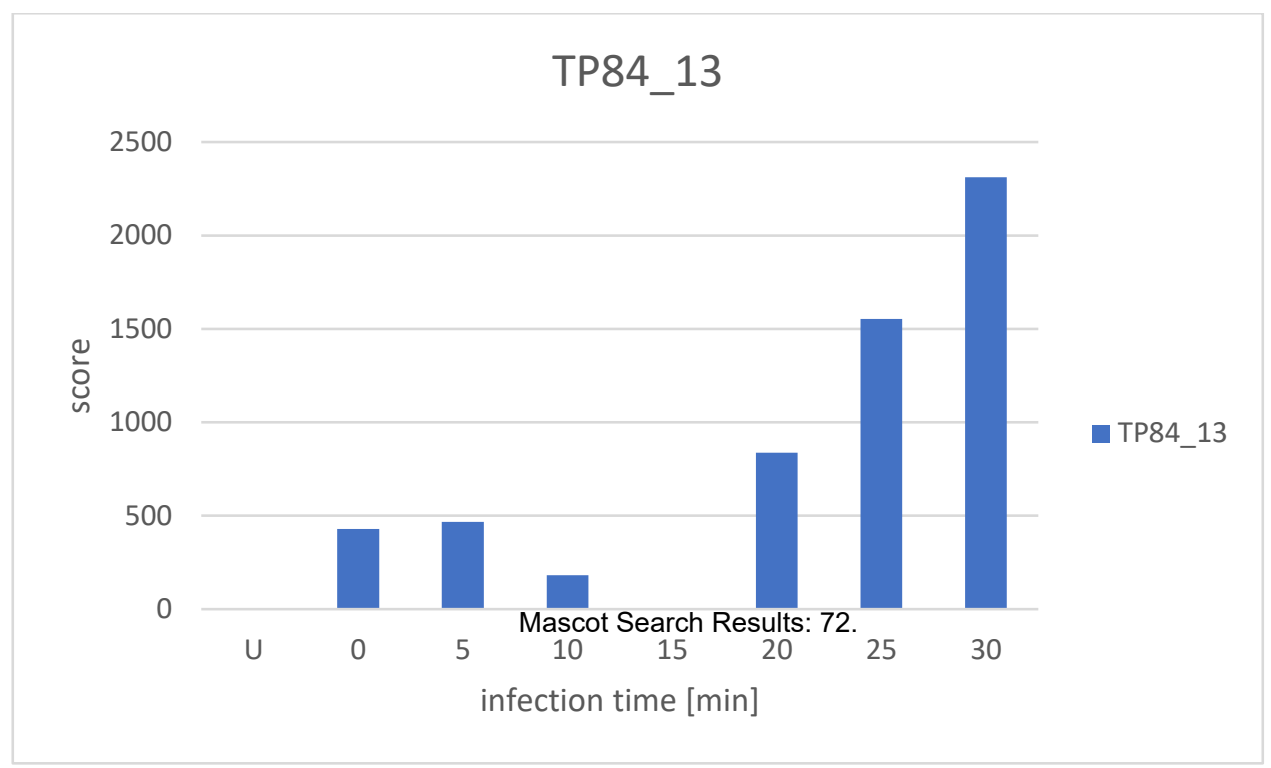

12.02.2018

\section{MASCOT Search Results}

\section{Protein View: TP84_17}

$\begin{array}{ll}\text { Database: } & \text { TP84 } \\ \text { Score: } & 246 \\ \text { Nominal mass }\left(M_{\mathbf{r}}\right): & 14639 \\ \text { Calculated pI: } & 5.35\end{array}$

Sequence similarity is available as an NCBI BLAST search of 72 . against $\mathrm{nr}$.

\section{Search parameters}

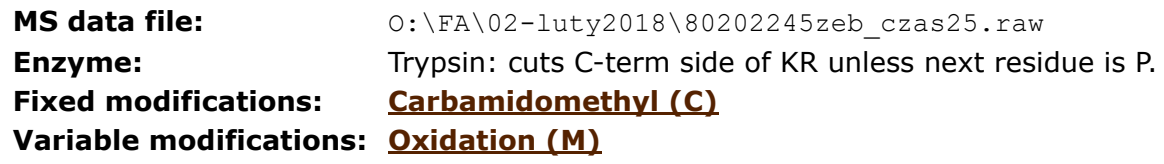

Protein sequence coverage: $\mathbf{2 0 \%}$

Matched peptides shown in bold red.

1 MIQKWIKDQL EAAIPNLEWT YDYKTGKDHT GVVYHETPGQ ISRDDFEIIT

51 PSYSVYIETS DMKNAEKWAW IVYDTMNKRR QEVATIDDRS FQVIFIEVTT

101 PPILVGIVDK KMTYSINLQA TIRKI

Unformatted sequence string: 125 residues (for pasting into other applications).

Sort peptides by $\odot$ Residue Number $\bigcirc$ Increasing Mass $\bigcirc$ Decreasing Mass

Show predicted peptides also

\begin{tabular}{|c|c|c|c|c|c|c|c|c|c|c|c|}
\hline Query & Start - End & Observed & $M r(\exp t)$ & $\operatorname{Mr}(\mathrm{calc})$ & ppm & $\mathbf{M}$ & Score & Expect & Rank & $\mathrm{U}$ & Peptide \\
\hline$\checkmark \underline{29123}$ & $8-24$ & 1034.9977 & 2067.9808 & 2067.9789 & 0.94 & $\dddot{0}$ & 108 & $1.5 e-11$ & 1 & $\dddot{\mathrm{U}}$ & K.DQLEAAI PNLEWTYDYK. T \\
\hline$\checkmark \underline{29124}$ & $8-24$ & 1034.9982 & 2067.9818 & 2067.9789 & 1.43 & 0 & 88 & $1.7 e-09$ & 1 & $\mathrm{U}$ & K.DQLEAAIPNLEWTYDYK. T \\
\hline$\checkmark 5934$ & $81-89$ & 523.7593 & 1045.5041 & 1045.5040 & 0.12 & 0 & 76 & $2.7 e-08$ & 1 & $\mathrm{U}$ & R.QEVATIDDR.S \\
\hline
\end{tabular}

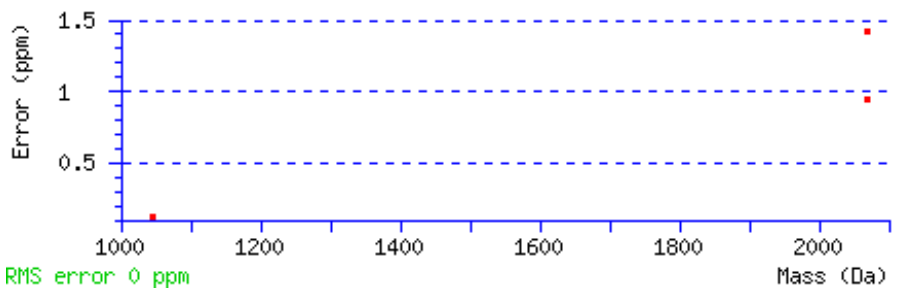




\title{
MASCOT Search Results
}

\section{Protein View: TP84_17}

\author{
Database: $\quad$ TP84 \\ Score: $\quad 451$ \\ Nominal mass $\left(\mathbf{M}_{\mathbf{r}}\right)$ : 14639 \\ Calculated pI: $\quad 5.35$
}

Sequence similarity is available as an NCBI BLAST search of 72. against nr.

\section{Search parameters}

\author{
MS data file: \\ Enzyme: \\ $0: \backslash F A \backslash 02-1$ uty2018\80202246zeb_czas30.raw \\ Fixed modifications: \\ Trypsin: cuts C-term side of KR unless next residue is $\mathrm{P}$. \\ Carbamidomethyl (C) \\ Variable modifications: Oxidation (M)
}

\section{Protein sequence coverage: $\mathbf{2 0 \%}$}

Matched peptides shown in bold red.

1 MIQKWIKDQL EAAIPNLEWT YDYKTGKDHT GVVYHETPGQ ISRDDFEIIT

51 PSYSVYIETS DMKNAEKWAW IVYDTMNKRR QEVATIDDRS FQVIFIEVTT

101 PPILVGIVDK KMTYSINLQA TIRKI

Unformatted sequence string: 125 residues (for pasting into other applications).

Sort peptides by $\odot$ Residue Number $\bigcirc$ Increasing Mass $\bigcirc$ Decreasing Mass

Show predicted peptides also

\begin{tabular}{|c|c|}
\hline Query & Start - End \\
\hline ‘33001 & $28-43$ \\
\hline$\sigma^{\prime} 33002$ & $28-43$ \\
\hline$\overline{33003}$ & $28-43$ \\
\hline 33004 & $28-43$ \\
\hline$\checkmark \underline{33005}$ & $28-43$ \\
\hline$\smile^{\prime 7} 7925$ & $81-89$ \\
\hline$\checkmark 7926$ & $81-89$ \\
\hline
\end{tabular}

$\begin{array}{rrr}\text { Observed } & \text { Mr (expt) } & M r \text { (calc) } \\ 898.4375 & 1794.8605 & 1794.8649 \\ 898.4394 & 1794.8642 & 1794.8649 \\ 599.2956 & 1794.8650 & 1794.8649 \\ 599.2960 & 1794.8663 & 1794.8649 \\ 599.2961 & 1794.8664 & 1794.8649 \\ 523.7592 & 1045.5038 & 1045.5040 \\ 523.7595 & 1045.5045 & 1045.5040\end{array}$

\begin{tabular}{rrr}
\multicolumn{1}{c}{ ppm } & M & Score \\
-2.45 & 0 & 74 \\
-0.40 & 0 & 120 \\
0.024 & 0 & 80 \\
0.76 & 0 & 53 \\
0.81 & 0 & 71 \\
-0.14 & 0 & 66 \\
0.47 & 0 & 64
\end{tabular}

$\begin{array}{rc}\text { Expect } & \text { Rank } \\ 3.6 e-08 & 1 \\ 1.1 e-12 & 1 \\ 9.1 e-09 & 1 \\ 4.5 e-06 & \frac{1}{1} \\ 7.7 e-08 & 1 \\ 2.5 e-07 & \frac{1}{1} \\ 3.8 e-07 & 1\end{array}$

U Peptide

Ü K.DHTGVVYHETPGQISR.D

U K.DHTGVVYHETPGQISR.D

U K.DHTGVVYHETPGQISR.D

U K.DHTGVVYHETPGQISR.D

U K.DHTGVVYHETPGQISR.D

$523.7595 \quad 1045.5045 \quad 1045.5040$

0.470

U R.QEVATIDDR.S

U R.QEVATIDDR.S
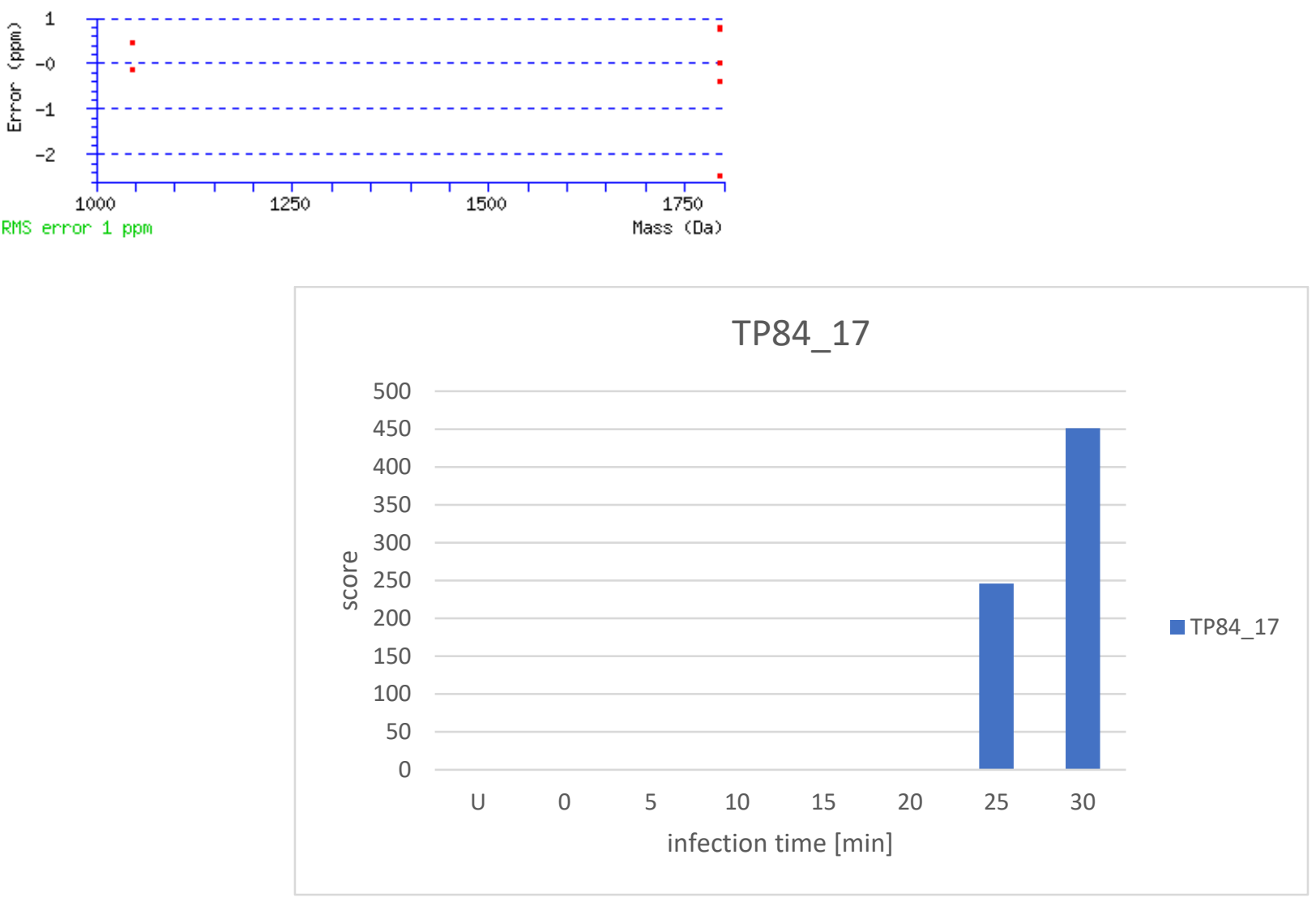


\section{MASCOT Search Results}

\section{Protein View: TP84_18}

$\begin{array}{ll}\text { Database: } & \text { TP84 } \\ \text { Score: } & 743 \\ \text { Nominal mass (Mr): } & 20371 \\ \text { Calculated pI: } & 4.38\end{array}$

Sequence similarity is available as an NCBI BLAST search of 73. against nr.

\section{Search parameters}

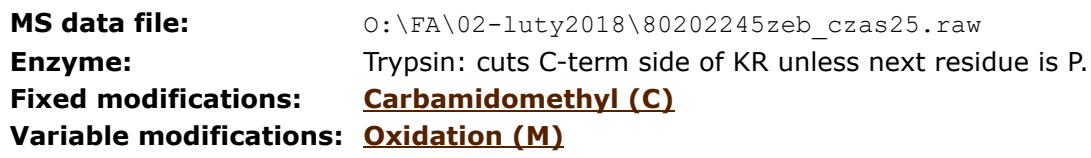

\section{Protein sequence coverage: $49 \%$}

Matched peptides shown in bold red.

1 MPGVNIPFGL ATITVGDPQD PNKIVFDGVN YFQAEGGELN IEPELEPIEL

51 QDFGATPYDE RINGYTCELT IVVGQNDLKL MRKLFAYHSE IVDEGTGEVI 101 GLTDEKIGAS MRDKAVPVTI HPREMGSDTS LDIHIYKMSG VGAFNRTYEN 151 AQGSYEVTLR AYPRDGADPT KPGNFYYIGD TDPNA

Unformatted sequence string: 185 residues (for pasting into other applications).

Sort peptides by $\odot$ Residue Number $\bigcirc$ Increasing Mass $\bigcirc$ Decreasing Mass

Show predicted peptides also

\begin{tabular}{|c|c|c|c|c|}
\hline Query & Start - End & Observed & $M r($ expt) & $\mathrm{Mr}$ (calc) \\
\hline$\sigma^{\prime 32901}$ & $1-23$ & 794.4100 & 2380.2083 & 2380.2097 \\
\hline 32902 & $1-23$ & 794.4109 & 2380.2108 & 2380.2097 \\
\hline$\checkmark 32903$ & $1-23$ & 794.4118 & 2380.2137 & 2380.2097 \\
\hline$\checkmark 34506$ & $83-106$ & 884.1130 & 2649.3171 & 2649.3174 \\
\hline$\checkmark 33808$ & $84-106$ & 841.4135 & 2521.2186 & 2521.2224 \\
\hline$\checkmark \underline{4687}$ & $115-123$ & 495.3005 & 988.5865 & 988.5818 \\
\hline$\square^{\prime 2} \underline{0692}$ & $147-160$ & 815.8891 & 1629.7636 & 1629.7634 \\
\hline$\checkmark 31193$ & $165-185$ & 1114.4889 & 2226.9632 & 2226.9706 \\
\hline$\checkmark 31195$ & $165-185$ & 1114.4898 & 2226.9650 & 2226.9706 \\
\hline
\end{tabular}

\begin{tabular}{rrrrr}
\multicolumn{1}{c}{ Ppm } & M Score & Expect & Rank \\
-0.59 & 0 & 71 & $7.9 \mathrm{e}-08$ & 1 \\
0.45 & 0 & 77 & $2.1 \mathrm{e}-08$ & 1 \\
1.66 & 0 & 58 & $1.5 \mathrm{e}-06$ & 1 \\
-0.11 & 1 & 137 & $2.2 \mathrm{e}-14$ & 1 \\
-1.50 & 0 & 117 & $2.1 \mathrm{e}-12$ & 1 \\
4.76 & 0 & 55 & $3.3 \mathrm{e}-06$ & 1 \\
0.10 & 0 & 104 & $4.2 \mathrm{e}-11$ & 1 \\
-3.29 & 0 & 117 & $1.9 \mathrm{e}-12$ & 1 \\
-2.48 & 0 & 111 & $7.3 \mathrm{e}-12$ & 1
\end{tabular}

U Peptide

U - .MPGVNIPFGLATITVGDPQDPNK. I

U -.MPGVNIPFGLATITVGDPQDPNK. I

U - .MPGVNIPFGLATITVGDPQDPNK. I

U R. KLFAYHSEIVDEGTGEVIGLTDEK. I

U K. LFAYHSEIVDEGTGEVIGLTDEK. I

U K.AVPVTIHPR.E

U R. TYENAQGSYEVTLR. A

U R.DGADPTKPGNFYYIGDTDPNA.--

U R.DGADPTKPGNFYYIGDTDPNA. -

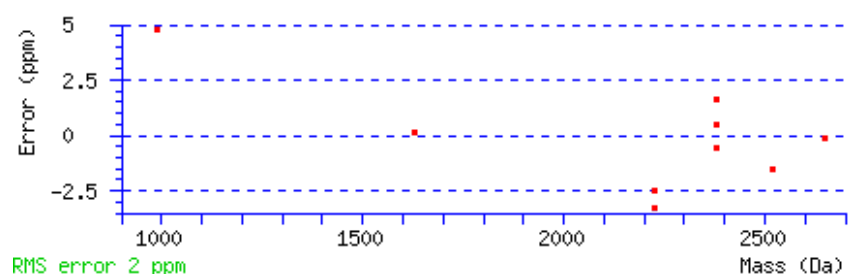




\title{
MASCOT Search Results
}

\section{Protein View: TP84_18}

\author{
Database: TP84 \\ Score: $\quad 648$ \\ Nominal mass $\left(\mathbf{M}_{\mathbf{r}}\right): 20371$ \\ Calculated pI: $\quad 4.38$
}

Sequence similarity is available as an NCBI BLAST search of 73. against nr.

\section{Search parameters}

MS data file: $\quad 0: \backslash F A \backslash 02-1 u t y 2018 \backslash 80202246 z e b \_c z a s 30 . r a w$

Enzyme: $\quad$ Trypsin: cuts C-term side of KR unless next residue is $P$.

Fixed modifications: Carbamidomethyl (C)

Variable modifications: Oxidation (M)

\section{Protein sequence coverage: $34 \%$}

Matched peptides shown in bold red.

1 MPGVNIPFGL ATITVGDPQD PNKIVFDGVN YFQAEGGELN IEPELEPIEL

51 QDFGATPYDE RINGYTCELT IVVGQNDLKL MRKLFAYHSE IVDEGTGEVI

101 GLTDEKIGAS MRDKAVPVTI HPREMGSDTS LDIHIYKMSG VGAFNRTYEN 151 AQGSYEVTLR AYPRDGADPT KPGNFYYIGD TDPNA

Unformatted sequence string: 185 residues (for pasting into other applications).

Sort peptides by $\bullet$ Residue Number $\bigcirc$ Increasing Mass $\bigcirc$ Decreasing Mass

Show predicted peptides also

\begin{tabular}{|c|c|c|c|c|}
\hline Query & Start - End & Observed & $M r$ (expt) & $\operatorname{Mr}(\mathrm{calc})$ \\
\hline$\sigma^{\prime 4} 4378$ & $83-106$ & 884.1135 & 2649.3186 & 2649.3174 \\
\hline 6013 & $115-123$ & 330.5344 & 988.5815 & 988.5818 \\
\hline$\triangle 6014$ & $115-123$ & 495.2982 & 988.5819 & 988.5818 \\
\hline$\triangle 6015$ & $115-123$ & 495.2988 & 988.5831 & 988.5818 \\
\hline$\triangle 4306$ & $138-146$ & 469.7285 & 937.4425 & 937.4440 \\
\hline$\triangle 4307$ & $138-146$ & 469.7286 & 937.4427 & 937.4440 \\
\hline$\square 4309$ & $138-146$ & 469.7288 & 937.4430 & 937.4440 \\
\hline$\triangle 4310$ & $138-146$ & 469.7292 & 937.4438 & 937.4440 \\
\hline 4311 & $138-146$ & 469.7292 & 937.4438 & 937.4440 \\
\hline$\sigma^{\prime} 40590$ & $165-185$ & 1114.4877 & 2226.9608 & 2226.9706 \\
\hline$\varangle 40591$ & $165-185$ & 1114.4933 & 2226.9720 & 2226.970 \\
\hline
\end{tabular}

\begin{tabular}{rrr}
\multicolumn{1}{c}{ ppm } & M & Score \\
0.46 & 1 & 121 \\
-0.34 & 0 & 52 \\
0.067 & 0 & 67 \\
1.30 & 0 & 59 \\
-1.62 & 0 & 60 \\
-1.36 & 0 & 58 \\
-1.00 & 0 & 57 \\
-0.21 & 0 & 53 \\
-0.19 & 0 & 53 \\
-4.36 & 0 & 101 \\
0.67 & 0 & 96
\end{tabular}

$\begin{array}{rc}\text { Expect } & \text { Rank } \\ 8.1 e-13 & 1 \\ 6.2 e-06 & \frac{1}{1} \\ 1.9 e-07 & \frac{1}{1} \\ 1.2 e-06 & \frac{1}{\cdots} \\ 9.6 e-07 & 1 \\ 1.7 e-06 & \frac{1}{\cdots} \\ 1.9 e-06 & 1 \\ 5.5 e-06 & \frac{1}{.} \\ 5.5 e-06 & \frac{1}{7 .} \\ 7.1 e-11 & 1 \\ 2.3 e-10 & \frac{1}{1}\end{array}$

U Peptide

U R. KLFAYHSEIVDEGTGEVIGLTDEK. I

U K.AVPVTIHPR.E

U K.AVPVTIHPR.E

U K.AVPVTIHPR.E

U K.MSGVGAFNR. T

U K.MSGVGAFNR. T

U K.MSGVGAFNR. T

U K.MSGVGAFNR. T

U K.MSGVGAFNR. T

U R.DGADPTKPGNFYYIGDTDPNA. -

$1114.4933 \quad 2226.9720 \quad 2226.9706$

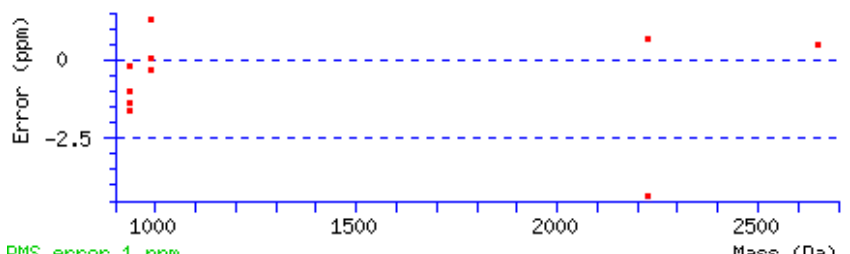

\section{TP84_18}

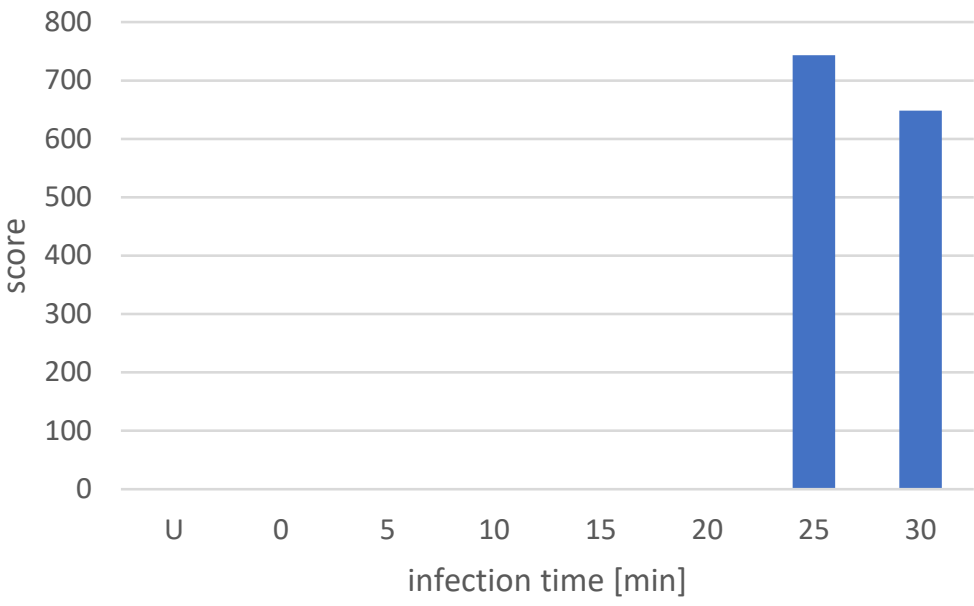




\title{
MASCOT Search Results
}

\section{Protein View: TP84_19}

\author{
Database: TP84 \\ Score: 1463 \\ Nominal mass $\left(\mathbf{M}_{\mathbf{r}}\right)$ : 19335 \\ Calculated pI: $\quad 4.90$
}

Sequence similarity is available as an NCBI BLAST search of 74. against $\mathrm{nr}$.

\section{Search parameters}

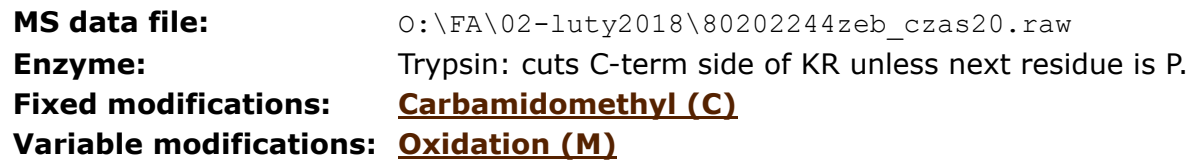

\section{Protein sequence coverage: $\mathbf{3 0} \%$}

Matched peptides shown in bold red.

1 MAKVTLTIKN GNVKESQQFE IDKITTFQAL KLKNEIHAIL KDLKNNGELK

51 EVMEGLFSGE FDVDNMDIKN ITADQLEQMK DEKFITSLAG AFDRLLETVP 101 ERAMNLLSIM SGIDREVLEK AYLEELFDVY DAVMEENDII KLIDRMKRSF 151 FTTKGQWSQA LRTFLANK

Unformatted sequence string: $\mathbf{1 6 8}$ residues (for pasting into other applications).

Sort peptides by $\bigcirc$ Residue Number $\bigcirc$ Increasing Mass $\bigcirc$ Decreasing Mass

Show predicted peptides also

\begin{tabular}{|c|c|c|c|c|}
\hline Query & Start - End & Observed & $\operatorname{Mr}(\exp t)$ & $\operatorname{Mr}(\mathrm{calc})$ \\
\hline 4826 & $24-31$ & 461.2764 & 920.5382 & 920.5331 \\
\hline$\nabla^{4} \underline{8827}$ & $24-31$ & 461.2767 & 920.5388 & 920.5331 \\
\hline$\checkmark 14323$ & $32-41$ & 393.5820 & 1177.7241 & 1177.7182 \\
\hline$\sigma^{\prime 14324}$ & $32-41$ & 393.5821 & 1177.7244 & 1177.7182 \\
\hline$\checkmark 14325$ & $32-41$ & 589.8707 & 1177.7269 & 1177.7182 \\
\hline$\checkmark 14326$ & $32-41$ & 589.8708 & 1177.7270 & 1177.7182 \\
\hline$\checkmark 30287$ & $70-83$ & 831.9073 & 1661.8000 & 1661.7930 \\
\hline$\checkmark \underline{30288}$ & $70-83$ & 554.9407 & 1661.8002 & 1661.7930 \\
\hline 30289 & $70-83$ & 554.9409 & 1661.8009 & 1661.7930 \\
\hline$\checkmark 30290$ & $70-83$ & 831.9080 & 1661.8014 & 1661.7930 \\
\hline$\checkmark 30291$ & $70-83$ & 831.9080 & 1661.8015 & 1661.7930 \\
\hline 30292 & $70-83$ & 554.9413 & 1661.8020 & 1661.7930 \\
\hline$\nearrow^{\prime 30293}$ & $70-83$ & 554.9414 & 1661.8023 & 1661.7930 \\
\hline$\checkmark 30294$ & $70-83$ & 554.9415 & 1661.8026 & 1661.7930 \\
\hline$\nearrow^{\prime 30295}$ & $70-83$ & 554.9419 & 1661.8037 & 1661.7930 \\
\hline$\square^{\prime 15079}$ & $84-94$ & 599.3177 & 1196.6208 & 1196.6190 \\
\hline$\checkmark \underline{6049}$ & $95-102$ & 478.7765 & 955.5384 & 955.5338 \\
\hline$\triangle \underline{6050}$ & $95-102$ & 478.7765 & 955.5385 & 955.5338 \\
\hline$\triangle \underline{6051}$ & $95-102$ & 478.7766 & 955.5386 & 955.5338 \\
\hline$\triangle 0 \underline{6052}$ & $95-102$ & 478.7766 & 955.5386 & 955.5338 \\
\hline$\checkmark \underline{6053}$ & $95-102$ & 478.7767 & 955.5388 & 955.5338 \\
\hline$\checkmark \underline{6055}$ & $95-102$ & 478.7768 & 955.5391 & 955.5338 \\
\hline$\triangle \underline{6056}$ & $95-102$ & 478.7770 & 955.5395 & 955.5338 \\
\hline$\sigma^{6058}$ & $95-102$ & 478.7774 & 955.5403 & 955.5338 \\
\hline$\nabla^{6059}$ & $95-102$ & 478.7776 & 955.5406 & 955.5338 \\
\hline
\end{tabular}

$\begin{array}{llr}\text { ppm } & \text { M } & \text { Score } \\ 5.49 & 0 & 57 \\ 6.21 & 0 & 62 \\ 4.96 & 1 & 63 \\ 5.26 & 1 & 60 \\ 7.32 & 1 & 82 \\ 7.47 & 1 & 80 \\ 4.20 & 1 & 126 \\ 4.33 & 1 & 71 \\ 4.76 & 1 & 63 \\ 5.04 & 1 & 120 \\ 5.10 & 1 & 120 \\ 5.41 & 1 & 75 \\ 5.58 & 1 & 68 \\ 5.79 & 1 & 65 \\ 6.44 & 1 & 70 \\ 1.55 & 0 & 72 \\ 4.81 & 0 & 56 \\ 4.88 & 0 & 56 \\ 4.98 & 0 & 59 \\ 5.02 & 0 & 63 \\ 5.23 & 0 & 56 \\ 5.52 & 0 & 51 \\ 5.90 & 0 & 60 \\ 6.74 & 0 & 60 \\ 7.05 & 0 & 60\end{array}$

$\begin{array}{rc}\text { Expect } & \text { Ran } \\ 2.1 e-06 & 1 \\ 6.6 e-07 & 1 \\ 5.6 e-07 & -1 \\ 9.7 e-07 & -1 \\ 6.7 e-09 & -1 \\ 9.9 e-09 & -1 \\ 2.5 e-13 & -1 \\ 8.7 e-08 & -1 \\ 4.8 e-07 & -1 \\ 9.4 e-13 & -1 \\ 9.1 e-13 & 1 \\ 3 e-08 & 1 \\ 1.6 e-07 & -1 \\ 2.9 e-07 & -1 \\ 9.7 e-08 & 1 \\ 5.9 e-08 & -1 \\ 2.4 e-06 & -1 \\ 2.2 e-06 & -1 \\ 1.1 e-06 & -1 \\ 4.7 e-07 & -1 \\ 2.5 e-06 & -1 \\ 8.1 e-06 & -1 \\ 1.1 e-06 & -1 \\ 1.1 e-06 & -1 \\ 1.1 e-06 & -1 \\ & \end{array}$

U Peptide

K. ITTFQALK. I

U K. ITTFOALK. L

U K. LKNEIHAILK.D

U K. LKNEIHAILK.D

U K. LKNEIHAILK.D

U K. LKNEIHAILK.D

U K. NITADQLEQMKDEK. F

U K. NITADQLEQMKDEK. F

U K.NITADQLEQMKDEK. F

U K.NITADQLEQMKDEK. F

U K.NITADQLEQMKDEK. F

U K.NITADQLEQMKDEK. F

U K.NITADQLEQMKDEK.F

U K. NITADQLEQMKDEK. F

U K.NITADQLEQMKDEK.F

U K. FITSLAGAFDR.L

U R. LLETVPER. A

U R. LLETVPER. A

U R. LLETVPER. A

U R. LLETVPER. A

U R. LLETVPER. A

U R. LLETVPER. A

U R. LLETVPER.A

U R. LLETVPER. A

U R. LLETVPER. A

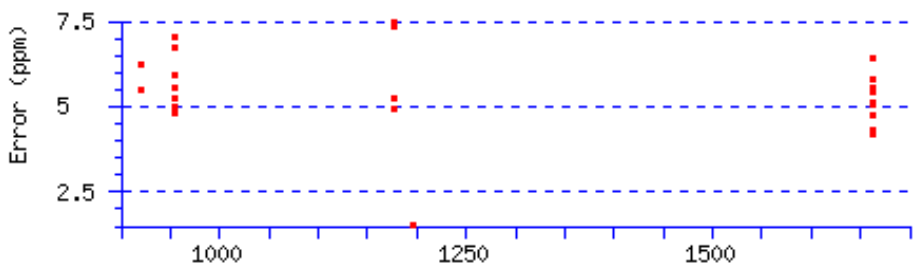

RMS error 5 ppm

Mass (Da) 


\title{
MASCOT Search Results
}

\section{Protein View: TP84_19}

\author{
Database: TP84 \\ Score: 2591 \\ Nominal mass $\left(\mathbf{M}_{\mathbf{r}}\right)$ : 19335 \\ Calculated pI: $\quad 4.90$
}

Sequence similarity is available as an NCBI BLAST search of 74. against nr.

\section{Search parameters}

MS data file:

Enzyme:

Fixed modifications:

Variable modifications:
0: \FA $\backslash 02-1$ uty2018\80202245zeb_Czas25.raw

Trypsin: cuts C-term side of KR unless next residue is $\mathrm{P}$.

Carbamidomethyl (C)

\section{Protein sequence coverage: $60 \%$}

Matched peptides shown in bold red.

1 MAKVTLTIKN GNVKESQQFE IDKITTFQAL KLKNEIHAIL KDLKNNGELK

51 EVMEGLFSGE FDVDNMDIKN ITADQLEQMK DEKFITSLAG AFDRLLETVP 101 ERAMNLLSIM SGIDREVLEK AYLEELFDVY DAVMEENDII KLIDRMKRSF 151 FTTKGQWSQA LRTFLANK

Unformatted sequence string: 168 residues (for pasting into other applications).

Sort peptides by $\odot$ Residue Number $\bigcirc$ Increasing Mass $\bigcirc$ Decreasing Mass

Show predicted peptides also

\begin{tabular}{|c|c|}
\hline Query & Start - End \\
\hline$\varangle 7571$ & $15-23$ \\
\hline$\widetilde{7572}$ & $15-23$ \\
\hline$\checkmark 7573$ & $15-23$ \\
\hline$\longdiv { 7 5 7 4 }$ & $15-23$ \\
\hline$\checkmark 7575$ & $15-23$ \\
\hline ‘7576 & $15-23$ \\
\hline$\widetilde{7577}$ & $15-23$ \\
\hline$\checkmark \underline{3031}$ & $24-31$ \\
\hline$\checkmark x 9019$ & $32-41$ \\
\hline$\overline{9020}$ & $32-41$ \\
\hline$\checkmark 09021$ & $32-41$ \\
\hline$\overline{9022}$ & $32-41$ \\
\hline$\checkmark 9023$ & $32-41$ \\
\hline$\overline{9024}$ & $32-41$ \\
\hline$\checkmark 9025$ & $32-41$ \\
\hline$\checkmark \underline{9027}$ & $32-41$ \\
\hline$\overline{9028}$ & $32-41$ \\
\hline$\checkmark \underline{9029}$ & $32-41$ \\
\hline$\checkmark 9031$ & $32-41$ \\
\hline$\checkmark 9032$ & $32-41$ \\
\hline$\checkmark 9033$ & $32-41$ \\
\hline$\checkmark \underline{3352}$ & $34-41$ \\
\hline$\checkmark 2 \overline{1344}$ & $70-83$ \\
\hline$\checkmark 21345$ & $70-83$ \\
\hline$\overline{21346}$ & $70-83$ \\
\hline$\checkmark 21347$ & $70-83$ \\
\hline$\checkmark \overline{21348}$ & $70-83$ \\
\hline$\checkmark 9549$ & $84-94$ \\
\hline$\checkmark \underline{9550}$ & $84-94$ \\
\hline$\checkmark \underline{9551}$ & $84-94$ \\
\hline$\checkmark \underline{9552}$ & $84-94$ \\
\hline$\checkmark 9553$ & $84-94$ \\
\hline$\checkmark \underline{9555}$ & $84-94$ \\
\hline$\checkmark 9556$ & $84-94$ \\
\hline$\varangle \underline{9557}$ & $84-94$ \\
\hline$\overline{3857}$ & $95-102$ \\
\hline$\checkmark \underline{3858}$ & $95-102$ \\
\hline$\checkmark 3859$ & $95-102$ \\
\hline$\checkmark 3860$ & $95-102$ \\
\hline$\sigma^{\prime 15477}$ & $103-115$ \\
\hline$\checkmark 15478$ & $103-115$ \\
\hline
\end{tabular}

\begin{tabular}{|c|c|c|c|c|}
\hline ppm & $\mathbf{M}$ & Score & Expect & Ran] \\
\hline-0.64 & $\dddot{0}$ & 57 & $1.8 e-06$ & 1 \\
\hline-0.58 & 0 & 53 & $4.6 e-06$ & 1 \\
\hline-0.39 & 0 & 57 & $1.9 e-06$ & 1 \\
\hline-0.26 & 0 & 62 & $6.8 e-07$ & \\
\hline 0.54 & 0 & 73 & $5.2 e-08$ & 1 \\
\hline 0.88 & 0 & 59 & $1.2 e-06$ & \\
\hline 1.63 & 0 & 67 & $2.2 e-07$ & \\
\hline-1.13 & 0 & 62 & $6.4 e-07$ & \\
\hline-1.69 & 1 & 79 & 1. $3 e-08$ & \\
\hline-1.39 & 1 & 71 & $8.6 e-08$ & \\
\hline-1.21 & 1 & 53 & $5.4 e-06$ & $\ldots$ \\
\hline-1.06 & 1 & 63 & $5.5 e-07$ & \\
\hline-0.95 & 1 & 71 & $8.7 e-08$ & 1 \\
\hline-0.95 & 1 & 62 & $5.6 e-07$ & "̈ \\
\hline-0.83 & 1 & 65 & $3.5 e-07$ & 1 \\
\hline 0.063 & 1 & 68 & $1.7 e-07$ & 1 \\
\hline 0.012 & 1 & 65 & $2.9 e-07$ & 1 \\
\hline 0.48 & 1 & 91 & $8.2 e-10$ & $\cdots$ \\
\hline 0.97 & 1 & 101 & $7.6 e-11$ & 1 \\
\hline 1.04 & 1 & 80 & $9.4 e-09$ & $\ldots$ \\
\hline 1.11 & 1 & 98 & 1. $6 e-10$ & 1 \\
\hline 0.083 & 0 & 59 & $1.3 e-06$ & 1 \\
\hline-0.23 & 1 & 91 & $8.8 e-10$ & 1 \\
\hline 0.63 & 1 & 73 & $5.1 e-08$ & 1 \\
\hline 0.90 & 1 & 61 & $7.8 e-07$ & 1 \\
\hline 1.05 & 1 & 72 & $6 e-08$ & 1 \\
\hline 1.98 & 1 & 69 & $1.2 e-07$ & 1 \\
\hline-0.79 & 0 & 70 & $9.4 e-08$ & 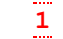 \\
\hline-0.34 & 0 & 73 & $4.9 e-08$ & 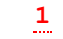 \\
\hline-0.25 & 0 & 72 & $5.7 e-08$ & 1 \\
\hline 0.054 & 0 & 67 & $2.1 e-07$ & $\therefore$ \\
\hline 0.013 & 0 & 71 & $7.2 e-08$ & 1 \\
\hline 0.029 & 0 & 76 & $2.4 e-08$ & 1 \\
\hline 0.11 & 0 & 72 & $6.7 e-08$ & \\
\hline 0.31 & 0 & 83 & $5.6 e-09$ & 1 \\
\hline-0.19 & 0 & 64 & $4.3 e-07$ & $\cdots$ \\
\hline 0.59 & 0 & 63 & $4.7 e-07$ & 1 \\
\hline 0.75 & 0 & 61 & $8.4 e-07$ & 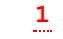 \\
\hline 0.82 & 0 & 60 & $1.1 e-06$ & 1 \\
\hline 0.019 & 0 & 103 & $4.8 e-11$ & \\
\hline 3.21 & 0 & 86 & $2.8 e-09$ & \\
\hline
\end{tabular}

U Peptide

Ü K.ESQQFEIDK. I

U K.ESQQFEIDK. I

U K. ESQQFEIDK. I

U K. ESQQFEIDK. I

U K.ESQQFEIDK. I

U K.ESQQFEIDK.I

U K.ESQQFEIDK.I

U K. ITTFOALK. L

U K. LKNEIHAILK.D

U K. LKNEIHAILK. D

U K. LKNEIHAILK. D

U K. LKNEIHAILK. D

U K. LKNEIHAILK.D

U K. LKNE IHAILK. D

U K. LKNEIHAILK.D

U K. LKNEIHAILK. D

U K. LKNEIHAILK.D

U K. LKNEIHAILK.D

U K. LKNEIHAILK.D

U K. LKNEIHAILK. D

U K. LKNEIHAILK. D

U K.NEIHAILK.D

U K.NITADQLEQMKDEK.F

U K. NITADQLEQMKDEK.F

U K. NITADQLEQMKDEK.F

U K.NITADQLEQMKDEK.F

U K.NITADQLEQMKDEK.F

U K.FITSLAGAFDR.L

U K.FITSLAGAFDR. L

U K.FITSLAGAFDR.L

U K. FITSLAGAFDR. L

U K.FITSLAGAFDR.L

U K. FITSLAGAFDR. L

U K.FITSLAGAFDR.L

U K.FITSLAGAFDR.L

U R. LLETVPER. A

U R. LLETVPER. A

U R. LLETVPER. A

U R. LLETVPER. A

U R. AMNLLSIMSGIDR.E

U R. AMNLLSIMSGIDR.E 
Query Start - End

$121-141$

$121-141$

$\checkmark 3573 \quad 155-162$
Observed Mr (expt) Mr (calc)

$840.4017 \quad 2518.1831 \quad 2518.1825$

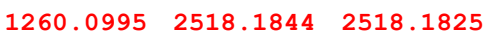

$473.2491 \quad 944.4836 \quad 944.4828$ ppm M Score

$0.250 \quad 75$

$0.780 \quad 120$

$0.830 \quad 54$
Expect Rank U Peptide

3.3e-08 1 U K.AYLEELFDVYDAVMEENDIIK.L

$9.6 e-13 \quad 1 \quad$ U K.AYLEELFDVYDAVMEENDIIK. L

4. $4 \mathrm{e}-06 \quad 1$ U K.GQWSQALR.T

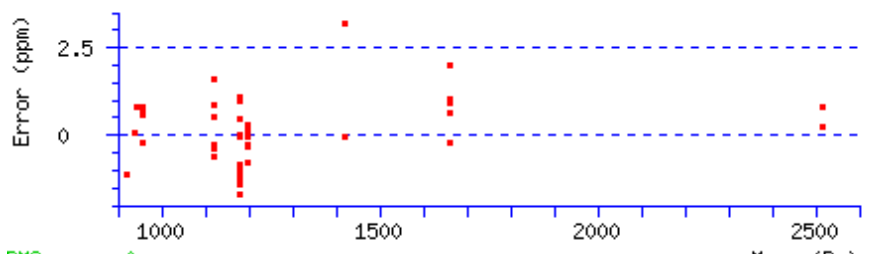

RMS error 0 ppm Mass (Da) 


\title{
MASCOT Search Results
}

\section{Protein View: TP84_19}

\author{
Database: TP84 \\ Score: $\quad 5197$ \\ Nominal mass $\left(\mathbf{M}_{\mathbf{r}}\right): 19335$ \\ Calculated pI: $\quad 4.90$
}

Sequence similarity is available as an NCBI BLAST search of 74. against nr.

\section{Search parameters}

MS data file:

Enzyme:

Fixed modifications:

Variable modifications:
0:\FA 02-luty2018\80202246zeb_Czas30.raw

Trypsin: cuts C-term side of KR unless next residue is $\mathrm{P}$.

Carbamidomethyl (C)

Oxidation (M)

\section{Protein sequence coverage: $55 \%$}

Matched peptides shown in bold red.

1 MAKVTLTIKN GNVKESQQFE IDKITTFQAL KLKNEIHAIL KDLKNNGELK

51 EVMEGLFSGE FDVDNMDIKN ITADQLEQMK DEKFITSLAG AFDRLLETVP 101 ERAMNLISIM SGIDREVLEK AYLEELFDVY DAVMEENDII KLIDRMKRSF 151 FTTKGQWSQA LRTFLANK

Unformatted sequence string: $\mathbf{1 6 8}$ residues (for pasting into other applications).

Sort peptides by $\odot$ Residue Number $\bigcirc$ Increasing Mass $\bigcirc$ Decreasing Mass

Show predicted peptides also

\begin{tabular}{|c|c|}
\hline Query & Start - End \\
\hline$\checkmark 10395$ & $15-23$ \\
\hline$\checkmark 10396$ & $15-23$ \\
\hline$\checkmark 10397$ & $15-23$ \\
\hline$\varpi^{3847}$ & $24-31$ \\
\hline$\overline{3848}$ & $24-31$ \\
\hline$\checkmark 3849$ & $24-31$ \\
\hline$\checkmark 3853$ & $24-31$ \\
\hline$\overline{3856}$ & $24-31$ \\
\hline$\searrow^{3859}$ & $24-31$ \\
\hline$\nabla^{\prime 12662}$ & $32-41$ \\
\hline$\nearrow^{\prime 12663}$ & $32-41$ \\
\hline$ه 12664$ & $32-41$ \\
\hline$\nearrow^{\prime 12665}$ & $32-41$ \\
\hline${ }^{\prime 12666}$ & $32-41$ \\
\hline$\triangle 12667$ & $32-41$ \\
\hline$\triangle 12668$ & $32-41$ \\
\hline$ð 12669$ & $32-41$ \\
\hline$\triangle 12670$ & $32-41$ \\
\hline$\triangle 12671$ & $32-41$ \\
\hline$\triangle 12672$ & $32-41$ \\
\hline$\checkmark 29164$ & $70-83$ \\
\hline$\checkmark 29165$ & $70-83$ \\
\hline$\checkmark 29166$ & $70-83$ \\
\hline$\triangleleft^{\prime 29167}$ & $70-83$ \\
\hline$\nearrow^{\prime 29168}$ & $70-83$ \\
\hline$\nearrow^{29169}$ & $70-83$ \\
\hline$\varangle \underline{29170}$ & $70-83$ \\
\hline$\checkmark 29171$ & $70-83$ \\
\hline$\varangle \underline{29172}$ & $70-83$ \\
\hline$ð 29173$ & $70-83$ \\
\hline$\varangle 29174$ & $70-83$ \\
\hline$\nearrow^{29175}$ & $70-83$ \\
\hline$\varangle 29176$ & $70-83$ \\
\hline$\varangle 29177$ & $70-83$ \\
\hline$\varangle 29178$ & $70-83$ \\
\hline$\varangle \underline{29179}$ & $70-83$ \\
\hline$\checkmark 29180$ & $70-83$ \\
\hline$ð^{2} 29181$ & $70-83$ \\
\hline$\varangle 29182$ & $70-83$ \\
\hline$ð 29183$ & $70-83$ \\
\hline$\nearrow^{\prime 29184}$ & $70-83$ \\
\hline
\end{tabular}

\begin{tabular}{|c|c|c|c|c|}
\hline ppm & $\mathbf{M}$ & Score & Expect & Rar \\
\hline-1.33 & 0 & 56 & $2.8 e-06$ & 1 \\
\hline-0.74 & 0 & 53 & $4.5 e-06$ & 1 \\
\hline 2.18 & 0 & 67 & $2.2 e-07$ & 1 \\
\hline-0.53 & 0 & 59 & 1. $3 e-06$ & \\
\hline-0.44 & 0 & 62 & $6.2 e-07$ & 1 \\
\hline-0.44 & 0 & 58 & $1.7 e-06$ & \\
\hline-0.29 & 0 & 58 & $1.7 e-06$ & \\
\hline 0.039 & 0 & 58 & $1.8 e-06$ & \\
\hline 0.23 & 0 & 56 & $2.6 e-06$ & L \\
\hline-1.77 & 1 & 73 & $5.3 e-08$ & \\
\hline-1.72 & 1 & 63 & $5.4 e-07$ & 1 \\
\hline-1.29 & 1 & 67 & $1.9 e-07$ & \\
\hline-0.70 & 1 & 69 & $1.3 e-07$ & 1 \\
\hline-0.55 & 1 & 66 & $2.8 e-07$ & L \\
\hline 0.22 & 1 & 83 & $5.5 e-09$ & \\
\hline 0.46 & 1 & 83 & $5.6 e-09$ & 1 \\
\hline 0.99 & 1 & 89 & $1.4 e-09$ & \\
\hline 1.00 & 1 & 83 & $5.4 e-09$ & 1 \\
\hline 1.63 & 1 & 83 & $5.5 e-09$ & \\
\hline 1.70 & 1 & 69 & $1.2 e-07$ & 1 \\
\hline-2.16 & 1 & 114 & 3. $9 e-12$ & \\
\hline-0.71 & 1 & 112 & $6.8 e-12$ & 1 \\
\hline-0.43 & 1 & 114 & $3.8 e-12$ & \\
\hline-0.43 & 1 & 112 & $6.8 e-12$ & 1 \\
\hline-0.40 & 1 & 124 & $4.5 e-13$ & 1 \\
\hline-0.34 & 1 & 115 & $3.5 e-12$ & 1 \\
\hline-0.23 & 1 & 121 & $8.7 e-13$ & 1 \\
\hline-0.18 & 1 & 69 & $1.1 e-07$ & 1 \\
\hline 0.088 & 1 & 120 & $9.1 e-13$ & 1 \\
\hline 0.070 & 1 & 65 & $3.2 e-07$ & \\
\hline 0.32 & 1 & 72 & $6.1 e-08$ & 1 \\
\hline 0.38 & 1 & 84 & $3.8 e-09$ & 1. \\
\hline 0.59 & 1 & 70 & $1 e-07$ & 1 \\
\hline 0.77 & 1 & 114 & 3. $7 e-12$ & 1 \\
\hline 0.81 & 1 & 117 & 1. $9 e-12$ & 1 \\
\hline 0.99 & 1 & 95 & 3. $3 e-10$ & 1 \\
\hline 1.03 & 1 & 65 & 2. $9 e-07$ & 1 \\
\hline 1.44 & 1 & 70 & $9.7 e-08$ & 1 \\
\hline 1.53 & 1 & 73 & $4.8 e-08$ & 1 \\
\hline 1.62 & 1 & 69 & $1.2 e-07$ & 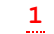 \\
\hline 2.00 & 1 & 64 & $4.1 e-07$ & \\
\hline
\end{tabular}

U Peptide

Ü K.ESQQFEIDK. I

U K.ESQQFEIDK. I

U K.ESQQFEIDK.I

U K. ITTFQALK. L

U K. ITTFQALK. L

U K. ITTFOALK. L

U K. ITTFQALK. L

U K. ITTFQALK. L

U K. ITTFQALK. L

U K. LKNEIHAILK. D

U K. LKNEIHAILK.D

U K. LKNEIHAILK. D

U K. LKNEIHAILK.D

U K. LKNEIHAILK.D

U K. LKNEIHAILK.D

U K. LKNEIHAILK. D

U K. LKNEIHAILK.D

U K. LKNEIHAILK. D

U K. LKNEIHAILK.D

U K. LKNEIHAILK. D

U K.NITADQLEQMKDEK.F

U K.NITADQLEQMKDEK.F

U K. NITADQLEQMKDEK.F

U K.NITADQLEQMKDEK.F

U K. NITADQLEQMKDEK. F

U K.NITADQLEQMKDEK.F

U K. NITADQLEQMKDEK.F

U K.NITADQLEQMKDEK.F

U K. NITADQLEQMKDEK. F

U K. NITADQLEQMKDEK.F

U K. NITADQLEQMKDEK.F

U K.NITADQLEQMKDEK.F

U K . NITADQLEOMKDEK . F

U K. NITADQLEQMKDEK.F

U K. NITADQLEQMKDEK. F

U K.NITADQLEQMKDEK.F

U K. NITADOLEOMKDEK . F

U K.NITADQLEQMKDEK.F

U K.NITADQLEQMKDEK.F

U K.NITADQLEQMKDEK.F

$U$ K. NITADQLEQMKDEK.F 
Query Start - End

$\checkmark 13401$

$\square 13402 \quad 84-94$

đ13403 $84-94$

$\square 13404 \quad 84-94$

$\square 13405 \quad 84-94$

$813406 \quad 84-94$

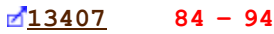

$\square 13408 \quad 84-94$

$813409 \quad 84-94$

$\square 13410 \quad 84-94$

$813411 \quad 84-94$

$\square 13412 \quad 84-94$

$13413 \quad 84-94$

$\square 13414 \quad 84-94$

$\triangle 13415 \quad 84-94$

$\square 13416 \quad 84-94$

$\square 13417 \quad 84-94$

$\square 4890 \quad 95-102$

$\checkmark \underline{4891} \quad 95-102$

$\varangle 4892 \quad 95-102$

$\checkmark \underline{4893} \quad 95-102$

$\triangle 4894 \quad 95-102$

$\checkmark \underline{4895} \quad 95-102$

$\checkmark 4 \mathbf{4 8 9 7} 95-102$

$\checkmark 4 \underline{488} \quad 95-102$

$\checkmark 4899 \quad 95-102$

$\square \underline{4900} \quad 95-102$

$\varangle 4901 \quad 95-102$

$\varangle \underline{4902} \quad 95-102$

$\varangle 21829 \quad 103-115$

$\varangle 21830 \quad 103-115$

$\checkmark 21832 \quad 103-115$

$\varangle 21833 \quad 103-115$

$021834 \quad 103-115$

$\varangle \underline{21835} \quad 103-115$

$\checkmark 21836 \quad 103-115$

$\checkmark 43565 \quad 121-141$

$\checkmark \underline{43566} \quad 121-141$
Observed $\quad \mathrm{Mr}$ (expt) $\quad \mathrm{Mr}$ (calc)

$\begin{array}{llll}599.3166 & 1196.6187 & 1196.6190\end{array}$

$599.3170 \quad 1196.6195 \quad 1196.6190$

$599.3171 \quad 1196.6196 \quad 1196.6190$

$599.3171 \quad 1196.6196 \quad 1196.6190$

$\begin{array}{lll}599.3172 & 1196.6198 & 1196.6190\end{array}$

$\begin{array}{lll}599.3172 & 1196.6198 & 1196.6190\end{array}$

$\begin{array}{lll}599.3173 & 1196.6200 & 1196.6190\end{array}$

$\begin{array}{llll}599.3173 & 1196.6200 & 1196.6190\end{array}$

$\begin{array}{llll}599.3173 & 1196.6200 & 1196.6190\end{array}$

$\begin{array}{llll}599.3174 & 1196.6202 & 1196.6190\end{array}$

$\begin{array}{lll}599.3174 & 1196.6202 & 1196.6190\end{array}$

$599.3175 \quad 1196.6204 \quad 1196.6190$

$\begin{array}{llll}599.3175 & 1196.6204 & 1196.6190\end{array}$

$599.3175 \quad 1196.6205 \quad 1196.6190$

$599.3177 \quad 1196.6208 \quad 1196.6190$

$\begin{array}{llll}599.3177 & 1196.6208 & 1196.6190\end{array}$

$\begin{array}{lll}599.3179 & 1196.6212 & 1196.6190\end{array}$

$\begin{array}{lll}478.7738 & 955.5330 & 955.5338\end{array}$

$\begin{array}{lll}478.7740 & 955.5333 & 955.5338\end{array}$

$\begin{array}{lll}478.7742 & 955.5338 & 955.5338\end{array}$

$\begin{array}{lll}478.7743 & 955.5340 & 955.5338\end{array}$

$\begin{array}{lll}478.7743 & 955.5340 & 955.5338\end{array}$

$\begin{array}{lll}478.7743 & 955.5341 & 955.5338\end{array}$

$\begin{array}{lll}478.7745 & 955.5344 & 955.5338\end{array}$

$\begin{array}{lll}478.7745 & 955.5344 & 955.5338\end{array}$

$\begin{array}{lll}478.7745 & 955.5344 & 955.5338\end{array}$

$\begin{array}{lll}478.7746 & 955.5347 & 955.5338\end{array}$

$\begin{array}{lll}478.7747 & 955.5349 & 955.5338\end{array}$

$\begin{array}{lll}478.7749 & 955.5352 & 955.5338\end{array}$

$\begin{array}{lll}710.8675 & 1419.7205 & 1419.7214\end{array}$

$\begin{array}{llll}710.8677 & 1419.7207 & 1419.7214\end{array}$

$\begin{array}{lll}710.8678 & 1419.7210 & 1419.7214\end{array}$

$\begin{array}{lll}710.8678 & 1419.7211 & 1419.7214\end{array}$

$\begin{array}{lll}710.8683 & 1419.7220 & 1419.7214\end{array}$

$\begin{array}{lll}710.8689 & 1419.7233 & 1419.7214\end{array}$

$\begin{array}{lll}710.8691 & 1419.7236 & 1419.7214\end{array}$

$\begin{array}{lll}1260.0964 & 2518.1782 & 2518.1825\end{array}$

$\begin{array}{rr}840.4011 & 2518.1816 \quad 2518.1825\end{array}$

\begin{tabular}{|c|c|c|c|c|}
\hline ppm & $\mathbf{M}$ & Score & Expect & Ran \\
\hline-0.19 & $\dddot{0}$ & 76 & $2.8 e-08$ & 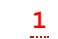 \\
\hline 0.45 & 0 & 82 & $5.9 e-09$ & \\
\hline 0.51 & 0 & 83 & $5.6 e-09$ & \\
\hline 0.55 & 0 & 79 & $1.3 e-08$ & \\
\hline 0.71 & 0 & 73 & $5.6 e-08$ & \\
\hline 0.73 & 0 & 69 & $1.3 e-07$ & \\
\hline 0.85 & 0 & 83 & $4.7 e-09$ & \\
\hline 0.85 & 0 & 71 & $7.6 e-08$ & \\
\hline 0.90 & 0 & 75 & $2.9 e-08$ & \\
\hline 1.00 & 0 & 70 & $9.2 e-08$ & \\
\hline 1.07 & 0 & 88 & $1.6 e-09$ & \\
\hline 1.22 & 0 & 82 & $5.7 e-09$ & \\
\hline 1.23 & 0 & 79 & $1.3 e-08$ & \\
\hline 1.27 & 0 & 82 & $6.1 e-09$ & \\
\hline 1.52 & 0 & 79 & $1.4 e-08$ & \\
\hline 1.57 & 0 & 75 & $2.9 e-08$ & 1 \\
\hline 1.90 & 0 & 84 & $3.5 e-09$ & \\
\hline-0.88 & 0 & 64 & $4.2 e-07$ & 1 \\
\hline-0.50 & 0 & 63 & $4.6 e-07$ & \\
\hline 0.022 & 0 & 56 & $2.3 e-06$ & \\
\hline 0.15 & 0 & 69 & $1.4 e-07$ & \\
\hline 0.17 & 0 & 69 & $1.4 e-07$ & \\
\hline 0.27 & 0 & 60 & $9.5 e-07$ & \\
\hline 0.56 & 0 & 56 & $2.4 e-06$ & \\
\hline 0.61 & 0 & 59 & $1.2 e-06$ & \\
\hline 0.63 & 0 & 60 & $1.1 e-06$ & \\
\hline 0.92 & 0 & 53 & $5.2 e-06$ & \\
\hline 1.13 & 0 & 54 & $4.3 e-06$ & \\
\hline 1.40 & 0 & 60 & $9.8 e-07$ & \\
\hline-0.62 & 0 & 94 & 3. $9 e-10$ & \\
\hline-0.44 & 0 & 90 & $1 e-09$ & 1 \\
\hline-0.24 & 0 & 92 & $6.7 e-10$ & \\
\hline-0.22 & 0 & 85 & $3 e-09$ & \\
\hline 0.42 & 0 & 81 & $7.8 e-09$ & \\
\hline 1.33 & 0 & 97 & $2 e-10$ & \\
\hline 1.59 & 0 & 100 & 1. $1 e-10$ & \\
\hline-1.68 & 0 & 98 & 1. $7 e-10$ & \\
\hline-0.35 & 0 & 112 & $6.4 e-12$ & \\
\hline
\end{tabular}

U Peptide

U K. FITSLAGAFDR. L

U K.FITSLAGAFDR.L

U K. FITSLAGAFDR. I

U K.FITSLAGAFDR.I

U K. FITSLAGAFDR. I

U K. FITSLAGAFDR. I

U K. FITSLAGAFDR. I

U K.FITSLAGAFDR.I

U K. FITSLAGAFDR. I

U K. FITSLAGAFDR.L

U K. FITSLAGAFDR.L

U K. FITSLAGAFDR. I

U K. FITSLAGAFDR. I

U K.FITSLAGAFDR.I

U K. FITSLAGAFDR. L

U K.FITSLAGAFDR.I

U K. FITSLAGAFDR.L

U R. LLETVPER. A

U R.LLETVPER.A

U R. LLETVPER. A

U R.LLETVPER.A

U R. LLETVPER. A

U R. LLETVPER.A

U R. LLETVPER. A

U R.LLETVPER.A

U R. LLETVPER. A

U R. LLETVPER.A

U R.LLETVPER. A

U R. LLETVPER. A

U R.AMNLISIMSGIDR.E

U R. AMNLLSIMSGIDR.E

U R. AMNLLSIMSGIDR.E

U R. AMNLLSIMSGIDR.E

U R. AMNLLSIMSGIDR.E

U R. AMNLLSIMSGIDR.E

U R. AMNLLSIMSGIDR.E

U K.AYLEELFDVYDAVMEENDIIK. I

$U$ K.AYLEELFDVYDAVMEENDIIK. L
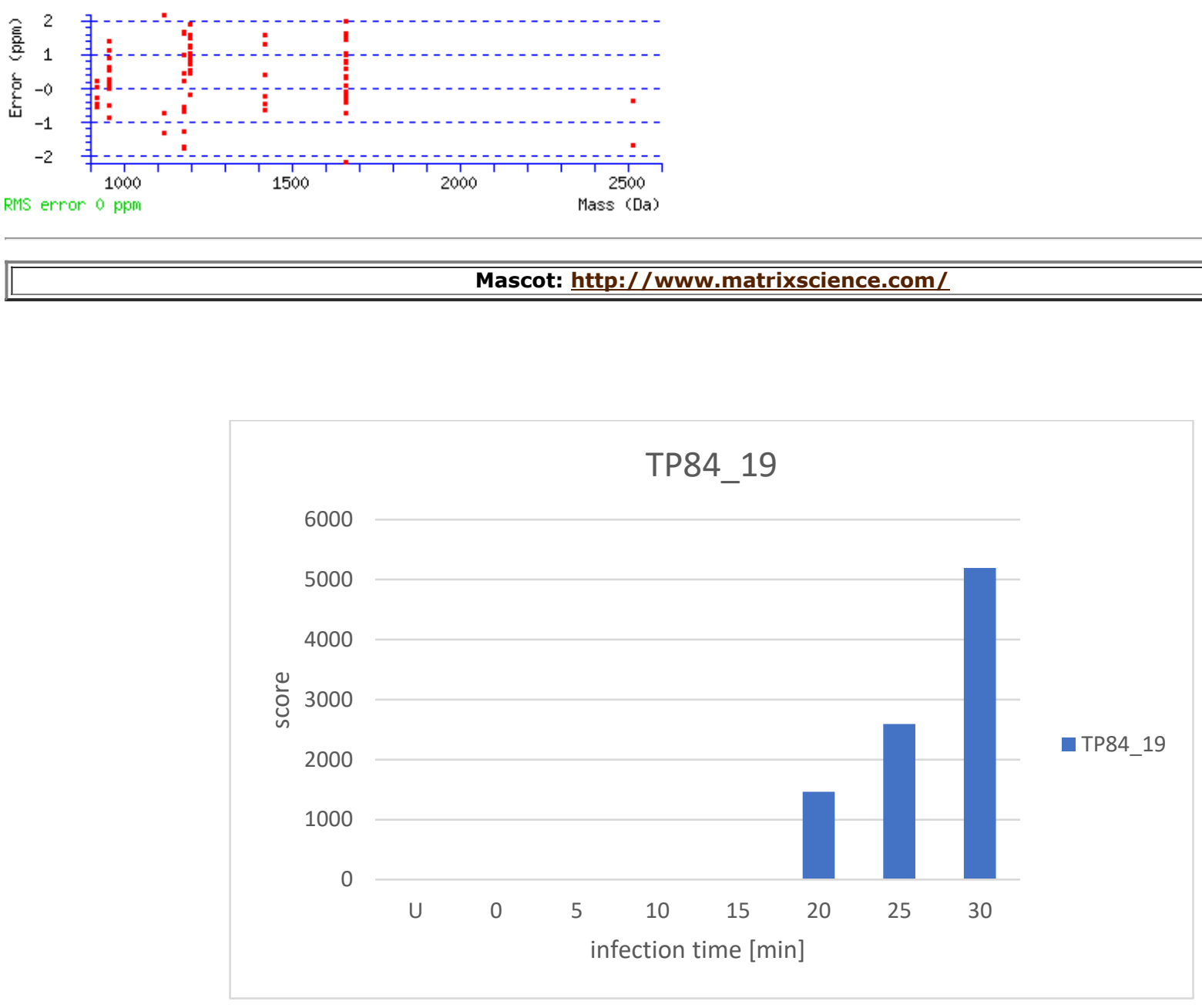


\title{
MASCOT Search Results
}

\section{Protein View: TP84_28}

\author{
Database: $\quad$ TP84 \\ Score: $\quad 1188$ \\ Nominal mass $\left(\mathbf{M}_{\mathbf{r}}\right): 44214$ \\ Calculated pI: $\quad 9.67$
}

Sequence similarity is available as an NCBI BLAST search of 83. against nr.

\section{Search parameters}

\author{
MS data file: $\quad 0: \backslash F A \backslash 02-1 u t y 2018 \backslash 80202245 \mathrm{zeb} c z a s 25$.raw \\ Enzyme: Trypsin: cuts C-term side of KR unless next residue is $P$. \\ Fixed modifications: Carbamidomethyl (C) \\ Variable modifications: Oxidation (M)
}

\section{Protein sequence coverage: $\mathbf{4 1 \%}$}

Matched peptides shown in bold red.

1 MQARSANNIK GIDVSHWQGK IDWAKVKAAG IQVAYLKATE GTTHVDKMLK

51 TNYQNAKKAG IKVGFYHFER AKNEQNAREQ ARHFVNTVKG MPNDLKHALD 101 IETTEGLSNE ALTKCAIAFL EEVKKLTGQD PIVYTYTSFA RSRLTAAIAK 151 YPVWIAHYGV DKPGDNPIWD RWIGFQYTDK GKVSGIAGNV DMNEFTSDIF 201 VDAAKVEQPK QKVDAVQSTP SATGTYTIKS GDTFWELEEK YNWPHGTLQR 251 LNPSVNPNAL KVGQVIKVPK SEQPKQNASS VTGTYTIKSG DTFWDLEQKN 301 GWPHGTLQKL NPGVNPNKLK VGQVIKVPKS EQKNVQRTVK NHQKPNYRTY 351 KIKKGDTFWE LEKKNGWPHG TLQKLNPGVN PAKLQIGQTI KIPN

Unformatted sequence string: $\mathbf{3 9 4}$ residues (for pasting into other applications).

Sort peptides by $\odot$ Residue Number $\bigcirc$ Increasing Mass $\bigcirc$ Decreasing Mass

Show predicted peptides also

\begin{tabular}{|c|c|}
\hline & $r t-E$ \\
\hline$\triangle 7661$ & $11-20$ \\
\hline 27340 & $97-114$ \\
\hline 12 & $115-125$ \\
\hline 58 & $115-125$ \\
\hline 12559 & $115-125$ \\
\hline 12560 & $115-125$ \\
\hline 25281 & $126-141$ \\
\hline 33697 & $151-171$ \\
\hline 33700 & $151-171$ \\
\hline 32981 & $183-205$ \\
\hline 23218 & $213-229$ \\
\hline 14159 & $276-288$ \\
\hline 14160 & $276-288$ \\
\hline$\gamma^{\prime} 14161$ & $276-288$ \\
\hline$\checkmark(3779$ & $310-318$ \\
\hline$\checkmark \underline{3780}$ & $310-318$ \\
\hline 11095 & $354-363$ \\
\hline$\varangle \underline{2831}$ & $375-383$ \\
\hline$\square^{\prime 2} \underline{256}$ & $384-391$ \\
\hline
\end{tabular}

$\begin{array}{rrr}\text { Observed } & \text { Mr(expt) } & \text { Mr (calc) } \\ 563.7873 & 1125.5601 & 1125.5567 \\ 647.9971 & 1940.9695 & 1940.9691 \\ 654.3524 & 1306.6903 & 1306.6955 \\ 436.5714 & 1306.6925 & 1306.6955 \\ 436.5715 & 1306.6925 & 1306.6955 \\ 436.5718 & 1306.6936 & 1306.6955 \\ 916.4665 & 1830.9185 & 1830.9152 \\ 625.3111 & 2497.2154 & 2497.2179 \\ 625.3121 & 2497.2195 & 2497.2179 \\ 1200.5655 & 2399.1164 & 2399.1315 \\ 869.9462 & 1737.8778 & 1737.8785 \\ 685.3525 & 1368.6904 & 1368.6885 \\ 685.3534 & 1368.6922 & 1368.6885 \\ 685.3551 & 1368.6957 & 1368.6885 \\ 476.7648 & 951.5151 & 951.5138 \\ 476.7652 & 951.5159 & 951.5138 \\ 626.8131 & 1251.6117 & 1251.6135 \\ 455.2612 & 908.5079 & 908.5080 \\ 450.7790 & 899.5434 & 899.5440\end{array}$

\begin{tabular}{rrr}
\multicolumn{1}{c}{ Ppm } & M & Score \\
3.03 & 0 & 55 \\
0.24 & 0 & 115 \\
-3.94 & 1 & 68 \\
-2.31 & 1 & 55 \\
-2.26 & 1 & 54 \\
-1.41 & 1 & 54 \\
1.82 & 0 & 86 \\
-0.99 & 0 & 65 \\
0.63 & 0 & 68 \\
-6.27 & 0 & 143 \\
-0.39 & 0 & 106 \\
1.37 & 0 & 90 \\
2.69 & 0 & 90 \\
5.28 & 0 & 83 \\
1.43 & 0 & 52 \\
2.30 & 0 & 56 \\
-1.50 & 1 & 64 \\
-0.090 & 0 & 66 \\
-0.71 & 0 & 53
\end{tabular}

Expect Rank U Peptide

3. $2 e-06 \quad 1$

Ü K. GIDVSHWQGK. I

3. $5 e-12$

U K. HALDIETTEGLSNEALTK . C

$1.6 e-07 \quad 1 \quad$ U K. CAIAFLEEVKK.L

3. $5 e-06 \quad 1$ U K. CAIAFLEEVKK. I

$3.7 e-06 \quad \frac{1}{1}$ U K. CAIAFLEEVKK. L

$3.6 e-06 \quad 1$ U K. CAIAFLEEVKK.I

2.5e-09 1 U K. LTGQDPIVYTYTSFAR.S

3. $4 e-07 \quad 1 \quad$ U $K$. YPVWIAHYGVDKPGDNPIWDR.W

$1.7 e-07 \quad 1 \quad U \quad K$. YPVWIAHYGVDKPGDNPIWDR.W

$5.5 e-15 \quad 1 \quad$ U $\mathrm{K}$.VSGIAGNVDMNEFTSDIFVDAAK.V

$2.4 \mathrm{e}-11 \quad 1 \quad \mathrm{U}$ K.VDAVQSTPSATGTYTIK.S

le-09 1 U U K. QNASSVTGTYTIK.S

9.3e-10 1 U K. ONASSVTGTYTIK.S

$5.2 \mathrm{e}-09 \quad \frac{1}{1}$ U K. QNASSVTGTYTIK.S

$5.7 e-06 \quad 1 \quad$ U K. LNPGVNPNK.I

2.8e-06 1 U K. LNPGVNPNK.L

3.9e-07 1 U K. KGDTFWELEK.K

$2.8 e-07 \quad 1$ U K. LNPGVNPAK.L

$\checkmark \longdiv { 2 5 6 5 } \quad 3 8 4 - 3 9 1$

$\begin{array}{lll}450.7790 \quad 899.5434 & 899.5440\end{array}$

U K. LQIGQTIK.I

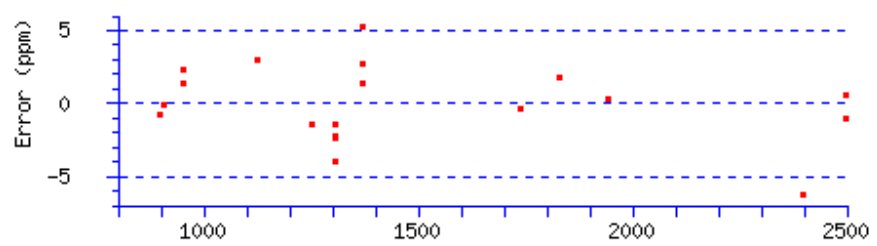




\title{
MASCOT Search Results
}

\section{Protein View: TP84_28}

\author{
Database: TP84 \\ Score: 2309 \\ Nominal mass $\left(\mathbf{M}_{\mathbf{r}}\right): 44214$ \\ Calculated pI: $\quad 9.67$
}

Sequence similarity is available as an NCBI BLAST search of 83. against nr.

\section{Search parameters}

MS data file:

Enzyme:

Fixed modifications:
O: \FA $\backslash 02$-luty2018\80202246zeb czas30.raw

Trypsin: cuts C-term side of $K R$ unless next residue is $P$.

Carbamidomethyl (C)

\section{Protein sequence coverage: $\mathbf{3 7 \%}$}

Matched peptides shown in bold red.

1 MQARSANNIK GIDVSHWQGK IDWAKVKAAG IQVAYLKATE GTTHVDKMLK

51 TNYQNAKKAG IKVGFYHFFR AKNEQNAREQ ARHFVNTVKG MPNDLKHALD 101 IETTEGLSNE ALTKCAIAFL EEVKKLTGQD PIVYTYTSFA RSRLTAAIAK 151 YPVWIAHYGV DKPGDNPIWD RWIGFQYTDK GKVSGIAGNV DMNEFTSDIF 201 VDAAKVEQPK QKVDAVQSTP SATGTYTIKS GDTFWELEEK YNWPHGTLQR 251 LNPSVNPNAL KVGQVIKVPK SEQPKQNASS VTGTYTIKSG DTFWDLEQKN 301 GWPHGTLQKL NPGVNPNKLK VGQVIKVPKS EQKNVQRTVK NHQKPNYRTY 351 KIKKGDTFWE LEKKNGWPHG TLQKLNPGVN PAKLQIGQTI KIPN

Unformatted sequence string: $\mathbf{3 9 4}$ residues (for pasting into other applications).

Sort peptides by $\odot$ Residue Number $\bigcirc$ Increasing Mass $\bigcirc$ Decreasing Mass

Show predicted peptides also

\begin{tabular}{|c|c|}
\hline & $r t-E$ \\
\hline “2192 & $83-89$ \\
\hline$\checkmark \mathbf{2 1 9 3}$ & $83-89$ \\
\hline $3 \overline{6326}$ & $97-114$ \\
\hline 36 & $97-114$ \\
\hline 36328 & $97-114$ \\
\hline 36329 & $97-114$ \\
\hline 36330 & $97-114$ \\
\hline$\underline{0}$ & $115-125$ \\
\hline 33987 & $126-141$ \\
\hline 33988 & $126-141$ \\
\hline 33989 & $126-141$ \\
\hline 43457 & $151-171$ \\
\hline 43459 & $151-171$ \\
\hline 43460 & $51-171$ \\
\hline 61 & $151-171$ \\
\hline 63 & $151-171$ \\
\hline 65 & $151-171$ \\
\hline 43466 & $151-171$ \\
\hline 18 & $183-205$ \\
\hline 42719 & $183-205$ \\
\hline 42720 & $183-205$ \\
\hline$\underline{42721}$ & $183-205$ \\
\hline$\nearrow^{18984}$ & $230-240$ \\
\hline 18985 & $230-240$ \\
\hline$\checkmark 12029$ & $251-261$ \\
\hline$\sigma^{\prime 12030}$ & $251-261$ \\
\hline$\checkmark 12031$ & $251-261$ \\
\hline$\overline{18422}$ & $289-299$ \\
\hline$\nabla^{18424}$ & $289-299$ \\
\hline$\triangle 4770$ & $310-318$ \\
\hline $4 \underline{473}$ & $310-318$ \\
\hline$\sigma^{\prime} \underline{4774}$ & $310-318$ \\
\hline$\checkmark 3341$ & $384-391$ \\
\hline$\sigma^{\prime 3342}$ & $384-391$ \\
\hline 3346 & $384-391$ \\
\hline$\sigma^{\prime} 3350$ & $384-391$ \\
\hline
\end{tabular}

$\begin{array}{rrr}\text { Observed } & \text { Mr (expt) } & \text { Mr (calc) } \\ 422.7376 & 843.4607 & 843.4603 \\ 422.7380 & 843.4614 & 843.4603 \\ 971.4907 & 1940.9669 & 1940.9691 \\ 971.4915 & 1940.9684 & 1940.9691 \\ 971.4916 & 1940.9687 & 1940.9691 \\ 647.9980 & 1940.9721 & 1940.9691 \\ 971.4939 & 1940.9733 & 1940.9691 \\ 436.5717 & 1306.6934 & 1306.6955 \\ 916.4617 & 1830.9088 & 1830.9152 \\ 916.4621 & 1830.9096 & 1830.9152 \\ 916.4636 & 1830.9127 & 1830.9152 \\ 833.4121 & 2497.2146 & 2497.2179 \\ 625.3114 & 2497.2166 & 2497.2179 \\ 625.3116 & 2497.2171 & 2497.2179 \\ 625.3117 & 2497.2178 & 2497.2179 \\ 625.3118 & 2497.2180 & 2497.2179 \\ 625.3119 & 2497.2186 & 2497.2179 \\ 625.3124 & 2497.2205 & 2497.2179 \\ 1200.5717 & 2399.1288 & 2399.1315 \\ 800.7201 & 2399.1383 & 2399.1315 \\ 1200.5771 & 2399.1396 & 2399.1315 \\ 800.7236 & 2399.1491 & 2399.1315 \\ 670.8032 & 1339.5919 & 1339.5932 \\ 670.8035 & 1339.5924 & 1339.5932 \\ 583.8295 & 1165.6445 & 1165.6455 \\ 583.8298 & 1165.6451 & 1165.6455 \\ 583.8300 & 1165.6455 & 1165.6455 \\ 663.3050 & 1324.5955 & 1324.5936 \\ 663.3057 & 1324.5969 & 1324.5936 \\ 476.7645 & 951.5144 & 951.5138 \\ 476.7648 & 951.5150 & 951.5138 \\ 476.7648 & 951.5150 & 951.5138 \\ 450.7789 & 899.5432 & 899.5440 \\ 450.7790 & 899.5434 & 899.5440 \\ 450.7791 & 899.5437 & 899.5440 \\ 450.7795 & 899.5444 & 899.5440\end{array}$

\begin{tabular}{|c|c|c|c|c|}
\hline ppm & M & Score & Expect & Ran \\
\hline 0.51 & $\ddot{0}$ & 59 & $1.4 e-06$ & 1 \\
\hline 1.29 & 0 & 54 & $3.6 e-06$ & 1 \\
\hline-1.13 & 0 & 136 & 2. $3 e-14$ & 1 \\
\hline-0.35 & 0 & 154 & $4.4 e-16$ & 1 \\
\hline-0.18 & 0 & 147 & $2 e-15$ & 1 \\
\hline 1.54 & 0 & 81 & $7.2 e-09$ & 1 \\
\hline 2.17 & 0 & 63 & $4.7 e-07$ & 1 \\
\hline-1.62 & 1 & 56 & $2.7 e-06$ & 1 \\
\hline-3.48 & 0 & 66 & $2.3 e-07$ & 1 \\
\hline-3.07 & 0 & 81 & $8.7 e-09$ & 1 \\
\hline-1.38 & 0 & 75 & $3.2 e-08$ & 1 \\
\hline-1.33 & 0 & 111 & $7.3 e-12$ & 1 \\
\hline-0.51 & 0 & 56 & $2.3 e-06$ & 1 \\
\hline-0.31 & 0 & 61 & $7.4 e-07$ & 1 \\
\hline 0.042 & 0 & 70 & $9 e-08$ & 1 \\
\hline 0.038 & 0 & 62 & $6 e-07$ & 1 \\
\hline 0.28 & 0 & 67 & $2 e-07$ & 1 \\
\hline 1.05 & 0 & 66 & 2. $3 e-07$ & 1 \\
\hline-1.11 & 0 & 134 & $4.1 e-14$ & 1 \\
\hline 2.84 & 0 & 87 & $1.9 e-09$ & 1 \\
\hline 3.40 & 0 & 130 & 1. $1 e-13$ & 1 \\
\hline 7.32 & 0 & 93 & $5.3 e-10$ & 1 \\
\hline-0.97 & 0 & 66 & $2.7 e-07$ & 1 \\
\hline-0.61 & 0 & 79 & $1.3 e-08$ & 1 \\
\hline-0.83 & 0 & 55 & $3.2 e-06$ & 1 \\
\hline-0.31 & 0 & 51 & $7.9 e-06$ & 1 \\
\hline 0.021 & 0 & 54 & $4.3 e-06$ & 1 \\
\hline 1.48 & 0 & 72 & $5.6 e-08$ & 1 \\
\hline 2.52 & 0 & 76 & $2.5 e-08$ & 1 \\
\hline 0.64 & 0 & 64 & $4.3 e-07$ & 1 \\
\hline 1.31 & 0 & 67 & $1.9 e-07$ & 1 \\
\hline 1.33 & 0 & 63 & $5 e-07$ & 1 \\
\hline-0.89 & 0 & 51 & $7.4 e-06$ & 1 \\
\hline-0.64 & 0 & 51 & $8.6 e-06$ & 1 \\
\hline-0.38 & 0 & 54 & $4.4 e-06$ & 1 \\
\hline 0.42 & 0 & 51 & $8.9 e-06$ & 1 \\
\hline
\end{tabular}

U Peptide

U R.HFVNTVK.G

U R. HFVNTVK .G

U K. HALDIETTEGLSNEALTK.C

U K. HALDIETTEGLSNEALTK. C

U K. HALDIETTEGLSNEALTK. C

U K. HALDIETTEGLSNEALTK. C

U K. HALDIETTEGLSNEALTK. C

U K. CAIAFLEEVKK. I

U K. LTGQDPIVYTYTSFAR.S

U K. LTGQDPIVYTYTSFAR.S

U K. LTGODPIVYTYTSFAR.S

U K. YPVWIAHYGVDKPGDNPIWDR.W

U K. YPVWIAHYGVDKPGDNPIWDR. W

U K. YPVWIAHYGVDKPGDNPIWDR.W

U K. YPVWIAHYGVDKPGDNPIWDR.W

U K. YPVWIAHYGVDKPGDNPIWDR.W

U K. YPVWIAHYGVDKPGDNPIWDR. W

U K. YPVWIAHYGVDKPGDNPIWDR.W

U K.VSGIAGNVDMNEFTSDIFVDAAK.V

U K.VSGIAGNVDMNEFTSDIFVDAAK. V

U K.VSGIAGNVDMNEFTSDIFVDAAK.V

U $K$. VSGIAGNVDMNEFTSDIFVDAAK. V

U K.SGDTFWELEEK.Y

U K.SGDTFWELEEK. Y

U R. LNPSVNPNALK.V

U R. LNPSVNPNALK.V

U R. LNPSVNPNALK.V

U K. SGDTFWDLEQK. N

U K. SGDTFWDLEOK.N

U K. LNPGVNPNK. L

U K. LNPGVNPNK. I

U K. LNPGVNPNK. L

U K. LOIGOTIK. I

U K. LQIGQTIK.I

U K. LOIGOTIK. I

U K. LQIGQTIK.I 


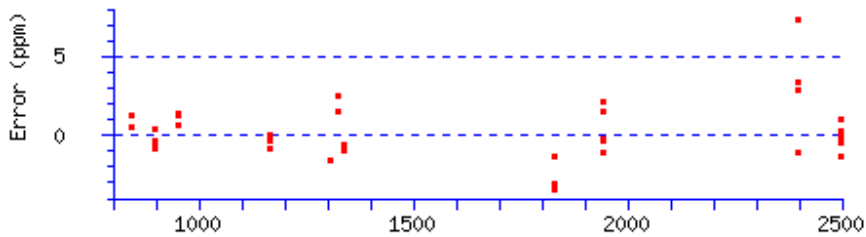
RMS error 1 ppm Mass (Da)

Mascot: http://www.matrixscience.com/

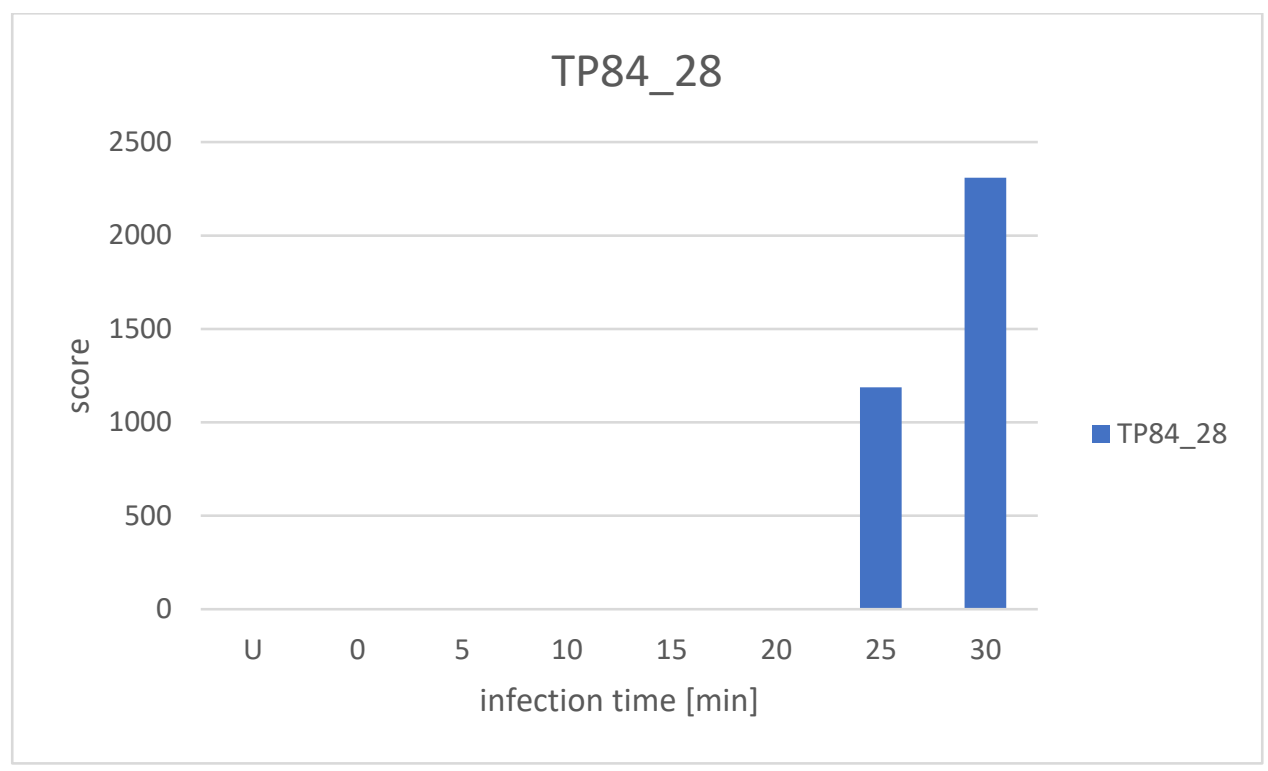




\title{
MASCOT Search Results
}

\section{Protein View: TP84_36}

\author{
Database: TP84 \\ Score: $\quad 440$ \\ Nominal mass ( $\left.\mathbf{M}_{\mathbf{r}}\right)$ : 9310 \\ Calculated pI: $\quad 9.91$
}

Sequence similarity is available as an NCBI BLAST search of 6. against nr.

\section{Search parameters}

\author{
MS data file: \\ $0: \backslash F A \backslash 02-l u t y 2018 \backslash 80202243 z e b$ czas15.raw \\ Enzyme: \\ Trypsin: cuts C-term side of KR unless next residue is $\mathrm{P}$. \\ Fixed modifications: \\ Carbamidomethyl (C) \\ Variable modifications: Oxidation (M)
}

\section{Protein sequence coverage: $32 \%$}

Matched peptides shown in bold red.

1 MVKQMTDAQR KAIEWAVKMA ESQIKSSQRR IKRAERSLEL FKGMDELDDK

51 LKRRQIKHQQ NRIESLQNYI DALKSLLD

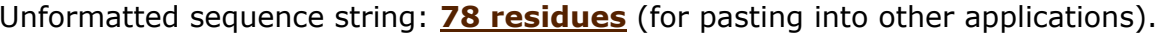

Sort peptides by $\bigcirc$ Residue Number $\bigcirc$ Increasing Mass $\bigcirc$ Decreasing Mass

Show predicted peptides also

\begin{tabular}{|c|c|c|c|c|}
\hline Query & Start - End & Observed & $M r(\exp t)$ & $\operatorname{Mr}(\mathrm{calc})$ \\
\hline$\sigma^{\prime 1153}$ & $12-18$ & 408.7341 & 815.4537 & 815.4541 \\
\hline$\checkmark 301$ & $37-42$ & 368.7160 & 735.4174 & 735.4167 \\
\hline$\checkmark 12 \overline{621}$ & $63-74$ & 703.8797 & 1405.7448 & 1405.74 \\
\hline$\nabla^{\prime 12622}$ & $63-74$ & 703.8815 & 1405.7484 & 1405.74 \\
\hline$\triangle^{\prime 12623}$ & $63-74$ & 703.8817 & 1405.7488 & 1405.7 \\
\hline & 63 & 703.8819 & 1405.7493 & 1405.7 \\
\hline
\end{tabular}

\begin{tabular}{rrr}
\multicolumn{1}{c}{ ppm } & M & Score \\
-0.55 & 0 & 46 \\
0.97 & 0 & 53 \\
-0.30 & 0 & 119 \\
2.27 & 0 & 88 \\
2.53 & 0 & 97 \\
2.91 & 0 & 102
\end{tabular}

Expect Rank

U Peptide

$2.7 e-05 \quad 1$ U K.AIEWAVK.M

$4.7 e-06 \quad 1 \quad$ U R.SLELFK.G

$63-74$

$703.8819-1405.7493 \quad 1405.7453$

$2.910 \quad 102$

$1.2 e-12$

$\begin{array}{llll}1 & \mathrm{U} & \mathrm{R} . \text { IESLONYIDALK.S }\end{array}$

$2.2 e-10 \quad 1$ U R.IESLQNYIDALK.S

$6.1 e-11 \quad 1$ U R.IESLQNYIDALK.S

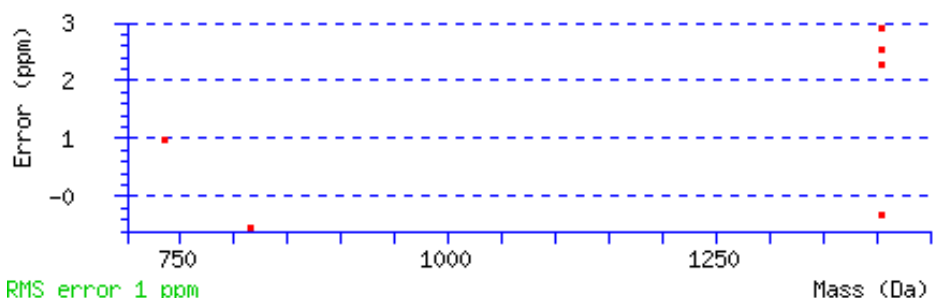




\title{
MASCOT Search Results
}

\section{Protein View: TP84_36}

\author{
Database: $\quad$ TP84 \\ Score: 807 \\ Nominal mass $\left(\mathbf{M}_{\mathbf{r}}\right)$ : 9310 \\ Calculated pI: $\quad 9.91$
}

Sequence similarity is available as an NCBI BLAST search of 6 . against nr.

\section{Search parameters}

MS data file:

Enzyme:

Fixed modifications:

Variable modifications: Oxidation (M)

\section{Protein sequence coverage: $44 \%$}

Matched peptides shown in bold red.

1 MVKQMTDAQR KAIEWAVKMA ESQIKSSQRR IKRAERSLEL FKGMDELDDK 51 LKRRQIKHQQ NRIESLQNYI DALKSLLD

Unformatted sequence string: $\mathbf{7 8}$ residues (for pasting into other applications).

Sort peptides by $\bigcirc$ Residue Number $\bigcirc$ Increasing Mass $\bigcirc$ Decreasing Mass

Show predicted peptides also

\begin{tabular}{|c|c|}
\hline Query & Start - End \\
\hline$\varpi^{\prime} 1990$ & $12-18$ \\
\hline$\varangle^{\prime 556}$ & $37-42$ \\
\hline$\widetilde{\sigma}^{557}$ & $37-42$ \\
\hline$\nearrow^{\prime} \underline{559}$ & $37-42$ \\
\hline$\widetilde{\nearrow}^{560}$ & $37-42$ \\
\hline$\widetilde{\sigma}^{570}$ & $37-42$ \\
\hline$\square^{\prime} \underline{772}$ & $37-42$ \\
\hline$\nabla^{\prime} 13669$ & $43-52$ \\
\hline$\checkmark 13670$ & $43-52$ \\
\hline$\nabla^{13671}$ & $43-52$ \\
\hline$\square^{\prime 13672}$ & $43-52$ \\
\hline$\checkmark 23049$ & $63-74$ \\
\hline$\checkmark \underline{23050}$ & $63-74$ \\
\hline$ð 23051$ & $63-74$ \\
\hline$\checkmark 23052$ & $63-74$ \\
\hline
\end{tabular}

$\begin{array}{lrr}\text { Observed } & \text { Mr(expt) } & \text { Mr(calc) } \\ 408.7365 & 815.4584 & 815.4541 \\ 368.7168 & 735.4190 & 735.4167 \\ 368.7169 & 735.4193 & 735.4167 \\ 368.7170 & 735.4194 & 735.4167 \\ 368.7170 & 735.4195 & 735.4167 \\ 368.7179 & 735.4212 & 735.4167 \\ 368.7181 & 735.4216 & 735.4167 \\ 388.5262 & 1162.5567 & 1162.5540 \\ 388.5264 & 1162.5573 & 1162.5540 \\ 388.5272 & 1162.5599 & 1162.5540 \\ 582.2890 & 1162.5634 & 1162.5540 \\ 703.8814 & 1405.7483 & 1405.7453 \\ 703.8820 & 1405.7494 & 1405.7453 \\ 703.8822 & 1405.7498 & 1405.7453 \\ 703.8827 & 1405.7508 & 1405.7453\end{array}$

$\begin{array}{llr}\text { ppm } & \text { M } & \text { Score } \\ 5.31 & 0 & 53 \\ 3.15 & 0 & 58 \\ 3.58 & 0 & 54 \\ 3.72 & 0 & 58 \\ 3.91 & 0 & 54 \\ 6.11 & 0 & 21 \\ 6.77 & 0 & 58 \\ 2.36 & 1 & 40 \\ 2.85 & 1 & 59 \\ 5.12 & 1 & 57 \\ 8.14 & 1 & 64 \\ 2.16 & 0 & 105 \\ 2.94 & 0 & 102 \\ 3.21 & 0 & 102 \\ 3.98 & 0 & 103\end{array}$

Peptide K.AIEWAVK.M U R.SLELFK.G

U R.SLELFK.G

U R.SLELFK.G

U R. SLELFK.G

U R.SLELFK.G

U R.SLELFK.G

U K.GMDELDDKLK.R

U K. GMDELDDKLK. R

U K.GMDELDDKLK.R

U K. GMDELDDKLK. R

U R. IESLQNYIDALK.S

U R. IESLQNYIDALK.S

$U$ R. IESLQNYIDALK.S

U R. IESLQNYIDALK.S

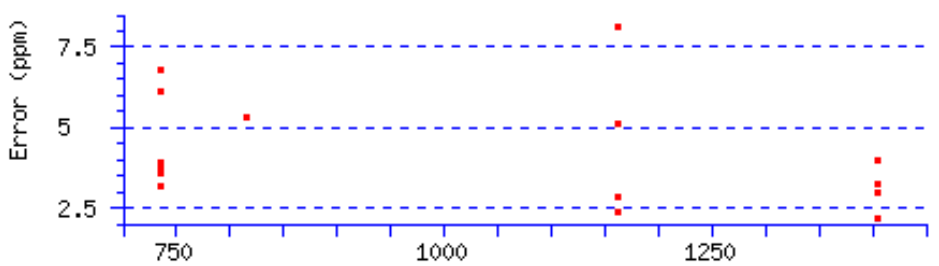




\title{
MASCOT Search Results
}

\section{Protein View: TP84_36}

\author{
Database: TP84 \\ Score: 742 \\ Nominal mass $\left(M_{\mathbf{r}}\right): 9310$ \\ Calculated pI: $\quad 9.91$
}

Sequence similarity is available as an NCBI BLAST search of 6. against nr.

\section{Search parameters}

\section{MS data file: \\ Enzyme: \\ Fixed modifications: \\ Variable modifications: Oxidation (M)}

\section{Protein sequence coverage: $32 \%$}

Matched peptides shown in bold red.

1 MVKQMTDAQR KAIEWAVKMA ESQIKSSQRR IKRAERSLEL FKGMDELDDK 51 LKRRQIKHQQ NRIESLQNYI DALKSLLD

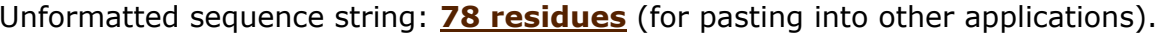

Sort peptides by $\bigcirc$ Residue Number $\bigcirc$ Increasing Mass $\bigcirc$ Decreasing Mass

Show predicted peptides also

\begin{tabular}{|c|c|}
\hline Query & Start - End \\
\hline$\checkmark 1925$ & $4-10$ \\
\hline$\sigma^{\prime 329}$ & $37-42$ \\
\hline 334 & $37-42$ \\
\hline$\sigma^{\prime 15198}$ & $63-74$ \\
\hline$\checkmark 15199$ & $63-74$ \\
\hline$\nabla^{\prime} 15200$ & $63-74$ \\
\hline$\sigma^{\prime 1} 15201$ & $63-74$ \\
\hline$\varpi^{\prime 15202}$ & $63-74$ \\
\hline$\sigma^{\prime 15203}$ & $63-74$ \\
\hline$\sigma^{\prime} 15204$ & $63-74$ \\
\hline
\end{tabular}

$\begin{array}{lrr}\text { Observed } & \text { Mr (expt) } & \text { Mr (calc) } \\ 425.1977 & 848.3809 & 848.3810 \\ 368.7152 & 735.4158 & 735.4167 \\ 368.7157 & 735.4169 & 735.4167 \\ 703.8793 & 1405.7440 & 1405.7453 \\ 703.8795 & 1405.7444 & 1405.7453 \\ 703.8800 & 1405.7455 & 1405.7453 \\ 703.8802 & 1405.7458 & 1405.7453 \\ 703.8804 & 1405.7463 & 1405.7453 \\ 703.8813 & 1405.7481 & 1405.7453 \\ 703.8834 & 1405.7522 & 1405.7453\end{array}$

\begin{tabular}{rrr}
\multicolumn{1}{c}{ ppm } & M & Score \\
-0.21 & 0 & 53 \\
-1.23 & 0 & 50 \\
0.29 & 0 & 58 \\
-0.89 & 0 & 113 \\
-0.57 & 0 & 113 \\
0.21 & 0 & 102 \\
0.42 & 0 & 110 \\
0.72 & 0 & 107 \\
2.03 & 0 & 103 \\
4.97 & 0 & 50
\end{tabular}

Peptide

$\begin{array}{rccl}5 e-06 & 1 & \text { U } & \text { K. QMTDAQR.K } \\ 9.5 e-06 & 1 & \text { U } & \text { R.SLELFK.G }\end{array}$

$1.5 e-06 \quad 1$ U R.SLELFK.G

$5.4 e-12 \quad 1 \quad$ U R.IESLQNYIDALK.S

$5.5 e-12 \quad 1$ U R.IESLQNYIDALK.S

5.7e-11 1 U R.IESLQNYIDALK.S

1e-11 1 U R.IESLQNYIDALK.S

$1.9 e-11 \quad 1 \quad$ U R. IESLQNYIDALK.S

5.5e-11 1 U R. IESLQNYIDALK.S

$9.9 e-06 \quad 1$ U R.IESLQNYIDALK.S

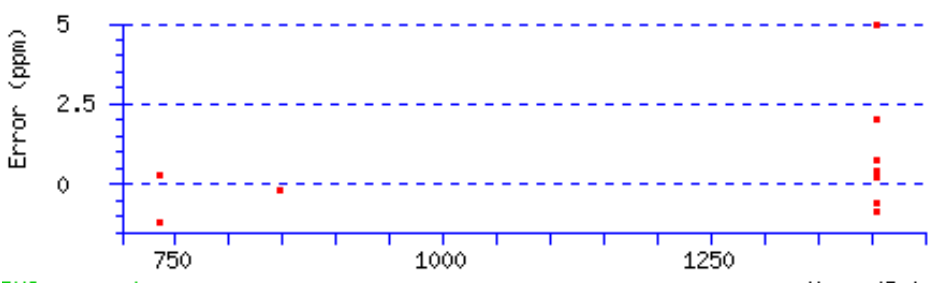

Mascot: http://www.matrixscience.com/ 


\title{
MASCOT Search Results
}

\section{Protein View: TP84_36}

\author{
Database: $\quad$ TP84 \\ Score: $\quad 950$ \\ Nominal mass $\left(\mathbf{M}_{\mathbf{r}}\right)$ : 9310 \\ Calculated pI: $\quad 9.91$
}

Sequence similarity is available as an NCBI BLAST search of 6 . against nr.

\section{Search parameters}

MS data file:

Enzyme:

Fixed modifications:

Variable modifications:

\section{Protein sequence coverage: $23 \%$}

Matched peptides shown in bold red.

1 MVKQMTDAQR KAIEWAVKMA ESQIKSSQRR IKRAERSLEL FKGMDELDDK 51 LKRRQIKHQQ NRIESLQNYI DALKSLLD

Unformatted sequence string: $\mathbf{7 8}$ residues (for pasting into other applications).

Sort peptides by $\bigcirc$ Residue Number $\bigcirc$ Increasing Mass $\bigcirc$ Decreasing Mass

Show predicted peptides also

\begin{tabular}{|c|c|}
\hline Query & Start - End \\
\hline$\sigma^{4} \underline{19}$ & $37-42$ \\
\hline$ð 21415$ & $63-74$ \\
\hline$ð^{\nearrow 21416}$ & $63-74$ \\
\hline$\smile 21417$ & $63-74$ \\
\hline$ð^{21418}$ & $63-74$ \\
\hline$\underbrace{\prime 21419}$ & $63-74$ \\
\hline$\square^{21420}$ & $63-74$ \\
\hline$ð^{\prime 21421}$ & $63-74$ \\
\hline$\checkmark 21422$ & $63-74$ \\
\hline$\checkmark 21423$ & $63-74$ \\
\hline & \\
\hline
\end{tabular}

$\begin{array}{rrr}\text { Observed } & \text { Mr (expt) } & \text { Mr (calc) } \\ 368.7149 & 735.4152 & 735.4167 \\ 703.8789 & 1405.7433 & 1405.7453 \\ 703.8793 & 1405.7441 & 1405.7453 \\ 703.8800 & 1405.7454 & 1405.7453 \\ 703.8807 & 1405.7469 & 1405.7453 \\ 703.8813 & 1405.7479 & 1405.7453 \\ 703.8813 & 1405.7480 & 1405.7453 \\ 703.8817 & 1405.7488 & 1405.7453 \\ 703.8817 & 1405.7489 & 1405.7453 \\ 703.8821 & 1405.7496 & 1405.7453 \\ 703.8828 & 1405.7511 & 1405.7453\end{array}$

\begin{tabular}{rrr}
\multicolumn{1}{c}{ ppm } & M & Score \\
-1.99 & 0 & 58 \\
-1.41 & 0 & 112 \\
-0.80 & 0 & 105 \\
0.11 & 0 & 102 \\
1.16 & 0 & 105 \\
1.92 & 0 & 105 \\
1.93 & 0 & 105 \\
2.54 & 0 & 107 \\
2.58 & 0 & 89 \\
3.10 & 0 & 98 \\
4.19 & 0 & 93
\end{tabular}

Expect Rank U Peptide $\begin{array}{llll}1.5 e-06 & 1 & \mathrm{U} & \text { R.SLELFK.G }\end{array}$ $5.7 e-12$ $3.4 e-11$ 5. $6 e-11$ 3. $3 e-11$ 3. $3 e-11$ 3. $2 e-11$ 1. $9 e-11$ 1. $3 e-09$ 1. $5 e-10$ 4. $6 e-10$
U R. IESLQNYIDALK.S

U R. IESLQNYIDALK.S

U R. IESLQNYIDALK.S

U R. IESLQNYIDALK.S

U R. IESLQNYIDALK. S

$U$ R. IESLQNYIDALK.S

U R. IESLQNYIDALK.S

$U$ R. IESLQNYIDALK.S

U R. IESLQNYIDALK.S

U R. IESLQNYIDALK.S

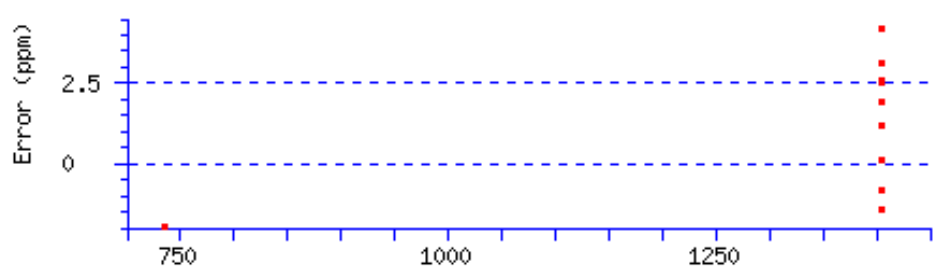

Ris error 2 ppm

Mass (Da)

\section{TP84_36}

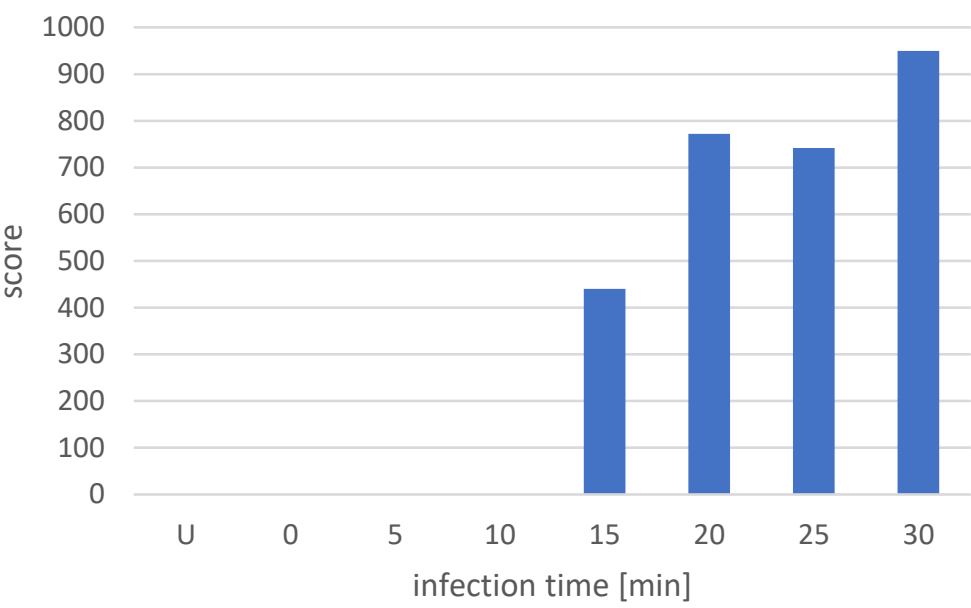




\title{
MASCOT Search Results
}

\section{Protein View: TP84_37}

\author{
Database: TP84 \\ Score: 315 \\ Nominal mass $\left(\mathbf{M}_{\mathbf{r}}\right)$ : 14846 \\ Calculated pI: $\quad 6.41$
}

Sequence similarity is available as an NCBI BLAST search of 8. against nr.

\section{Search parameters}

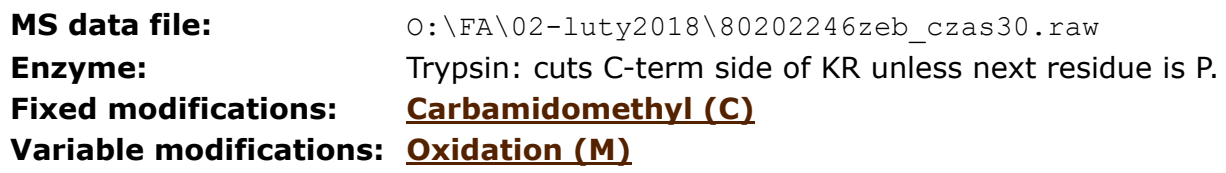

\section{Protein sequence coverage: $15 \%$}

Matched peptides shown in bold red.

1 MQVRKIHKVT YESETMPLEQ AVEEIAQSTG SPVDRVRGLI IQFGKVMTVH

51 HVYHIVDASG IGLVKDAEKF LGQSMVFQLK GGETVILKVM EVTRWMNGDY

101 FIKGYDDEGM WRTIDVDSIK AYFTPGKGMI R

Unformatted sequence string: 131 residues (for pasting into other applications).

Sort peptides by - Residue Number $\bigcirc$ Increasing Mass $\bigcirc$ Decreasing Mass

Show predicted peptides also

$\begin{array}{rr}\text { Query } & \text { Start }- \text { End } \\ \varangle 17191 & 70-80 \\ \square \underline{17192} & 70-80 \\ \square \underline{17193} & 70-80 \\ \square \underline{17194} & 70-80 \\ \square \underline{10565} & 104-112\end{array}$

$\begin{array}{rrr}\text { Observed } & \text { Mr (expt) } & \text { Mr (calc) } \\ 649.3530 & 1296.6915 & 1296.6900 \\ 649.3533 & 1296.6921 & 1296.6900 \\ 649.3534 & 1296.6922 & 1296.6900 \\ 649.3537 & 1296.6928 & 1296.6900 \\ 564.7244 & 1127.4343 & 1127.4342\end{array}$

$\begin{array}{ccc}\text { ppm } & \text { M } & \text { Score } \\ 1.14 & 0 & 77 \\ 1.62 & 0 & 76 \\ 1.68 & 0 & 78 \\ 2.11 & 0 & 80 \\ 0.057 & 0 & 57\end{array}$

Expect Rank 2.1e-08 $2.3 e-08$ $1.7 e-08$ 1. $1 e-08$ $1.9 e-06$

\begin{tabular}{c}
1 \\
$\cdots 1$ \\
$\cdots 1$ \\
-1 \\
1 \\
\hdashline 1 \\
-1
\end{tabular}

U Peptide

U K. FLGQSMVFQLK. G

U K. FLGQSMVFQLK.G

U K.FLGQSMVFQLK.G

U K.FLGQSMVFQLK.G

U K.GYDDEGMWR.T

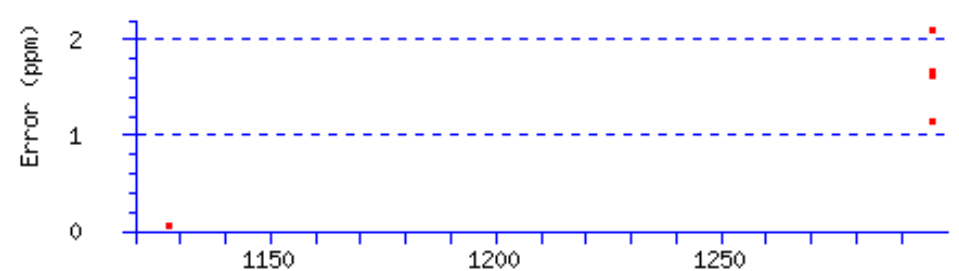

RMS error 1 ppm

Mass (Da)

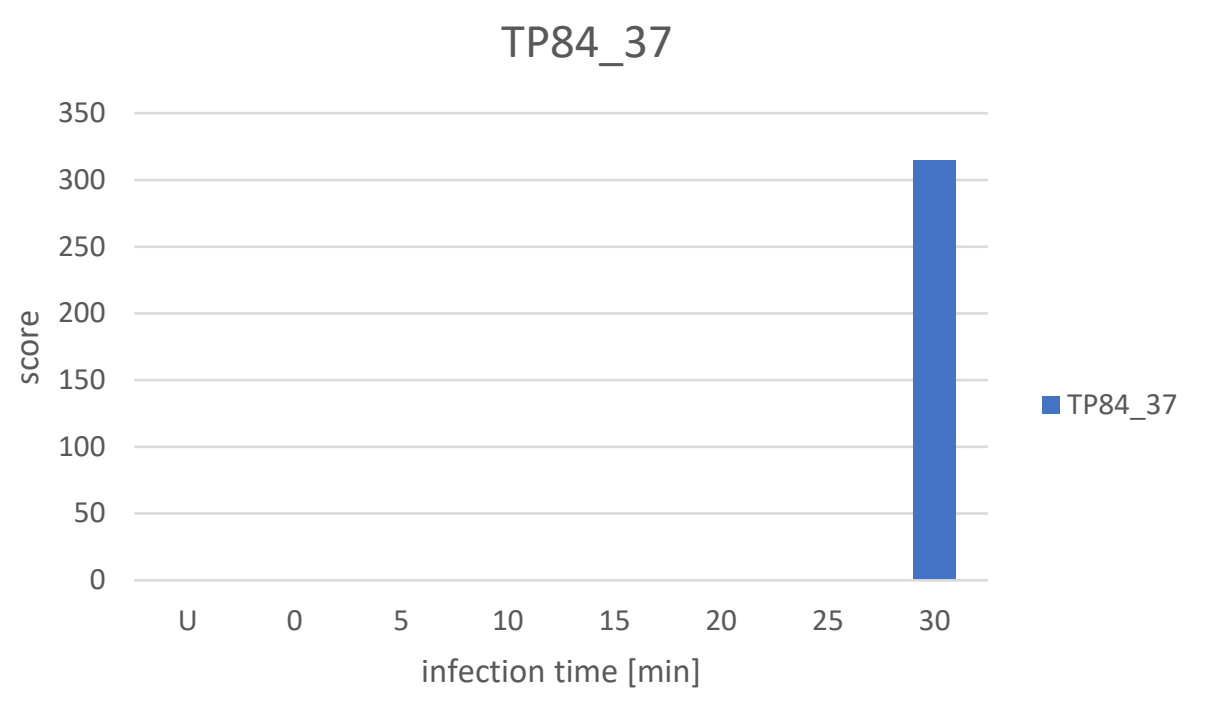




\title{
MASCOT Search Results
}

\section{Protein View: TP84_38}

$\begin{array}{ll}\text { Database: } & \text { TP84 } \\ \text { Score: } & 186 \\ \text { Nominal mass }\left(\mathbf{M}_{\mathbf{r}}\right): & 7380 \\ \text { Calculated pI: } & 6.73\end{array}$

Sequence similarity is available as an NCBI BLAST search of 9. against nr.

\section{Search parameters}

\author{
MS data file: \\ O: \FA $\backslash 02-$ luty2018\80202243zeb_czas15.raw \\ Enzyme: \\ Trypsin: cuts C-term side of KR unless next residue is $\mathrm{P}$. \\ Fixed modifications: \\ Carbamidomethyl (C) \\ Variable modifications: Oxidation (M)
}

\section{Protein sequence coverage: $\mathbf{3 4 \%}$}

Matched peptides shown in bold red.

1 MKPYDPIPMA ITLKPHQTIR KGEVIQETIG GKVVKFKVID IRMVEIRETH

51 ALVGVMAREI GEGDAE

Unformatted sequence string: $\underline{66}$ residues (for pasting into other applications).

Sort peptides by $\bullet$ Residue Number $\bigcirc$ Increasing Mass $\bigcirc$ Decreasing Mass

Show predicted peptides also

$\begin{array}{lc}\text { Query } & \text { Start - End } \\ \varangle \underline{9268} & 21-32 \\ \square \underline{7435} & 48-58 \\ \square \underline{7436} & 48-58\end{array}$

$\begin{array}{rrr}\text { Observed } & \text { Mr (expt) } & \text { Mr (calc) } \\ 629.8534 & 1257.6923 & 1257.6929 \\ 395.2131 & 1182.6174 & 1182.6179 \\ 395.2133 & 1182.6181 & 1182.6179\end{array}$

$\begin{array}{ccc}\text { ppm } & \text { M } & \text { Score } \\ -0.40 & 1 & 122 \\ -0.48 & 0 & 33 \\ 0.15 & 0 & 57\end{array}$

Expect Rank

U Peptide

6. $9 e-131$

U R. KGEVIOETIGGK . V

74436 48 - 58

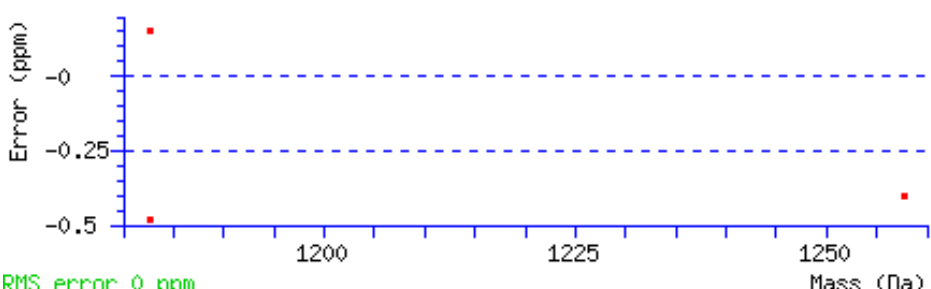




\title{
MASCOT Search Results
}

\section{Protein View: TP84_38}

\author{
Database: TP84 \\ Score: 494 \\ Nominal mass $\left(\mathbf{M}_{\mathbf{r}}\right)$ : 7380 \\ Calculated pI: $\quad 6.73$
}

Sequence similarity is available as an NCBI BLAST search of 9. against nr.

\section{Search parameters}

\author{
MS data file: \\ O: \FA $\backslash 02$-luty2018\80202244zeb czas20.raw \\ Enzyme: \\ Trypsin: cuts C-term side of KR unless next residue is $\mathrm{P}$. \\ Fixed modifications: \\ Carbamidomethyl (C) \\ Variable modifications: Oxidation (M)
}

\section{Protein sequence coverage: $\mathbf{1 8 \%}$}

Matched peptides shown in bold red.

1 MKPYDPIPMA ITLKPHQTIR KGEVIQETIG GKVVKFKVID IRMVEIRETH

51 ALVGVMAREI GEGDAE

Unformatted sequence string: $\underline{66}$ residues (for pasting into other applications).

Sort peptides by $\bigcirc$ Residue Number $\bigcirc$ Increasing Mass $\bigcirc$ Decreasing Mass

Show predicted peptides also

\begin{tabular}{|c|c|}
\hline Query & Start - End \\
\hline$\sigma^{\prime 17637}$ & $21-32$ \\
\hline$\nabla^{\prime 17639}$ & $21-32$ \\
\hline 17640 & $21-32$ \\
\hline$\sigma^{\prime} 17641$ & $21-32$ \\
\hline 17642 & 21 \\
\hline
\end{tabular}

$\begin{array}{rrr}\text { Observed } & \text { Mr (expt) } & \text { Mr (calc) } \\ 629.8556 & 1257.6967 & 1257.6929 \\ 629.8570 & 1257.6995 & 1257.6929 \\ 629.8570 & 1257.6995 & 1257.6929 \\ 629.8572 & 1257.6998 & 1257.6929 \\ 629.8581 & 1257.7017 & 1257.6929\end{array}$

\begin{tabular}{llr} 
Ppm & M & Score \\
3.03 & \multicolumn{1}{|c}{} & 87 \\
5.30 & 1 & 126 \\
5.30 & 1 & 110 \\
5.54 & 1 & 131 \\
7.02 & 1 & 92
\end{tabular}

Expect Rank U Peptide

1.9e-09 1 U R. KGEVIOETIGGK.V

2.6e-13 1 U R.KGEVIQETIGGK.V

1e-11 1 U R.KGEVIQETIGGK.V

$8 e-14 \quad \dddot{1}$ U R.KGEVIQETIGGK.V

$6.6 e-10 \quad 1$ U R.KGEVIQETIGGK.V

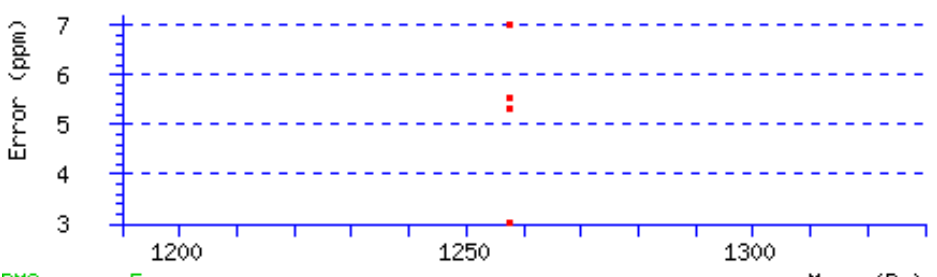

Mascot: http://www.matrixscience.com/ 


\title{
MASCOT Search Results
}

\section{Protein View: TP84_38}

$\begin{array}{ll}\text { Database: } & \text { TP84 } \\ \text { Score: } & 304 \\ \text { Nominal mass }\left(\mathbf{M}_{\mathbf{r}}\right): & 7380 \\ \text { Calculated pI: } & 6.73\end{array}$

Sequence similarity is available as an NCBI BLAST search of 9. against nr.

\section{Search parameters}

\author{
MS data file: \\ O: \FA $\backslash 02$-luty2018\80202245zeb_czas25.raw \\ Enzyme: \\ Trypsin: cuts C-term side of KR unless next residue is $\mathrm{P}$. \\ Fixed modifications: \\ Carbamidomethyl (C) \\ Variable modifications: Oxidation (M)
}

\section{Protein sequence coverage: $\mathbf{3 4 \%}$}

Matched peptides shown in bold red.

1 MKPYDPIPMA ITLKPHQTIR KGEVIQETIG GKVVKFKVID IRMVEIRETH

51 ALVGVMAREI GEGDAE

Unformatted sequence string: $\underline{66}$ residues (for pasting into other applications).

Sort peptides by $\bigcirc$ Residue Number $\bigcirc$ Increasing Mass $\bigcirc$ Decreasing Mass

Show predicted peptides also

\begin{tabular}{|c|c|c|c|c|}
\hline Query & Start - End & Observed & $M r(\exp t)$ & $\operatorname{Mr}(\mathrm{calc})$ \\
\hline$\sigma^{\prime} 11288$ & $21-32$ & 629.8525 & 1257.6904 & 1257 \\
\hline$\checkmark 9100$ & $48-58$ & 592.3179 & 1182.6213 & 1182.617 \\
\hline$\checkmark \overline{9101}$ & $48-58$ & 592.3180 & 1182.6214 & 1182.61 \\
\hline$\triangle^{\prime 9102}$ & $48-58$ & 592.3182 & 1182.6219 & 182 \\
\hline
\end{tabular}

ppm M Score

$-1.931126$

$2.850 \quad 75$

$2.930 \quad 73$

$3.370 \quad 68$
Expect Rank U Peptide

$2.4 e-13 \quad 1 \quad \mathrm{U}$ R. KGEVIOETIGGK.V

$2.8 e-08 \quad 1 \quad$ U R. ETHALVGVMAR.E

$5.2 e-08 \quad 1$ U R.ETHALVGVMAR.E

$1.6 e-07 \quad 1$ U R.ETHALVGVMAR.E

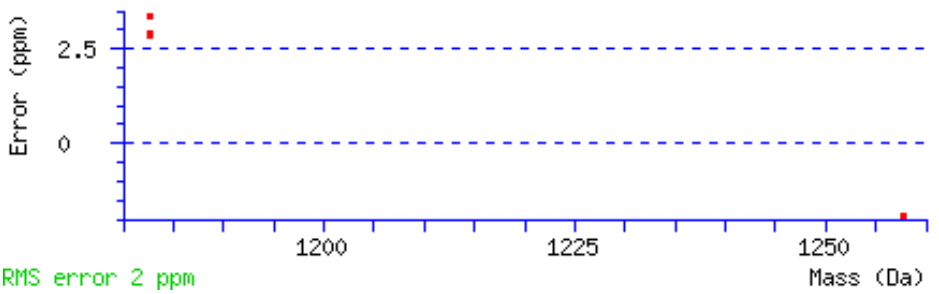




\section{MASCOT Search Results}

\section{Protein View: TP84_38}

$\begin{array}{ll}\text { Database: } & \text { TP84 } \\ \text { Score: } & 420 \\ \text { Nominal mass (Mr): } & 7380 \\ \text { Calculated pI: } & 6.73\end{array}$

Sequence similarity is available as an NCBI BLAST search of 9. against nr.

\section{Search parameters}

MS data file:

Enzyme:

Fixed modifications:

Variable modifications:

\section{Protein sequence coverage: $18 \%$}

Matched peptides shown in bold red.

1 MKPYDPIPMA ITLKPHQTIR KGEVIQETIG GKVVKFKVID IRMVEIRETH

51 ALVGVMAREI GEGDAE

Unformatted sequence string: $\underline{66}$ residues (for pasting into other applications).

Sort peptides by $\bullet$ Residue Number $\bigcirc$ Increasing Mass $\bigcirc$ Decreasing Mass

Show predicted peptides also

$\begin{array}{rr}\text { Query } & \text { Start - End } \\ \nabla^{\prime} 15931 & 21-32 \\ \nabla^{\prime} 15932 & 21-32 \\ \nabla^{\prime} 15936 & 21-32 \\ \sigma^{\prime} 15938 & 21-32\end{array}$

$\begin{array}{rrr}\text { Observed } & \text { Mr (expt) } & \text { Mr (calc) } \\ 629.8529 & 1257.6913 & 1257.6929 \\ 629.8531 & 1257.6915 & 1257.6929 \\ 629.8536 & 1257.6926 & 1257.6929 \\ 629.8548 & 1257.6950 & 1257.6929\end{array}$

ppm M Score

$-1.201108$

$-1.041121$

$-0.211108$

1.711122
Expect Rank U Peptide

$\begin{array}{llll}1.5 e-11 & 1 & \mathrm{U} & \mathrm{R} . \text { KGEVIQETIGGK.V }\end{array}$

$7.6 e-13 \quad \because \quad$ U R.KGEVIQETIGGK.V

$1.6 e-11 \quad 1$ U R.KGEVIQETIGGK.V

$7.1 \mathrm{e}-13$ i 13 U R.KGEVIQETIGGK.V

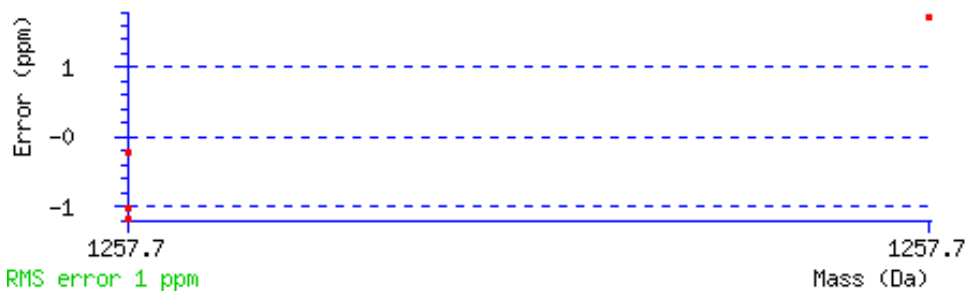

TP84_38

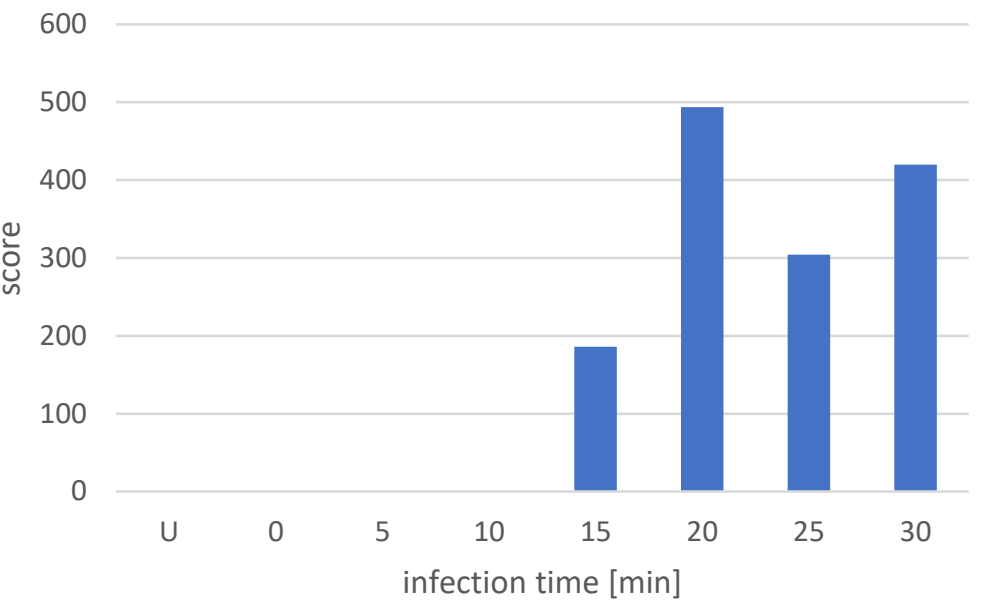




\title{
MASCOT Search Results
}

\section{Protein View: TP84 53}

\author{
Database: TP84 \\ Score: 333 \\ Nominal mass $\left(\mathbf{M}_{\mathbf{r}}\right): 38747$ \\ Calculated pI: $\quad 5.33$
}

Sequence similarity is available as an NCBI BLAST search of 25. against $\mathbf{n r}$.

\section{Search parameters}

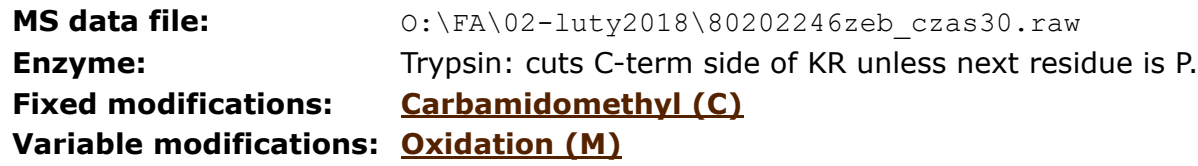

\section{Protein sequence coverage: $10 \%$}

Matched peptides shown in bold red.

\footnotetext{
1 MRKWAVLTDD LKFCEITFDG EWYRVRITDE FGQELMTNES ISLQGAKKQV

51 KRFIGTGQTL KWQEVETDKI ETPSKLHLPF GYLSVSQVRK YLTCPRAYEF

101 KYVNKLNEPI GSTLVMGRAF HKGMQMASIK KVVDGEILST DDVLDVYSDA

151 FDQERENNDV DWAEDDPAKV KDDGAKLMQK YYEEMGINAI PMVDDRGLPL

201 VEREHAFEIV PGLKAKAVID IIEQDGSIRD YKTSKRSPSQ TIIDETIQMP

251 VYALAYRDIT GQVEKTVGLD YAVNLKKEKK IMRLETDGPV DDGRIERVKQ

301 TFVGVAKAIS AGIFYPNEES NACGYCSFKD ICKKSKTF
}

Unformatted sequence string: $\mathbf{3 3 8}$ residues (for pasting into other applications).

Sort peptides by $\odot$ Residue Number $\bigcirc$ Increasing Mass $\bigcirc$ Decreasing Mass

Show predicted peptides also

\begin{tabular}{|c|c|c|c|c|}
\hline Query & Start - End & Observed & $M r(\exp t)$ & $\operatorname{Mr}(\mathrm{calc})$ \\
\hline$\underbrace{\prime 20795}$ & $106-118$ & 693.8754 & 1385.7362 & 1385.7337 \\
\hline$\triangle 20796$ & $106-118$ & 693.8775 & 1385.7404 & 1385.7337 \\
\hline$\checkmark 14975$ & $204-214$ & 620.3402 & 1238.6659 & 1238.6659 \\
\hline$\sigma^{\prime} 12441$ & $284-294$ & 587.2735 & 1172.5325 & 1172 \\
\hline$\sigma^{\prime} 12442$ & $284-294$ & 587.2737 & 1172.5329 & 1172.5310 \\
\hline
\end{tabular}

\begin{tabular}{rrrrc}
\multicolumn{1}{c}{ Ppm } & M & Score & Expect & Rank \\
1.80 & 0 & 106 & $2.4 e-11$ & 1 \\
4.87 & 0 & 88 & $1.8 e-09$ & 1 \\
-0.033 & 0 & 61 & $8.1 e-07$ & 1 \\
1.32 & 0 & 64 & $4.1 e-07$ & 1 \\
1.64 & 0 & 67 & $2.2 e-07$ & $\frac{1}{1}$
\end{tabular}

U Peptide

U K. LNEPIGSTLVMGR. A

U K. LNEPIGSTLVMGR. A

U R. EHAFEIVPGLK. A

-12442 $284-294$

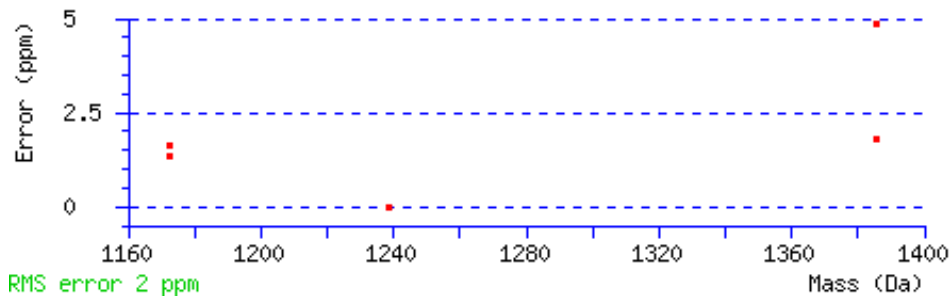

TP84_53

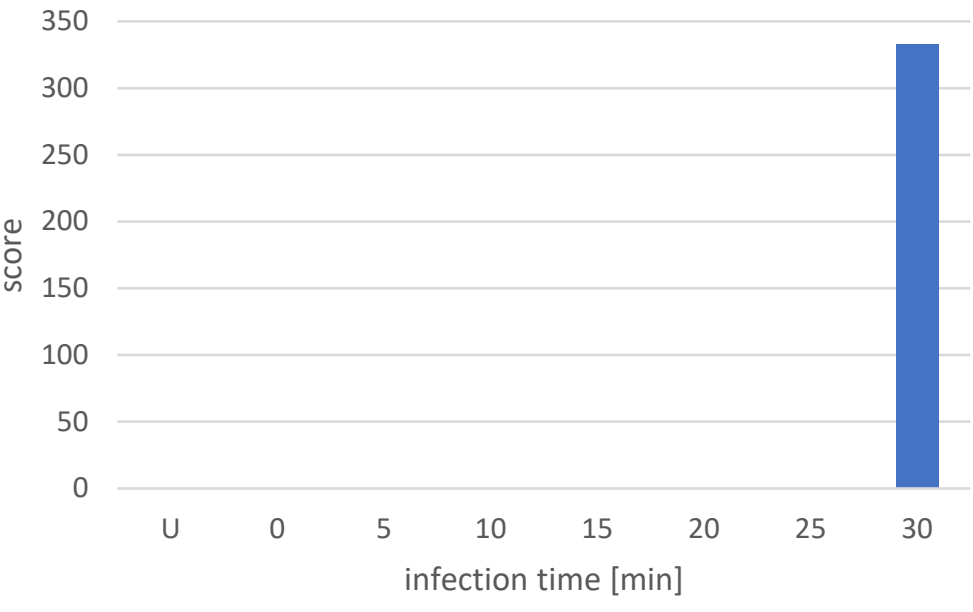




\section{MASCOT Search Results}

\section{Protein View: TP84_54}

$\begin{array}{ll}\text { Database: } & \text { TP84 } \\ \text { Score: } & 542 \\ \text { Nominal mass (Mr): } & 38765 \\ \text { Calculated pI: } & 5.78\end{array}$

Sequence similarity is available as an NCBI BLAST search of 26. against nr.

\section{Search parameters}

MS data file:

Enzyme:

Fixed modifications:

Variable modifications:

O: \FA $\backslash 02-1$ uty2018\80202246zeb czas30.raw

Trypsin: cuts $\mathrm{C}$-term side of $\mathrm{KR}$ unless next residue is $\mathrm{P}$.

Carbamidomethyl (C)

Oxidation (M)

\section{Protein sequence coverage: 14\%}

Matched peptides shown in bold red.

1 MGFDMQYFSE KEAQQYQDAV EKLTEQNTIQ VVSLNDIKQK LLEEVKEFEF

51 SPLEYVPGPD ETQKTIFLKI ANIMGELDRI PKSGYNKQFD YYYVTESDVV

101 GAVRPLMAKY KLVMMPSLKK YKTETIQGKY NKMIMGTVEI EWIVRDAESY

151 EQIKFTMLGK GLDNLEKDIY KAITGNKKYA LITLFMIDSG DDPERNDTPT

201 GEEGPQNAQN GPENTKKTQG NSKTPGQPKN GQEVPKNASQ GQPQQPPQQP

251 PVKQPTKGDL LTRWVVLAGP DQKKQEVRKA FDEWYEKKKA EGWDHMAMMQ

301 ALTKKLHEKN QKEKQAQQQQ ADGQSGQAQQ QQTDDKSKE

Unformatted sequence string: $\mathbf{3 3 9}$ residues (for pasting into other applications).

Sort peptides by $\odot$ Residue Number $\bigcirc$ Increasing Mass $\bigcirc$ Decreasing Mass

Show predicted peptides also

\begin{tabular}{|c|c|}
\hline Query & Start - End \\
\hline$\varnothing^{\prime 17755}$ & $12-22$ \\
\hline '38893 & $47-64$ \\
\hline 38894 & $47-64$ \\
\hline “38895 & $47-64$ \\
\hline$\sigma^{\prime 38896}$ & $47-64$ \\
\hline$\nearrow^{9} \underline{9112}$ & $146-154$ \\
\hline$\checkmark \underline{9113}$ & $146-154$ \\
\hline$\checkmark \underline{9114}$ & $146-154$ \\
\hline$\triangle 0 \underline{9115}$ & $146-154$ \\
\hline$\nearrow^{\prime} 10116$ & $264-273$ \\
\hline
\end{tabular}

$\begin{array}{rrr}\text { Observed } & \text { Mr (expt) } & \text { Mr (calc) } \\ 654.8074 & 1307.6002 & 1307.5993 \\ 1056.4870 & 2110.9594 & 2110.9735 \\ 1056.4876 & 2110.9606 & 2110.9735 \\ 1056.4885 & 2110.9624 & 2110.9735 \\ 1056.4921 & 2110.9696 & 2110.9735 \\ 541.7542 & 1081.4938 & 1081.4927 \\ 541.7544 & 1081.4942 & 1081.4927 \\ 541.7549 & 1081.4952 & 1081.4927 \\ 541.7550 & 1081.4954 & 1081.4927 \\ 556.8080 & 1111.6015 & 1111.6026\end{array}$

$\begin{array}{ccc}\text { ppm } & \text { M } & \text { Score } \\ 0.64 & 0 & 72 \\ -6.66 & 0 & 69 \\ -6.09 & 0 & 75 \\ -5.24 & 0 & 71 \\ -1.83 & 0 & 81 \\ 0.95 & 0 & 58 \\ 1.40 & 0 & 60 \\ 2.30 & 0 & 60 \\ 2.45 & 0 & 53 \\ -0.98 & 0 & 60\end{array}$

$\begin{array}{rc}\text { Expect } & \text { Rank } \\ 6.3 e-08 & 1 \\ 1.3 e-07 & 1 \\ 2.9 e-08 & 1 \\ 8 e-08 & 1 \\ 8.1 e-09 & -1 \\ 1.7 e-06 & -1 \\ 9.8 e-07 & 1 \\ 1 e-06 & -1 \\ 4 . \\ 8 e-06 & 1 \\ 1 e-06 & 1\end{array}$

U Peptide

U K.EAQQYQDAVEK. I

U K.EFEFSPLEYVPGPDETQK. T

U K.EFEFSPLEYVPGPDETQK.T

U K.EFEFSPLEYVPGPDETQK . T

U K.EFEFSPLEYVPGPDETQK.T

U R.DAESYEQIK. F

U R.DAESYEOIK. F

U R.DAESYEQIK. F

U R. DAESYEOIK. F

U R.WVVLAGPDQK.K

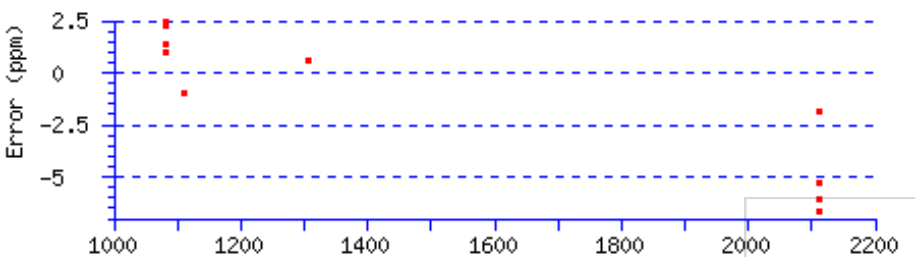

RMS error $3 \mathrm{pmm}$ Mass (Da)

TP84_54

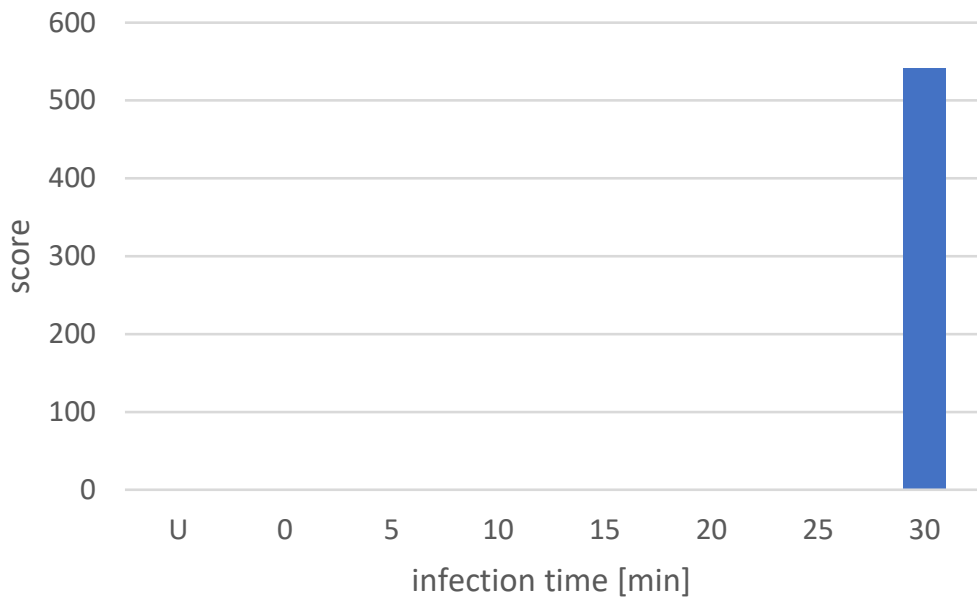




\title{
MASCOT Search Results
}

\section{Protein View: TP84_56}

\author{
Database: $\quad$ TP84 \\ Score: 271 \\ Nominal mass $\left(\mathbf{M}_{\mathbf{r}}\right)$ : 7416 \\ Calculated pI: $\quad 9.25$
}

Sequence similarity is available as an NCBI BLAST search of 28. against nr.

\section{Search parameters}

MS data file:

O: \FA 02 -luty2018\80202244zeb_czas20.raw

Enzyme:

Trypsin: cuts C-term side of KR unless next residue is $\mathrm{P}$.

Fixed modifications: Carbamidomethyl (C)

Variable modifications: Oxidation (M)

\section{Protein sequence coverage: $\mathbf{5 0 \%}$}

Matched peptides shown in bold red.

1 MEKKQKFNPI LSFQTDESVC EFIQKVMIKR MEFNRSNIVR EIFMIGLEEF

51 KKRHPEIDKK

Unformatted sequence string: $\underline{60}$ residues (for pasting into other applications).

Sort peptides by $\odot$ Residue Number $\bigcirc$ Increasing Mass $\bigcirc$ Decreasing Mass

Show predicted peptides also

\begin{tabular}{|c|c|c|c|c|c|c|c|c|c|c|c|}
\hline Query & Start - End & Observed & $M r(\exp t)$ & $\operatorname{Mr}(\operatorname{calc})$ & ppm & M & Score & Expect & Rank & $\mathrm{U}$ & Peptide \\
\hline$\smile^{\prime 4} \underline{1727}$ & $7-25$ & 1151.5592 & 2301.1038 & 2301.0987 & 2.22 & $\ddot{0}$ & 73 & $5.5 e-08$ & 1 & $\ddot{\mathrm{U}}$ & K. FNPILSFQTDESVCEFIQK.V \\
\hline$\nearrow^{\prime 2} \underline{1210}$ & $41-51$ & 678.3491 & 1354.6837 & 1354.6843 & -0.43 & 0 & 53 & $4.7 e-06$ & 1 & $\mathrm{U}$ & R.EIFMIGLEEFK.K \\
\hline$\nearrow^{\prime 2} \underline{1211}$ & $41-51$ & 678.3494 & 1354.6843 & 1354.6843 & 0.013 & 0 & 69 & $1.3 e-07$ & 1 & $\mathrm{U}$ & R.EIFMIGLEEFK. K \\
\hline$\checkmark 21212$ & $41-51$ & 678.3499 & 1354.6852 & 1354.6843 & 0.69 & 0 & 62 & $7.1 e-07$ & 1 & $\mathrm{U}$ & R.EIFMIGLEEFK.K \\
\hline$\overline{21213}$ & $41-51$ & 678.3502 & 1354.6858 & 1354.6843 & 1.18 & 0 & 67 & $1.9 e-07$ & 1 & $\mathrm{U}$ & R.EIFMIGLEEFK.K \\
\hline
\end{tabular}
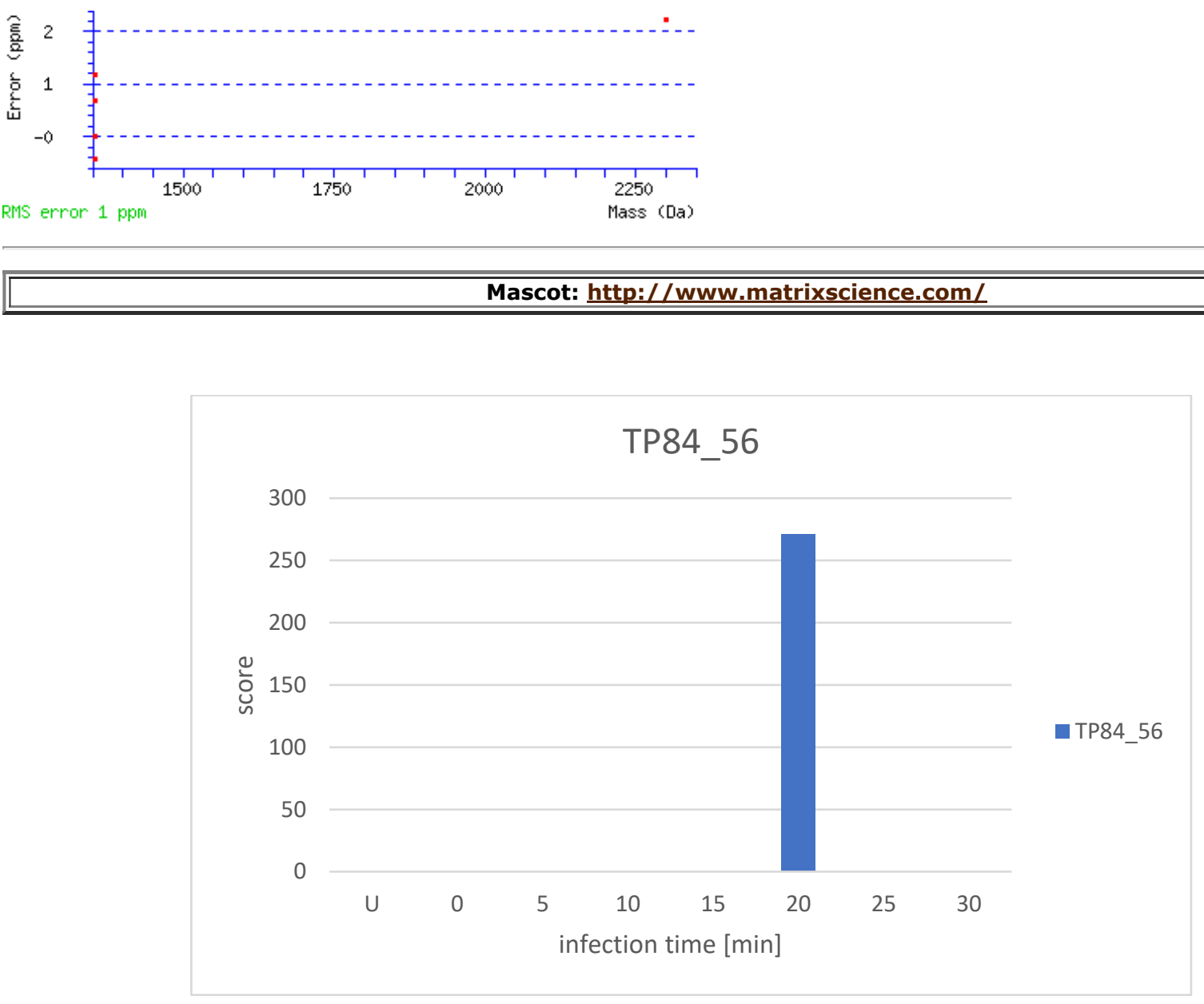


\section{MASCOT Search Results}

\section{Protein View: TP84_57}

$\begin{array}{ll}\text { Database: } & \text { TP84 } \\ \text { Score: } & 338 \\ \text { Nominal mass (Mr): } & 30368 \\ \text { Calculated pI: } & 8.57\end{array}$

Sequence similarity is available as an NCBI BLAST search of 29. against nr.

\section{Search parameters}

MS data file:

Enzyme:

$0: \backslash F A \backslash 02-1$ uty2018\80202246zeb_czas30.raw

Fixed modifications:

Trypsin: cuts $\mathrm{C}$-term side of $\mathrm{KR}$ unless next residue is $\mathrm{P}$.

Variable modifications: Oxidation (M)

\section{Protein sequence coverage: $10 \%$}

Matched peptides shown in bold red.

1 MSDQEKQEYL MSDDMWVYGY SVIDNGVIYS KEITDSEFRT YCVIRSLVNE

51 RKAVAWPSYE TIAELSGHSK RTAMRNVARL IELDLIEKRP RSGTSNEFVV

101 KKLQNSKVLK NKQDILDYIE KCRDDEPKKA TDPGEKVDKP EKADPIPYKE

151 IIDYLNEKAG TKYSHTGSAN QKLIKARWNE MAKINKDRDW IVAQFKHVID

201 VKTAQWKGTE WEKYLRPSTL FGNKFDQYRN ESPNHKPVGG QNRPKEDIRT

251 RLAGFLADED

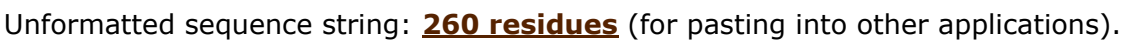

Sort peptides by $\bullet$ Residue Number $\bigcirc$ Increasing Mass $\bigcirc$ Decreasing Mass

Show predicted peptides also

$\begin{array}{rc}\text { Query } & \text { Start - End } \\ \nabla^{36376} & 53-70 \\ \nabla^{36377} & 53-70 \\ \nabla^{36378} & 53-70 \\ \square \underline{9242} & 80-88\end{array}$

Observed Mr (expt) Mr (calc)

$649.3261 \quad 1944.9563 \quad 1944.9581$

$649.3286 \quad 1944.9641 \quad 1944.9581$

$649.3287 \quad 1944.9644 \quad 1944.9581$

$543.3270 \quad 1084.6394 \quad 1084.6379$ ppm M Score

$-0.920104$

$3.060 \quad 84$

$3.210 \quad 99$

$1.370 \quad 89$
Expect Rank U Peptide

3. $9 e-11 \quad 1$ U K.AVAWPSYETIAELSGHSK.R

4.2e-09 1 U K.AVAWPSYETIAELSGHSK.R

$1.1 e-10$ i U K.AVAWPSYETIAELSGHSK.R 1. $2 e-09 \quad 1$

U R. LIELDLIEK. R

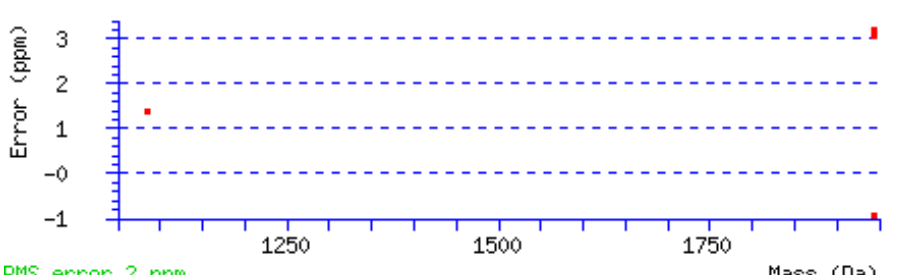
RMS error 2 ppm
Mass (Da)

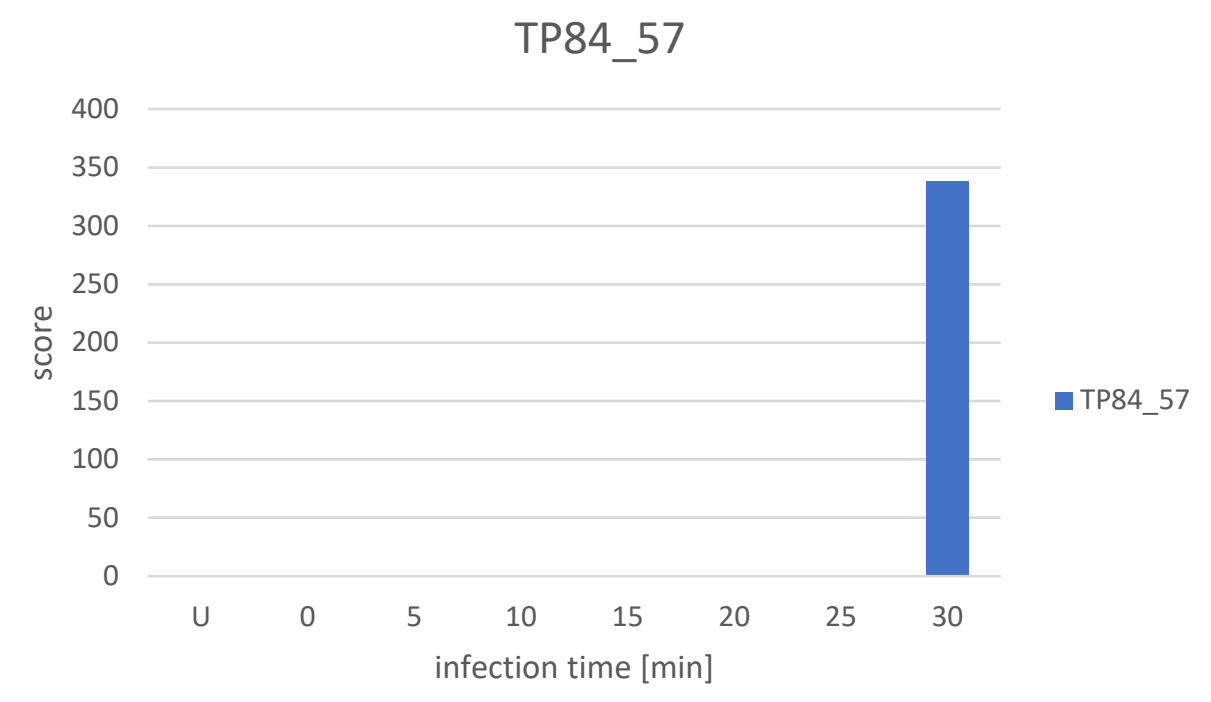




\section{MASCOT Search Results}

\section{Protein View: TP84_63}

$\begin{array}{ll}\text { Database: } & \text { TP84 } \\ \text { Score: } & 357 \\ \text { Nominal mass (Mr): } & 17391 \\ \text { Calculated pI: } & 5.16\end{array}$

Sequence similarity is available as an NCBI BLAST search of 35 . against nr.

\section{Search parameters}

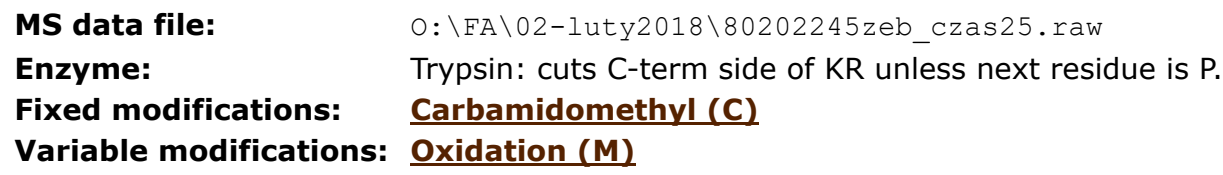

\section{Protein sequence coverage: $18 \%$}

Matched peptides shown in bold red.

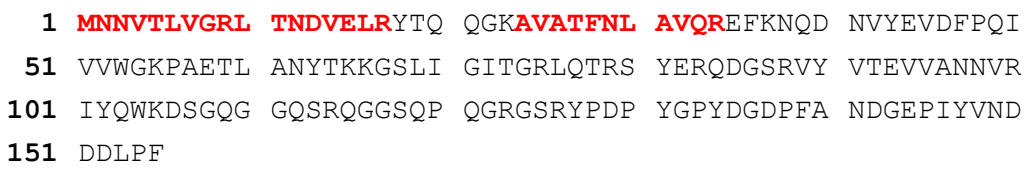

Unformatted sequence string: 155 residues (for pasting into other applications).

Sort peptides by $\odot$ Residue Number $\bigcirc$ Increasing Mass $\bigcirc$ Decreasing Mass

Show predicted peptides also

\begin{tabular}{|c|c|}
\hline Query & Start - End \\
\hline$\nearrow^{5004}$ & $1-9$ \\
\hline$\checkmark \underline{5005}$ & $1-9$ \\
\hline$\checkmark 3934$ & $10-17$ \\
\hline$\checkmark \underline{3936}$ & $10-17$ \\
\hline$\nearrow^{3} \underline{3938}$ & $10-17$ \\
\hline$\checkmark 3939$ & $10-17$ \\
\hline$\checkmark 9300$ & $24-34$ \\
\hline$\triangle 9301$ & $24-34$ \\
\hline
\end{tabular}

$\begin{array}{rrr}\text { Observed } & \text { Mr (expt) } & \text { Mr (calc) } \\ 502.2718 & 1002.5290 & 1002.5280 \\ 502.2718 & 1002.5291 & 1002.5280 \\ 480.2616 & 958.5087 & 958.5083 \\ 480.2618 & 958.5091 & 958.5083 \\ 480.2619 & 958.5092 & 958.5083 \\ 480.2621 & 958.5097 & 958.5083 \\ 595.3336 & 1188.6527 & 1188.6615 \\ 595.3390 & 1188.6634 & 1188.6615\end{array}$

$\begin{array}{ccc}\text { ppm } & \text { M } & \text { Score } \\ 0.92 & 0 & 59 \\ 1.08 & 0 & 60 \\ 0.33 & 0 & 53 \\ 0.81 & 0 & 51 \\ 0.94 & 0 & 52 \\ 1.38 & 0 & 52 \\ -7.38 & 0 & 50 \\ 1.62 & 0 & 71\end{array}$

$\begin{array}{rc}\text { Expect } & \text { Rank } \\ 1.1 e-06 & 1 \\ 1 e-06 & 1 \\ 5.4 e-06 & 1 \\ 8.4 e-06 & 1 \\ 6.3 e-06 & 1 \\ 6.1 e-06 & 1 \\ 9.6 e-06 & 1 \\ 8.8 e-08 & 1\end{array}$

U Peptide

Ü - .MNNVTLVGR. L

$\mathrm{U} \quad$ - . MNNVTLVGR.L

U R.LTNDVELR.Y

U R. LTNDVELR.Y

U R. LTNDVELR.Y

U R.LTNDVELR.Y

U K.AVATFNLAVQR.E $595.3390 \quad 1188.6634 \quad 1188.6615$

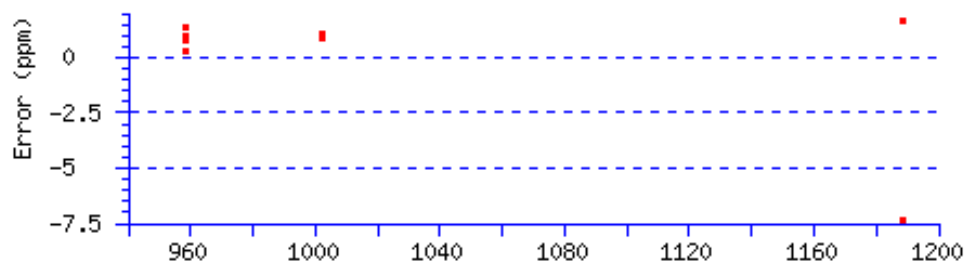

RMS error 2 ppm

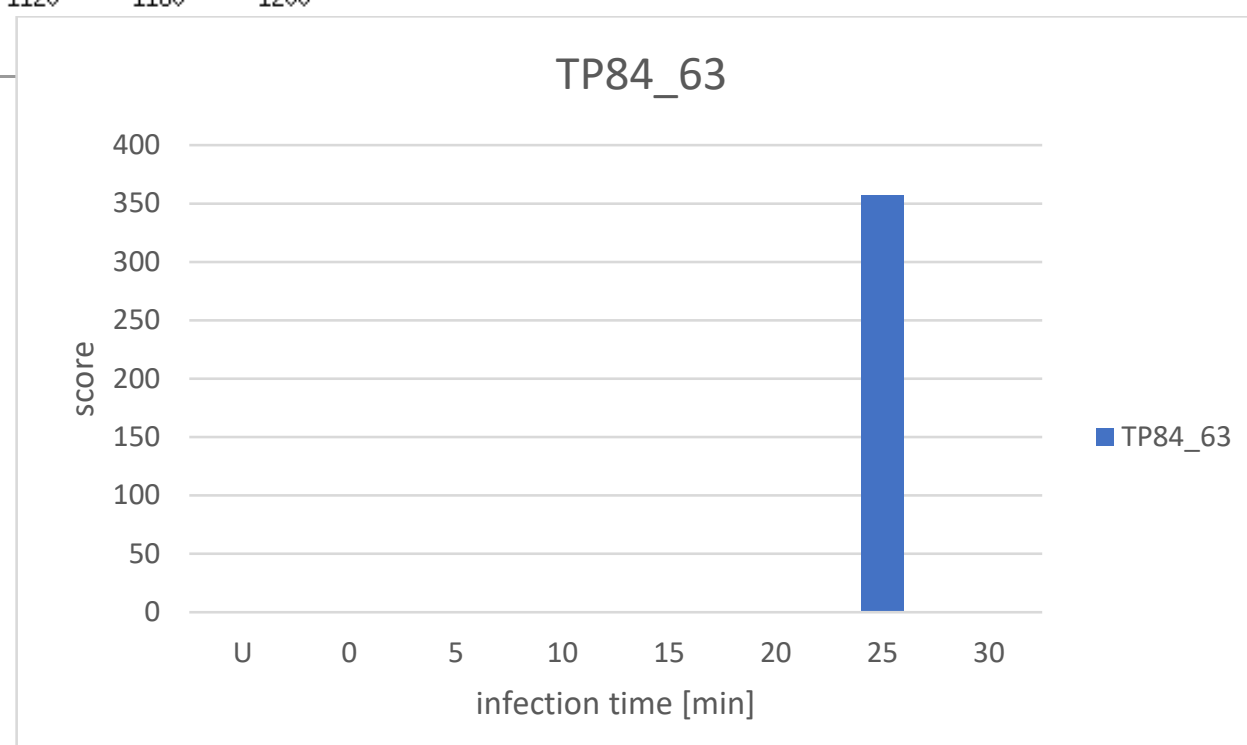




\title{
MASCOT Search Results
}

\section{Protein View: TP84_64}

\author{
Database: TP84 \\ Score: 232 \\ Nominal mass $\left(\mathbf{M}_{\mathbf{r}}\right)$ : 9330 \\ Calculated pI: $\quad 6.89$
}

Sequence similarity is available as an NCBI BLAST search of 36 . against nr.

\section{Search parameters}

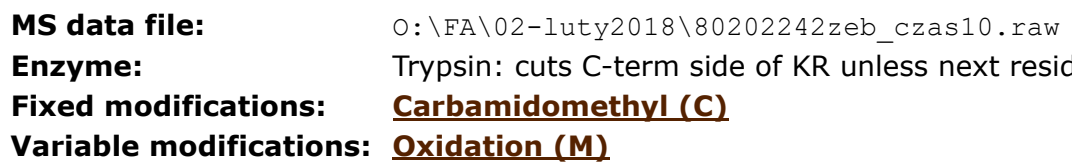

\section{Protein sequence coverage: $\mathbf{2 7 \%}$}

Matched peptides shown in bold red.

1 MVKYFVGSDV HFIAFDQNLF KHDKCFHIKR SDQIYSGITI YKVAGSQIAD

51 YLHDRIMNMY IKGDINLEVM PESLLYKTI

Unformatted sequence string: $\underline{79}$ residues (for pasting into other applications).

Sort peptides by $\bullet$ Residue Number $\bigcirc$ Increasing Mass $\bigcirc$ Decreasing Mass

Show predicted peptides also

\begin{tabular}{|c|c|c|c|c|}
\hline Query & Start - End & Observed & $M r$ (expt) & $\operatorname{Mr}(\mathrm{calc})$ \\
\hline$\checkmark 2335$ & $56-62$ & 456.7388 & 911.4631 & 911.4608 \\
\hline 19412 & $63-77$ & 860.9433 & 1719.8720 & 1719.8753 \\
\hline 19413 & $63-77$ & 860.9444 & 1719.8743 & 1719.8753 \\
\hline 1 & $63-77$ & 860.9450 & 1719.8754 & 1719 \\
\hline
\end{tabular}

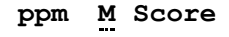

2.460

$-1.900$

$-0.590$

$860.9450 \quad 1719.8754 \quad 1719.8753$

31
68
83
89

$\begin{array}{rc}\text { Expect } & \text { Rank } \\ 0.00089 & 1 \\ 1.4 e-07 & 1 \\ 5.6 e-09 & \frac{1}{1} \\ 1.2 e-09 & 1\end{array}$

U Peptide

U R. IMNMYIK.G

U K.GDINLEVMPESLLYK.T

U K.GDINLEVMPESLLYK.T

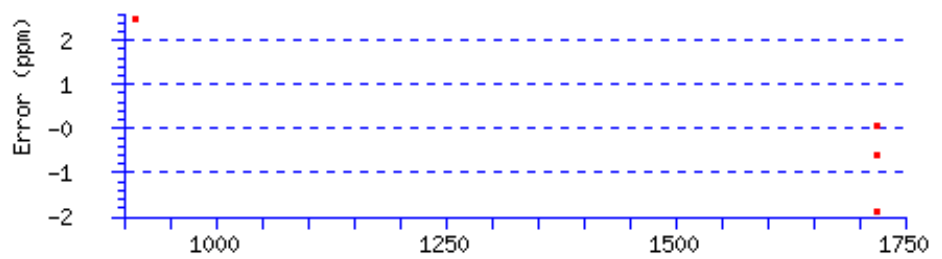

RIS error 1 ppri Mass (Da) 


\section{MASCOT Search Results}

\section{Protein View: TP84_64}
Database:
TP84
Score:
200
Nominal mass $\left(\mathbf{M}_{\mathbf{r}}\right): 9330$
Calculated pI:
6.89

Sequence similarity is available as an NCBI BLAST search of 36. against nr.

\section{Search parameters}

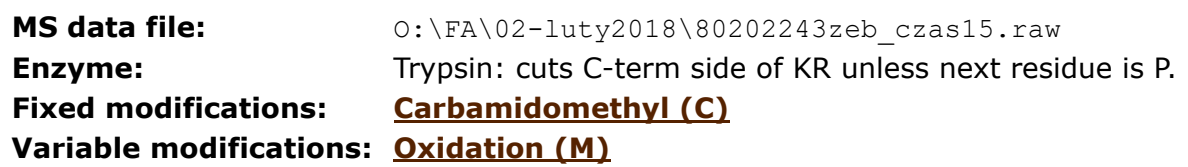

\section{Protein sequence coverage: $\mathbf{5 0 \%}$}

Matched peptides shown in bold red.

1 MVKYFVGSDV HFIAFDQNLF KHDKCFHIKR SDQIYSGITI YKVAGSQIAD

51 YLHDRIMNMY IKGDINLEVM PESLLYKTI

Unformatted sequence string: $\underline{79}$ residues (for pasting into other applications).

Sort peptides by $\odot$ Residue Number $\bigcirc$ Increasing Mass $\bigcirc$ Decreasing Mass

Show predicted peptides also

$\begin{array}{rr}\text { Query } & \text { Start }- \text { End } \\ \nabla^{\prime} 12268 & 31-42 \\ \nabla^{\prime} 12269 & 31-42 \\ \nabla^{\prime} 13458 & 43-55 \\ \nabla^{\prime} 13459 & 43-55 \\ \nabla^{\prime} 19316 & 63-77\end{array}$

$\begin{array}{ccc}\text { ppm } & \text { M } & \text { Score } \\ -3.43 & 0 & 22 \\ -1.00 & 0 & 14 \\ -1.48 & 0 & 70 \\ -0.069 & 0 & 51 \\ -1.78 & 0 & 96\end{array}$

Expect Rank U Peptide

$0.0063 \quad 1 \quad \dddot{U} \quad$ R.SDQIYSGITIYK.V

$0.043 \quad 1 \quad$ U R.SDOIYSGITIYK.V

$1 e-07 \quad 1$ U K.VAGSQIADYLHDR.I

8.1e-06 1 U K.VAGSOIADYLHDR. I

$2.7 e-10 \quad \frac{U}{1}$ K.GDINLEVMPESLLYK.T

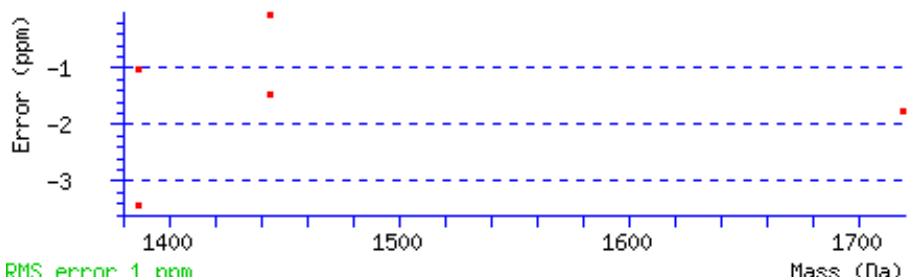




\section{MASCOT Search Results}

\section{Protein View: TP84_64}

$\begin{array}{ll}\text { Database: } & \text { TP84 } \\ \text { Score: } & 522 \\ \text { Nominal mass }\left(\mathbf{M}_{\mathbf{r}}\right): & 9330 \\ \text { Calculated pI: } & 6.89\end{array}$

Sequence similarity is available as an NCBI BLAST search of 36 . against nr.

\section{Search parameters}

MS data file:

Enzyme:

D: \FA $\backslash 02-1$ uty2018\80202244zeb_czas20.raw

\section{Fixed modifications:}

Trypsin: cuts $\mathrm{C}$-term side of KR unless next residue is $\mathrm{P}$.

Variable modifications: Oxidation (M)

\section{Protein sequence coverage: $\mathbf{5 8 \%}$}

Matched peptides shown in bold red.

1 MVKYFVGSDV HFIAFDQNLF KHDKCFHIKR SDQIYSGITI YKVAGSQIAD

51 YLHDRIMNMY IKGDINLEVM PESLLYKTI

Unformatted sequence string: $\underline{79}$ residues (for pasting into other applications).

Sort peptides by $\odot$ Residue Number $\bigcirc$ Increasing Mass $\bigcirc$ Decreasing Mass

Show predicted peptides also

\begin{tabular}{|c|c|}
\hline Query & Start - End \\
\hline$\underbrace{\prime 3} 39718$ & $4-21$ \\
\hline$\underbrace{24184}$ & $43-55$ \\
\hline$\triangle 24185$ & $43-55$ \\
\hline$\varpi^{\prime 2} \underline{4186}$ & $43-55$ \\
\hline$\square^{\prime 24187}$ & $43-55$ \\
\hline$\square^{24188}$ & $43-55$ \\
\hline '32080 & $63-77$ \\
\hline
\end{tabular}

$\begin{array}{rrr}\text { Observed } & \text { Mr (expt) } & M r \text { (calc) } \\ 716.3572 & 2146.0497 & 2146.0524 \\ 482.2466 & 1443.7179 & 1443.7106 \\ 482.2466 & 1443.7181 & 1443.7106 \\ 722.8665 & 1443.7184 & 1443.7106 \\ 482.2468 & 1443.7186 & 1443.7106 \\ 722.8670 & 1443.7194 & 1443.7106 \\ 860.9463 & 1719.8780 & 1719.8753\end{array}$

\begin{tabular}{rrr}
\multicolumn{1}{c}{ ppm } & M & Score \\
-1.25 & 0 & 59 \\
5.04 & 0 & 69 \\
5.15 & 0 & 73 \\
5.39 & 0 & 122 \\
5.54 & 0 & 79 \\
6.09 & 0 & 116 \\
1.57 & 0 & 82
\end{tabular}

Expect Rank

Peptide

$.4 e-06$

VHFIAFDONLFK .

$63-77$

$860.9463 \quad 1719.8780 \quad 1719.8753$

$1.570 \quad 82$

$1.4 e-07$

U K.VAGSQIADYLHDR. I

4e-08 1 U K.VAGSQIADYLHDR. I

$5.6 e-13 \quad 1$ U K.VAGSQIADYLHDR. I

$1.3 e-08 \quad 1 \quad$ U K.VAGSQIADYLHDR. I

2.3e-12 1 U K.VAGSQIADYLHDR. I

$6.7 e-09 \quad 1$

U K.GDINLEVMPESLLYK.T

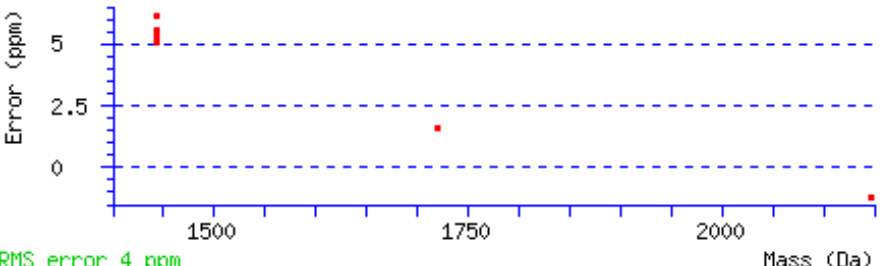




\section{MASCOT Search Results}

\section{Protein View: TP84_64}

$\begin{array}{ll}\text { Database: } & \text { TP84 } \\ \text { Score: } & 818 \\ \text { Nominal mass }\left(\mathbf{M}_{\mathbf{r}}\right): & 9330 \\ \text { Calculated pI: } & 6.89\end{array}$

Sequence similarity is available as an NCBI BLAST search of 36. against nr.

\section{Search parameters}

MS data file:

Enzyme:

: $\backslash F A \backslash 02-1$ uty2018\80202245zeb_czas25.raw

\section{Fixed modifications:}

Trypsin: cuts $\mathrm{C}$-term side of $\mathrm{KR}$ unless next residue is $\mathrm{P}$.

Variable modifications: Oxidation (M)

\section{Protein sequence coverage: $\mathbf{5 8 \%}$}

Matched peptides shown in bold red.

1 MVKYFVGSDV HFIAFDQNLF KHDKCFHIKR SDQIYSGITI YKVAGSOIAD

51 YLHDRIMNMY IKGDINLEVM PESLLYKTI

Unformatted sequence string: $\underline{79}$ residues (for pasting into other applications).

Sort peptides by $\odot$ Residue Number $\bigcirc$ Increasing Mass $\bigcirc$ Decreasing Mass

Show predicted peptides also

\begin{tabular}{|c|c|c|c|c|}
\hline Query & Start - End & Observed & $M r$ (expt) & $\mathrm{Mr}(\mathrm{calc})$ \\
\hline$\underbrace{\prime 3} 30268$ & $4-21$ & 1074.0378 & 2146.0610 & 2146.0524 \\
\hline $\mathbb{D}^{\prime 16136}$ & $43-55$ & 722.8606 & 1443.7067 & 1443.7106 \\
\hline$\nabla^{16137}$ & $43-55$ & 722.8615 & 1443.7084 & 1443.7106 \\
\hline$\mho^{\prime} 16138$ & $43-55$ & 482.2435 & 1443.7087 & 1443.7106 \\
\hline$\triangle^{\prime 16139}$ & $43-55$ & 482.2436 & 1443.7090 & 1443.7106 \\
\hline$\checkmark 22949$ & $63-77$ & 860.9448 & 1719.8751 & 1719.8753 \\
\hline$\checkmark 22950$ & $63-77$ & 860.9450 & 1719.8755 & 1719.8753 \\
\hline$\square^{\prime 2} \underline{2951}$ & $63-77$ & 860.9452 & 1719.8758 & 1719.8753 \\
\hline$\llbracket \underline{22952}$ & $63-77$ & 860.9458 & 1719.8771 & 1719.8753 \\
\hline 22953 & $63-77$ & 860.9466 & 1719.8787 & 1719.8753 \\
\hline
\end{tabular}

\begin{tabular}{rrr}
\multicolumn{1}{l|}{ Ppm } & M & Score \\
4.03 & 0 & 107 \\
-2.69 & 0 & 114 \\
-1.51 & 0 & 114 \\
-1.36 & 0 & 73 \\
-1.11 & 0 & 74 \\
-0.094 & 0 & 88 \\
0.14 & 0 & 86 \\
0.31 & 0 & 97 \\
1.08 & 0 & 93 \\
1.98 & 0 & 88
\end{tabular}

Expect Rank

2. $1 e-111$

3. $9 e-12$

$4 e-12 \quad 1$

$4.8 e-08$

$3.8 e-08 \quad 1$

1. $6 e-09$

2. $3 e-09 \quad 1$

1. $9 e-101$

$5.4 e-10 \quad 1$

1. $6 e-091$
U. Peptide

U K.YFVGSDVHFIAFDQNLFK . H

U K. VAGSQIADYLHDR. I

U K.VAGSQIADYLHDR. I

U K.VAGSQIADYLHDR. I

U K.VAGSQIADYLHDR. I

U K.GDINLEVMPESLLYK.T

U K. GDINLEVMPESLLYK. T

U K.GDINLEVMPESLLYK. T

U K. GDINLEVMPESLLYK.T

U K.GDINLEVMPESLLYK. T

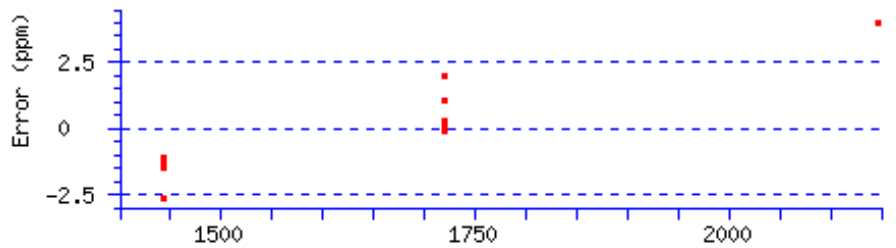

RIS error 1 ppm

Mass (Da) 


\title{
MASCOT Search Results
}

\section{Protein View: TP84_64}

\author{
Database: TP84 \\ Score: $\quad 474$ \\ Nominal mass $\left(\mathbf{M}_{\mathbf{r}}\right): 9330$ \\ Calculated pI: $\quad 6.89$
}

Sequence similarity is available as an NCBI BLAST search of 36 . against nr.

\section{Search parameters}

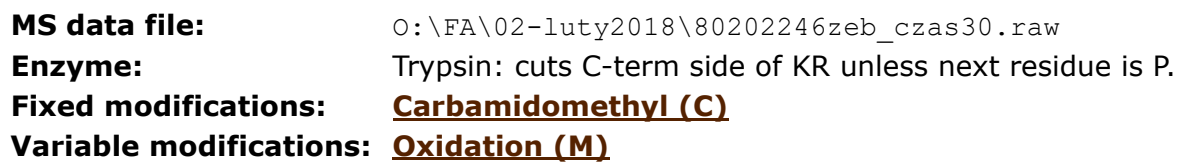

\section{Protein sequence coverage: $35 \%$}

Matched peptides shown in bold red.

1 MVKYFVGSDV HFIAFDQNLF KHDKCFHIKR SDQIYSGITI YKVAGSQIAD

51 YLHDRIMNMY IKGDINLEVM PESLLYKTI

Unformatted sequence string: $\underline{79}$ residues (for pasting into other applications).

Sort peptides by $\bullet$ Residue Number $\bigcirc$ Increasing Mass $\bigcirc$ Decreasing Mass

Show predicted peptides also

$\begin{array}{rc}\text { Query } & \text { Start }- \text { End } \\ \varangle \underline{22659} & 43-55 \\ \varangle \underline{22660} & 43-55 \\ \varangle \underline{2661} & 43-55 \\ \varangle \underline{31116} & 63-77 \\ \nabla \underline{31117} & 63-77\end{array}$

$\begin{array}{rrr}\text { Observed } & \text { Mr(expt) } & \text { Mr (calc) } \\ 722.8624 & 1443.7102 & 1443.7106 \\ 722.8631 & 1443.7117 & 1443.7106 \\ 722.8636 & 1443.7125 & 1443.7106 \\ 860.9450 & 1719.8755 & 1719.8753 \\ 860.9464 & 1719.8782 & 1719.8753\end{array}$

$\begin{array}{ccc}\text { ppm } & \text { M } & \text { Score } \\ -0.29 & 0 & 130 \\ 0.76 & 0 & 119 \\ 1.33 & 0 & 118 \\ 0.10 & 0 & 89 \\ 1.71 & 0 & 70\end{array}$

$\begin{array}{rc}\text { Expect } & \text { Rank } \\ 9.9 e-14 & 1 \\ 1.2 e-12 & 1 \\ 1.7 e-12 & \frac{1}{1} \\ 1.1 e-09 & 1 \\ 1 e-07 & \frac{1}{1}\end{array}$

U Peptide

Ü K. VAGSQIADYLHDR. I

U K.VAGSOIADYLHDR. I

U K.VAGSQIADYLHDR. I

$860.9464 \quad 1719.8782 \quad 1719.8753$

$1.710 \quad 70$

U K.GDINLEVMPESLLYK.T U K.GDINLEVMPESLLYK.T
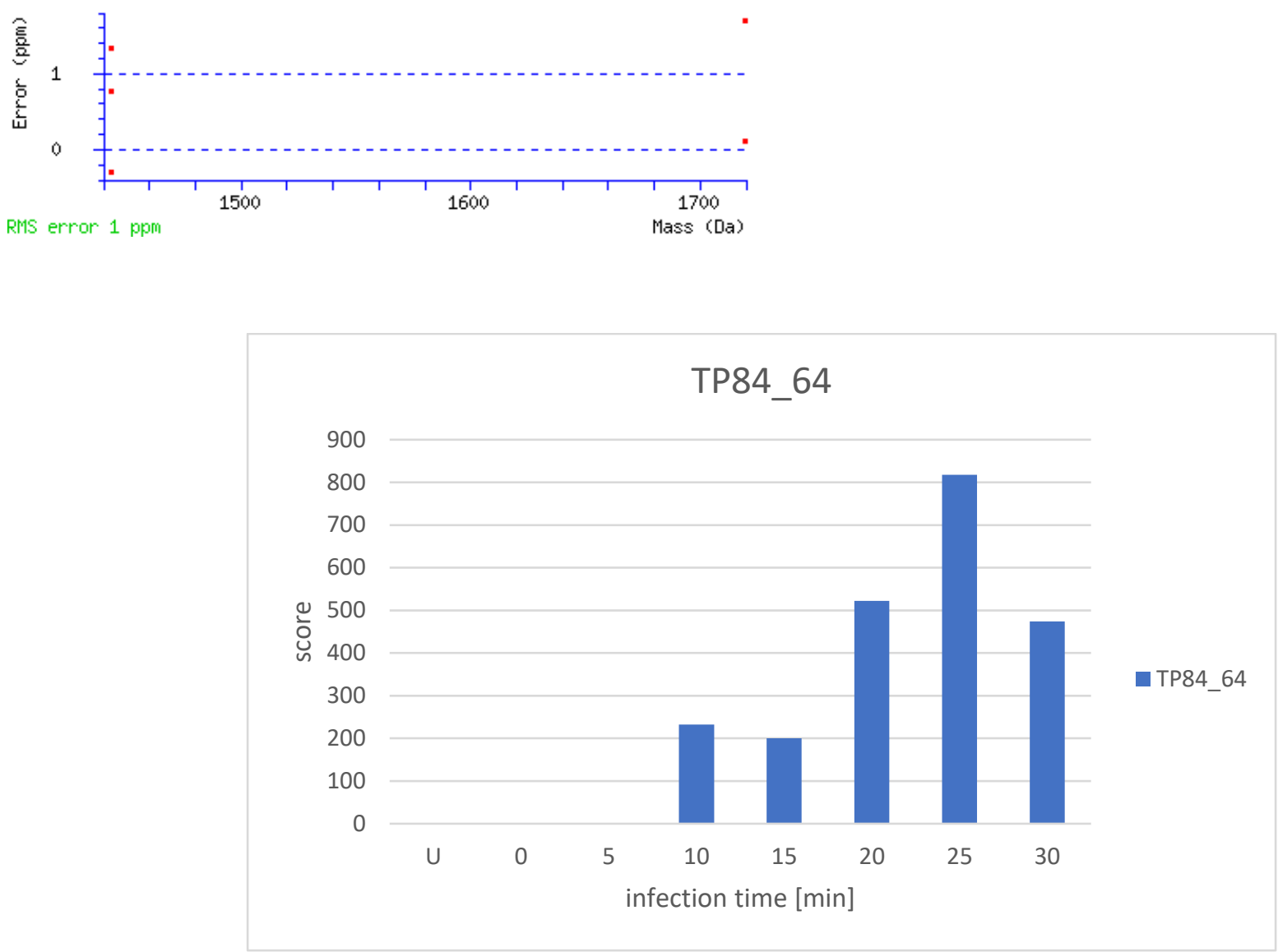


\title{
MASCOT Search Results
}

\section{Protein View: TP84_66}

\author{
Database: TP84 \\ Score: 343 \\ Nominal mass $\left(\mathbf{M}_{\mathbf{r}}\right)$ : 10393 \\ Calculated pI: $\quad 5.92$
}

Sequence similarity is available as an NCBI BLAST search of 38. against nr.

\section{Search parameters}

\section{MS data file: \\ Enzyme: \\ Fixed modifications: Carbamidomethyl (C) \\ Variable modifications: Oxidation $(M)$}

\section{Protein sequence coverage: $22 \%$}

Matched peptides shown in bold red.

1 MDIREEIYQA QKEREEFEKA RAASMPDEVE RKIVELMNDP RIQAEVMAII

51 IHLKKQKRKD IEILTIGLMV TNMARSVMEQ AAQTKQKGE

Unformatted sequence string: $\mathbf{8 9}$ residues (for pasting into other applications).

Sort peptides by $\bigcirc$ Residue Number $\bigcirc$ Increasing Mass $\bigcirc$ Decreasing Mass

Show predicted peptides also

\begin{tabular}{|c|c|}
\hline Query & Start - Er \\
\hline$\nearrow^{\prime} 11525$ & $22-31$ \\
\hline$\smile 11105$ & $76-85$ \\
\hline$\underline{11106}$ & $76-85$ \\
\hline$\nabla^{\prime 11107}$ & $76-85$ \\
\hline$\triangle^{\prime 11109}$ & $76-85$ \\
\hline$\nearrow^{\prime} 11110$ & $76-85$ \\
\hline 711111 & $76-$ \\
\hline
\end{tabular}

$\begin{array}{rrr}\text { Observed } & \text { Mr (expt) } & \text { Mr (calc) } \\ 552.7569 & 1103.4993 & 1103.4917 \\ 546.7746 & 1091.5346 & 1091.5281 \\ 546.7746 & 1091.5346 & 1091.5281 \\ 546.7746 & 1091.5347 & 1091.5281 \\ 546.7749 & 1091.5352 & 1091.5281 \\ 546.7749 & 1091.5352 & 1091.5281 \\ 546.7751 & 1091.5357 & 1091.5281\end{array}$

$\begin{array}{llr}\text { ppm } & \text { M } & \text { Score } \\ 6.90 & 0 & 67 \\ 5.95 & 0 & 58 \\ 6.01 & 0 & 60 \\ 6.03 & 0 & 61 \\ 6.48 & 0 & 60 \\ 6.50 & 0 & 61 \\ 6.98 & 0 & 54\end{array}$

Expect Rank $2 e-07 \quad 1$

U Peptide

$1.5 e-06 \quad 1$

SMPDEVER . K

9.1e-07 1 U R.SVMEQAAQTK.Q

8.7e-07 1 U R.SVMEQAAQTK.

$9.4 \mathrm{e}-07 \quad 1$ U R.SVMEQAAQTK.Q

$8.6 e-07 \quad 1$ U R.SVMEQAAQTK.Q

$3.8 e-06 \quad 1$ U R.SVMEQAAQTK.Q

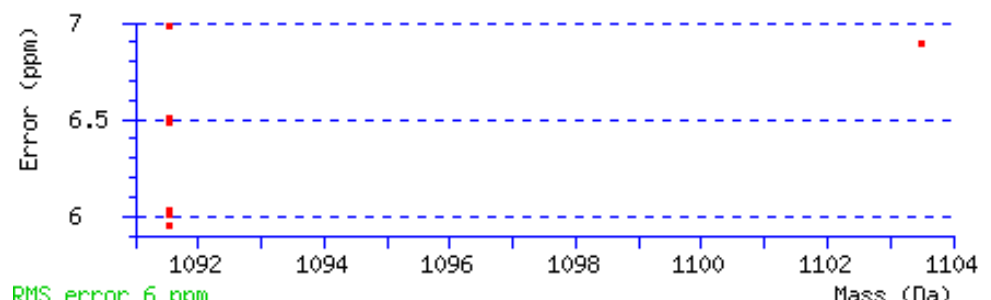

RMS error $6 \mathrm{ppm}$ Mass (Da) 


\title{
MASCOT Search Results
}

\section{Protein View: TP84_66}

\author{
Database: TP84 \\ Score: $\quad 458$ \\ Nominal mass $\left(\mathbf{M}_{\mathbf{r}}\right): 10393$ \\ Calculated pI: $\quad 5.92$
}

Sequence similarity is available as an NCBI BLAST search of 38. against nr.

\section{Search parameters}

\section{MS data file: \\ Enzyme: \\ Fixed modifications: Carbamidomethyl (C) \\ Variable modifications: Oxidation $(M)$}

\section{Protein sequence coverage: $22 \%$}

Matched peptides shown in bold red.

1 MDIREEIYQA QKEREEFEKA RAASMPDEVE RKIVELMNDP RIQAEVMAII

51 IHLKKQKRKD IEILTIGLMV TNMARSVMEQ AAQTKQKGE

Unformatted sequence string: $\mathbf{8 9}$ residues (for pasting into other applications).

Sort peptides by $\bigcirc$ Residue Number $\bigcirc$ Increasing Mass $\bigcirc$ Decreasing Mass

Show predicted peptides also

\begin{tabular}{|c|c|}
\hline Query & start \\
\hline$\smile^{\prime 7} \underline{196}$ & 22 \\
\hline$\triangle 7197$ & 22 \\
\hline$\triangleleft^{7} \mathbf{7 1 9 8}$ & 22 \\
\hline$\nearrow^{\prime 6903}$ & 76 \\
\hline$\checkmark 6904$ & 76 \\
\hline$\triangle \underline{6905}$ & 76 \\
\hline$\sigma^{6} \underline{906}$ & 76 \\
\hline
\end{tabular}

$\begin{array}{rrr}\text { Observed } & \text { Mr (expt) } & \text { Mr (calc) } \\ 552.7536 & 1103.4927 & 1103.4917 \\ 552.7538 & 1103.4931 & 1103.4917 \\ 552.7553 & 1103.4960 & 1103.4917 \\ 546.7717 & 1091.5289 & 1091.5281 \\ 546.7718 & 1091.5291 & 1091.5281 \\ 546.7719 & 1091.5292 & 1091.5281 \\ 546.7720 & 1091.5295 & 1091.5281\end{array}$

$\begin{array}{llc}\text { ppm } & \text { M } & \text { Score } \\ 0.90 & 0 & 88 \\ 1.26 & 0 & 77 \\ 3.94 & 0 & 80 \\ 0.79 & 0 & 77 \\ 0.93 & 0 & 71 \\ 1.06 & 0 & 71 \\ 1.30 & 0 & 71\end{array}$

Expect Rank

$1.5 e-091$

U Peptide

2. $1 e-08 \quad 1$

ASMPDEVER . K

9e-09 1 U R.AASMPDEVER.K

2.1e-08 1 U R.SVMEQAAQTK.Q

$7.5 e-08 \quad 1 \quad$ U $R . S V M E Q A A Q T K . Q$

$7.3 e-08 \quad 1 \quad$ U $R$ R.SVMEQAAQTK.Q $8 e-08 \quad 1$ U R.SVMEQAAQTK.Q

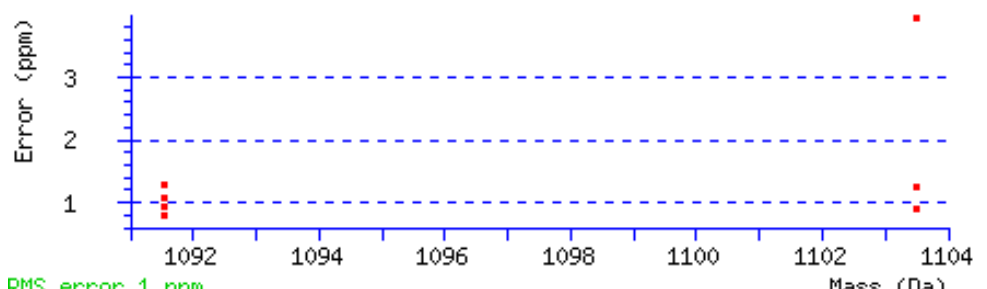

RMS error 1 ppm

Mass (Da) 


\title{
MASCOT Search Results
}

\section{Protein View: TP84_66}

$\begin{array}{ll}\text { Database: } & \text { TP84 } \\ \text { Score: } & 523 \\ \text { Nominal mass (Mr): } & 10393 \\ \text { Calculated pI: } & 5.92\end{array}$

Sequence similarity is available as an NCBI BLAST search of 38. against nr.

\section{Search parameters}

\author{
MS data file: \\ Enzyme: \\ $0: \backslash F A \backslash 02-1$ uty2018\80202246zeb_czas30.raw \\ Trypsin: cuts $\mathrm{C}$-term side of $\mathrm{KR}$ unless next residue is $\mathrm{P}$. \\ Carbamidomethyl (C) \\ Variable modifications: Oxidation (M)
}

\section{Protein sequence coverage: $22 \%$}

Matched peptides shown in bold red.

1 MDIREEIYQA QKEREEFEKA RAASMPDEVE RKIVELMNDP RIQAEVMAII

51 IHLKKQKRKD IEILTIGLMV TNMARSVMEQ AAQTKQKGE

Unformatted sequence string: $\mathbf{8 9}$ residues (for pasting into other applications).

Sort peptides by $\bigcirc$ Residue Number $\bigcirc$ Increasing Mass $\bigcirc$ Decreasing Mass

Show predicted peptides also

$\begin{array}{lc}\text { Query } & \text { Start - End } \\ \varangle \underline{9877} & 22-31 \\ \square \underline{9878} & 22-31 \\ \square \underline{9879} & 22-31 \\ \square \underline{9880} & 22-31 \\ \square \underline{9511} & 76-85 \\ \square \underline{9512} & 76-85 \\ \square \underline{9513} & 76-85 \\ \square \underline{9514} & 76-85\end{array}$

$\begin{array}{rrr}\text { Observed } & \text { Mr (expt) } & \text { Mr (calc) } \\ 552.7536 & 1103.4926 & 1103.4917 \\ 552.7537 & 1103.4928 & 1103.4917 \\ 552.7540 & 1103.4933 & 1103.4917 \\ 552.7541 & 1103.4937 & 1103.4917 \\ 546.7715 & 1091.5285 & 1091.5281 \\ 546.7717 & 1091.5289 & 1091.5281 \\ 546.7718 & 1091.5290 & 1091.5281 \\ 546.7723 & 1091.5301 & 1091.5281\end{array}$

$\begin{array}{llc}\text { ppm } & \text { M } & \text { Score } \\ 0.86 & 0 & 83 \\ 1.04 & 0 & 84 \\ 1.49 & 0 & 84 \\ 1.84 & 0 & 76 \\ 0.36 & 0 & 58 \\ 0.79 & 0 & 78 \\ 0.88 & 0 & 71 \\ 1.87 & 0 & 80\end{array}$

Expect Rank

5. 1 e- 091

3. $7 e-091$

3. $7 e-09 \quad 1$

$2.5 e-081$

$1.6 e-06 \quad 1$

$1.6 e-08$

$8.8 e-08$

$9.6 e-09 \quad 1$
U Peptide

R. AASMPDEVER . K

U R. AASMPDEVER. K

U R. AASMPDEVER. K

U R. AASMPDEVER. K

U R.SVMEQAAQTK.Q

U R. SVMEQAAQTK. Q

U R.SVMEOAAOTK.Q

U R.SVMEQAAQTK. Q

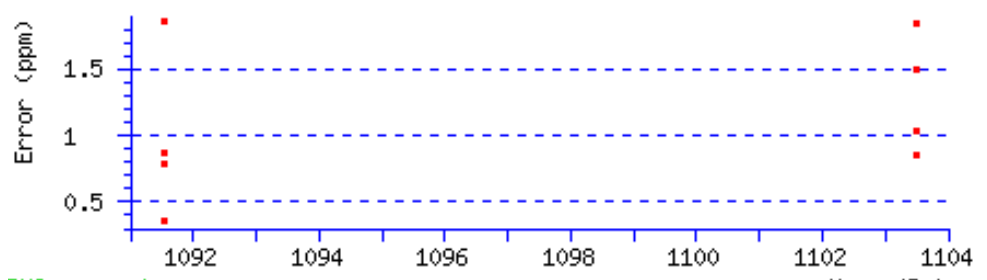

RMS error 1 ppm $M a s e(n a)$

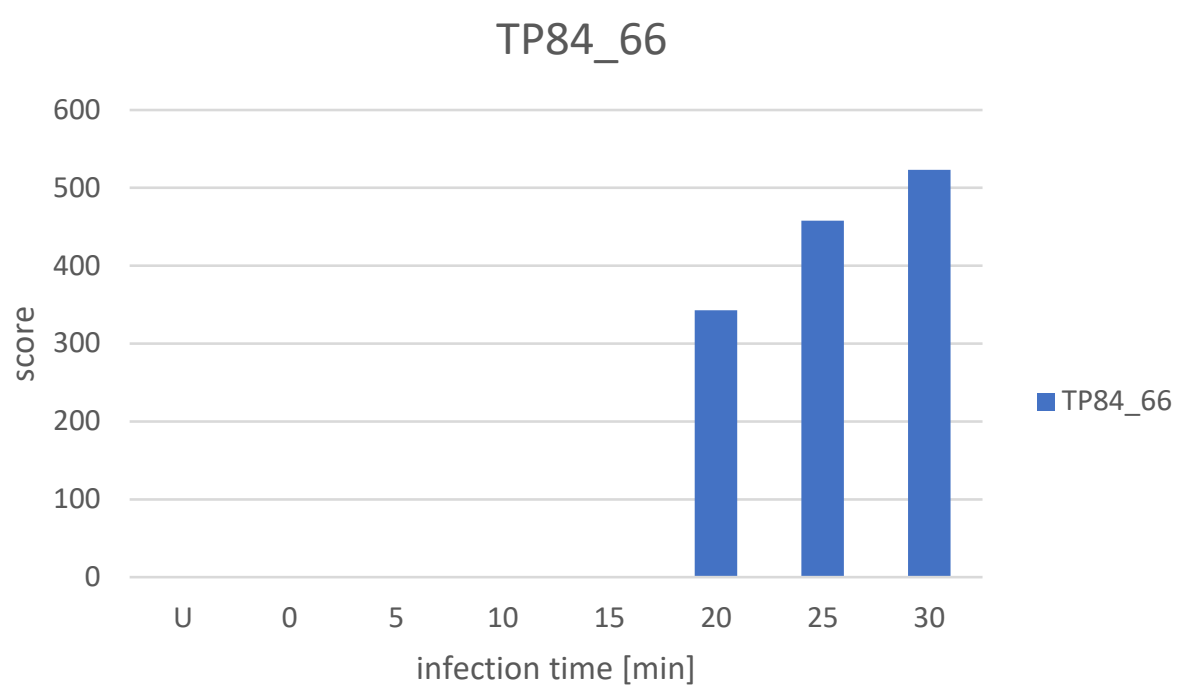




\title{
MASCOT Search Results
}

\section{Protein View: TP84_68}

\author{
Database: $\quad$ TP84 \\ Score: $\quad 715$ \\ Nominal mass $\left(M_{\mathbf{r}}\right): 38540$ \\ Calculated pI: $\quad 6.91$
}

Sequence similarity is available as an NCBI BLAST search of 40. against nr.

\section{Search parameters}

\author{
MS data file: \\ Enzyme: \\ $0: \backslash F A \backslash 02-l u t y 2018 \backslash 80202246 z e b \_c z a s 30 . r a w$ \\ Fixed modifications: \\ Trypsin: cuts C-term side of KR unless next residue is $\mathrm{P}$. \\ Carbamidomethyl (C) \\ Variable modifications: Oxidation (M)
}

\section{Protein sequence coverage: $\mathbf{2 7} \%$}

Matched peptides shown in bold red.
1 MEFKLYKVAG YEEAIMSLRM SKGKYFSWER AKKIQHLVYA VTDHRGFIAP
51 PQVYINNMRN LAAIEEDIKV DHQGKKGEIS GNYIRDVDEF KRLLALTLNN 101 AMGEHKHHTL MKYIDISFFT IGLHRGAQDD LDAHAIAFNN RITRYSTRLA 151 NIQETVLSEW YQDKIIPFEH AQHFVGIEWP MVIETDIGNF EYTPFGYIHE 201 NFNRISDENG LKKDVKRGLI PLSMASNALW KIDLFNLRYV YKMRSKLTKA 251 NPELKQGMEM LADQIEKHVP VFGPYFRYEL TDTGEWEHMN KVKTVTREEY 301 ELLKKIKKQM QQQGTVDVDL YRLQQEGE

Unformatted sequence string: $\mathbf{3 2 8}$ residues (for pasting into other applications).

Sort peptides by $\bullet$ Residue Number $\bigcirc$ Increasing Mass $\bigcirc$ Decreasing Mass

Show predicted peptides also

\begin{tabular}{|c|c|}
\hline Query & Start - End \\
\hline$\sigma^{\prime 18939}$ & $8-19$ \\
\hline$\sigma^{\prime 18940}$ & $8-19$ \\
\hline$\varangle 22934$ & $34-45$ \\
\hline$\checkmark 22935$ & $34-45$ \\
\hline$\nearrow^{2} \underline{2936}$ & $34-45$ \\
\hline$\sigma^{22938}$ & $34-45$ \\
\hline$\checkmark 27979$ & $46-59$ \\
\hline$\checkmark 25364$ & $93-106$ \\
\hline$\checkmark 31273$ & $126-141$ \\
\hline$\checkmark 36289$ & $149-164$ \\
\hline$\nabla^{3139}$ & $232-238$ \\
\hline
\end{tabular}

$\begin{array}{rrr}\text { Observed } & \text { Mr (expt) } & \text { Mr (calc) } \\ 669.8384 & 1337.6622 & 1337.6649 \\ 669.8388 & 1337.6631 & 1337.6649 \\ 363.6988 & 1450.7662 & 1450.7681 \\ 726.3913 & 1450.7680 & 1450.7681 \\ 726.3917 & 1450.7688 & 1450.7681 \\ 484.5971 & 1450.7694 & 1450.7681 \\ 810.4247 & 1618.8348 & 1618.8290 \\ 508.9451 & 1523.8136 & 1523.8130 \\ 864.4094 & 1726.8042 & 1726.8023 \\ 646.3260 & 1935.9563 & 1935.9578 \\ 445.7584 & 889.5022 & 889.5021\end{array}$

\begin{tabular}{rrr}
\multicolumn{1}{c}{ ppm } & M & Score \\
-1.98 & 0 & 111 \\
-1.37 & 0 & 77 \\
-1.30 & 0 & 52 \\
-0.034 & 0 & 86 \\
0.50 & 0 & 82 \\
0.89 & 0 & 64 \\
3.59 & 0 & 54 \\
0.40 & 0 & 64 \\
1.10 & 0 & 142 \\
-0.77 & 0 & 61 \\
0.10 & 0 & 52
\end{tabular}

$\begin{array}{rc}\text { Expect } & \text { Rank } \\ 7.9 e-12 & 1 \\ 1.9 e-08 & 1 \\ 6.7 e-06 & 1 \\ 2.3 e-09 & 1 \\ 6.8 e-09 & 1 \\ 4.2 e-07 & 1 \\ 3.9 e-06 & 1 \\ 3.9 e-07 & 1 \\ 6.3 e-15 & 1 \\ 8.1 e-07 & 1 \\ 6.2 e-06 & 1\end{array}$

U Peptide

U K. VAGYEEAIMSLR.M

U K.VAGYEEAIMSLR.M

U K.IQHLVYAVTDHR.G

U K.IQHLVYAVTDHR.G

U K.IQHLVYAVTDHR.G

U K.IQHLVYAVTDHR.G

U R.GFIAPPQVYINNMR . N

U R. LLALTLNNAMGEHK. H

U R.GAQDDLDAHAIAFNNR. I

U R. LANIQETVLSEWYQDK. I

U K. IDLFNLR.Y

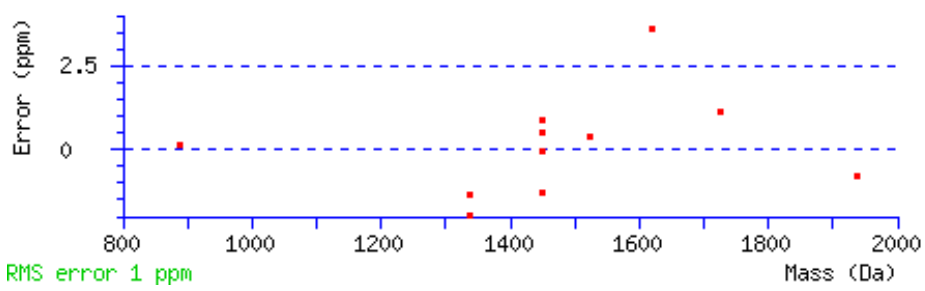




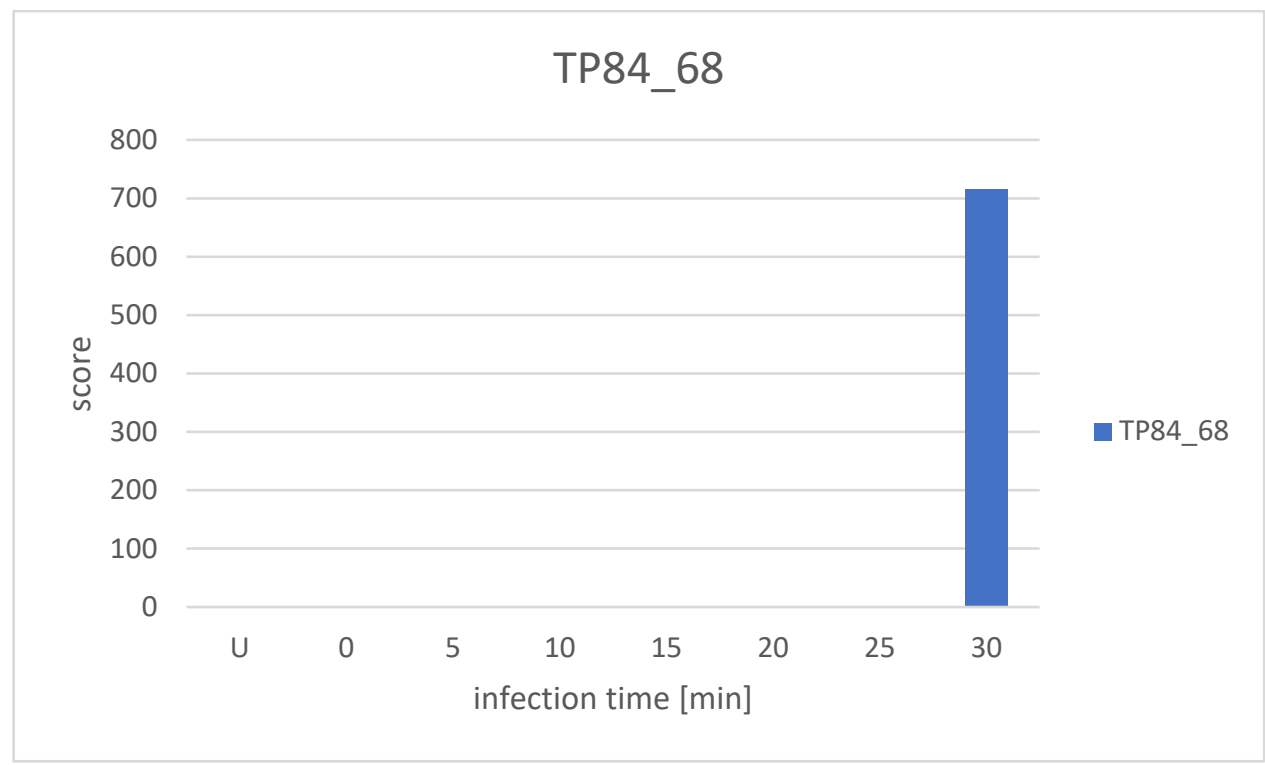




\title{
MASCOT Search Results
}

\section{Protein View: TP84_69}

\author{
Database: TP84 \\ Score: $\quad 878$ \\ Nominal mass (Mr): 20065 \\ Calculated pI: $\quad 5.67$
}

Sequence similarity is available as an NCBI BLAST search of 41. against nr.

\section{Search parameters}

\author{
MS data file: \\ Enzyme: \\ Fixed modifications: \\ Variable modifications: Oxidation (M)
}

\section{Protein sequence coverage: $15 \%$}

Matched peptides shown in bold red.

\footnotetext{
1 MVKVDERTIN LSDLFQKQKM LTDYVWQKHN IKKADLEKEK AAIICELWET

51 ANELKSDGFK YWTDKKCDRE KTLEEIVDML HFYLQIGNIL GVVYEHYWIE 101 RRDTILDQIM AINWSLLMMD GPLTWAVSFA QYRGLVRMLG FDWDQDIIPA 151 YNRKFQENIA RQQRGY
}

Unformatted sequence string: 166 residues (for pasting into other applications).
Show predicted peptides also

\begin{tabular}{|c|c|c|c|c|}
\hline Query & Start - End & Observed & $M r(\exp t)$ & $\mathrm{Mr}(\mathrm{calc})$ \\
\hline$\sigma^{\prime} 12657$ & $8-17$ & 589.8257 & 1177.6368 & 1177.6343 \\
\hline$\sigma^{\prime 12658}$ & $8-17$ & 589.8260 & 1177.6374 & 1177.6343 \\
\hline$\sigma^{\prime 12659}$ & $8-17$ & 589.8261 & 1177.6376 & 1177.6343 \\
\hline$\sigma^{\prime} 12660$ & $8-17$ & 589.8262 & 1177.6379 & 1177.6343 \\
\hline$\sigma^{\prime} 36468$ & $138-153$ & 977.4587 & 1952.9028 & 1952.9091 \\
\hline$\checkmark 36469$ & $138-153$ & 977.4587 & 1952.9029 & 1952.9091 \\
\hline$\sigma^{\prime 36470}$ & $138-153$ & 977.4591 & 1952.9036 & 1952.9091 \\
\hline$\sigma^{\prime 36471}$ & $138-153$ & 977.4598 & 1952.9051 & 1952.9091 \\
\hline$\sigma^{\prime 36472}$ & $138-153$ & 977.4612 & 1952.9078 & 1952.9091 \\
\hline$\sigma^{\prime} \underline{36473}$ & $138-153$ & 977.4613 & 1952.9081 & 1952.9091 \\
\hline
\end{tabular}

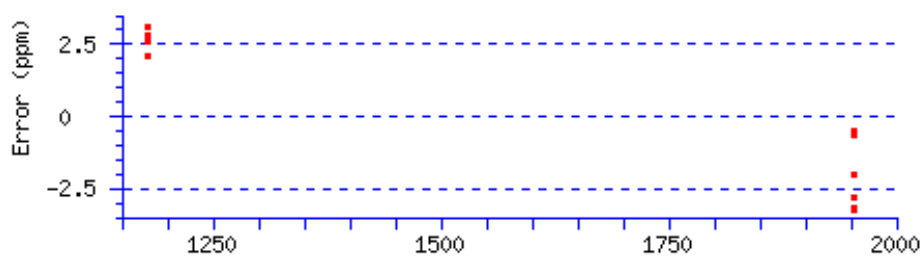

RMS error 2 ppm

\begin{tabular}{|c|c|c|c|c|c|c|}
\hline ppm & M & Score & Expect & Rank & $\mathrm{U}$ & Peptide \\
\hline 2.12 & $\ddot{0}$ & 52 & $5.6 e-06$ & 1 & $\dddot{\mathrm{U}}$ & R.TINLSDLFQK.Q \\
\hline 2.62 & 0 & 69 & $1.4 e-07$ & 1 & $\mathrm{U}$ & R.TINLSDLFQK.Q \\
\hline 2.79 & 0 & 63 & $5.3 e-07$ & 1 & $\mathrm{U}$ & R.TINLSDLFQK.Q \\
\hline 3.12 & 0 & 63 & $5.3 e-07$ & 1 & $\mathrm{U}$ & R.TINLSDLFQK.Q \\
\hline-3.23 & 0 & 128 & $1.6 e-13$ & 1 & $\mathrm{U}$ & R.MLGFDWDQDI IPAYNR. K \\
\hline-3.15 & 0 & 113 & $5.2 e-12$ & 1 & $\mathrm{U}$ & R.MLGFDWDQDI IPAYNR.K \\
\hline-2.82 & 0 & 130 & $9.2 e-14$ & 1 & $\mathrm{U}$ & R.MLGFDWDQDI IPAYNR.K \\
\hline-2.01 & 0 & 134 & $4.1 e-14$ & 1 & $\mathrm{U}$ & R.MLGFDWDQDI IPAYNR.K \\
\hline-0.64 & 0 & 128 & $1.6 e-13$ & 1 & $\mathrm{U}$ & R.MLGFDWDQDI I PAYNR.K \\
\hline-0.51 & 0 & 115 & $3.1 e-12$ & 1 & $\mathrm{U}$ & R.MLGFDWDQDI IPAYNR.K \\
\hline
\end{tabular}


TP84_69

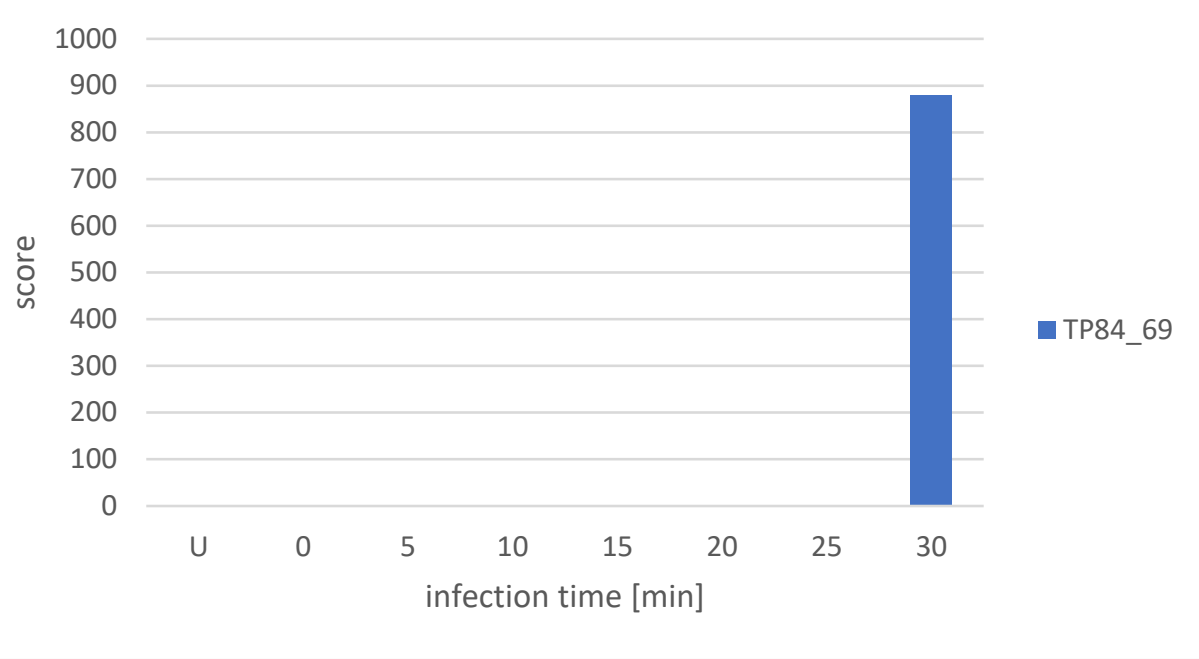




\title{
MASCOT Search Results
}

\section{Protein View: TP84_79}

$\begin{array}{ll}\text { Database: } & \text { TP84 } \\ \text { Score: } & 329 \\ \text { Nominal mass (Mr): } & 13449 \\ \text { Calculated pI: } & 7.82\end{array}$

Sequence similarity is available as an NCBI BLAST search of 52 . against nr.

\section{Search parameters}

\author{
MS data file: $\quad 0: \backslash F A \backslash 02-1$ uty2018\80202243zeb_czas15.raw \\ Enzyme: $\quad$ Trypsin: cuts C-term side of KR unless next residue is $P$. \\ Fixed modifications: Carbamidomethyl (C) \\ Variable modifications: Oxidation (M)
}

\section{Protein sequence coverage: $\mathbf{2 3} \%$}

Matched peptides shown in bold red.

1 MAKTTTQFQY HSGVGDATSY IHTSEDYMNV YVTVELGRVG TWETEAWCKL

51 ALQRYENGAW KTIATAQGYA ATGQNLNRTF SNISNVMEKP MRVKVDLYAN

101 SSYSDYVQTV YTKQWIR

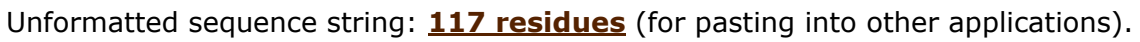

Sort peptides by $\bigcirc$ Residue Number $\bigcirc$ Increasing Mass $\bigcirc$ Decreasing Mass

Show predicted peptides also

$\begin{array}{rc}\text { Query } & \text { Start }- \text { End } \\ \nabla^{\prime} 11679 & 39-49 \\ \nabla^{\prime} \underline{19787} & 62-78 \\ \nabla^{\prime} \underline{19788} & 62-78 \\ \nabla^{\prime} \underline{19789} & 62-78 \\ \nabla^{\prime} \underline{19790} & 62-78\end{array}$

$\begin{array}{rrr}\text { Observed } & \text { Mr (expt) } & \text { Mr (calc) } \\ 683.8082 & 1365.6018 & 1365.6023 \\ 583.9655 & 1748.8746 & 1748.8805 \\ 875.4461 & 1748.8776 & 1748.8805 \\ 583.9668 & 1748.8785 & 1748.8805 \\ 875.4488 & 1748.8830 & 1748.8805\end{array}$

ppm M Score Expect Rank U Peptide

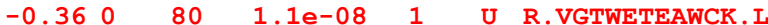

$-3.420 \quad 60 \quad 9.8 e-07 \quad 3 \quad$ U $\quad$ K.TIATAQGYAATGQNLNR.T

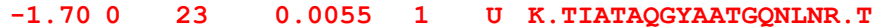

$\begin{array}{llllll}-1.160 & 61 & 8.1 e-07 & 1 & \text { U } & \text { K.TIATAQGYAATGQNLNR.T }\end{array}$

$\checkmark \underline{19790} \quad 62-78$

875.4488

$1.420 \quad 157$

$1.8 e-16 \quad 1$

U K. TIATAQGYAATGQNLNR. T

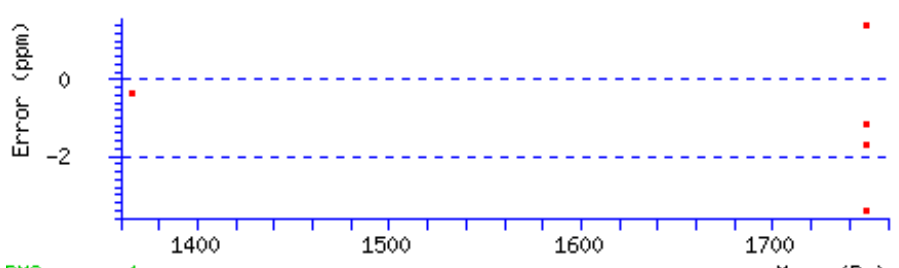

RMS error $1 \mathrm{ppm}$ Mass (Da) 


\title{
MASCOT Search Results
}

\section{Protein View: TP84_79}

\author{
Database: TP84 \\ Score: $\quad 1140$ \\ Nominal mass $\left(\mathbf{M}_{\mathbf{r}}\right)$ : 13449 \\ Calculated pI: $\quad 7.82$
}

Sequence similarity is available as an NCBI BLAST search of 52 . against nr.

\section{Search parameters}

MS data file:

Enzyme:

Fixed modifications:

Variable mo

\section{Protein sequence coverage: $26 \%$}

Matched peptides shown in bold red.

1 MAKTTTQFQY HSGVGDATSY IHTSEDYMNV YVTVELGRVG TWETEAWCKL

51 ALQRYENGAW KTIATAQGYA ATGQNLNRTF SNISNVMEKP MRVKVDLYAN 101 SSYSDYVQTV YTKQWIR

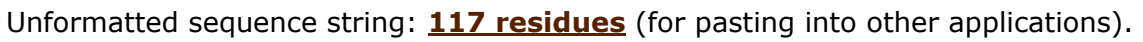

Sort peptides by $\odot$ Residue Number $\bigcirc$ Increasing Mass $\bigcirc$ Decreasing Mass

Show predicted peptides also

\begin{tabular}{|c|c|c|c|c|}
\hline Query & Start - End & Observed & $M r(\exp t)$ & $\operatorname{Mr}(\mathrm{calc})$ \\
\hline$\sigma^{\prime 32668}$ & $62-78$ & 875.4505 & 1748.8865 & 1748.8805 \\
\hline$\overline{32669}$ & $62-78$ & 875.4509 & 1748.8873 & 1748.8805 \\
\hline$\sigma^{\prime 32670}$ & $62-78$ & 583.9697 & 1748.8874 & 1748.8805 \\
\hline$\sigma^{\prime 32671}$ & $62-78$ & 875.4511 & 1748.8875 & 1748.8805 \\
\hline$\overline{32672}$ & $62-78$ & 583.9701 & 1748.8886 & 1748.8805 \\
\hline$\sigma^{\prime} \underline{32675}$ & $62-78$ & 583.9704 & 1748.8893 & 1748.8805 \\
\hline$\checkmark 32676$ & $62-78$ & 583.9704 & 1748.8895 & 1748.8805 \\
\hline$\checkmark x 32678$ & $62-78$ & 875.4531 & 1748.8916 & 1748.8805 \\
\hline$\forall 229974$ & $79-92$ & 827.4096 & 1652.8046 & 1652.8014 \\
\hline$\varangle \underline{29975}$ & $79-92$ & 551.9442 & 1652.8107 & 1652.8014 \\
\hline$\checkmark \overline{29976}$ & $79-92$ & 551.9443 & 1652.8112 & 1652.8014 \\
\hline$\varangle 29977$ & $79-92$ & 827.4134 & 1652.8123 & 1652.8014 \\
\hline$\varpi^{\prime 30414}$ & $79-92$ & 557.2755 & 1668.8047 & 1668.7963 \\
\hline$\sigma^{\prime 30415}$ & $79-92$ & 557.2762 & 1668.8067 & 1668.7963 \\
\hline
\end{tabular}

Ppm M Score Expect Rank U Peptide

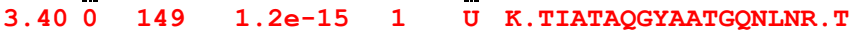

$3.850 \quad 155 \quad 3.2 e-16 \quad \cdots \quad$ U $\quad$ K.TIATAQGYAATGQNLNR.T

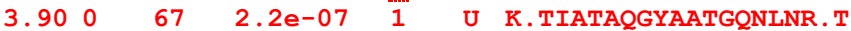

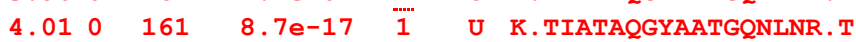

$4.620 \quad 63 \quad 5.5 e-07 \quad 1 \quad$ U $\quad$ K. TIATAQGYAATGQNLNR. T

$5.020 \quad 51 \quad 8 e-06 \quad 1$ U K. TIATAQGYAATGQNLNR. T

$\begin{array}{llllll}5.10 & 70 & 1 e-07 & 1 & \text { U } & \text { K. TIATAQGYAATGQNLNR.T }\end{array}$

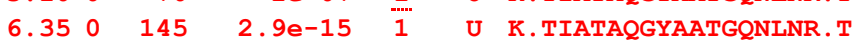

$\begin{array}{llllll}1.930 & 97 & 1.9 e-10 & 1 & \text { U } & \text { R.TFSNISNVMEKPMR.V }\end{array}$

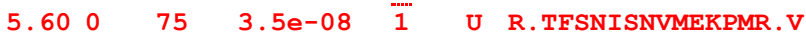

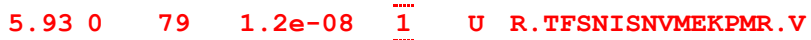

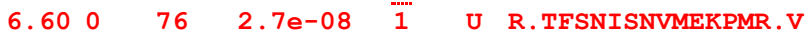

$\begin{array}{llllll}5.030 & 60 & 9.9 e-07 & 1 & \text { U } & \text { R.TFSNISNVMEKPMR.V }\end{array}$ + Oxidation (M)

$6.21062 \quad 5.8 e-07 \quad 1 \quad$ U $\quad$ R.TFSNISNVMEKPMR.V

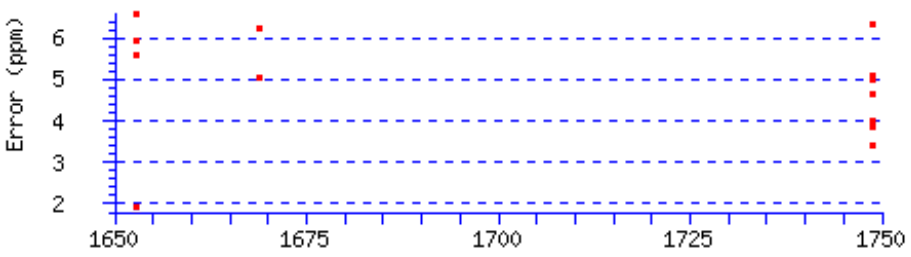

RIS error 4 ppm Mass (Da) 


\section{MASCOT Search Results}

\section{Protein View: TP84_79}

$\begin{array}{ll}\text { Database: } & \text { TP84 } \\ \text { Score: } & 2462 \\ \text { Nominal mass (Mr): } & 13449 \\ \text { Calculated pI: } & 7.82\end{array}$

Sequence similarity is available as an NCBI BLAST search of $\mathbf{5 2}$. against nr.

\section{Search parameters}

MS data file:

Enzyme:

Fixed modifications:

Variable modifications: Oxidation (M)

\section{Protein sequence coverage: $52 \%$}

Matched peptides shown in bold red.

1 MAKTTTQFQY HSGVGDATSY IHTSEDYMNV YVTVELGRVG TWETEAWCKL

51 ALQRYENGAW KTIATAQGYA ATGQNLNRTF SNISNVMEKP MRVKVDLYAN 101 SSYSDYVQTV YTKQWIR

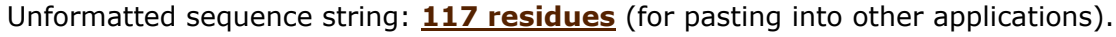

Sort peptides by $\odot$ Residue Number $\bigcirc$ Increasing Mass $\bigcirc$ Decreasing Mass

Show predicted peptides also

\begin{tabular}{|c|c|c|c|c|}
\hline Query & Start - End & Observed & $M r(\exp t)$ & $\mathrm{Mr}$ (calc) \\
\hline$\nabla^{\prime 14118}$ & $39-49$ & 683.8071 & 1365.5996 & 1365.6023 \\
\hline$\overline{14119}$ & $39-49$ & 683.8074 & 1365.6002 & 1365.6023 \\
\hline$\nabla^{14120}$ & $39-49$ & 683.8076 & 1365.6007 & 1365.6023 \\
\hline$\sigma^{\prime 14121}$ & $39-49$ & 683.8086 & 1365.6026 & 1365.6023 \\
\hline$\overline{23493}$ & $62-78$ & 875.4418 & 1748.8690 & 1748.8805 \\
\hline$\checkmark \underline{23495}$ & $62-78$ & 583.9655 & 1748.8746 & 1748.8805 \\
\hline 23496 & $62-78$ & 875.4455 & 1748.8764 & 1748.8805 \\
\hline$\checkmark 23497$ & $62-78$ & 875.4459 & 1748.8773 & 1748.8805 \\
\hline$\checkmark 23498$ & $62-78$ & 583.9665 & 1748.8776 & 1748.8805 \\
\hline$\checkmark 23499$ & $62-78$ & 875.4467 & 1748.8789 & 1748.8805 \\
\hline$\checkmark \underline{23500}$ & $62-78$ & 875.4469 & 1748.8792 & 1748.8805 \\
\hline$\checkmark \underline{23501}$ & $62-78$ & 875.4470 & 1748.8794 & 1748.8805 \\
\hline$\checkmark 23502$ & $62-78$ & 875.4472 & 1748.8798 & 1748.8805 \\
\hline$\square^{23} 3503$ & $62-78$ & 875.4472 & 1748.8798 & 1748.8805 \\
\hline$\checkmark 23504$ & $62-78$ & 875.4475 & 1748.8804 & 1748.8805 \\
\hline$\checkmark \underline{23505}$ & $62-78$ & 583.9674 & 1748.8804 & 1748.8805 \\
\hline$\checkmark 23506$ & $62-78$ & 583.9674 & 1748.8805 & 1748.8805 \\
\hline$\checkmark 23507$ & $62-78$ & 875.4476 & 1748.8807 & 1748.8805 \\
\hline$\checkmark \underline{23508}$ & $62-78$ & 583.9677 & 1748.8814 & 1748.8805 \\
\hline$\checkmark 23509$ & $62-78$ & 583.9678 & 1748.8817 & 1748.8805 \\
\hline $\mathbb{U}^{23} 3511$ & $62-78$ & 583.9680 & 1748.8823 & 1748.8805 \\
\hline$ð 21073$ & $79-92$ & 827.4067 & 1652.7989 & 1652.8014 \\
\hline$\checkmark 21074$ & $79-92$ & 827.4070 & 1652.7994 & 1652.8014 \\
\hline$\varangle \underline{21075}$ & $79-92$ & 551.9417 & 1652.8033 & 1652.8014 \\
\hline$\varangle \underline{21462}$ & $79-92$ & 557.2732 & 1668.7977 & 1668.7963 \\
\hline$\underline{31052}$ & $95-113$ & 1108.5281 & 2215.0416 & 2215.0321 \\
\hline
\end{tabular}

\begin{tabular}{|c|c|c|c|c|c|c|}
\hline ppm & $\mathbf{M}$ & Score & Expect & Rank & $\mathrm{U}$ & Peptide \\
\hline-1.97 & $\dddot{0}$ & 90 & $9.8 e-10$ & 1 & $\dddot{\mathrm{U}}$ & R.VGTWETEAWCK. L \\
\hline-1.56 & 0 & 95 & $3.2 e-10$ & 1 & $\mathrm{U}$ & R. VGTWETEAWCK. L \\
\hline-1.23 & 0 & 94 & $4 e-10$ & 1 & $\mathrm{U}$ & R.VGTWETEAWCK. L \\
\hline 0.22 & 0 & 88 & $1.8 e-09$ & 1 & $\mathrm{U}$ & R.VGTWETEAWCK. L \\
\hline-6.62 & 0 & 113 & $5.6 e-12$ & 1 & $\mathrm{U}$ & K. TIATAQGYAATGQNLNR. T \\
\hline-3.42 & 0 & 58 & $1.6 e-06$ & 1 & $\mathrm{U}$ & K. TIATAQGYAATGQNLNR.T \\
\hline-2.37 & 0 & 112 & $5.9 e-12$ & 1 & $\mathrm{U}$ & K. TIATAQGYAATGQNLNR.T \\
\hline-1.85 & 0 & 93 & $5 e-10$ & 1 & $\mathrm{U}$ & K. TIATAQGYAATGQNLNR.T \\
\hline-1.67 & 0 & 68 & $1.6 e-07$ & 1 & $\mathrm{U}$ & K. TIATAQGYAATGQNLNR.T \\
\hline-0.91 & 0 & 152 & $6.7 e-16$ & 1 & $\mathrm{U}$ & K. TIATAQGYAATGQNLNR. T \\
\hline-0.74 & 0 & 152 & $6.5 e-16$ & 1 & $\mathrm{U}$ & K. TIATAQGYAATGQNLNR.T \\
\hline-0.64 & 0 & 158 & $1.6 e-16$ & 1 & $\mathrm{U}$ & K. TIATAQGYAATGQNLNR. T \\
\hline-0.42 & 0 & 161 & $7.7 e-17$ & 1 & $\mathrm{U}$ & K. TIATAQGYAATGQNLNR. T \\
\hline-0.42 & 0 & 161 & $8.4 e-17$ & 1 & $\mathrm{U}$ & K. TIATAQGYAATGQNLNR.T \\
\hline-0.098 & 0 & 153 & $5.2 e-16$ & 1 & $\mathrm{U}$ & K. TIATAQGYAATGQNLNR.T \\
\hline-0.061 & 0 & 90 & $1 e-09$ & 1 & $\mathrm{U}$ & K. TIATAQGYAATGQNLNR.T \\
\hline-0.027 & 0 & 78 & $1.6 e-08$ & 1 & $\mathrm{U}$ & K.TIATAQGYAATGQNLNR.T \\
\hline 0.074 & 0 & 154 & $3.6 e-16$ & 1 & $\mathrm{U}$ & K. TIATAQGYAATGQNLNR.T \\
\hline 0.49 & 0 & 88 & $1.7 e-09$ & 1 & $\mathrm{U}$ & K. TIATAQGYAATGQNLNR.T \\
\hline 0.68 & 0 & 83 & $5.1 e-09$ & 1 & $\mathrm{U}$ & K. TIATAQGYAATGQNLNR.T \\
\hline 0.99 & 0 & 83 & $4.7 e-09$ & 1 & $\mathrm{U}$ & K. TIATAQGYAATGQNLNR . T \\
\hline-1.50 & 0 & 103 & $5.4 e-11$ & 1 & $\mathrm{U}$ & R.TFSNISNVMEKPMR.V \\
\hline-1.22 & 0 & 113 & $5 e-12$ & 1 & $\mathrm{U}$ & R. TFSNISNVMEKPMR.V \\
\hline 1.16 & 0 & 90 & $9.5 e-10$ & 1 & $\mathrm{U}$ & R. TFSNISNVMEKPMR.V \\
\hline 0.82 & 0 & 61 & $7.5 e-07$ & 1 & $\mathrm{U}$ & $\begin{array}{l}\text { R. TFSNISNVMEKPMR. V + } \\
\text { Oxidation (M) }\end{array}$ \\
\hline 4 & & 97 & $1 e-10$ & 1 & U & K.VDLYANSSYSDYVQTVYTK \\
\hline
\end{tabular}

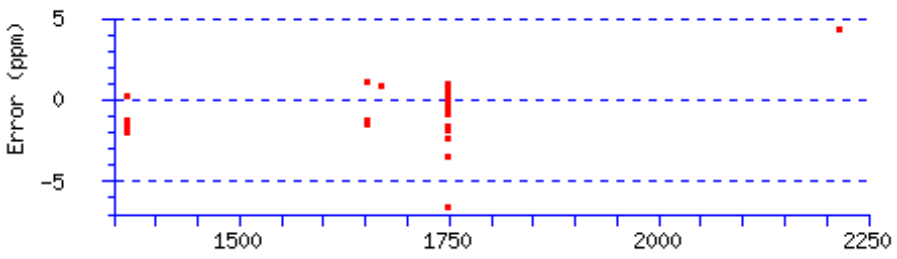




\title{
MASCOT Search Results
}

\section{Protein View: TP84_79}

\author{
Database: TP84 \\ Score: $\quad 3398$ \\ Nominal mass $\left(\mathbf{M}_{\mathbf{r}}\right)$ : 13449 \\ Calculated pI: $\quad 7.82$
}

Sequence similarity is available as an NCBI BLAST search of $\mathbf{5 2}$. against nr.

\section{Search parameters}

MS data file:

Enzyme:

Fixed modifications:

Variable modifications: Oxidation $(M)$

\section{Protein sequence coverage: $\mathbf{5 8 \%}$}

Matched peptides shown in bold red.

1 MAKTTTQFQY HSGVGDATSY IHTSEDYMNV YVTVELGRVG TWETEAWCKL

51 ALQRYENGAW KTIATAQGYA ATGQNLNRTF SNISNVMEKP MRVKVDLYAN 101 SSYSDYVQTV YTKQWIR

Unformatted sequence string: 1117 residues (for pasting into other applications).

Sort peptides by $\bullet$ Residue Number $\bigcirc$ Increasing Mass $\bigcirc$ Decreasing Mass

Show predicted peptides also

\begin{tabular}{|c|c|c|c|c|}
\hline Query & Start - End & Observed & $M r$ (expt) & $\mathrm{Mr}$ (calc) \\
\hline$\varpi^{\prime 19924}$ & $39-49$ & 683.8054 & 1365.5963 & 1365.6023 \\
\hline$\checkmark 19925$ & $39-49$ & 683.8055 & 1365.5964 & 1365.6023 \\
\hline$\checkmark 19926$ & $39-49$ & 683.8060 & 1365.5975 & 1365.6023 \\
\hline$\checkmark 19927$ & $39-49$ & 683.8066 & 1365.5987 & 1365.6023 \\
\hline$\checkmark 19928$ & $39-49$ & 683.8069 & 1365.5992 & 1365.6023 \\
\hline$\mho^{\prime} 19929$ & $39-49$ & 683.8071 & 1365.5995 & 1365.6023 \\
\hline$\checkmark 19930$ & $39-49$ & 683.8072 & 1365.5998 & 1365.6023 \\
\hline$ه 19931$ & $39-49$ & 683.8073 & 1365.6001 & 1365.6023 \\
\hline$\checkmark 19932$ & $39-49$ & 683.8073 & 1365.6001 & 1365.6023 \\
\hline$\sigma^{\prime 19933}$ & $39-49$ & 683.8075 & 1365.6005 & 1365.6023 \\
\hline$\underbrace{\prime 2584}$ & $55-61$ & 434.2032 & 866.3919 & 866.3922 \\
\hline$\underbrace{\prime 31814}$ & $62-78$ & 875.4442 & 1748.8739 & 1748.8805 \\
\hline 31816 & $62-78$ & 875.4449 & 1748.8753 & 1748.8805 \\
\hline $\mathbb{J}^{\prime} 31817$ & $62-78$ & 875.4455 & 1748.8764 & 1748.8805 \\
\hline$\varpi^{\prime 3} \underline{1818}$ & $62-78$ & 875.4460 & 1748.8775 & 1748.8805 \\
\hline $\mathbb{D}^{\prime 31819}$ & $62-78$ & 875.4461 & 1748.8777 & 1748.8805 \\
\hline$\varpi^{\prime 31820}$ & $62-78$ & 875.4462 & 1748.8778 & 1748.8805 \\
\hline$\nearrow^{\prime} 31821$ & $62-78$ & 875.4464 & 1748.8781 & 1748.8805 \\
\hline$\checkmark \underline{31822}$ & $62-78$ & 875.4464 & 1748.8782 & 1748.8805 \\
\hline “31823 & $62-78$ & 875.4464 & 1748.8783 & 1748.8805 \\
\hline $\mathbb{d}^{\prime 31824}$ & $62-78$ & 583.9669 & 1748.8788 & 1748.8805 \\
\hline$\square^{\prime 3} \underline{1825}$ & $62-78$ & 875.4468 & 1748.8790 & 1748.8805 \\
\hline$\underbrace{\prime} 31826$ & $62-78$ & 875.4470 & 1748.8794 & 1748.8805 \\
\hline ه'31827 & $62-78$ & 583.9674 & 1748.8804 & 1748.8805 \\
\hline$\underbrace{\prime 31828}$ & $62-78$ & 583.9674 & 1748.8805 & 1748.8805 \\
\hline$\sigma^{\prime 3} 31829$ & $62-78$ & 875.4476 & 1748.8806 & 1748.8805 \\
\hline$\square^{\prime 31830}$ & $62-78$ & 583.9679 & 1748.8818 & 1748.8805 \\
\hline$\underbrace{\prime 31831}$ & $62-78$ & 583.9680 & 1748.8822 & 1748.8805 \\
\hline$\nearrow^{28828}$ & $79-92$ & 827.4054 & 1652.7963 & 1652.8014 \\
\hline$\triangle 28829$ & $79-92$ & 827.4055 & 1652.7965 & 1652.8014 \\
\hline$\varangle 28830$ & $79-92$ & 827.4057 & 1652.7968 & 1652.8014 \\
\hline$\varangle 28831$ & $79-92$ & 827.4061 & 1652.7977 & 1652.8014 \\
\hline$ð^{28832}$ & $79-92$ & 827.4072 & 1652.7998 & 1652.8014 \\
\hline$\varpi^{28833}$ & $79-92$ & 551.9408 & 1652.8005 & 1652.8014 \\
\hline$\nearrow^{\prime 28834}$ & $79-92$ & 551.9409 & 1652.8009 & 1652.8014 \\
\hline ¿29331 & $79-92$ & 557.2724 & 1668.7955 & 1668.7963 \\
\hline$\varangle \underline{29332}$ & $79-92$ & 835.4070 & 1668.7993 & 1668.7963 \\
\hline$\triangle \underline{40422}$ & $95-113$ & 1108.5224 & 2215.0302 & 2215.0321 \\
\hline$\square^{\prime 4} \underline{0423}$ & $95-113$ & 1108.5322 & 2215.0498 & 2215.0321 \\
\hline
\end{tabular}

Ppm M Score Expect Rank U Peptide

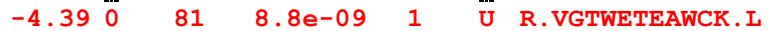

$-4.330$

$-3.520$

$-2.660$

$-2.310$

$-2.050$

$-1.870$

$-1.660$

$-1.650$

$-1.370$

$-0.390$

$-3.800$

$-2.990$

$-2.380$

$-1.730$

$-1.600$

$-1.570$

$-1.370$

$-1.340$

$-1.260$

$-0.970$

$-0.900$

$-0.650$

$-0.0780$

$-0.00970$

0.0280

0.710

0.950

$-3.090$

$-3.000$

$-2.800$

$-2.260$

$-0.960$

$-0.590$

$-0.300$

$-0.510$

$1.800 \quad 95$

$-0.830$

8.020
$8.8 e-09$

$9.5 e-09$

9. $6 e-09$

4. 3e-09

4. $6 e-09$

4. $2 e-10$

$4.1 e-10$

$1.8 e-09$

4. $1 e-09$

$1.8 e-08$

2. $4 \mathrm{e}-06$

$6 e-07$

6. $6 \mathrm{e}-12$

1. $6 e-08$

3. $4 \mathrm{e}-16$

1. $5 e-15$

6. $6 e-16$

1. $9 e-16$

8. $9 \mathrm{e}-17$

$7.1 e-14$

2. $6 e-06$

3. $3 e-16$

$7 e-15$

1. $8 e-10$

5. $2 \mathrm{e}-10$

3. $9 e-17$

2. $6 e-09$

2. $3 e-07$

2. $4 \mathrm{e}-11$

3. $5 e-11$

2. $1 \mathrm{e}-11$

2. $7 e-11$

5. $6 e-11$

2. $4 \mathrm{e}-09$

5. $5 e-09$

$1.1 e-06$

3. $3 e-10$
U R. VGTWETEAWCK.L

U R.VGTWETEAWCK. L

U R.VGTWETEAWCK. L

U R.VGTWETEAWCK. L

U R.VGTWETEAWCK. L

U R.VGTWETEAWCK. L

U R.VGTWETEAWCK. L

U R.VGTWETEAWCK. L

U R.VGTWETEAWCK. L

U R. YENGAWK. T

U K. TIATAQGYAATGQNLNR.T

U K. TIATAQGYAATGQNLNR. T

U K. TIATAQGYAATGQNLNR. T

U K. TIATAQGYAATGQNLNR. T

U K. TIATAQGYAATGQNLNR.T

U K.TIATAQGYAATGQNLNR. T

U K. TIATAQGYAATGQNLNR.T

U K. TIATAQGYAATGQNLNR. T

U K.TIATAQGYAATGQNLNR.T

U K. TIATAQGYAATGQNLNR. T

U K.TIATAQGYAATGQNLNR. T

U K. TIATAQGYAATGQNLNR. T

U K.TIATAQGYAATGQNLNR. T

U K. TIATAQGYAATGQNLNR. T

U K.TIATAQGYAATGQNLNR.T

U K. TIATAQGYAATGQNLNR. T

U K.TIATAQGYAATGQNLNR.T

U R. TFSNISNVMEKPMR.V

U R.TFSNISNVMEKPMR.V

U R. TFSNISNVMEKPMR.V

U R. TFSNISNVMEKPMR.V

U R. TFSNISNVMEKPMR.V

U R.TFSNISNVMEKPMR.V

U R. TFSNISNVMEKPMR.V

U R.TFSNISNVMEKPMR. V + Oxidation (M)

U R.TFSNISNVMEKPMR.V + Oxidation (M)

U K.VDLYANSSYSDYVQTVYTK.Q

U K.VDLYANSSYSDYVQTVYTK.Q 


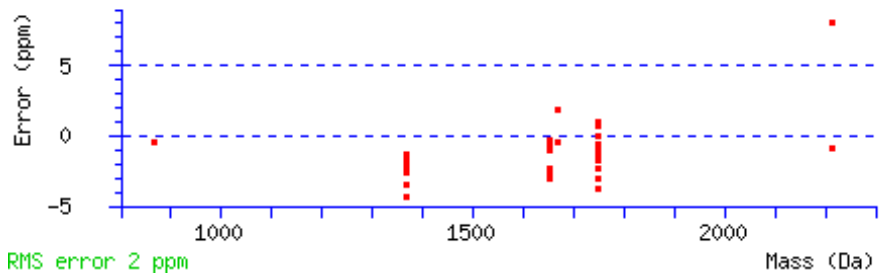

Mascot: http://www.matrixscience.com/

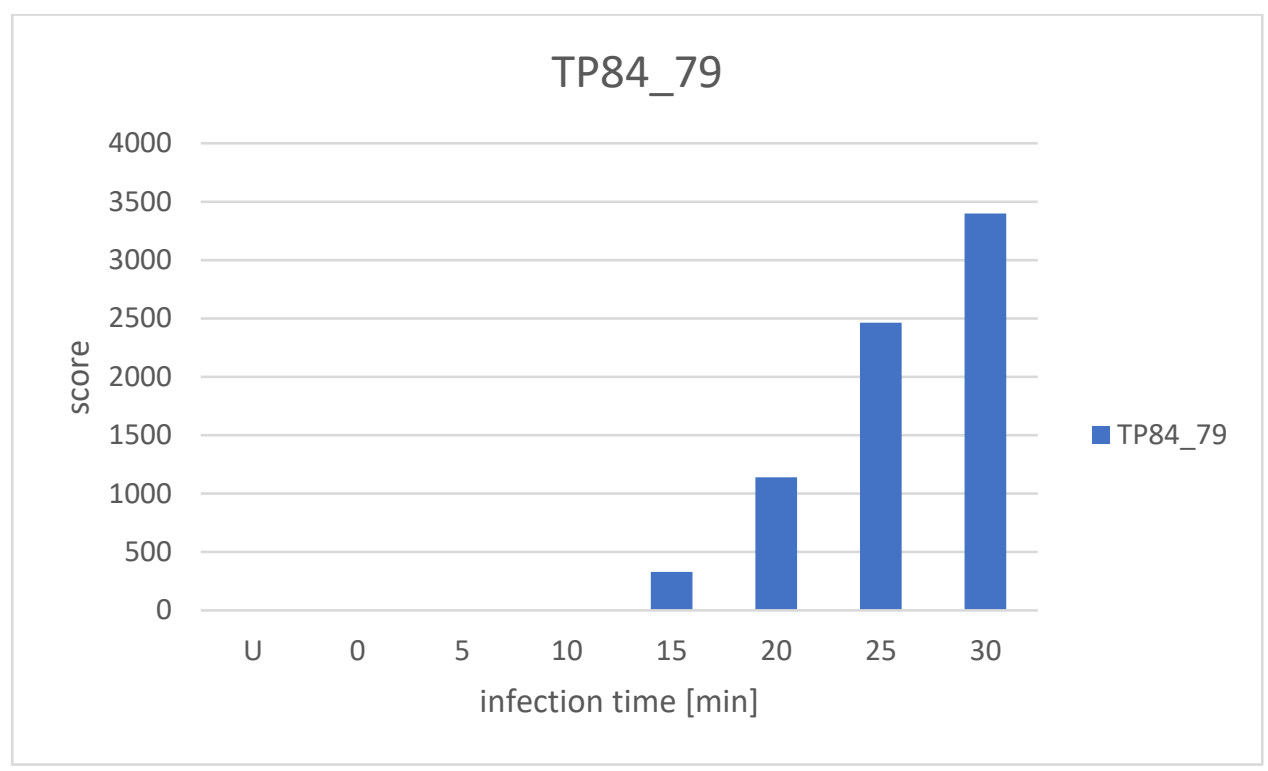




\section{MASCOT Search Results}

\section{Protein View: TP84_80}

$\begin{array}{ll}\text { Database: } & \text { TP84 } \\ \text { Score: } & 166 \\ \text { Nominal mass (Mr): } & 13948 \\ \text { Calculated pI: } & 6.82\end{array}$

Sequence similarity is available as an NCBI BLAST search of 53. against nr.

\section{Search parameters}

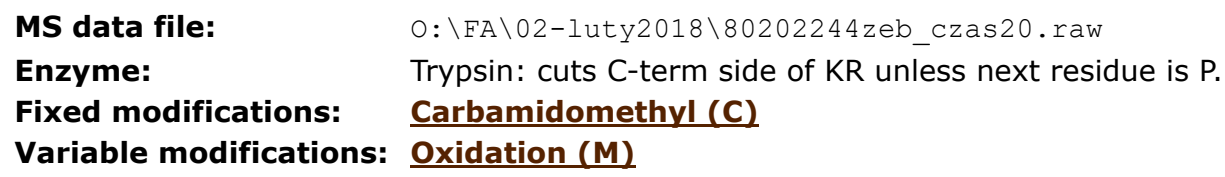

\section{Protein sequence coverage: $9 \%$}

Matched peptides shown in bold red.
1 MGDMATTSKA IELNDNFTYG GTGYLNTSED YMNVYATWET PYNTYVYASM
51 TLQRYEDGVW KNIETKGAYA YYTHQERKHA NVQFTNIAKK GTPMRVKLNL
101 HDGNNPNSPS MQTAYSYSWT R

Unformatted sequence string: 121 residues (for pasting into other applications).
Sort peptides by
- Residue Number
Increasing Mass
Decreasing Mass

Show predicted peptides also

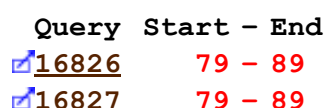

$\checkmark 16827$

$79-89$ $\begin{array}{ccc}\text { Observed } & \text { Mr (expt) } & \text { Mr (calc) } \\ 621.8364 & 1241.6583 & 1241.6517 \\ 621.8366 & 1241.6586 & 1241.6517\end{array}$

$\begin{array}{lcc}\text { ppm } & \text { M } & \text { Score } \\ 5.32 & 0 & 92 \\ 5.60 & 0 & 86\end{array}$

$5.600 \quad 86$
Expect Rank

6. $2 \mathrm{e}-101$

2. $3 e-091$
U Peptide

U K. HANVQFTNIAK.K

U K. HANVQFTNIAK.K

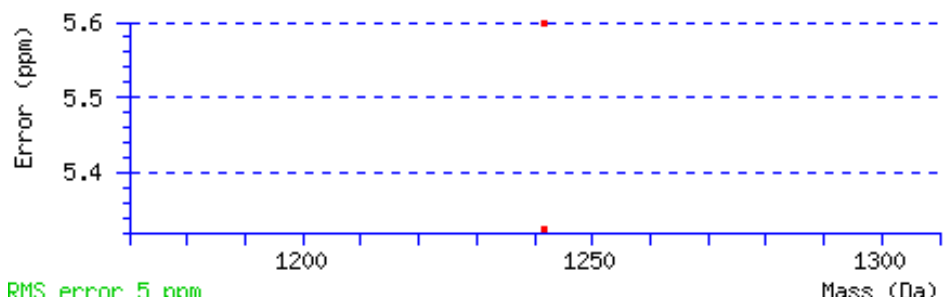

Mascot: http://www.matrixscience.com/ 


\title{
MASCOT Search Results
}

\section{Protein View: TP84_80}

\author{
Database: TP84 \\ Score: $\quad 511$ \\ Nominal mass $\left(\mathbf{M}_{\mathbf{r}}\right): 13948$ \\ Calculated pI: $\quad 6.82$
}

Sequence similarity is available as an NCBI BLAST search of 53. against nr.

\section{Search parameters}

\author{
MS data file: $\quad 0: \backslash F A \backslash 02-1 u t y 2018 \backslash 80202245 z e b \_c z a s 25$.raw \\ Enzyme: $\quad$ Trypsin: cuts C-term side of KR unless next residue is $P$. \\ Fixed modifications: Carbamidomethyl (C) \\ Variable modifications: Oxidation (M)
}

\section{Protein sequence coverage: $\mathbf{3 8 \%}$}

Matched peptides shown in bold red.

1 MGDMATTSKA IELNDNFTYG GTGYLNTSED YMNVYATWET PYNTYVYASM

51 TLQRYEDGVW KNIETKGAYA YYTHQERKHA NVQFTNIAKK GTPMRVKLNL 101 HDGNNPNSPS MQTAYSYSWT R

Unformatted sequence string: 121 residues (for pasting into other applications).

Sort peptides by - Residue Number $\bigcirc$ Increasing Mass $\bigcirc$ Decreasing Mass

Show predicted peptides also

\begin{tabular}{|c|c|}
\hline Query & Start - End \\
\hline 13924 & $67-77$ \\
\hline$\checkmark 13928$ & $67-77$ \\
\hline$\nabla^{\prime 13929}$ & $67-77$ \\
\hline$\checkmark 13930$ & $67-77$ \\
\hline$\widetilde{14377}$ & $78-89$ \\
\hline$\widetilde{14378}$ & $78-89$ \\
\hline$\checkmark 14379$ & $78-89$ \\
\hline $\mathbb{\prime}^{\prime 10813}$ & $79-89$ \\
\hline$\checkmark 35225$ & $98-121$ \\
\hline
\end{tabular}

$\begin{array}{rrr}\text { Observed } & \text { Mr (expt) } & \text { Mr (calc) } \\ 453.5421 & 1357.6043 & 1357.6051 \\ 453.5424 & 1357.6053 & 1357.6051 \\ 453.5426 & 1357.6059 & 1357.6051 \\ 679.8106 & 1357.6067 & 1357.6051 \\ 457.5896 & 1369.7470 & 1369.7466 \\ 457.5896 & 1369.7471 & 1369.7466 \\ 457.5897 & 1369.7473 & 1369.7466 \\ 621.8331 & 1241.6516 & 1241.6517 \\ 918.4150 & 2752.2233 & 2752.2299\end{array}$

\begin{tabular}{rrr}
\multicolumn{1}{c}{ ppm } & M & Score \\
-0.56 & 0 & 55 \\
0.14 & 0 & 57 \\
0.59 & 0 & 59 \\
1.18 & 0 & 97 \\
0.28 & 1 & 59 \\
0.33 & 1 & 52 \\
0.48 & 1 & 55 \\
0.023 & 0 & 98 \\
-2.42 & 0 & 83
\end{tabular}

Expect Rank

3. $2 e-06 \quad 1$

U Peptide

2. $1 \mathrm{e}-06$

U K. GAYAYYTHQER. K

1. $3 e-06$

K GAYAYYTHOER. $K$

$1.2 e-06 \quad 1$ U R.KHANVQFTNIAK.K

$5.6 e-06 \quad 1$ U R.KHANVQFTNIAK.K

3. $3 e-06 \quad 1$ U R.KHANVQFTNIAK.K

$\checkmark \underline{35225} \quad 98-121$

$918.4150 \quad 2752.2233 \quad 2752.2299$

$-2.420 \quad 83$

1. $5 e-10 \quad 1$

U K. HANVQFTNIAK.K

4.9e-09 1 U K. LNLHDGNNPNSPSMOTAYSYSWTR. -

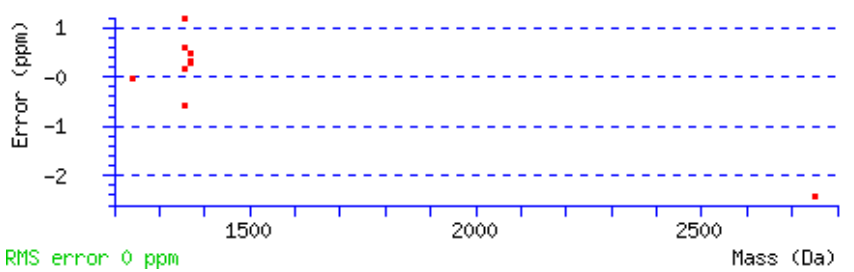

Mascot: http://www.matrixscience.com/ 


\title{
MASCOT Search Results
}

\section{Protein View: TP84_80}

$\begin{array}{ll}\text { Database: } & \text { TP84 } \\ \text { Score: } & 1229 \\ \text { Nominal mass (Mr): } & 13948 \\ \text { Calculated pI: } & 6.82\end{array}$

Sequence similarity is available as an NCBI BLAST search of 53. against nr.

\section{Search parameters}

\author{
MS data file: \\ Enzyme: \\ $0: \backslash F A \backslash 02$-luty2018\80202246zeb_czas30.raw \\ Fixed modifications: \\ Trypsin: cuts C-term side of KR unless next residue is $P$. \\ Variable modifications: Oxidation (M)
}

\section{Protein sequence coverage: $19 \%$}

Matched peptides shown in bold red.

1 MGDMATTSKA IELNDNFTYG GTGYLNTSED YMNVYATWET PYNTYVYASM

51 TLQRYEDGVW KNIETKGAYA YYTHQERKHA NVQFTNIAKK GTPMRVKLNL

101 HDGNNPNSPS MQTAYSYSWT R

Unformatted sequence string: 121 residues (for pasting into other applications).

Sort peptides by $\bullet$ Residue Number $\bigcirc$ Increasing Mass $\bigcirc$ Decreasing Mass

Show predicted peptides also

\begin{tabular}{|c|c|}
\hline Query & Start - End \\
\hline$\nabla^{\prime 19644}$ & $67-77$ \\
\hline 19645 & $67-77$ \\
\hline$\sigma^{\prime} 19646$ & $67-77$ \\
\hline$\nabla^{\prime} 19647$ & $67-77$ \\
\hline$\square^{\prime} 19648$ & $67-77$ \\
\hline$\sigma^{\prime} 19649$ & $67-77$ \\
\hline$\sigma^{\prime 19650}$ & $67-77$ \\
\hline$\sigma^{19651}$ & $67-77$ \\
\hline$\square^{\prime 19652}$ & $67-77$ \\
\hline $\mathbb{D}^{\prime 19653}$ & $67-77$ \\
\hline$\square^{20224}$ & $78-89$ \\
\hline$\sigma^{\prime 15148}$ & $79-89$ \\
\hline$\sigma^{\prime 15149}$ & $79-89$ \\
\hline$\varpi^{\prime} 15150$ & $79-89$ \\
\hline$\square^{\prime 15152}$ & $79-89$ \\
\hline
\end{tabular}

$\begin{array}{rrr}\text { Observed } & \text { Mr (expt) } & \text { Mr (calc) } \\ 679.8091 & 1357.6036 & 1357.6051 \\ 679.8093 & 1357.6041 & 1357.6051 \\ 679.8094 & 1357.6043 & 1357.6051 \\ 453.5421 & 1357.6044 & 1357.6051 \\ 679.8095 & 1357.6045 & 1357.6051 \\ 453.5421 & 1357.6045 & 1357.6051 \\ 453.5422 & 1357.6049 & 1357.6051 \\ 453.5423 & 1357.6051 & 1357.6051 \\ 679.8099 & 1357.6052 & 1357.6051 \\ 453.5425 & 1357.6056 & 1357.6051 \\ 685.8818 & 1369.7490 & 1369.7466 \\ 621.8331 & 1241.6517 & 1241.6517 \\ 621.8332 & 1241.6518 & 1241.6517 \\ 621.8332 & 1241.6519 & 1241.6517 \\ 621.8338 & 1241.6530 & 1241.6517\end{array}$

$\begin{array}{rrr}\text { ppm } & \text { M } & \text { Score } \\ -1.07 & 0 & 103 \\ -0.75 & 0 & 97 \\ -0.56 & 0 & 103 \\ -0.54 & 0 & 69 \\ -0.43 & 0 & 103 \\ -0.41 & 0 & 90 \\ -0.14 & 0 & 90 \\ 0.033 & 0 & 90 \\ 0.060 & 0 & 97 \\ 0.39 & 0 & 90 \\ 1.73 & 1 & 88 \\ 0.041 & 0 & 101 \\ 0.15 & 0 & 98 \\ 0.19 & 0 & 96 \\ 1.06 & 0 & 96\end{array}$

Expect Rank U Peptide

5.2e-11 1 U K.GAYAYYTHQER.K

$1.9 e-10 \quad 1 \quad$ U K.GAYAYYTHQER.K

$5.2 e-11 \quad 1$ U K.GAYAYYTHQER.K

$1.3 e-07 \quad 1 \quad$ U K.GAYAYYTHQER.K

5.3e-11 1 U K.GAYAYYTHQER.K

$9.5 e-10 \quad 1$ U K.GAYAYYTHQER.K

9.4e-10 1 U K.GAYAYYTHQER.K

$9.4 \mathrm{e}-10 \quad 1$ U K.GAYAYYTHQER.K

$1.8 e-10 \quad 1$ U K.GAYAYYTHQER.K

$9.5 e-10 \quad 1$ U K.GAYAYYTHQER.K

$1.7 e-09 \quad 1$ U R.KHANVQFTNIAK.K

$8.8 e-11 \quad 1$ U K. HANVQFTNIAK.K

$1.6 e-10 \quad 1$ U K.HANVQFTNIAK.K

$2.8 e-10 \quad 1 \quad$ U $K$. HANVQFTNIAK.K

2.3e-10 1 U K. HANVQFTNIAK.K

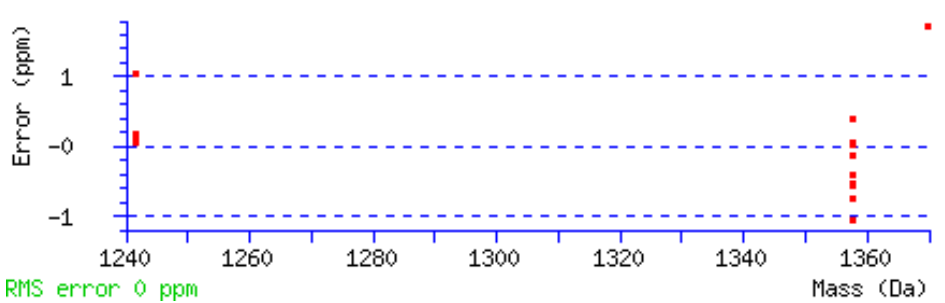

Mascot: http://www.matrixscience.com/ 
TP84_80

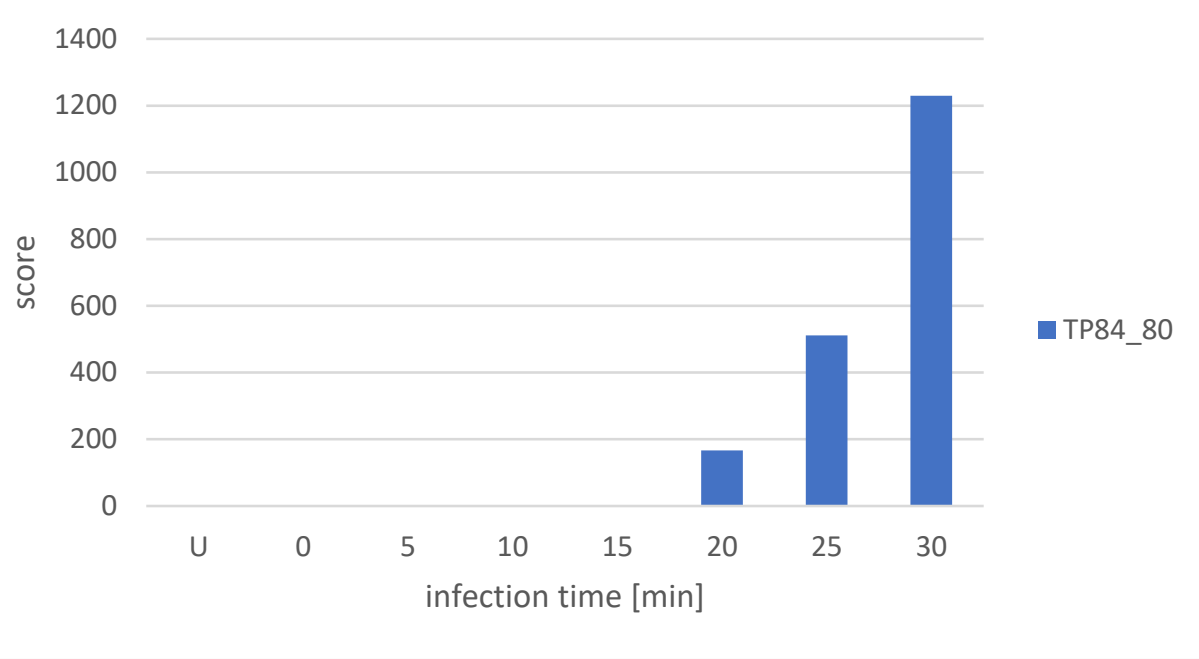

